\title{
A Ground Water Flow Model for the A/M Area of the SRS (U)
}

C. M. Beaudoin, Schreuder \& Davis, Inc.

P. J. Schrouder, Schreuder \& Davis, Inc.

J. S. Haselow, Savannah River Laboratory

June, 1991

Authentication: \&.d Taselore

Approved By:

D. B. Moore, Section Manager

Environmental Sciences Section

Savannahi River Laboratory

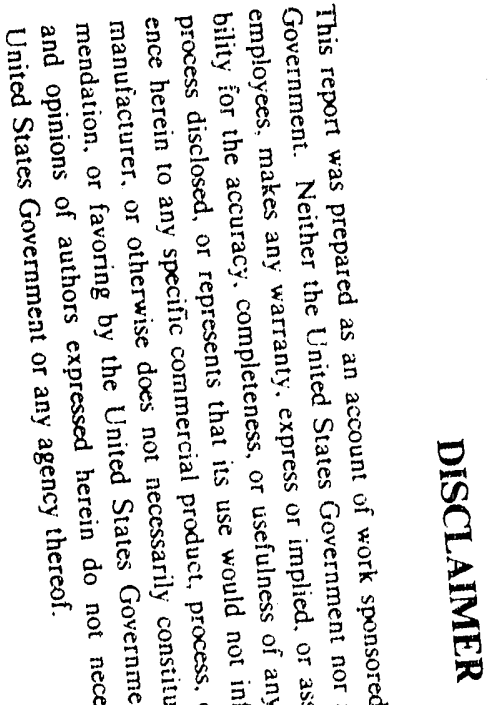

WESTINGHOUSE SAVANNAH RIVER COMPANY

SAVANNAH RIVER LABORATORY

SAVANNAH RIVER SITE

AIKEN, SC 29808

Propared for the U.S. Department of Energy under Contract No. DE.ACO9-89SR18035 


\section{TABLE OF CONTENTS}

EXECUTIVE SUMMARY $\ldots \ldots \ldots \ldots \ldots \ldots \ldots \ldots \ldots \ldots \ldots \ldots \ldots$ iv

1.0 INTRODUCTION $\ldots \ldots \ldots \ldots \ldots \ldots \ldots \ldots \ldots \ldots \ldots \ldots \ldots \ldots \ldots \ldots$

1.1 PURPOSE AND OBJECTIVES $\ldots \ldots \ldots \ldots \ldots \ldots \ldots \ldots \ldots$

1.2 DESCRIPTION OF MODELING SITE $\ldots \ldots \ldots \ldots \ldots \ldots \ldots \ldots$

1.3 HISTORICAL DEVELOPMENT OF THE MODEL .......... 2

2.0 HYDROGEOLOGICAL SETTING $\ldots \ldots \ldots \ldots \ldots \ldots \ldots \ldots \ldots \ldots \ldots \ldots$

2.1 GENERAL GEOLOGY $\ldots \ldots \ldots \ldots \ldots \ldots \ldots \ldots \ldots \ldots \ldots$

2.2 GROUNDWATER FLOW $\ldots \ldots \ldots \ldots \ldots \ldots \ldots \ldots \ldots \ldots \ldots \ldots$

2.3 SURFACE WATER FLOW $\ldots \ldots \ldots \ldots \ldots \ldots \ldots \ldots \ldots \ldots \ldots$

2.4 RAINFALL RECHARCE $\ldots \ldots \ldots \ldots \ldots \ldots \ldots \ldots \ldots \ldots$

3.0 FIELD DATA COMPILATION AND ANALYSIS $\ldots \ldots \ldots \ldots \ldots \ldots \ldots$



3.2 STREAM FLOW DATA $\ldots \ldots \ldots \ldots \ldots \ldots \ldots \ldots \ldots \ldots \ldots$

3.3 WATER LEVEL DATA $\ldots \ldots \ldots \ldots \ldots \ldots \ldots \ldots \ldots$

3.4 AQUIFER TEST DATA $\ldots \ldots \ldots \ldots \ldots \ldots \ldots \ldots \ldots \ldots \ldots \ldots$

3.6 WATER QUALITY DATA $\ldots \ldots \ldots \ldots \ldots \ldots \ldots \ldots \ldots \ldots \ldots \ldots$

4.0 GROUNDWATER MODEL SET-UP $\ldots \ldots \ldots \ldots \ldots \ldots \ldots \ldots \ldots \ldots \ldots$

4.1 SELECTION OF MODEL CODE ................. 13

4.2 MODEL SIMULATION OF HYDROGEOLOGIC SETTING . . . . 13

4.2.1 Boundary Conditions ................... 14

4.2.2 Aquifer Parameters ..................... 15

4.2.3 Recharge ......................... 17

4.3 MODEL SIMULATION OF GROUNDWATER WITHDRAWALS .. 17

5.0 MODEL CALIBRATION $\ldots \ldots \ldots \ldots \ldots \ldots \ldots \ldots \ldots \ldots \ldots \ldots \ldots \ldots$

5.1 DESCRIPTION OF CALIBRATION PROCESS .......... 18

5.2 CALIBRATION TO OBSERVED WATER LEVELS ......... 19

5.2.1 Predicted Water Levels, Water Table ............. 19

5.2.2 Predicted Water Levels, Upper Congaree ........... 20

5.2.3 Predicted Water Levels, Lower Congaree ........... 20

5.2.4 Predicted Water Levels, Black Creek ............ 20

5.3 CALIBRATION TO SURFACE WATER FLOW .......... 21

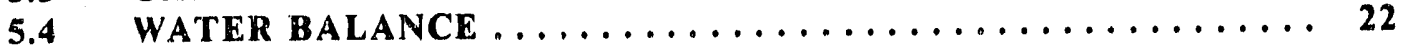

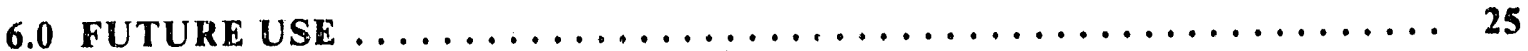

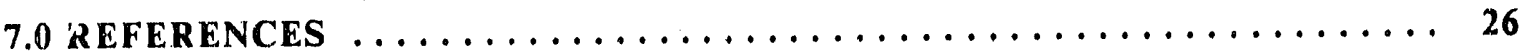

A Ground Water Flow Model for the A/M Area of the SRS (U) Page it 


\section{LIST OF FIGURES}

Figure 1. Location of the Savannah River Site and Modeled Area. ....... 28

Figure 2. Model Area and Nodal Grid. ..................... 29

Figure 3. Location of Recovery Wells and Production Wells. ......... 30

Figure 4. Topography, Watershed Boundary and Surface Water Features. . . 31

Figure 5. Lithology and Relationship Between Hydrostratigraphic and

Stratigraphic Nomenclature. ................. 32

Figure 6. Hydrostratigraphic Cross-Section and Model Layer Definitions. . 33

Figure 7. Observed Water Levels in the Water Table. ............. 34

Figure 8. Observed Water Levels in the Upper Congaree. ............ 35

Figure 9. Observed Water Levels in the Lower Congaree. ........... 36

Figure 10. Observed Water Levels in the Black Creek. ............. 37

Figure 11. Observed TCE Concentration in Water Table............ 38

Figure 12. Observed TCE Concentration in Upper Congaree........... 39

Figure 13. Observed TCE Concentration in the Lower Congaree......... 40

Figure 14. Observed TCE Concentration in the Black Creek.......... 41

Figure 15. Boundary Conditions and Transmissivity in Model Layer 1. . . . 42

Figure 16. Boundary Conditions and Transmissivity in Model Layer 2. ..... 43

Figure 17. Boundary Conditions and Transmissivity in Model Layer 3. ..... 44

Figure 18. Boundary Conditions and Transmissivity in Model Layer 4..... 45

Figure 19. Calibrated Leakance Map for Model Layer 1. ........... 46

Figure 20. Calibrated Leakance Map for Model Layer 2. ............. 47

Figure 21. Calibrated Leakance Map for Model Layer 3. ............. 48

Figure 22. Modeled Rainfall Recharge. .................... 49

Figure 23. Observed and Model-Predicted Water Levels in Layer 1........ 50

Figure 24. Observed and Model-Predicted Water Levels in Layer 2....... 51

Figure 25. Observed and Model-Predicted Water Levels in Layer 3........ 52

Figure 26. Observed and Model-Predicted Water Levels in Iayer 4........ 53

Table 1. Volumetric Budget for Entire Model at End of Time Step 1 in Stress Period 1.......................... 22

Table 2. Volumetric Flow Budget for Each Model Layer for Time Step 1 in

Stress Period 1. .......................... 24

\section{LIST OF APPENDICES}

Appendix A. Water Level Data

Appendix B. An Alternetive Interpretation of the TCE Data in the Southern Sector of the Lower Congaree

Appendix C. Hardcopy of Model Input

Appendix D. Location and Pumpage Rates of Production and Recovery Wells Used in Groundwater Model

Appendix E. Description of Calibration Process

A Ground Water Flow Model for the A/M Area of the SRS (U) Page iii 


\section{EXECUTIVE SUMMARY}

In 1984 and 1985 , a quasi three-dimensional groundwater flow model was developed for the A/M Area (Savannah River Laboratory Complex) of the Savannah River Site to assist in the design of a groundwater recovery well network to remediate groundwater contaminated with chlorinated hydrocarbon solvents. In 1986, the existing groundwater flow model was used to evaluate the effectiveness of groundwater remediation systems. Since the original model was developed, additional monitoring wells have been installed, the understanding of the hydrogeologic system has improved and the horizontal and vertical extent of the contamination in the groundwater systems has been better defined.

The objective of this study is to update and improve the existing A/M Area groundwater flow model by incorporating recent hydrologic information. The new model is calibrated to 1989-1990 groundwater levels and surface water flows. The model area is approximately 31 square miles and encompasses a manufacturing and processing area designated the $\mathrm{A} / \mathrm{M}$ Area, a previous waste effluent disposal basin and overflow to a natural depression (Lost Lake), and several surface water features. The simulations are run on a 80386-based personal computer using the public-domain groundwater flow code MODFLOW.

This calibrated coarse-grid model is a base model. Specific areas of interest can be further discritized to provide more detailed and accurate water level elevations for use in particle tracking and capture analysis. The particle tracking and capture analysis are used to evaluate the groundwater contamination capture and removal capacity of proposed recovery wells.

A Ground Water Flow Model for the A/M Area of the SRS (U) 


\subsection{INTRODUCTION}

\subsection{PURPOSE AND OBJECTIVES}

The purpose of this study is to develop a groundwater flow model for the $A / M$ Area within the Savannah River Site (SRS) so that the effectiveness of groundwater remediation systems can be simulated. This report describes the set-up and calibration of a coarse-grid model for the area. The coarse-grid model can be subsequently refined within an area of interest so that a capture zone analysis of contaminated groundwater can be simulated. The objective of this project is to update and improve the existing A/M Area model by incorporating recent hydrologic information.

\subsection{DESCRIPTION OF MODELING SITE}

The Savannah River Site, operated by Westinghouse Savannah River Company (WSRC), is located in South Carolina along the banks of the Savannah River. The location of the SRS and the area modeled by Schreuder \& Davis, Inc. (SDI) are indicated in Figure 1. The model encompasses a manufacturing and processing area called the A/M Area, a previous waste effluent disposal basin and overflow to a natural depression (Lost Lake), and several surface water features. The modeled area is a rectangle $25,000 \mathrm{ft}$ from east to west and $35,000 \mathrm{ft}$ from north to south. SRS plant coordinates from the south west corner of $N 77,000$ and $E 33,500$ ft to the northeast corner of N112,000 and E58,500 ft are included. Plant north is $36^{\circ}$ to the west of true north. The total modeled area is 20,087 acres ( 31.4 square miles). The nodal spacing of the model is $1,000 \times 1,000$ feet $(\mathrm{ft}$ ) spaced on a regular basis (Figure 2). 


\subsection{HISTORICAL DEVELOPMENT OF THE MODEL}

The first quasi three-dimensional ground water flow model for the A/MArea was prepared by S.S. Papadopulos \& Associates (SSP\&A) in 1986 to evaluate the ef fectiveness of the A/M Area recovery well network (Figure 3) and to simulate chemical transport from the M-Area settling basin and Lost Lake (Papadopulos, 1986 and 1987). That model was used to develop a groundwater recovery well network for the SRL Area (Schreuder et al., 1990). Since the original three-dimensional model was developed, additional monitoring wells have been installed and the understanding of the hydrogeologic system has improved. This provides the motivation for updating the original mode!. 


\subsection{HYDROGEOLOGICAL SETTING}

The SRS is located on the Aiken Plateau in the Upper Atlantic Coastal Plain of South Carolina. The unconsolidated and semi-consolidated sediments underlying the model area are part of a Tertiary to late Cretaceous-age wedge that dips and thickens toward the Atlantic Ocean. The land surface is deeply incised by surface water drainage features. The topography in the model area ranges from 380 feet mean sea level (MSL) in the A/M Area to 120 feet MSL along Upper Three Runs Creek (Figure 4).

The major drainage divide in the model area is along the north-south (directions referenced to true north) trending ridge on which the $\mathrm{A} / \mathrm{M}$ Area is located (Figure 4). Surface water on the east side of the ridge drains into Tim's Branch which flows south into Upper Three Runs Creek. Upper Three Runs Creek flows southwest into the Savannan River. Surface water on the west side of the ridge drains into a series of wetlands to the southwest.

Surface water southwest of the A/M Area also drains into a depressional area called Lost Lake. It has been suggested that Lost Lake is a Carolina Bay feature. These features typically form temporary wetlands which fill and drain with the cycles of rainfall. In the past, Lost Lake received overflow from the M-Area settling basin. Lost Lake was dry during the time period used for calibration and is, therefcre, not a source of recharge in the model. 


\subsection{GENERAL GEOLOGY}

Fallaw and Sargent (1986) proposed a detailed description of the general geology. Their stratigraphic descriptions are summarized in Figure 5. The relationship of the hydrostratigraphic units to geologic formations is also shown in Figure 5. These hydrostratigraphic units are, in descending order, the water table, Upper Congaree, Lower Congaree, Ellenton, and Black Creek. A northwest-southeast cross-section of the hydrostratigraphy in the model area and its relationship to the hydrogeologic layers used in the groundwater model is shown in Figure 6 (Aadland and Bledsoe, 1990). Four water-bearing zones in the SRL, area are included in the model.

In the A/M Area, the upper most geologic formation is the Upland Unit followed in descending order by the Tobacco Road, Dry Branch and the McBean. The water table unit is the saturated portion of the McBean Formation which consists of clayey sand and calcareous clayey sand. The top of the water table unit is over $100 \mathrm{fect}$ below land surface. The stratigraphic units above the McBean contain clay and silty sand lenses that may act as local confining or semi-confining layers. The McBean and the underlying Congaree Formation are separated by a clay layer informally referred to as the "Green Clay."

The Congaree consists of sand with clay lenses and is approximately ten times more permeable than the McBean. The Congaree is divided into an upper and lower unit in the A/M Area by a clay layer. This clay layer thins and becomes discontinuous to the south.

The Ellenton consists of dark lignitic clay with some coarse sand units and is separated from the Congaree by an unconformity. The Ellenton is considered to be the principle confining bed that separates the water table and the Congaree from the ciecper 
hydrologic system. However, it is known to thin and disappear in the northern portion of the model area (Aadland and Bledsoc, 1990).

The deepest aquifer system at the SRS is in Cretaceousmage sediments that were previously denoted the Black Creek and Middendorf Formations (c.g., Papadopulos, 1986). This group of sediments has been recently designated Aquifer System I (Aadland and Bledsoe, 1990). This aquifer system produces large quantities of high-quality water. For this modeling effort, only the upper portion of Aquifer System I is considered and is designated as the Black. Creek to remain consistent with previous reports for the $A / M$ Area.

\subsection{GROUNDWATER FLOW}

Groundwater in the Upper Congaree and Lower Congaree generally flows south to southeast towards Upper Three Runs Creek. Groundwater flow along the northwest side of the model area is southwest towards a low wetland area that drains into the Savannah River approximately three miles to the southwest. Groundwater in the Black Creek flows south towards the Savannah River (Christensen \& Gordon, 1983). In the SRS area, the Ellenton is an aquitard so the groundwater flow in this unit is nearly vertical. Vertical flow in the Ellenton is downward in the $\mathrm{A} / \mathrm{M}$ Area where hydraulic heads in the Black Creek are less than in the hydrostratigraphic unit above the Ellenton. Vertical flow is upward in the Ellenton around Upper Three Runs Creck where hydraulic heads in the Black Creek are greater than those in the unit above the Ellenton. 


\subsection{SURFACE WATER FLOW}

The deeply incised streams in the region dissect the McBean Formation and divide the hydrogeologic unit into separated subunits, each having its own recharge and discharge area. The regional hydrostratigraphic cross-section in Figure 6 (hereafter referred to as the regional cross-section) shows Tim's Branch and Upper Three Runs Creek to be incised into the Upper Congaree. The Upper Congaree also crops out in the southwest wetlands. Groundwater in the water table and Upper and Lower Congaree discharge into the streams and wetlands.

\subsection{RAINFALL RECHARGE}

The average annual rainfall at the SRS is approximately 47 inches per year (in/yr). Studies by the U.S. Geological Survey (Cahill, 1982) and Savannah River Laboratories (Hubbard and Emslie, 1984 and Hubbard, 1986) indicate the average annual recharge rate is approximately $15 \mathrm{in} / \mathrm{yr}$. Recharge rates may vary in areas of steep topography. 


\subsection{FIELD DATA COMPILATION AND ANALYSIS}

Synchronous water level data were provided by SRL to calibrate this model. In accordance with SDI's quality assurance/quality control plan, all data were checked during compilation and analysis and discrepancies were noted to SRL.in writing.

\subsection{RAINFALL DATA}

Daily rainfall data from a rain gauge in the A/M Area for 1967 to 1987 were provided by SRL. Rainfall in the A/M Area for the years 1967 through 1987 ranged from 37 to 68 inches (in) and averaged $47 \mathrm{in} / \mathrm{yr}$.

\subsection{STREAM FLOW DATA}

Stream flow data were used to estimate the magnitude of surface water recharge or discharge to/from Tim's Branch and Upper Three Runs Creck to the model. Strcam flow data from U.S.G.S. gauging stations on Tim's Branch at Road C and Upper Threc Runs Creck at Road C and Road A, for water year (WY) 1987, were provided by SRL (U.S.G.S., 1987). The locations of the gauging stations on Tim's Branch at Road C and Upper Three Runs Creek at Road C are shown on Figure 4. The gauging station on Upper Threc Runs Creck at Road $A$ is just west of the model area. Daily stream flow data for Upper Three Runs Creck for WY 1974 thiough 1988 were provided on floppy disk. The drainage area for Tim's Branch is 17.5 square miles $\left(\mathrm{mi}^{2}\right)$ of which the model area is estimated to encompass the lower $7.6 \mathrm{mi}^{2}(43 \%)$. The mean discharge for WY 1987 was 6.71 cubic fect per second (cfs). The average base flow is estimated to range 
from 3 to $5 \mathrm{cfs}$. Theoretically, approximately 1.3 to $2.2 \mathrm{cfs}$ of the base flow should be contributed by the model area. However, Lockwood Grecne Engincers (1983) indicate that flow in the upper portion of Tim's Branch is seasonal. Therefore, a larger percentage of the base flow may be contributed by the modeled arca.

The elcuation of the stream bottom in the upper portion of Tim's Branch, northeast of the A/M Area, is above the water table. Thus, this part of Tim's Branch is a losing strcam. The stream is incised into the water table southeast of the A/M Area and base flow is iustained by discharge from the water table. Base flow in the lower portion of the stream is sustained by discharge from the Upper Congaree.

The discharge through the gauging station at Road $C$ and Upper Three Runs Creek docs not include discharge from the entire model area. Data from the gauging station at Road $A$ and Upper Three Runs Creck were used for calibration. The total drainage area for Upper Three Runs Creck above Road A is $203 \mathrm{mi}^{2}$. Upper Three Runs Creck and koad $\mathrm{A}$ intersect 1.25 miles west of the model area. Approximately twothirc's of the model area cast of the drainage divide makes up approximately $10 \%$ of the Upper Threc Runs Creek drainage area above Road A. The mean discharge at the Road A gauging station for WY 1987 was $239 \mathrm{cfs}$. The average base flow is estimated to range from 120 to $200 \mathrm{cfs}$. Thus the base flow contribution from the model area to Upper three Runs Creck is estimated to be 12 to $20 \mathrm{cfs}$. Base flow in Upper Three Runs Creek within the model area is sustained by the discharge of groundwater from the Upper and Lower Congaree. 


\subsection{WATER LEVEL DATA}

As part of the groundwater remediation program, an extensive monitoring well network in the A/M Area is used to measure groundwater levels and to collect samples for analysis to determine groundwater quality on a quarterly basis. Data from the last three quarters of 1989 and the first quarter of 1990 were provided to SDI to use as model calibration data. Water level data for the water table, Upper Congaree, Lower Congaree and Black Creek were averaged and contoured to produce the average observed water level maps in Figures 7 through 10. The list of the wells used in contouring these maps is provided in Appendix A. For the purpose of clarity, not all data points are posted on the maps. The data is concentrated in the northeast portion of the model. Topography and surface water elevations were used as a guide to approximate the groundwater level contours in areas of insufficient data (Department of Encrgy, 1989). Water levels southeast of Upper Three Runs Creck arc from Duffield, et al., 1989.

As part of SDI's quality assurance plan, the water level data were checked during analysis. A list of wells with questionable data was compiled and sent to SRL (SDI, 1990a). A number of wells were not used for reasons such as: improper well construction, abandoned or dry wells, or perched water table. A list of the wells not used in contouring the observed water level maps is provided in Appendix $A$ as Table A-5. The observed water level maps for each layer were approved by SRL for calibration of this model. 


\subsection{AQUIFER TEST DATA}

Aquifer pumping tests were performed by Geraghty \& Miller (1985) on recovery wells RW-3 through RW-11 in the central A/M Area. Unfortunately, these tests provide little information on specific hydrostratigraphic units because the punping wells are screencd across the water table, Upper Congaree and Lower Congaree. Many of the monitoring wells, used as observation wells during these tests, are only screened in one layer. However, in several of the recovery well pumping tests, other recovery wells (also screened in water table and Upper and Lower Congaree) were used as observation wells. The total transmissivity for the water table and Upper and Lower Congaree calculated for these wells ranged from 2,390 feet squared per day $\left(\mathrm{ft}^{2} /\right.$ day) to $5,940 \mathrm{ft}^{2} /$ day (Papadopulos, 1986).

Values of hydraulic conductivity used in this study were selected from previous models and are assumed to be reasonable numbers for the types of sedimentary deposits found in the water bearing zones. Unit thicknesses used are from the regional cross. section (Figure 6). A specific capacity of about 4 gallons per minute per foot $(\mathrm{gpm} / \mathrm{ft}$ ) of drawdown recently measured in recovery well RW-16 (Figure 3) suggests there is an area of lower transmissivity south of the A/M Area (southern sector). RW-16 is screened in the Upper and Lower Congaree. Using Walton's (1970) method, this specific capacity is equivalent to a transmissivity of $1,340 \mathrm{ft}^{2} /$ day. This lower transmissivity is corsistent with data by Fallaw (1990) which indicates a depression or trough in the Upper and Lower Congaree south of the A/M Area.

An aquifer pumping test was performed on production well PW-20A which is screened in both the Black Creek and the Middendorf (Geraghty \& Miller, i985). A summary of the aquifer test data in Papadopulos, 1986, shows the total transmissivity A Ground Water Flow Model for the A/M Area of the SRS (U) Page 10 
to range from 10,400 to $14,200 \mathrm{ft}^{2} /$ day. The hydraulic conductivity in the Black Creek is estimated as half of the hydraulic conductivity of the underlying hydrologic unit. Using this hydraulic conductivity ratio and the thicknesses from the regional crosssection, the range in transmissivity for the Black Creek in the A/M Area is 3,185 to $5,060 \mathrm{ft}^{2} /$ day.

\subsection{AQUIFER AND AQUITARD THICKNESS DATA}

The sources of the aquifer thickness data used in this coarse grid model are the regional hydrostratigraphic cross-section (Aadland and Bledsoe, 1990; Figure 6) and data from Fallaw, 1990. The cross-section has a north-south orientation through the model arca. The Fallaw data is confined to the A/M Area. The source of the aquitard data used in this calibration is data from Fallaw and Sargent (1986), and from the 1987 Papadopulos model report.

\subsection{WATER QUALITY DATA}

Water quality data and contour maps of the trichloroethylene (TCE) concentrations in the water table, Upper Congarec, Lower Congaree and Black Creek from fourth quarır, 1990 (4Q90), were provided by SRL (WSRC, 1990). The water quality data are in parts per billion (ppb). The water quality data will be used in the zone of capture modeling. The contours are shown in Figures 11 through 14. As part of SDI's QA/QC plan, data points were checked and discrepancies were noted in writing to SRL (SDI, 1990b).

An alternative interpretation of the same TCE concentration data for the Lower Congaree in the southern sector was provided by SDI for SRL's consideration as shown 
in Appendix B. These contours were generated using the $4 \mathrm{Q} 90$ data points and the direction of groundwater flow indicated by the observed average water level contour maps as a guide (SDI, 1991). 


\subsection{GROUNDWATER MODEL SET-UP}

\subsection{SELECTION OF MODEL CODE}

The public-domain groundwater flow code MODFLOW was chosen for this model. The code is widely used and accepted by regulatory agencies. MODFLOW operates on 80386-based personal computers (PC), and MODFLOW files can be easily used with a number of particle tracking programs.

The version of MODFLOW used in this study is run by a FORTRAN compiler (FTN77) developed at the University of Salford, England to allow for the use of extended and virtual memory. The code was modified to provide the data input files for the particle tracking program. These changes are documented in the report by Schreuder et al., 1990. Copies of the codes with changes have been provided to SRL on floppy disk.

The MODFLOW input files for this calibrated model are provided both on disk and hard copy (Appendix C) in this report.

\subsection{MODEL SIMULATION OF HYDROGEOLOGIC SETTING}

The $25,000-\mathrm{ft}$ by 35,000 - $\mathrm{ft}$ area in this study has the same dimensions as in the previous SSP\&A and SDI models. Four hydrogeological layers are simulated and an evenly-spaced coarse grid of $1,000 \mathrm{ft} \times 1,000 \mathrm{ft}$ cells is used over the entire area (Figure 2). Each layer is made up of 25 by 35 cells (875) for a total of 3,500 cells in the model. A model-simulated hydrogeological layer consists of an aquifer and underlying aquitard. The layers are in descending order; the water table, Upper Congarec, Lower 
Congaree and Black Creck. In previous models, the Ellenton was modeled as a separate layer. In this simulation, the Ellenton is modeled as the aquitard underlying the Lower Congaree in layer 3. The model layers and their relationship to the stratigraphy are shown on Figure 6.

\subsubsection{Boundary Conditions}

The water table unit is only present at the higher elevations in the northeast pertion of the model and is therefore not simulated over the entire area. The areal extent of the water table was determined by comparing water levels between the Upper Congaree and the units above. Based on the regional cross-section in Figure 6, the Upper Congaree, Lower Congaree and Black Creek are simulated over the entire model area. Constant-head boundary conditions are used in all layers where the observed water level contours indicate flow into or out of the modeled area. The discharge of groundwater to streams and wetlands is simulated in this model by nodes with headdependent discharge (general-head boundary nodes).

To simulate ground water flow in the water table, constant-head nodes are placed on the northern, western, and part of the southern boundaries of layer 1 (Figure 15). A report by Lockwood Greene Engineers, Inc. (1983) on the stream flow of Tim's Branch indicates that the upper portion of Tim's Branch has only seasonal flow. Because much of the stream in layer 1 is not a constant source of water, that portion is not simulated in the mode! with general-head boundary (GHB) nodes.

To simulate groundwater flow out of model layer 2 (Upper Congaree), constanthead nodes are used in the northeast as shown in Figure 16. The regional cross-section shows Tim's Branch and Upper Three Runs Creck to be incised into the Upper A Ground Water Flow Model for the A/M Area of the SRS (U)

Page 14 
Congaree. Constant-head nodes are used in the southeast corner of the model to simulate water levels in the Upper and Lower Congaree south of Upper Three Runs Creek (Duffield et al., 1989). This area is included in the model to aid in the surface water flow calibration. GHB nodes are used to simulate both streams and the wetlands to the southwest.

It is likely that some of the groundwater in the Lower Congaree and discharges in to the wetlands. No-flow boundary conditions were used along the soutinwestern edge of layer 3 (Lower Congaree) to induce upward flow into layer 2 and simulate discharge to the wetlands. These boundary conditions result in model-predicted water levels and gradients in layers 2 and 3 that satisfactorily reproduce the observed average water levels. There is insufficient data in this area to estimate the amount of discharge to the wetlands from the Upper and Lower Congaree.

To simulate groundwater flowing out of layer 3 (Lower Congaree), constanthead nodes are used along the northern boundary. As in layer 2, constant heads are also used to simulate groundwater flowing into the model in the southeast corner as shown in Figure 17.

The regional groundwater flow in the Black Creek is to the southwest. Constanthead nodes were used on the northeast, east and west boundaries to simulate the observed regional groundwater flow (Figure 18).

\subsubsection{Aquifer Parameters}

A total transmissivity of $2,805 \mathrm{ft}^{2} /$ day is used for the upper three layers in the northern half of the model. As in the SSP\&A and previous SDI models, layer 1 is modeled as a confined layer with a transmissivity of $175 \mathrm{ft}^{2} /$ day (Figure 15). There is 
insufficient data to accurately model the water table as an unconfined layer, especially along the edges where the unit pinches out. The output from this calibrated model will be used for particle tracking to model the zone of capture for recovery wells. Modeling the water table unit as confined or unconfined makes no difference in the particle track because only the water levels and the hydraulic conductivity are utilized in the program.

The total transmissivity for the Upper and Lower Congaree is $2,630 \mathrm{ft}^{2} / \mathrm{day}$ in the northern half of the model. The distribution of transmissivity in the Upper Congarec is shown in Figure 16. The distribution of transmissivity in the Lower Congarec is shown in Figure 17. A hydraulic conductivity of $44 \mathrm{ft} / \mathrm{day}$ is used for both layers (Papadopulos, 1987). In layer 2, a transmissivity of $1,750 \mathrm{ft}^{2} / \mathrm{day}$ is used in the north and a value of $3,100 \mathrm{ft}^{2} /$ day is used in the south. In layer 3, a transmissivity of $880 \mathrm{ft}^{2} /$ day is used in the north and a value of $2,400 \mathrm{ft}^{2} /$ day is used in the south. A block of lower transmissivity southeast of the A/M Area is present in both layers 2 and 3. A transmissivity of $4,000 \mathrm{ft}^{2} /$ day was calculated for the Black Creck (Figure 18).

Tho leakance values for layer 1 are very similar to the values used by Papadopulos (1987; Figure 19). Leakance coefficients range from 0.00018 to 0.002 day $^{-1}$. The lowest leakance value is in the A/M Area, and the highest leakance value is northeast and east of the A/M Area. Leakance values for layer 2 range from 0.000403 to 0.22 day $^{-1}$ (Figure 20). Leakance is higher in the south than in the north. The range in leakance values for layer 3 is 0.000002 to 0.00055 (Figure 21). The lowest values are in the $\mathrm{A} / \mathrm{M}$ Area and southeast of the $\mathrm{A} / \mathrm{M}$ area. 


\subsubsection{Recharge}

An uneven distribution of rainfall recharge is chosen for this model. A recharge rate of $15 \mathrm{in} / \mathrm{yr}$ is used over the top of the ridge and a lower rate of $10 \mathrm{in} / \mathrm{yr}$ is used in areas of steeper topography (Figure 22).

\subsection{MODEL SIMULATION OF GROUNDWATER WITHDRAWALS}

Present production and recovery well pumpages in the $\mathrm{A} / \mathrm{M}$ Area are included in this model. Appendix $D$ is a table of the well positions and withdrawal rates provided by SRL for the period to which the model was calibrated. The recovery wells and four of the production wells located in the $\mathrm{A} / \mathrm{M}$ Area are show $\mathrm{n}$ in Figure 3. Production well PW-67B is located in the southern portion of the model southeast of the intersection of Raads $C$ and 2. Wells PW-112G and PW-113G are located east of Tim's Branch and the A/M Area. The seven production wells pump from Aquifer System $\mathrm{I}$. Because this model does not simulate the groundwater flow system below the Black Creck, pumpages were pro-rated according to transmissivity. Therefore, the anticipated amount of pumpage contributed from the Black Creck assumed to be onc-third of the total pumpage.

The recovery wells are screened in the water table, Upper Congaree and Lower Congarec and pumpage is proportional to transmissivity in the three layers. The water table contributes $5 \%$ of the total pumpage while the Upper and Lower Congaree contribute $63 \%$ and $32 \%$, respectively. The withdrawal rate from each layer for each recovery well is shown in the table in Appendix $D$. 


\subsection{MODEL CALIBRATION}

The model was considered to be calibrated when: 1) the predicted water levels in each layer approximately matched the averaged observed water levels and gradients; 2) the discharges calculated from the GHB nodes were within the range of observed base flow discharges; and 3) aquifer parameters were within reported ranges. The model was run under steady-state conditions. It is assumed that the drawdown from the present day recovery system has reached equilibrium. The model was also run under transient conditions for periods of one and two years. Model-predicted groundwater level elevations approached steady-state levels. Recharge and well pumpage do not change throughout the simulation. The following section is a brief description of the steps taken in the calibration of this model. A more detailed description of the calibration process is provided in Appendix E.

\subsection{DESCRIPTION OF CALIBRATION PROCESS}

SDI started with the model parameters used in the 1987 SSP\&A calibration; the only deviation from the SSP\&A model was combining the Ellenton and Lower Congaree layers into one model layer and utilizing a leakance coefficient to simulate the Ellenton as a confining unit below the Upper Congaree. The water levels predicted by the SSP\&A model were reproduced in the water table, Upper Congaree, Lower Congarce and Black Creek.

The 1989-1990 observed water levels differ from the model-predicted water levels produced by the SSP \& A, 1987 calibrations. This may be attributed to the effects 
of several years of recovery well pumpage and differences in rainfall rates. The 1987 model-predicted water levels were higher than the 1989-1990 observed water levels.

Additional monitoring wells have been installed since the SSP\&A calibration and understanding of the hydrogeologic system has improved. SDI analyzed the avallable data and recalibrated the model to $1989-1990$ observed water levels, surface water flow, and aquifer test data. Model parameters were adjusted to the final configurations of transmissivity, leakance, recharge and boundary conditions presented in the previous section. A detailed description of the calibration process is presented in Appendix E.

\subsection{CALIBRATION TO OBSERVED WATER LEVELS}

The resulting model-predicted water levels for each layer are shown in Figures $23,24,25$ and 26 . For ease of comparison, the observed water level contours for each layer are shown in red while the model-predicted contours are shown in blue.

\subsubsection{Predicted Water Levels, Water Table}

As shown in Figure 23, the highest water levels are in the vicinity of the $A / M$ Area for both calculated and observed water levels. Although the model-predicted water levels are higher in the A Area than the observed data, the water level gradient agrees well with the observed gradient within the southern sector. The general direction of horizontal flow is to the south and west as well as southeast into Tim's Branch. The predominant direction of groundwater flow in the water table is downward into the Upper Congaree. The absence of data points east of Tim's Branch made calibration along the northeast boundary of layer difficult. 


\subsubsection{Predicted Water Levels, Upper Congaree}

The model-predicted water levels are a few feet lower than the observed water levels north of the A Area (Figure 24). The general direction of groundwater flow is to the southeast towards Upper Three Runs Creek and to the southwest towards the wetlands. Overall, the agrecment between the model-predicted water levels and the observed water levels is good.

\subsubsection{Predicted Water Levels, Lower Congaree}

As shown in Figure 25, the calculated water levels are slightly higher in the area south of Lost Lake. The northward inflection in the 220-foot contour line is not as pronounced in the model-predicted water levels as it is in the observed water levels. This may be an artifact of model discritization due to a change in aquifer parameters or the use of averaged pumpage rates. Overall, the agreement between the modelpredicted water levels and the observed water levels is good in layer 3.

\subsubsection{Predicted Water Levels, Black Creek}

As shown in Figure 26, calculated water levels agree well with observed water levels in the northern half of the model. Calculated water levels are about 3 fect lower in the vicinity of the only southern data point (well P-29). Because there is little water level data in the southern half of the model area, the observed contours were taken from a regional water level map of the Black Creck. The data used in contouring the regional map are too coarse to show any detail in the Upper Three Runs Creck area. 
The model indicates that there is some upward flow of groundwater from the Black Creek into the Lower Congaree in the southern half of the model due to lower hydraulic heads along Upper Three Runs Creek.

\subsection{CALIBRATION TO SURFACE WATER FLOW}

In order to calibrate the model to surface water flow, stream discharge data were used to estimate the base flow in Tim's Branch and Upper three Run's Creek. Groundwater flow out of the GHB nodes was adjusted until it was within the range of base flow estimated for each stream reach.

Discharge data for Tim's Branch in WY 1987 indicates the base flow averages 3 to $5 \mathrm{crs}$. Because approximately $43 \%$ of the Tim's Branch drainage basin is within the model area, 1.3 to $2.2 \mathrm{cfs}$ of the base flow is assumed to be contributed by the model arta. The discharge through the GHB nodes simulating Tim's Branch in the model is $3.4 \mathrm{cfs}$. This is slightly higher than the estimated contribution for the model area. However, the flow in the upper portion of Tim's Branch is reported to be seasonal so a larger perecntage of the base flow may be contributed by lower Tim's Branch.

The gauging station at Road $A$ and Upper Threc Runs Creck had an estimated base flow of 120-200 cfs in WY 1987. The model area east of the drainage divide is approximately $10 \%$ of the total drainage basin area above the gauging station, thus approximately 12 to $20 \mathrm{cfs}$ of discharge is assumed to be contributed by the model area. The nlow through the GHB nodes simulating Upper Three Runs Creck in the model is $15.2 \mathrm{crs}$. 


\subsection{WATER BALANCE}

In Table 1, the water balance calculated by MODFLOW for this calibrated model is presented. Water enters the model from rainfall recharge, flow into GHB nodes simulating streams, and flow from constant-head nodes simulating water that enters the model area at the boundarics. The total amount of water flowing into the model is $2,880,000 \mathrm{rt}^{3} / \mathrm{day}$, Of this total, rainfall recharge accounts for $78 \%$, while constant-head nodes contribute $17 \%$ and GHB nodes on the upper portion of Tim's Branch account for $5 \%$.

Table 1. Volumetric Budget for Entire Model at End of Time Step 1 in Stress Period 1.

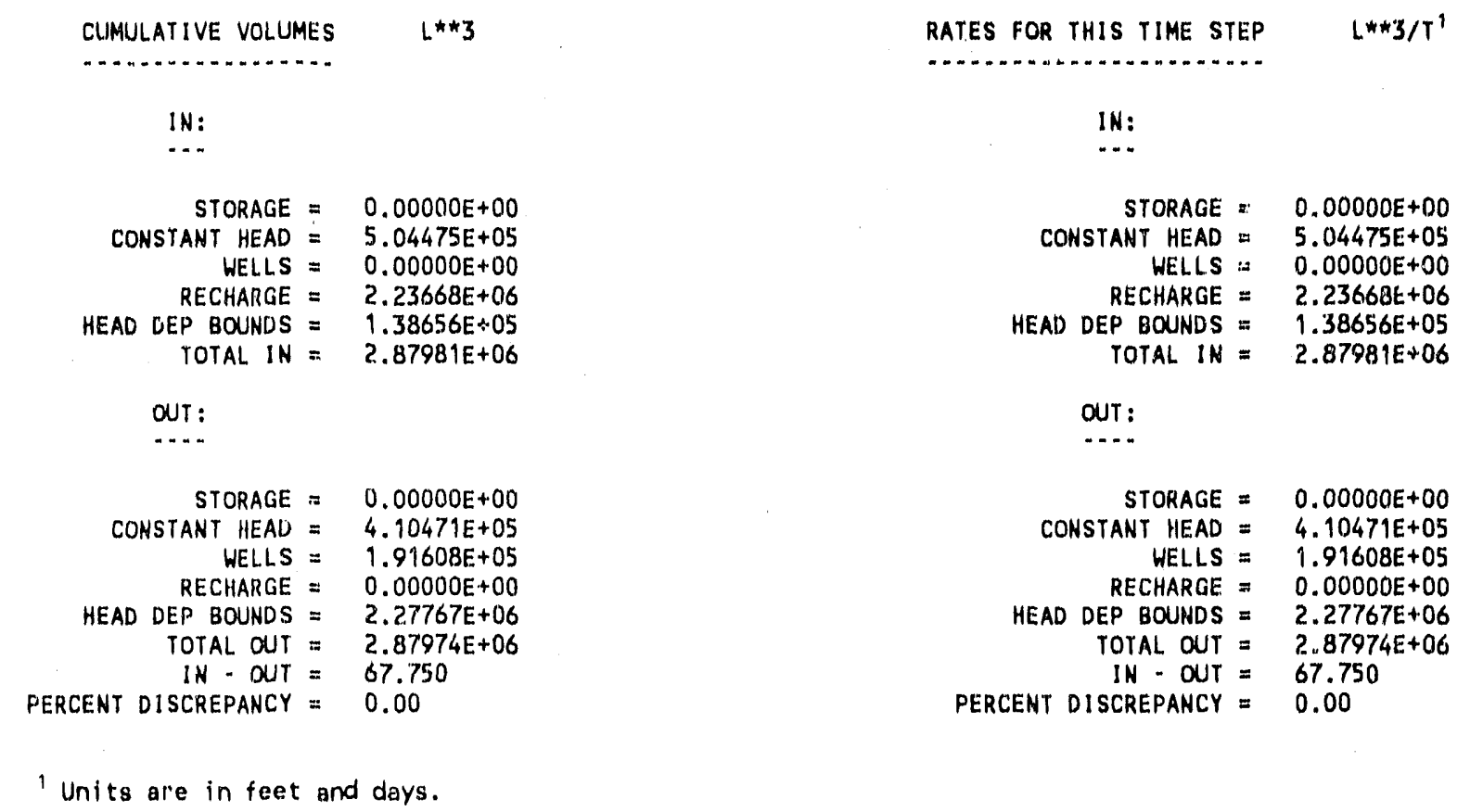


Groundwater exits the model through surface water runoff, groundwater discharge to streams, groundwater flow out of model area boundaries, and well pumpage. The total amount of water flowing out of the model is $2,880,000 \mathrm{ft}^{3} / \mathrm{day}$. Of this total, $79 \%$ exits the model through the GHB nodes simulating surface water runoff. This is an average discharge from the model of $26.4 \mathrm{cfs}$. The amount of groundwater flowing out of the model through boundaries is $14 \%$ and the total pumpage from the recovery and production wells is $7 \%$.

Table 2 is the MODFLOW calculated breakdown of the movement of water into and out of the model by layers. The model output indicates that there is both upward and downward vertical flow between the model layers. The net vertical flow between layers is downward. The largest amount of water moving between layers is downward from layer 1 to layer 2 .

Constant-head boundary nodes allow the simulation of groundwater flowing horizontally into and out of the model. Table 2 indicates that the largest amount of horizontal flow into and out of the model is in layer 4 . With the exception of layer 4 , the net flow at the constant-head boundaries is into the model. Groundwater enters all 4 layers through the northeastern corner of the model.

Of the total amount of water flowing out of the model, $7 \%$ is from well pumpage. Production wells in the Black Creek extract approximately $57 \%$ of the total pumpage. The recovery wells pump $2 \%$ from the water table, $27 \%$ from the Upper Congaree and $14 \%$ from the Lower Congaree.

The total amount of rainfall recharge entering the model is $2,237,000 \mathrm{rt}^{3} / \mathrm{day}$ which averages $11.2 \mathrm{in} / \mathrm{yr}$ over the model area. Layer 1 receives $31 \%$ of the total recharge and layer 2 receives the other $69 \%$. 
Table :. Volumetric Flow Budget for Each Model Layer for Time Step 1 in Stress Period 1.

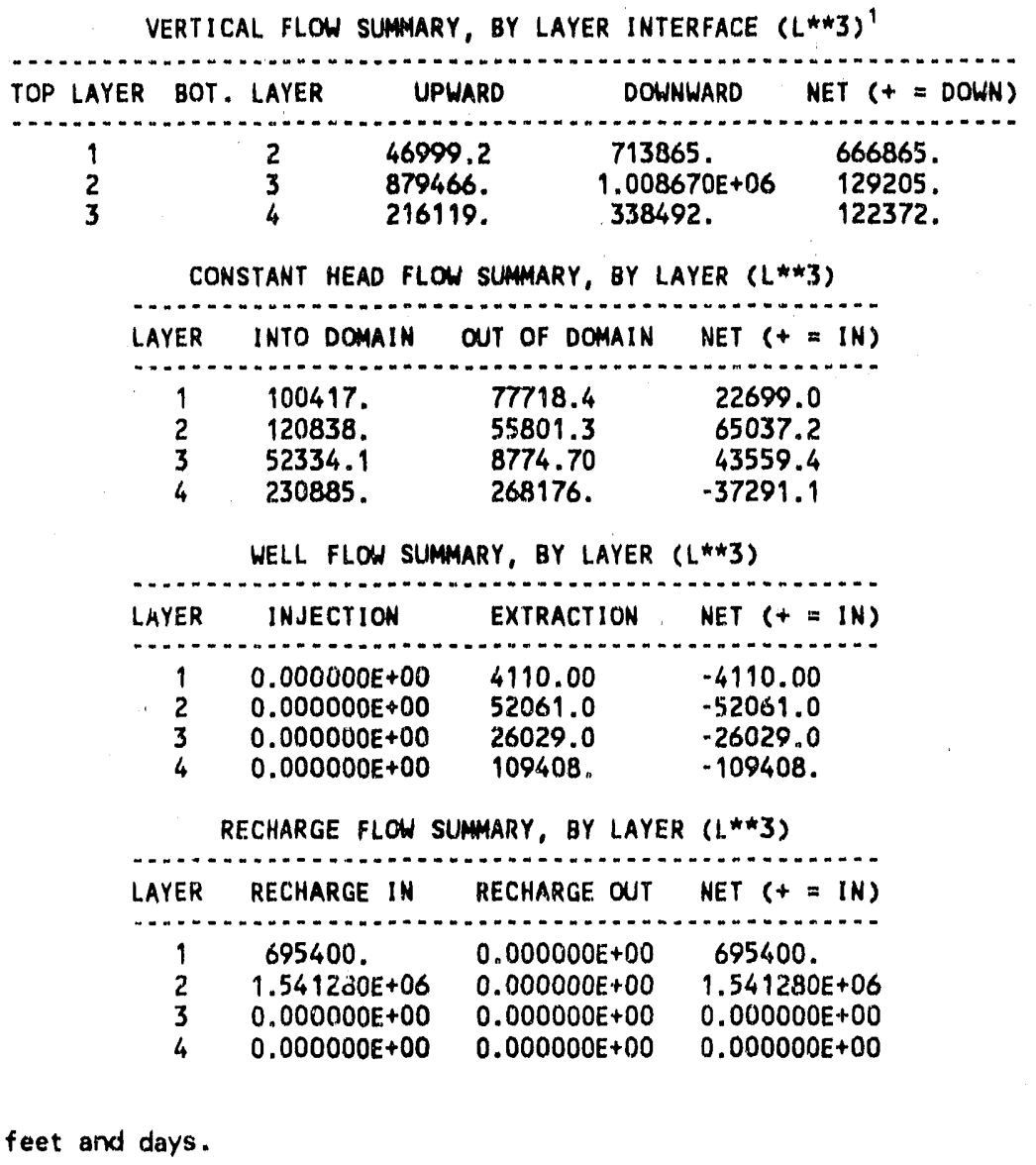

1 Units are in feet and days.

The coarse grid groundwater flow model has been calibrated to the available data. The predicted water levels in each layer agrec favorably well with the observed water level data. The discharge from GHB nodes simulating Tim's Branch and Upper Three Runs Creck are within the ranges of base flow estimated from stream discharge data. Aquifer parameters used in the model are within reported valucs. 


\subsection{FUTURE USE}

The purpose in constructing this model is to evaluate groundwater remediation options and assess recovery systems in the vicinity of the A/M Area of the SRS. This calibrated coarse-grid model is a base model. Specific areas of interest can be fine gridded to provide more accurate water level elevations that can be used for particle tracking and capture zone analysis. Copies of the MODFLOW code and input data have been furnished to WSRC on floppy disk. SDI will use this model to investigate groundwater accovery options for the "southern sector" south of Lost Lake and the A/M Area. 


\subsection{REFERENCES}

Aadland, R.K., and Bledsoe, H.W., 1990. Hydrogeologic Characterization of the Cretaceous-Tertiary Coastal Plain Sequence at the SRS Westinghouse Sivannah River Company.

Cahill, J.M., 1982. Hydrology of the Low-Level Radioactive-Solid Waste Burial Site and Vicinity near Barnwell, South Carolina: U.S. Geological Survey Open-File Report 82-863.

Christensen, E.J., and Gordon, D.E., 1983. Technical Summary of Groundwater Quality Protection Program at Savannah River Plant, Volume 1-Site Gcohydrology, and Solid and Hazardous Wastes, DPST-83-829, E. I. DuPont deNemours \& Company, Savannah River Plant, Aiken, SC.

Dufficld, G.M., Stephenson, D.E., Buss, D.R., and Wadsworth, T:D., 1989. Effects of Heterogencous Porous Geology on Ground-Water Flow and Transpori Modeling in Multiaquifer Systems, DP-MS 88-59. Prepared for the Solving Groundwater Problems with Models Seminar, Indianapolis, IN, February 7-9, 1989.

Department of Energy, 1989. Map of Savannah River Plant, United States Geological Survey, 1:48,000.

Fallaw, W.C., 1990. Draft Report - Subsurface Stratigraphy and Structure of A/M Area.

Fallaw, W.C., and Sargent, K.A., 1986. Subsurface Geology of the A and M Areas of the Savannah River Plant, Aiken, South Carolina. Unpublished Savannah River Laboratory Report, Contract AX715063, Savannah River Laboratory, Aiken, South Carolina, 54 pages.

Geraghty \& Miller, Inc., 1985. Hydraulic Properties of the Tertiary Aquifer System Underlying the M-Area. Prepared for E.I. DuPont deNemours \& Company, Savannah River Plant, 32p.

Hubbard, J.E., 1986. An Update on the SRP Burial Ground Area Water Balance and Hydrology: E.I. DuPont deNemours \& Company, Savannah River Laboratory Report DPST-85-958.

Hubbard, J.E, and Emslic, R.E., 1984. Water Budget for SRP Burial Ground Arca: E.I. DuPont deNemours \& Company, Savannah River Laboratory Report DPST-83742.

Lockwood Grecne Enginecrs, Inc., 1983. Stream Flow and Analysis Study. Prepared for E. I. DuPont deNemours \& Company, Savannah River Plant, Aiken, SC. 
Papadopulos, S.S. \& Associates, Inc., 1987. Hydrogeologic Conditions and Evaluation of Chemical Transport in the Vicinity of the M-Area Settling Basin and Lost Lake. Prepared for E.l. DuPont deNemours \& Company, Savannah River Plant, Aiken, SC.

Papadopulos, S.S. \& Associates, Inc., 1986. Evaluation of the Effectiveness of the MArca Extraction System. Prepared for E.I. DuPont deNemours \& Company, Savannah River Plant, Aiken, SC.

Schreuder, P.J., Davis, P.R., Schwartz, M.A., and Haselow, J.S., 1990. Evaluation of Proposed Recovery Well Scenarios A-Area Savannah River Site. Prepared for Westinghouse Savannah River Company, Savannah River Site, Aiken, SC.

SDI [Letter to J.S. Haselow, Westinghouse Savannah River Company], 1990a (August 2).

SDI [Letter to J.S. Haselow, Westinghouse Savannah River Company], 1990b (August 15).

SDI [Letter to J.S. Haselow, Westinghouse Savannah River Company], 1991 (March 19).

USGS, 1987. Water Resources Data, South Carolina; Water Year 1987, U.S. Geological Survey Water-Data Report.

Walton, W.C., 1970. Groundwater Resources Evaluation; McGraw-Hill Book Company, $664 \mathrm{p}$.

Westinghouse Savannah River Company, 1990. Trichloroethylene Postings (4Q90) for Water Table, Upper Congaree, Lower Congaree, and Black Creck, Groundwater Monitoring and Corrective Action Program, M-Arca Hazardous Waste Management Facility Post-Closure Care Pcrmit, 4 TCE Contour Maps. 

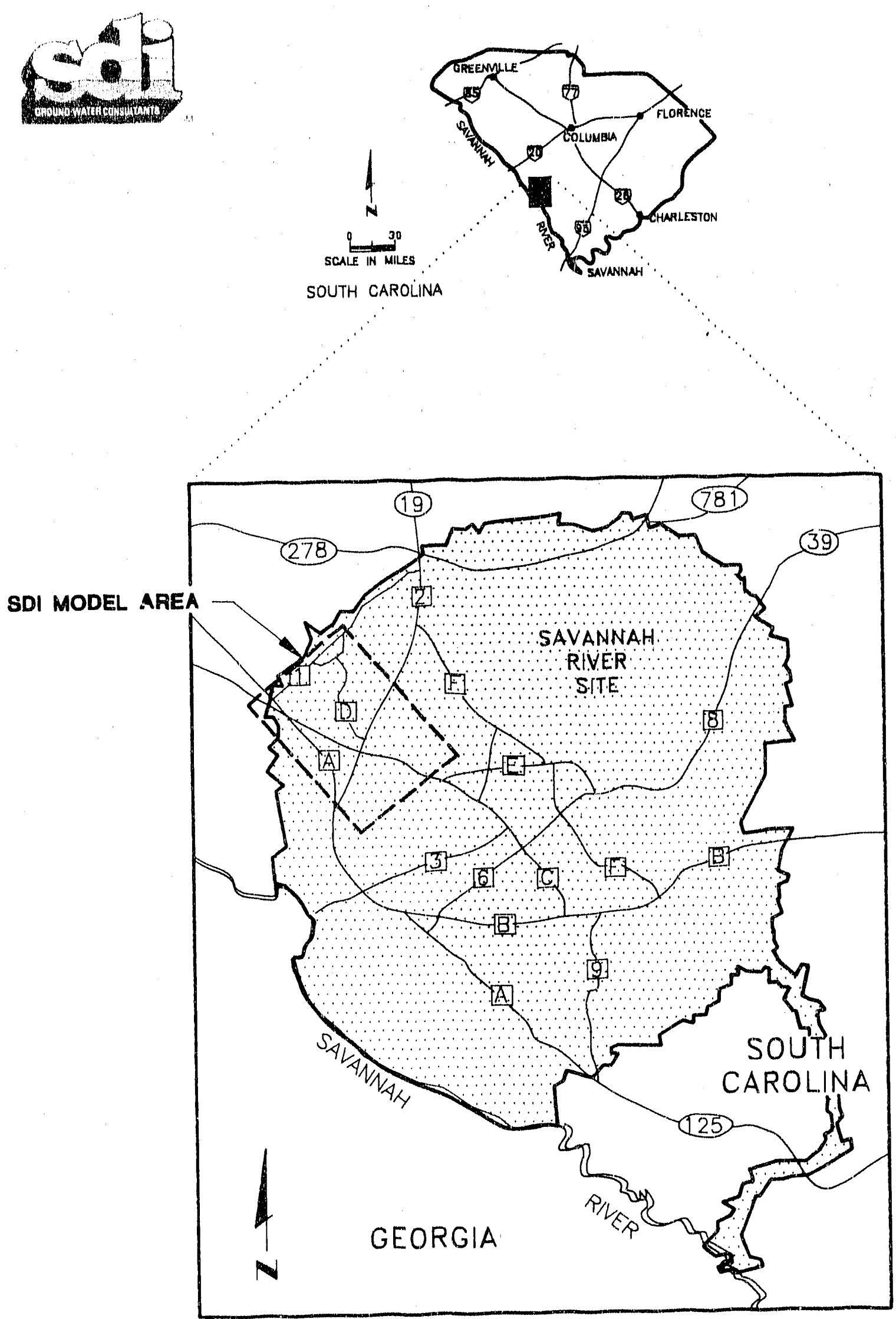

0

Figure 1. Location of the Savannah River Site and Modeled Area.

A Ground Water Flow Model for the A/M Area of the SRS (U) 

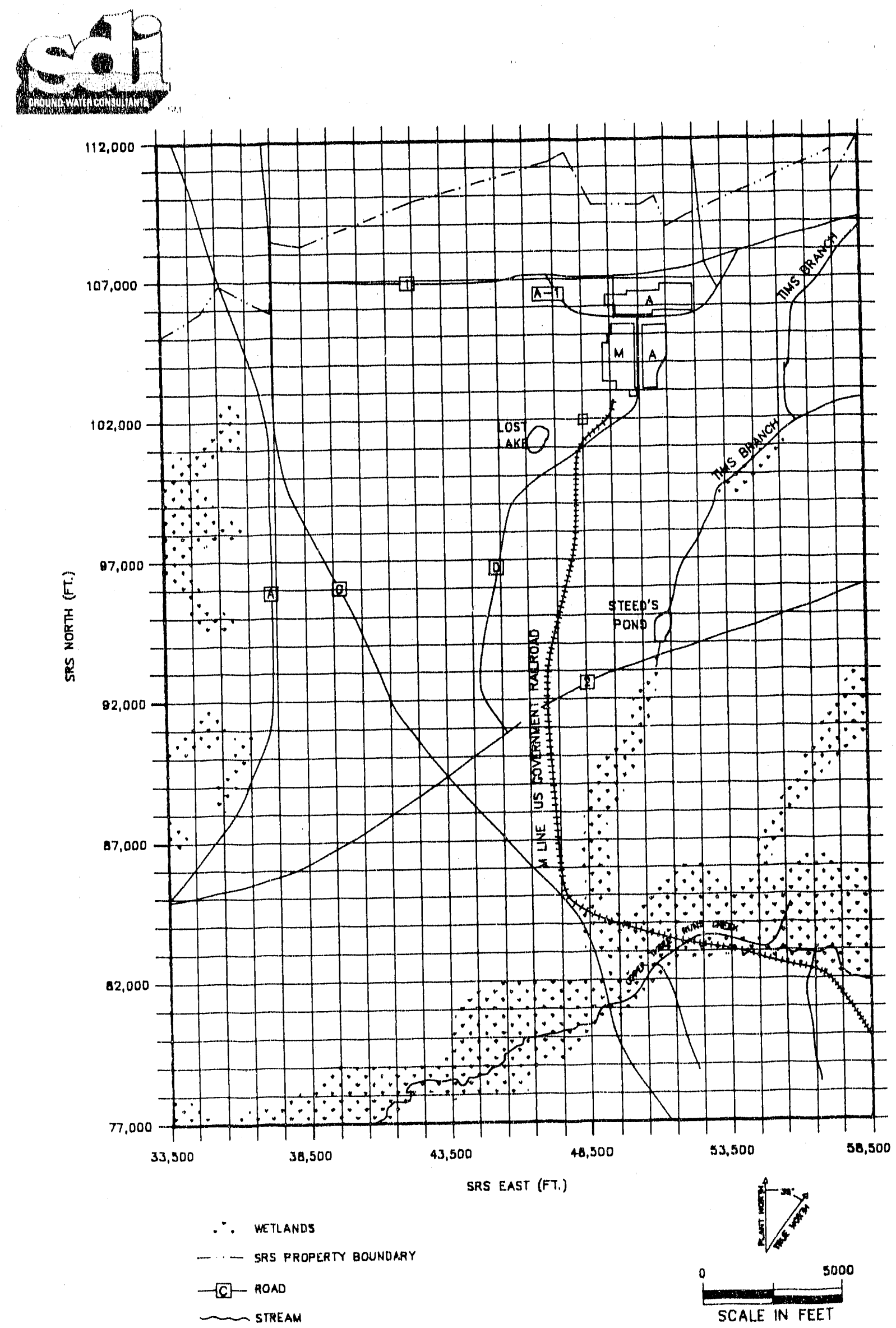

Figure 2. Model Area and Nodal Grid.

A Ground Water Flow Model for the A/M Area of the SRS (U) 


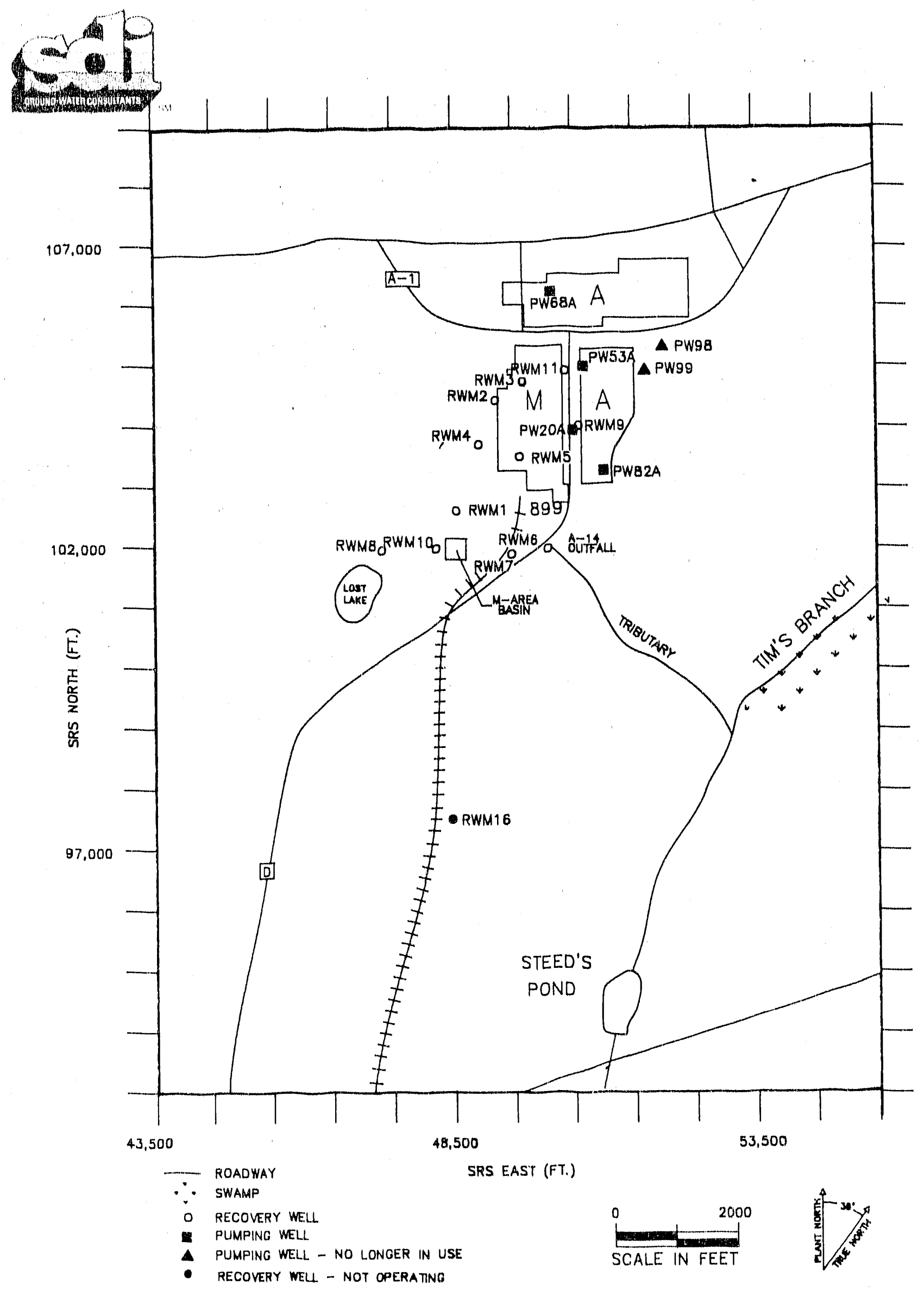

Figure 3. Location of Recovery Wells and Production Wells. 


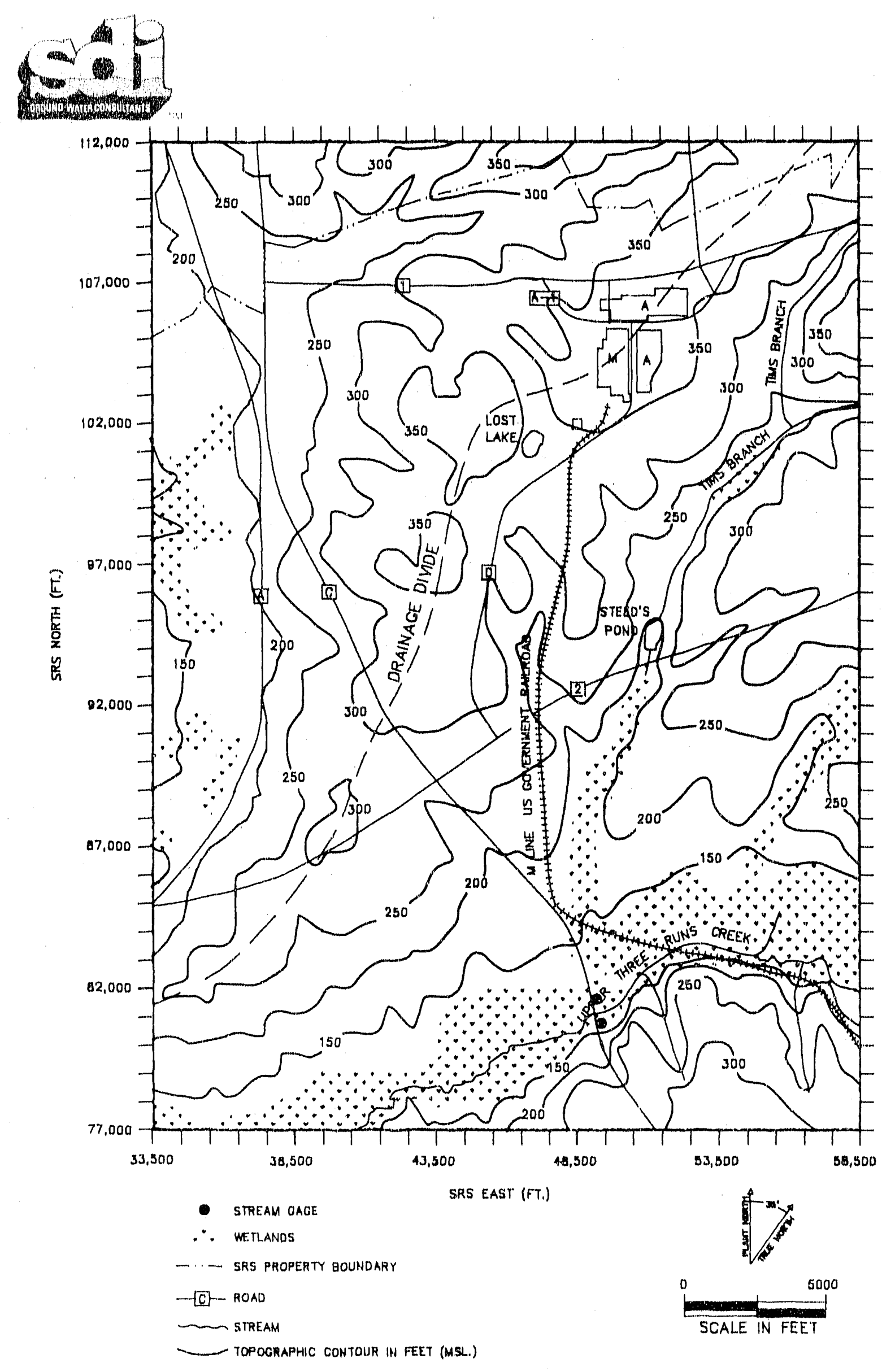

Figure 4. Topography, Watershed Boundary and Surface Water Features. 


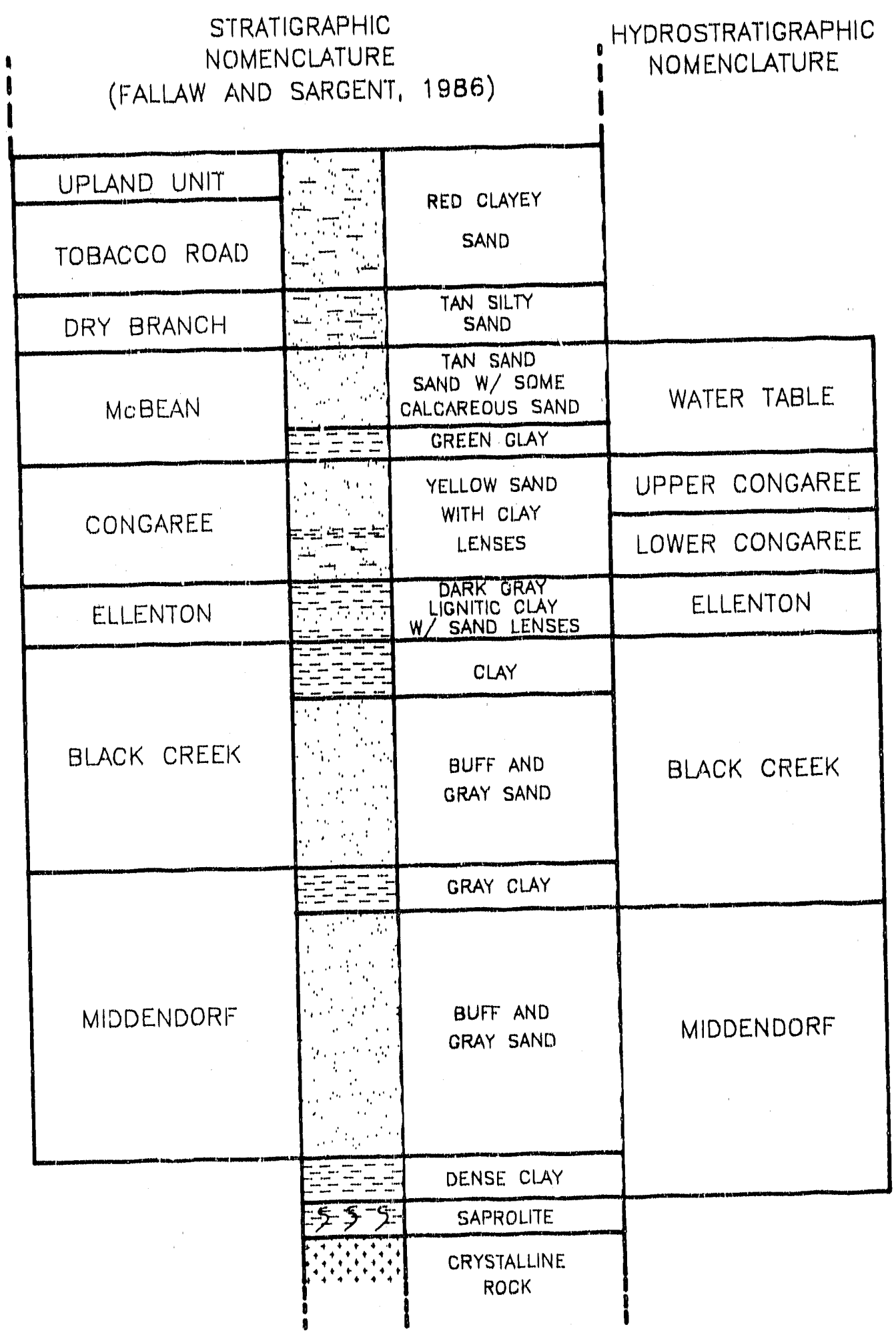

Figure 5. Lithology and Relationship Between Hydrostratigraphic and Stratigraphic Nomenclature. 

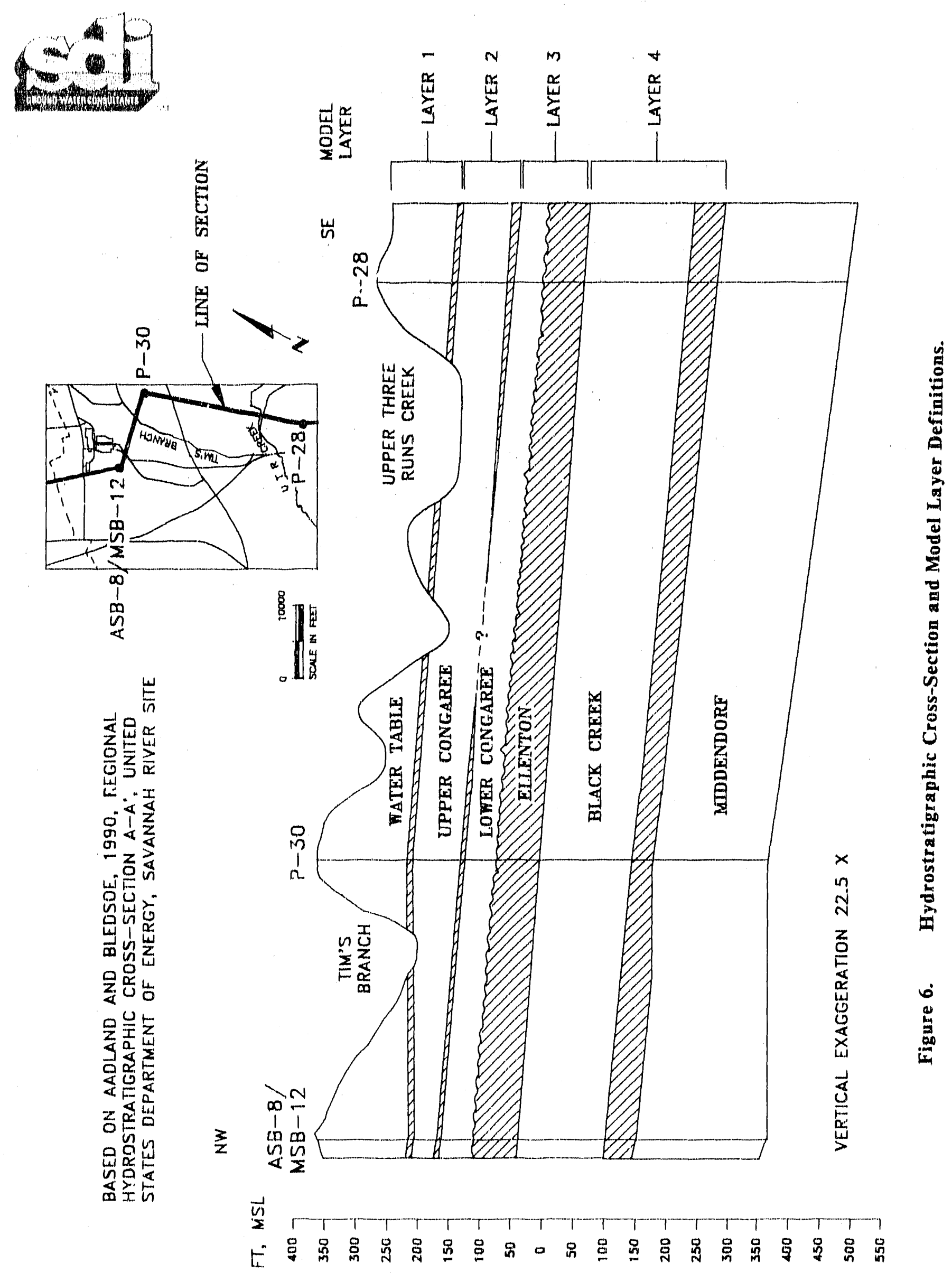

A Ground Water Flow Model for the A/M Area of the SRS (U)

Page 33 

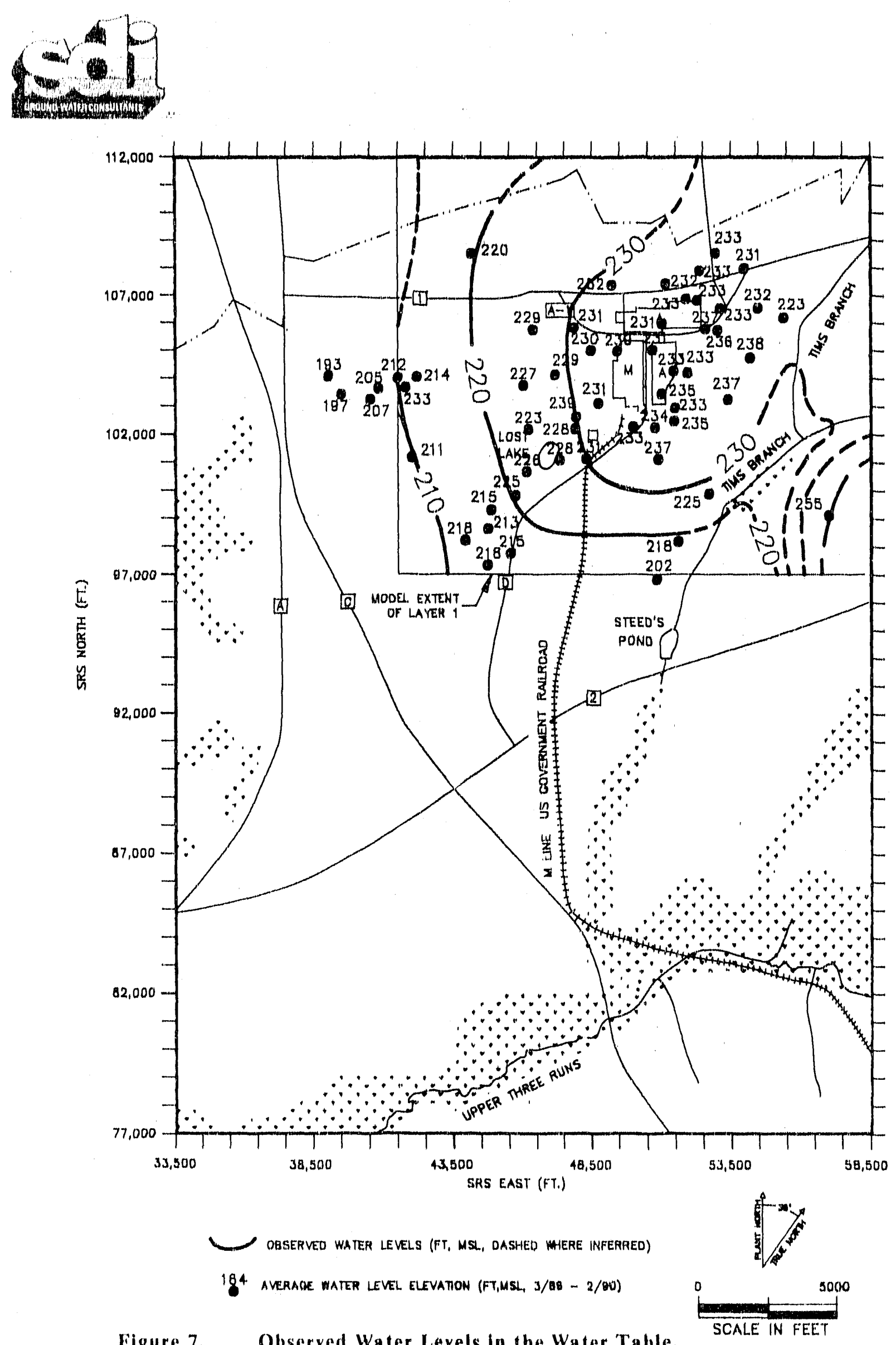

Figure 7. Observed Water Levels in the Water Table.

A Ground Water Flow Model for the A/M Area of the SRS (U)

Page 34 



Figua 8. Observed Water Levels in the Upper Congaree.

A Ground Water Flow Model for the A/M Area of the SRS (U) 

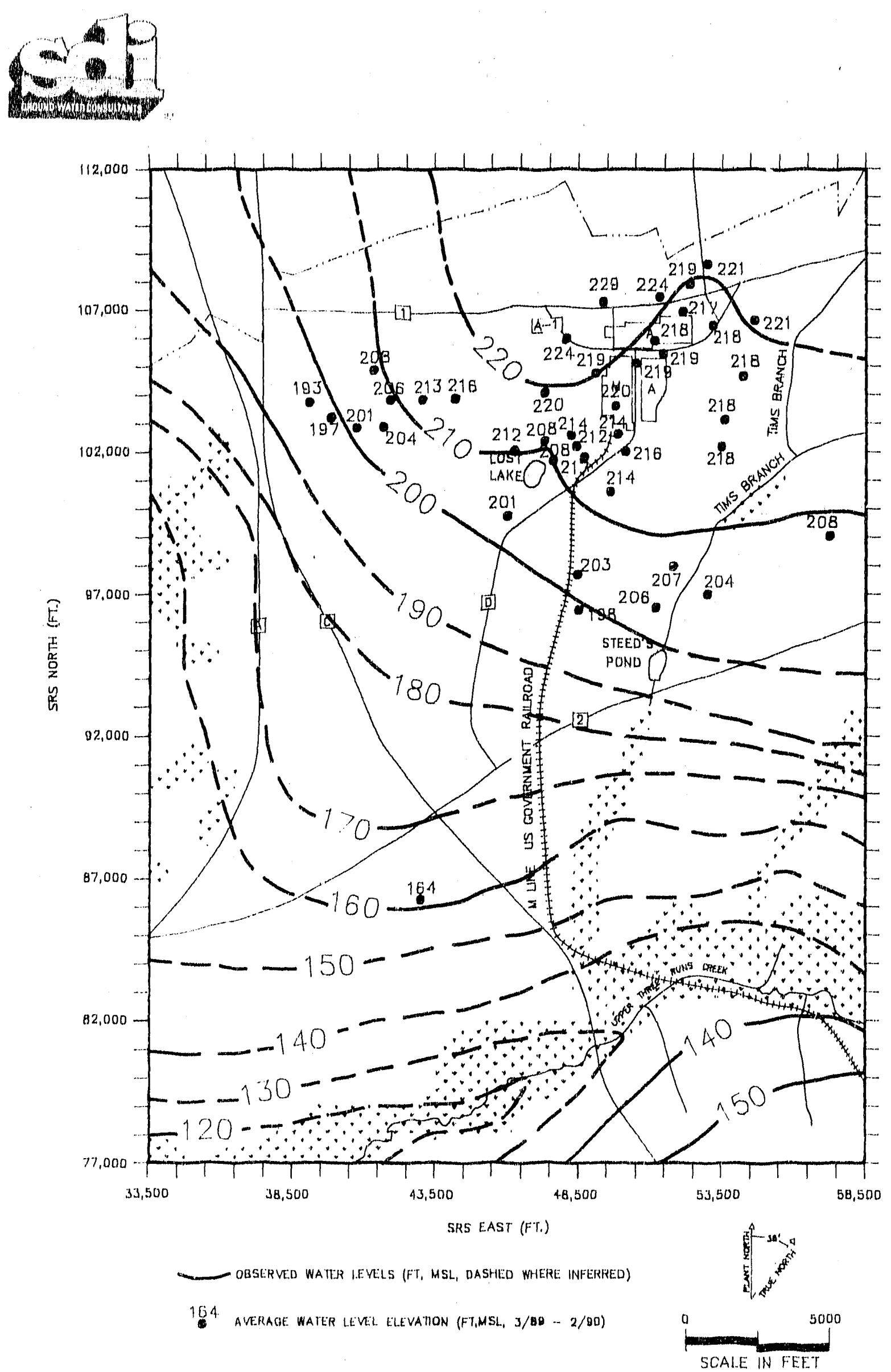

Figure 9. Observed Water Levels in the Lower Congaree.

A Ground Water Flow Model for the A/M Area of the SRS (U)

Page 36 

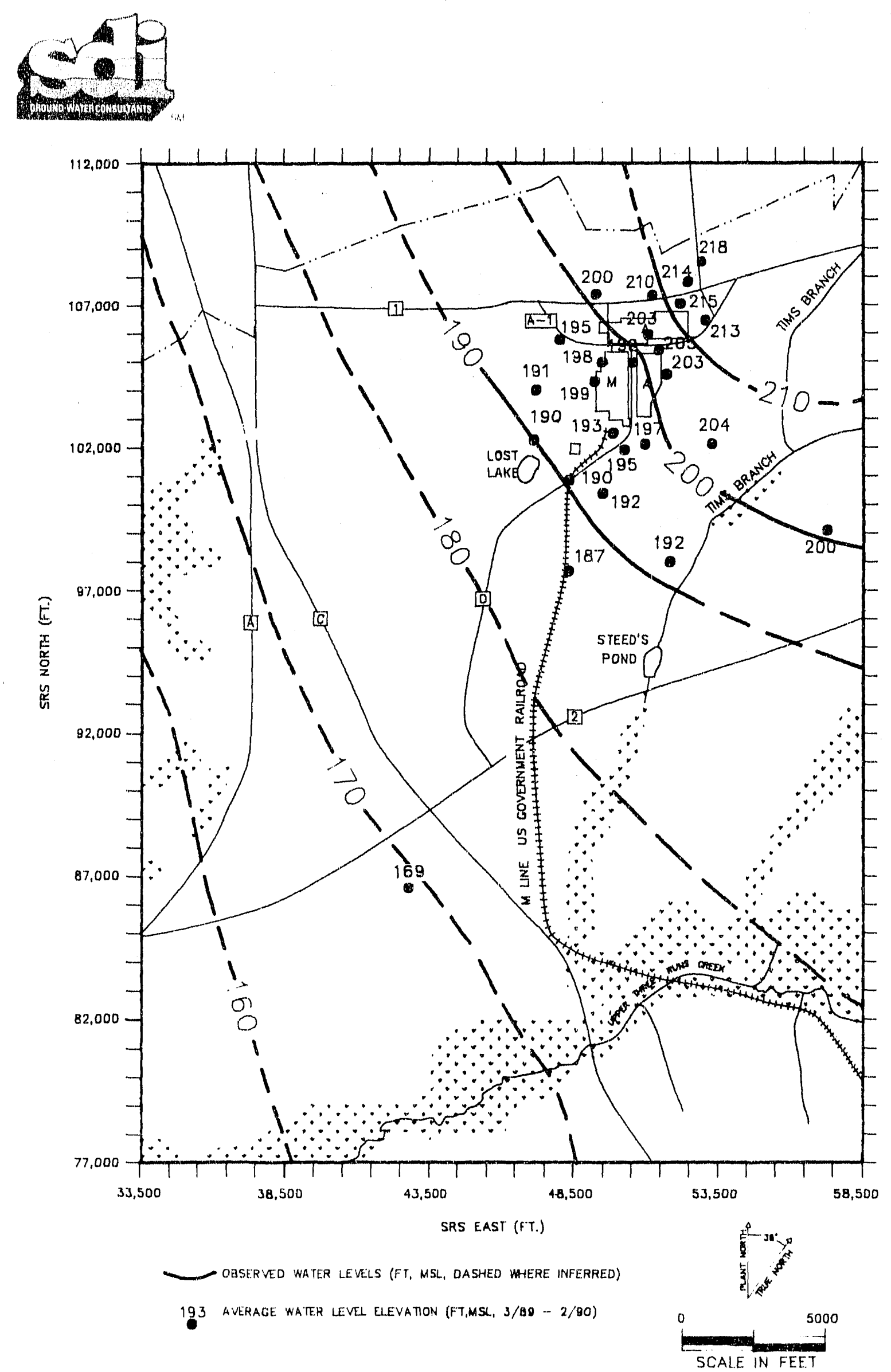

Figure 10. Observed Water Leveis in the Biack Creeh. 

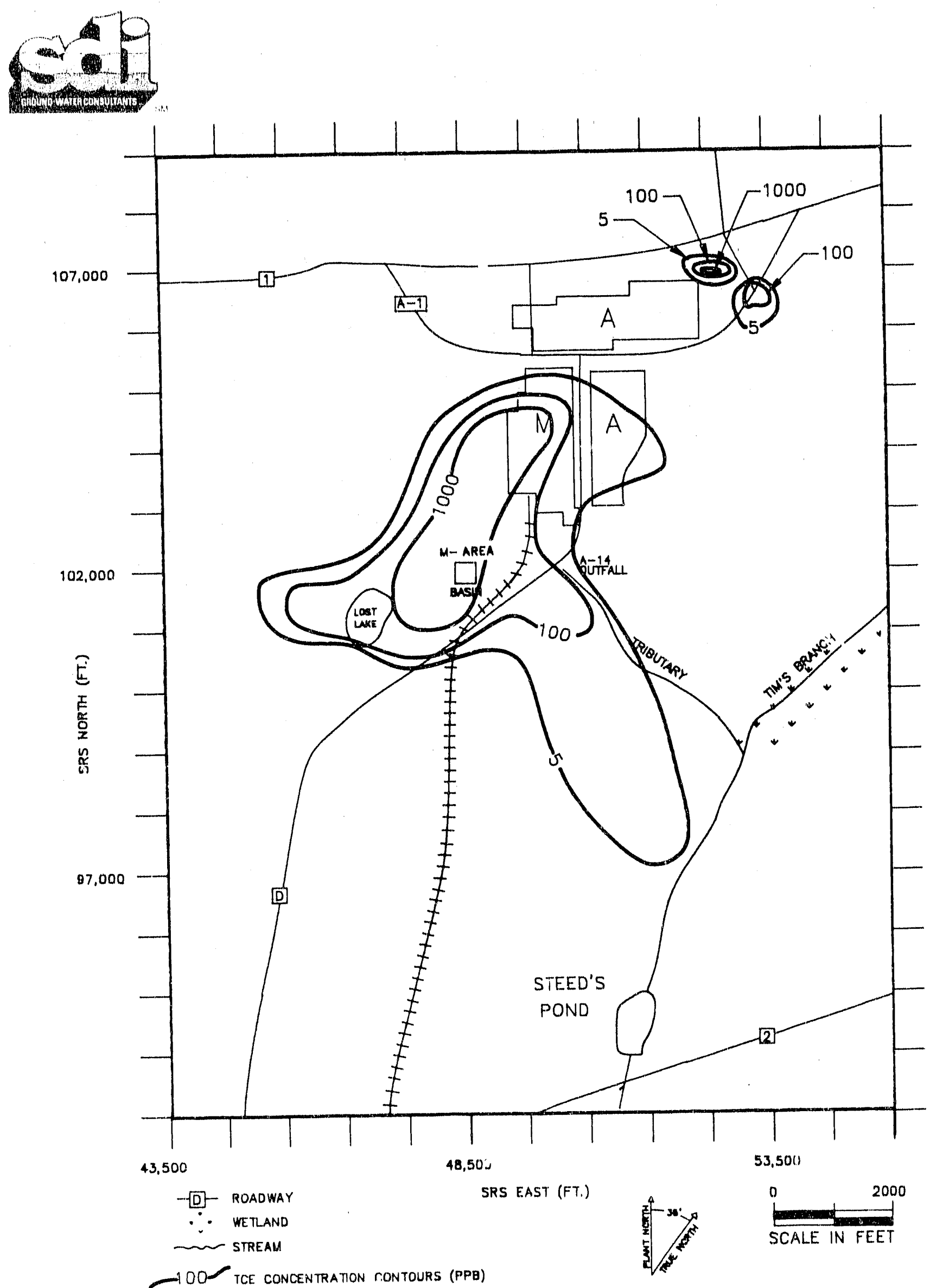

Figure 11. Ohserved TCE Concentration in Water Tabie. After Westinghouse Savannah River Company, 1990. 


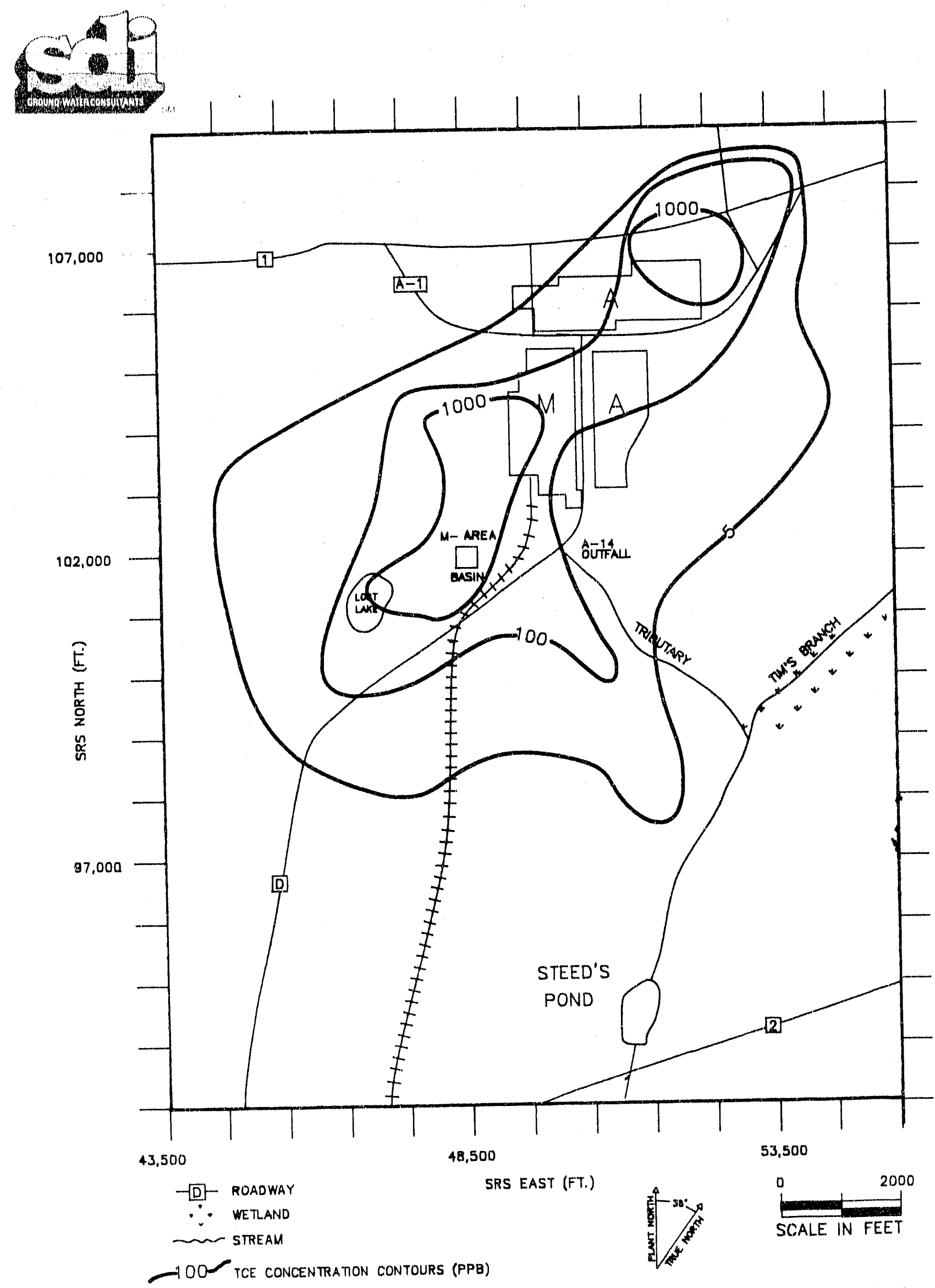

Figure 12. Observed TCE Concentration in Upper Congaree. After Westinghouse Savannah River Company, 1990.

A Ground Water Flow Model for the A/M Area of the SRS (U) 


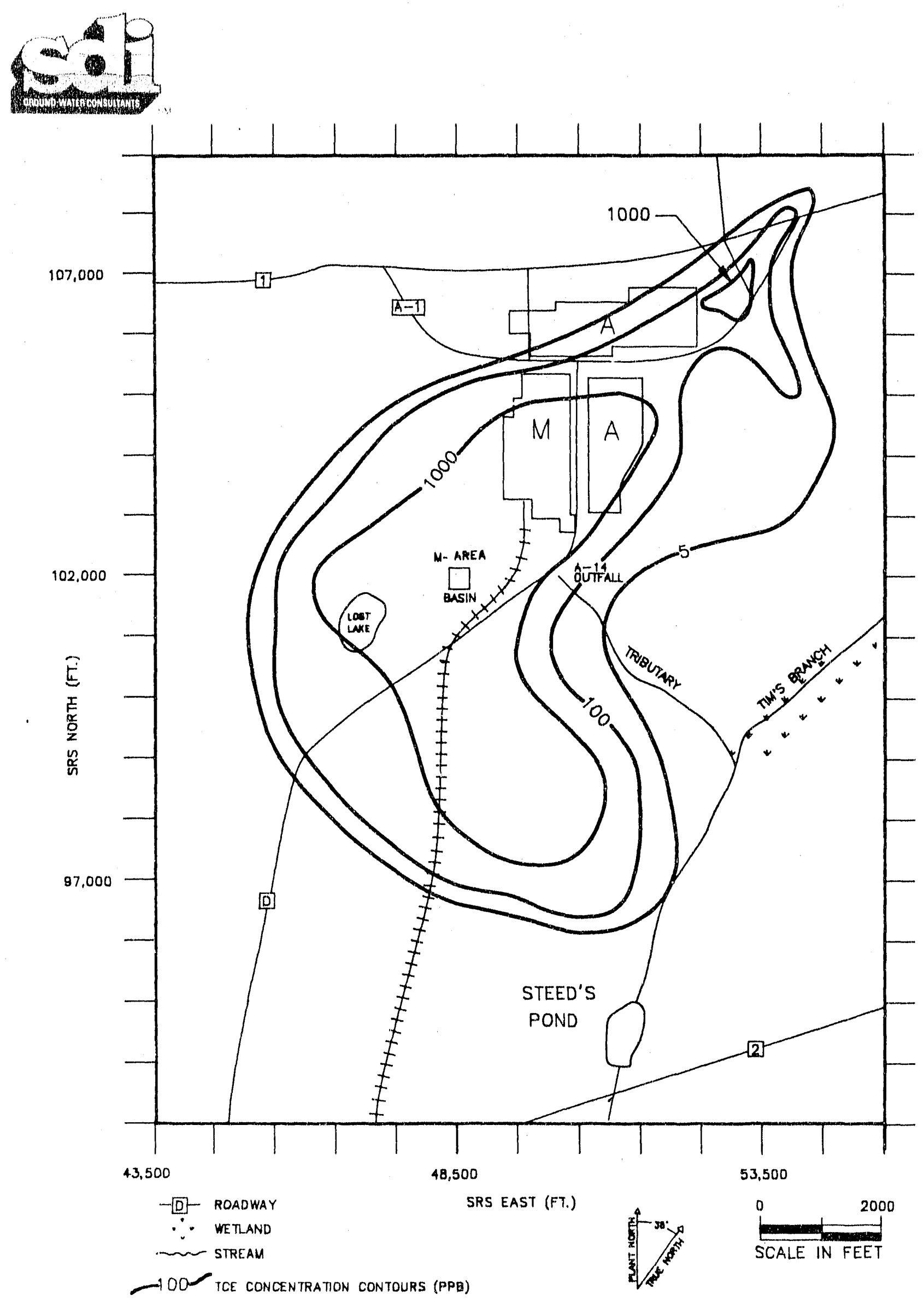

Figure 13. Observed TCE Concentration in the Lower Congaree. After Westinghouse Savannah River Company, 1990. 


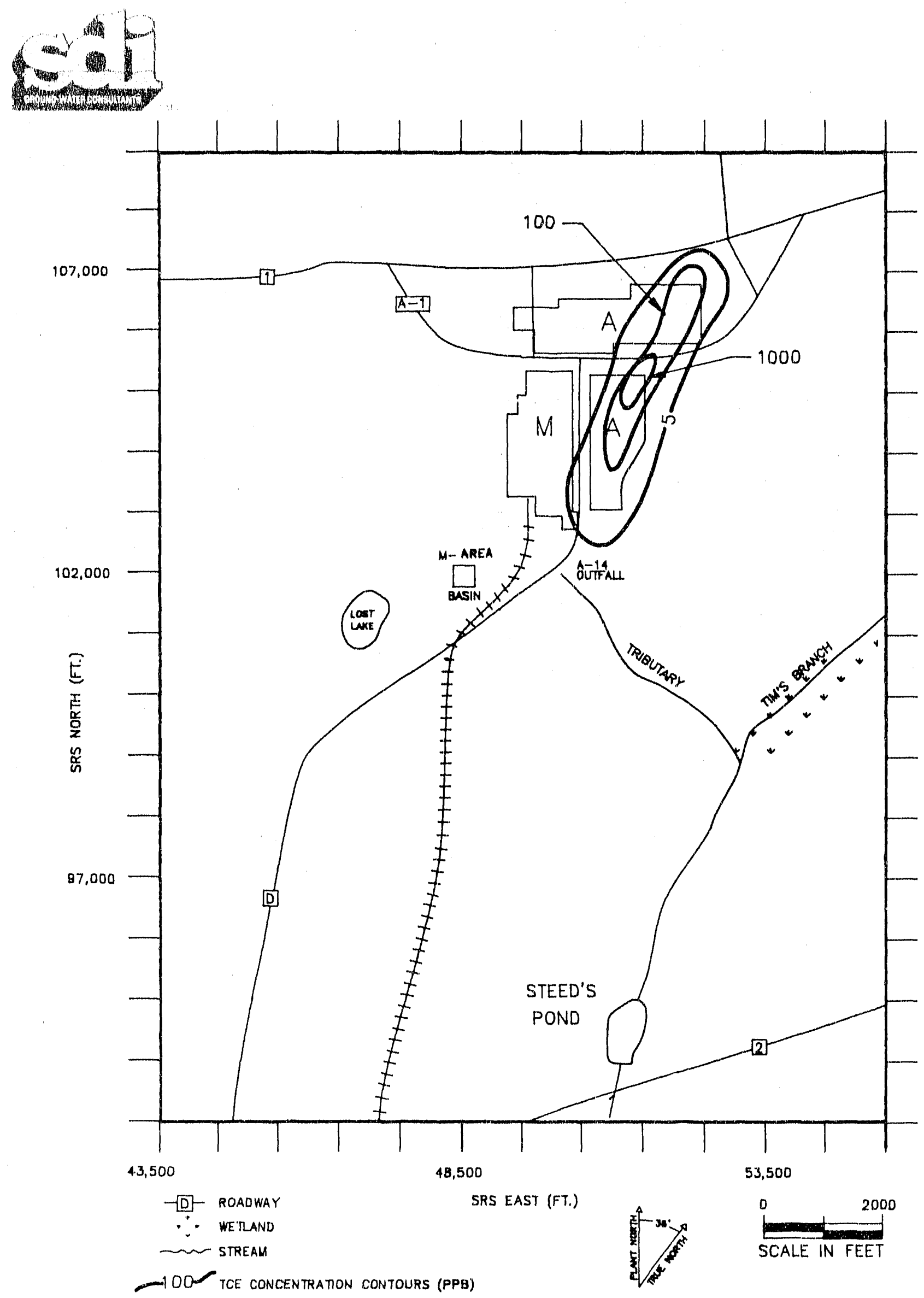

Figure 14. Observed TCE Concentration in the Black Creek. After Westinghouse Savannah River Company, 1990. 

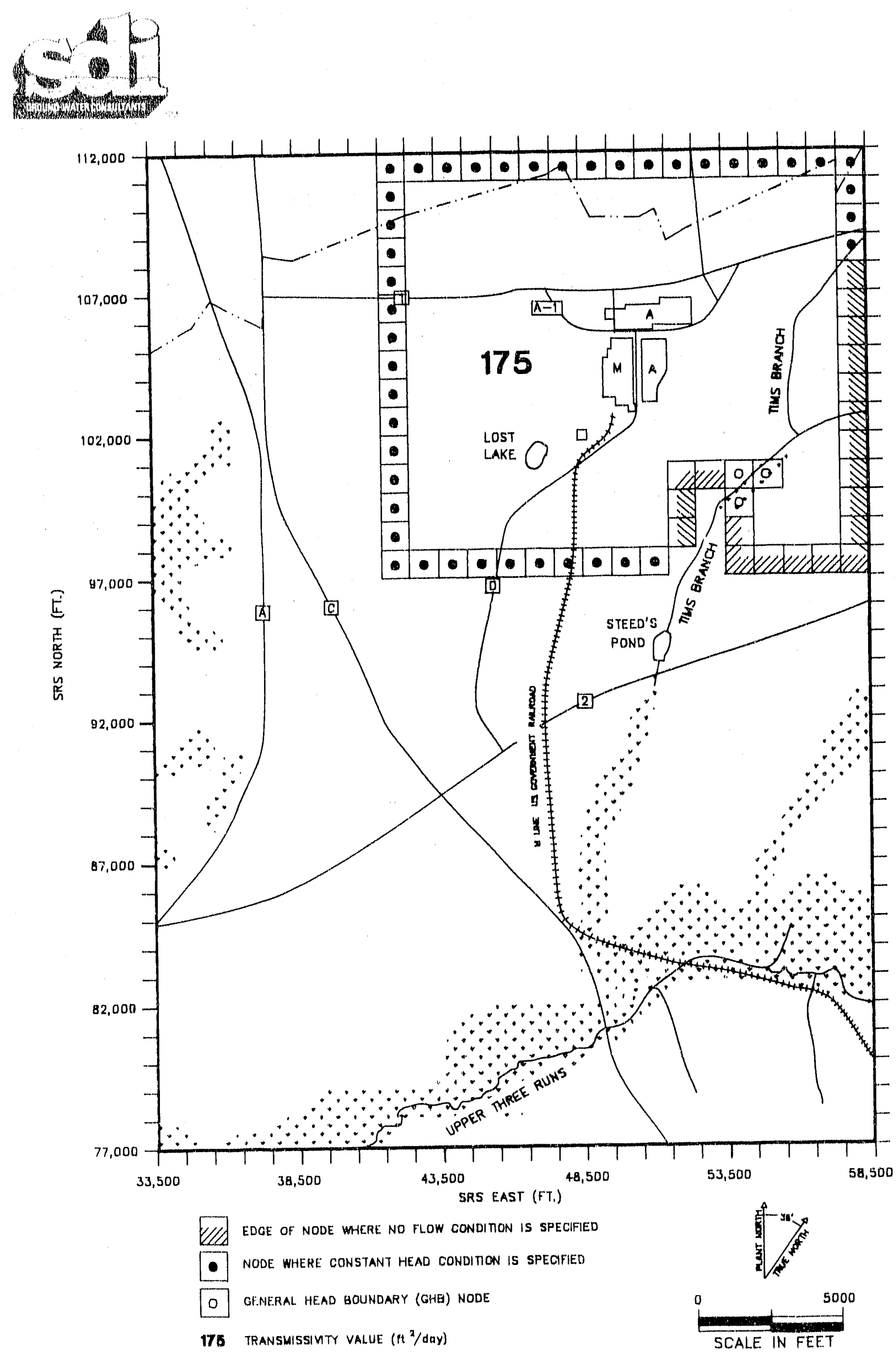

Figure 15. Boundary Conditions and Transmissivity in Model Layer 1. 


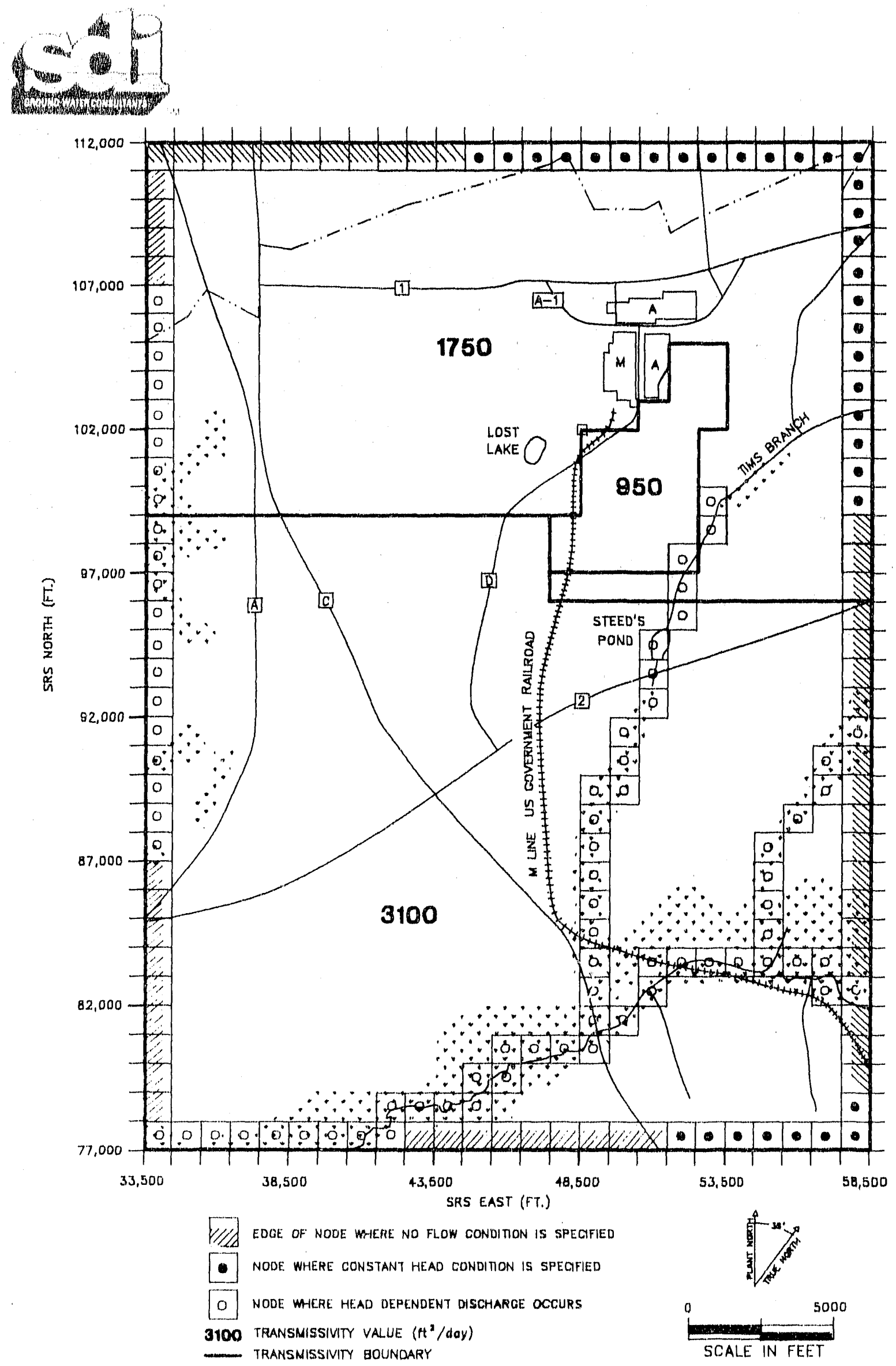

Figure 16. Boundary Conditlons and Transmissivity in Model Layer 2. 


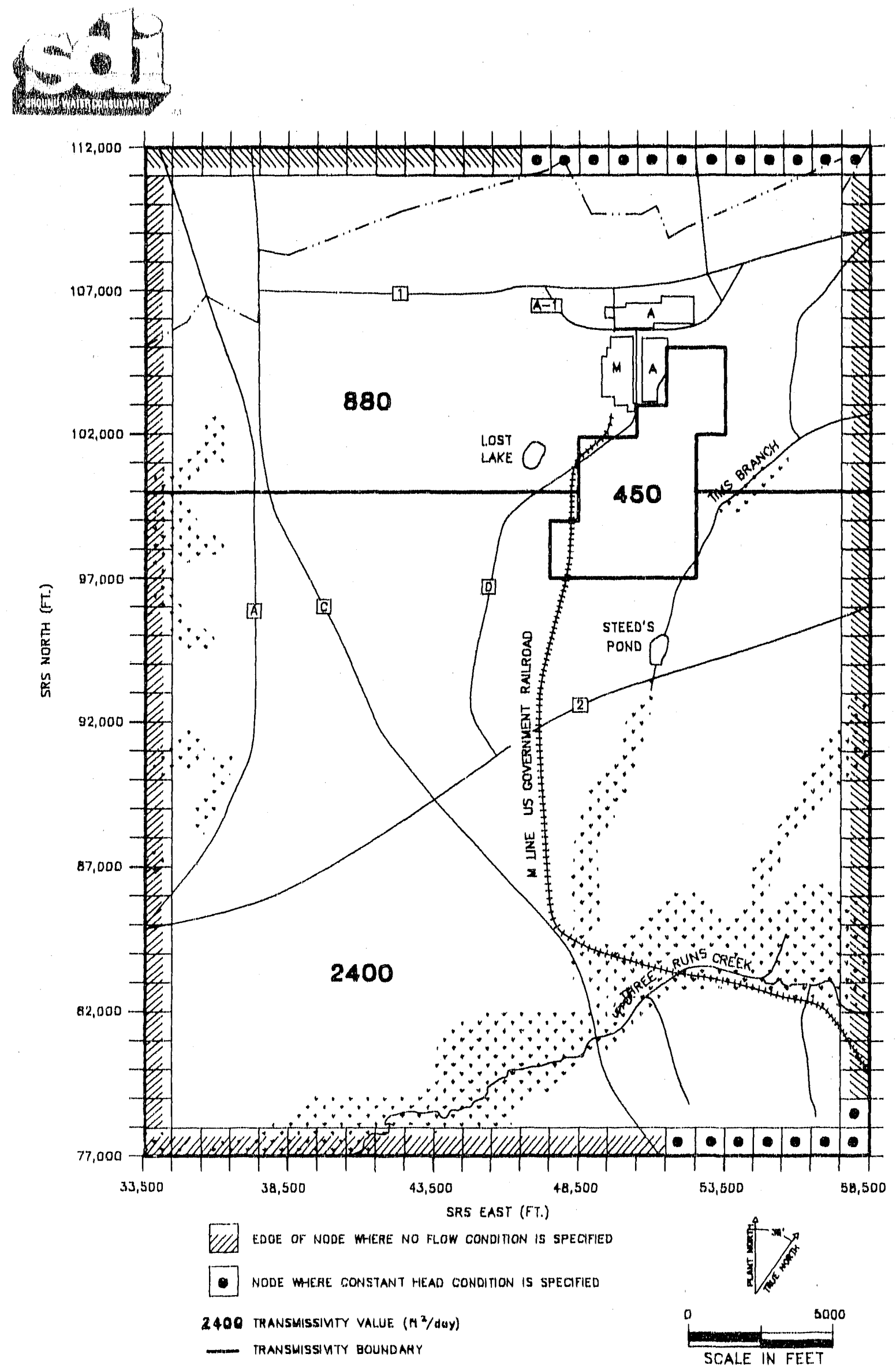

Figure 17. Boundary Conditions and Transmissivity in Model Layer 3.

A Ground Water Flow Model for the A/M Area of the SRS (U) 

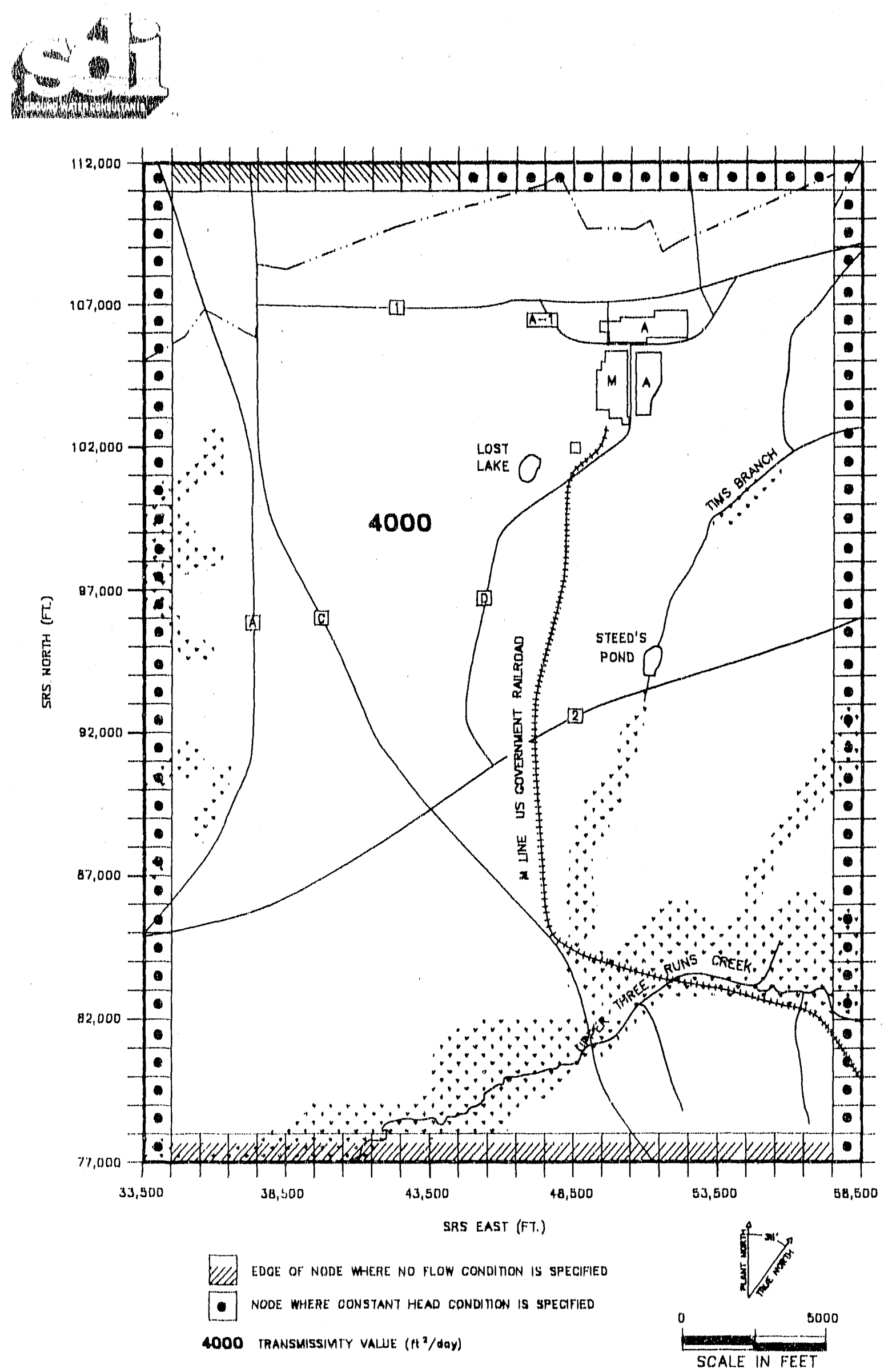

Figure 18 . Boundary Conditions and Transmissivity in Model Layer 4. 


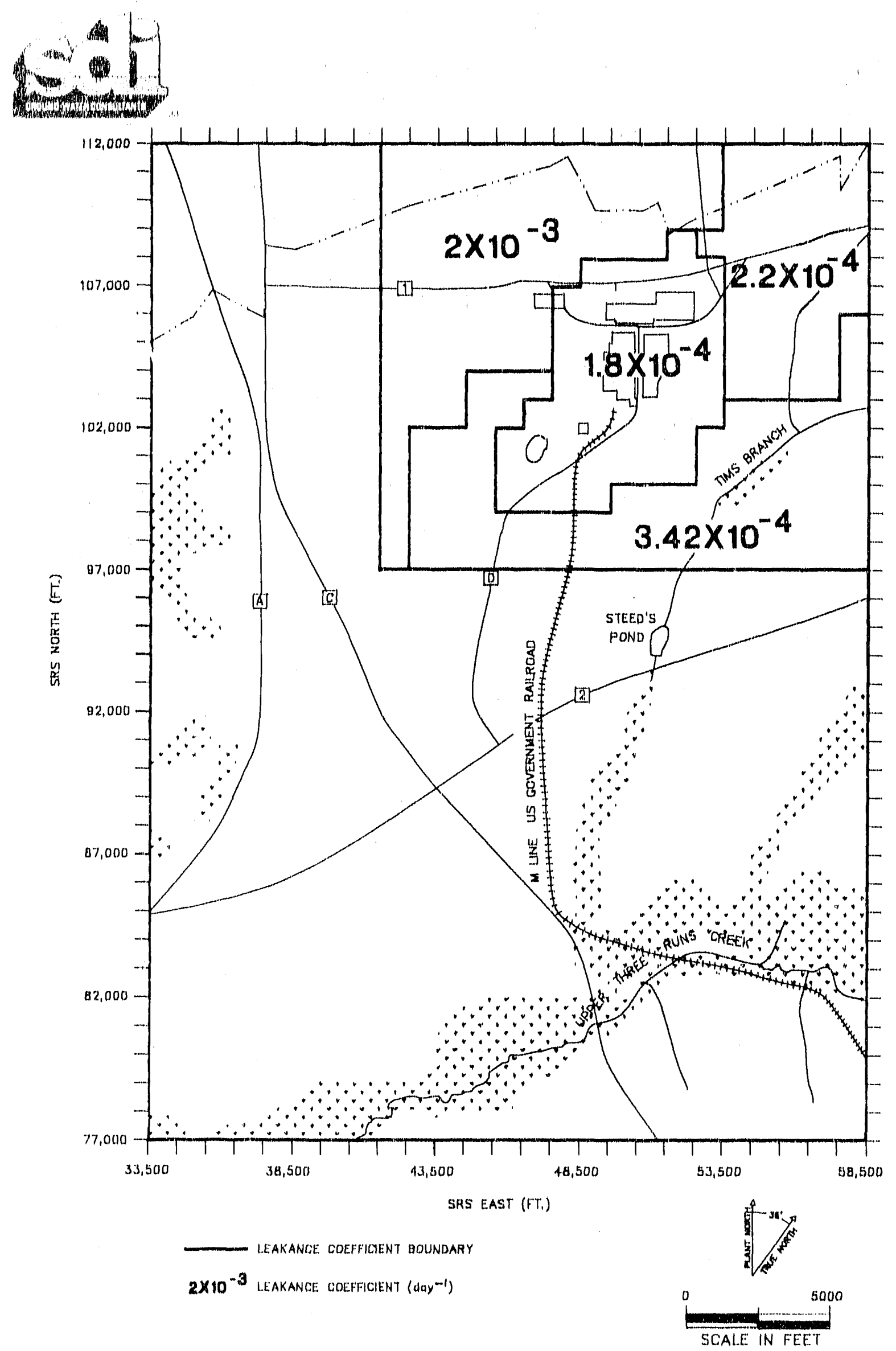

Figure 19. Calibrated Leakance Map for Model Layer 1.

A Ground Water Flow Model for the A/M Area of the SRS (U) 


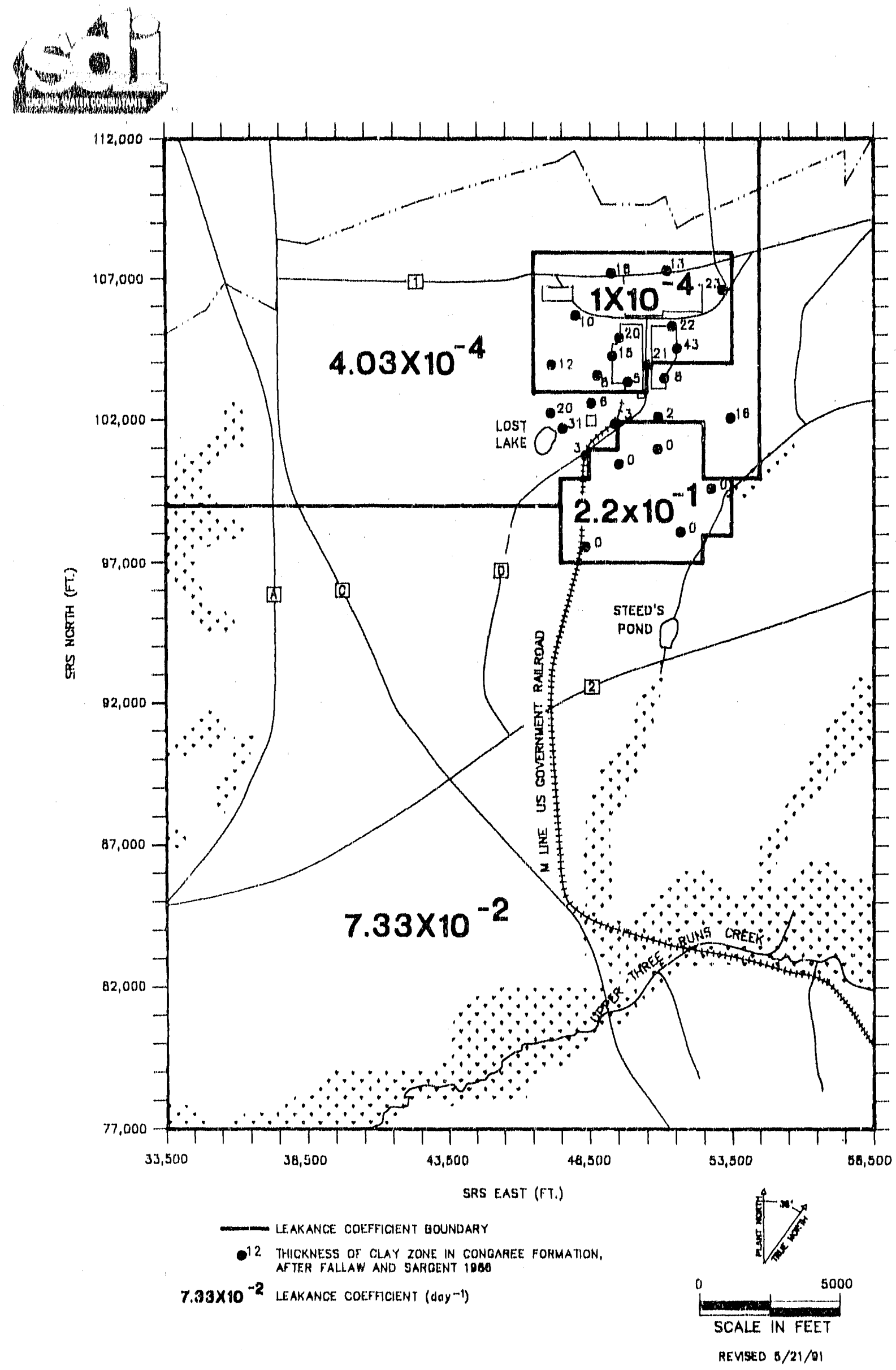

Figure 20. Calibrated Leakance Map for Model Layer 2. 

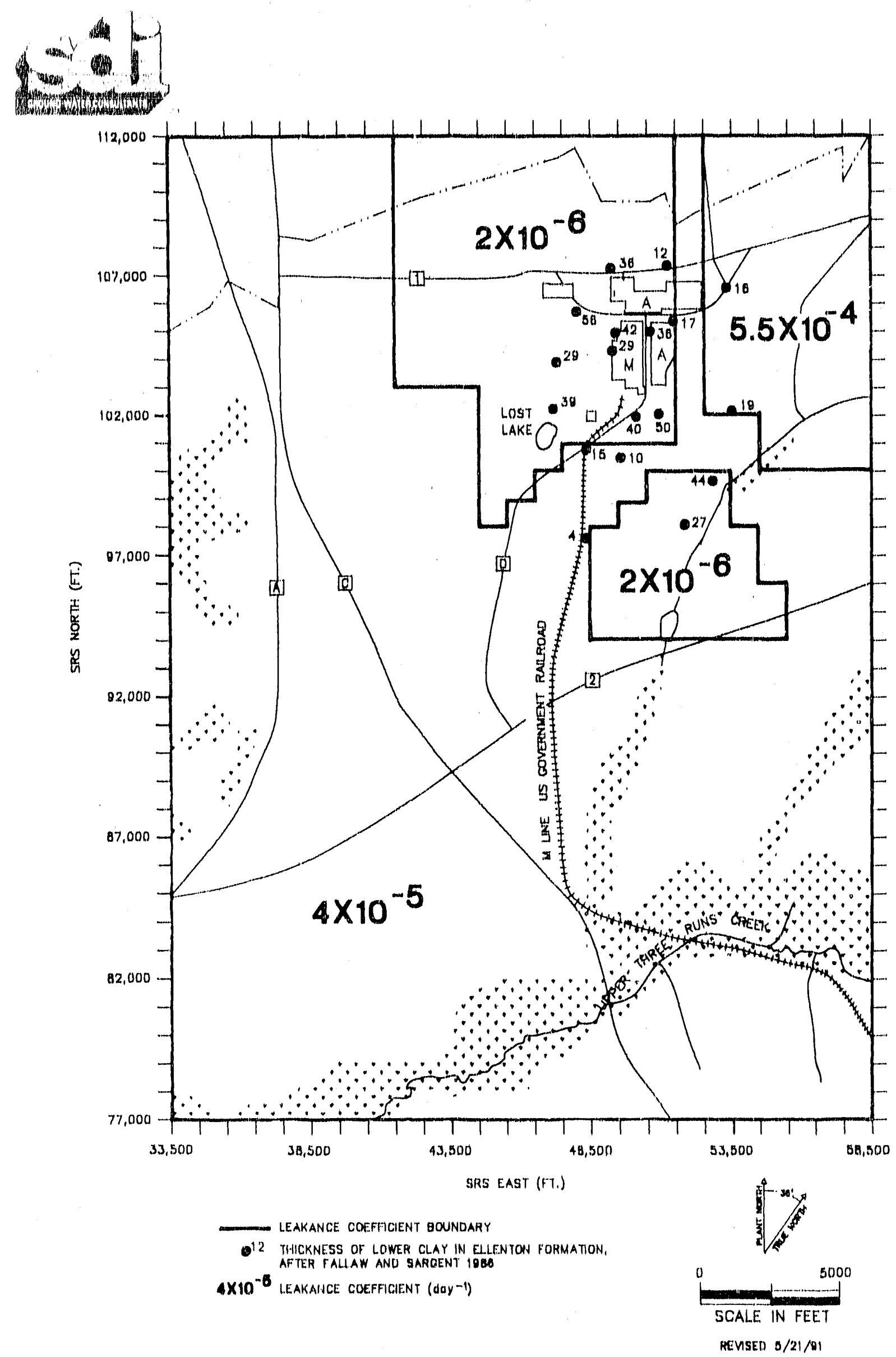

Figure 21. Calibrated Leakance Map for Model Layer 3.

A Ground Water flow Model for the A/M Area of the SRS (U)

Page 48 


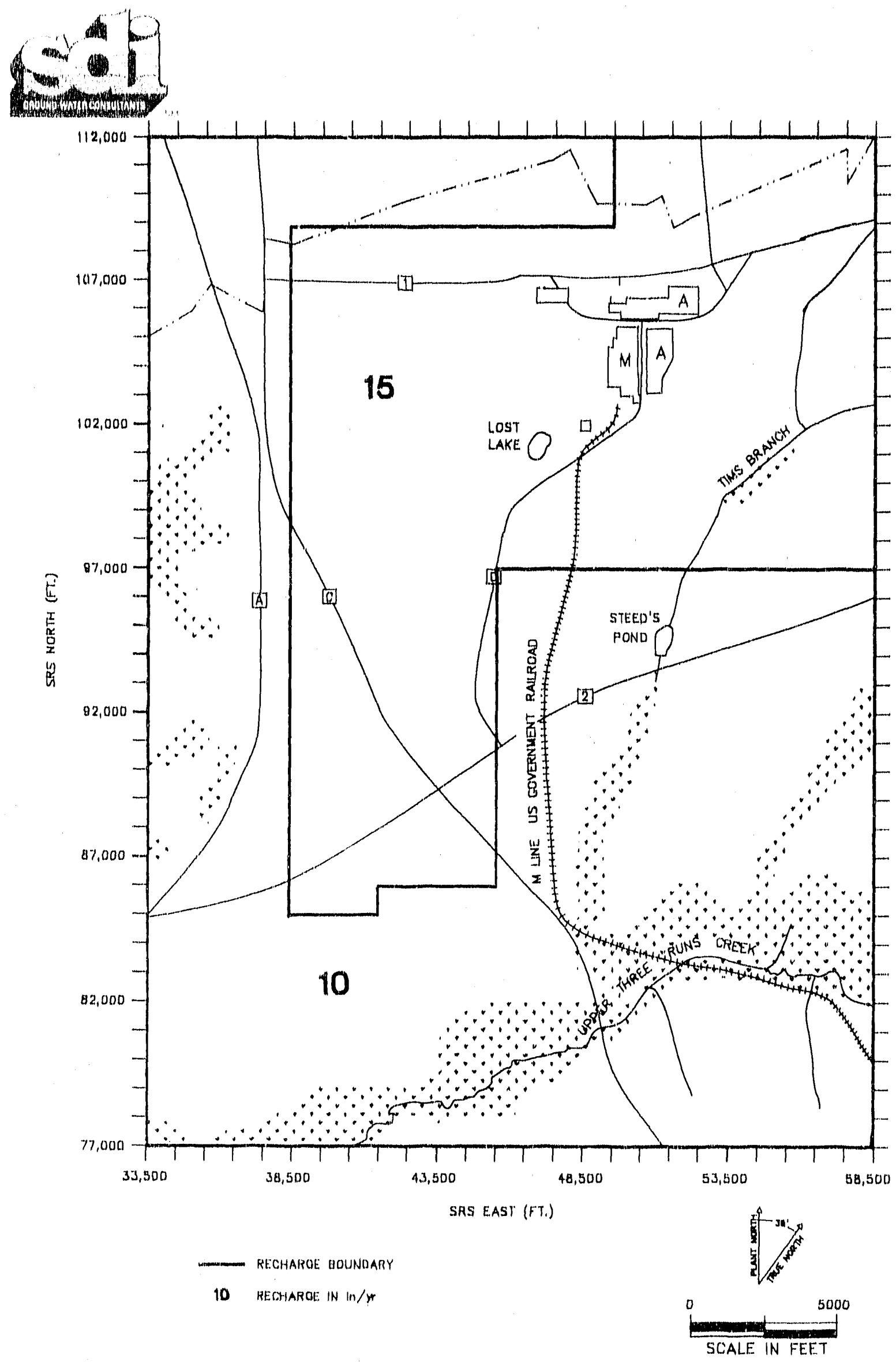

Figure 22. Modeled Rainfall Recharge.

A Ground Water Flow Model for the A/M Area of the SRS (U) 

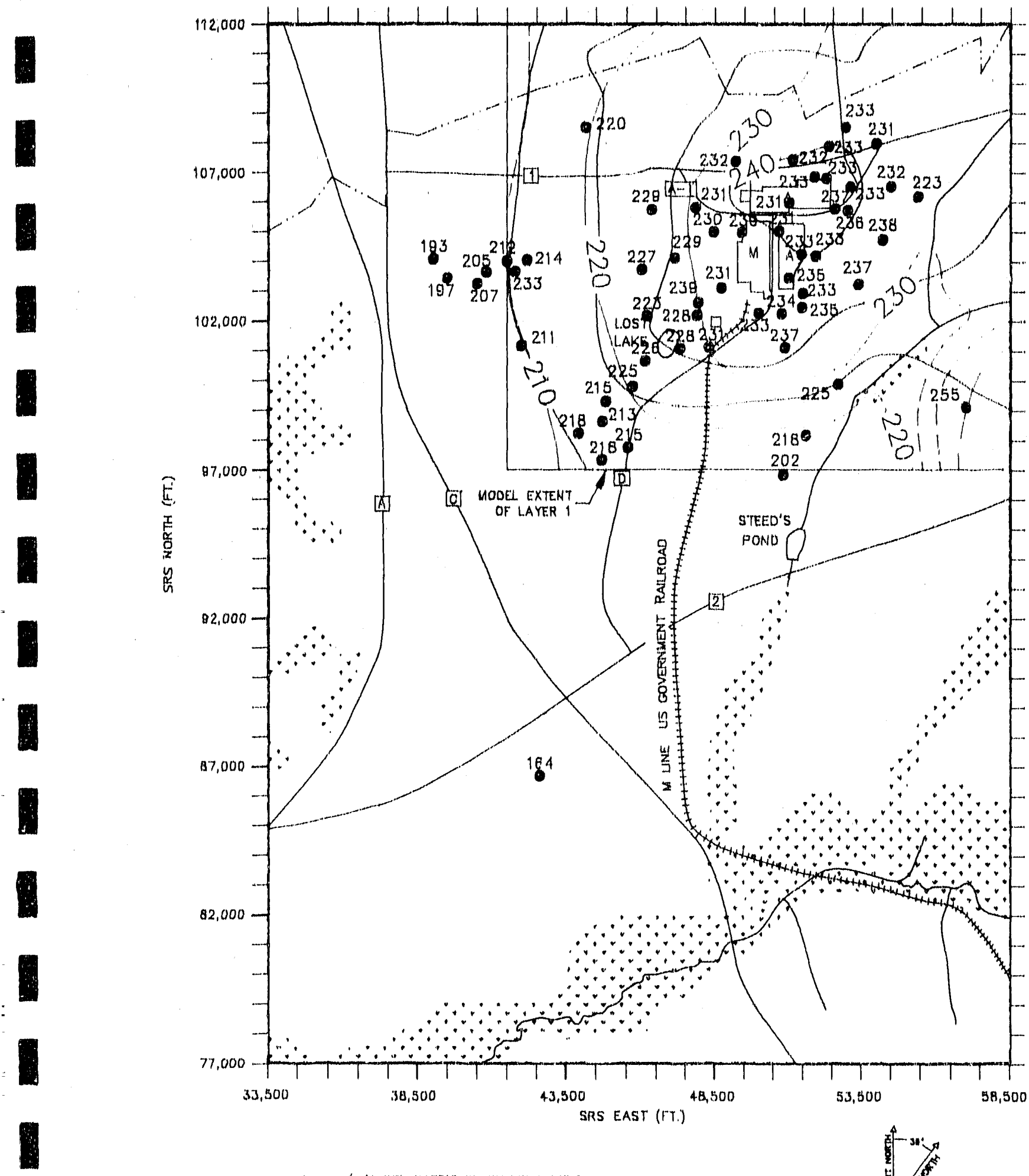

1
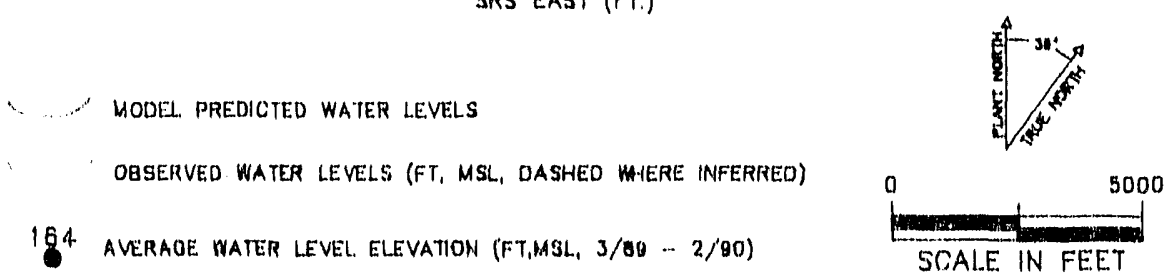

Figure 23. Observed and Model-Predicted Water Levels in Layer 1.

A Ground Water Flow Model for the A/M Area of the SRS (U) 


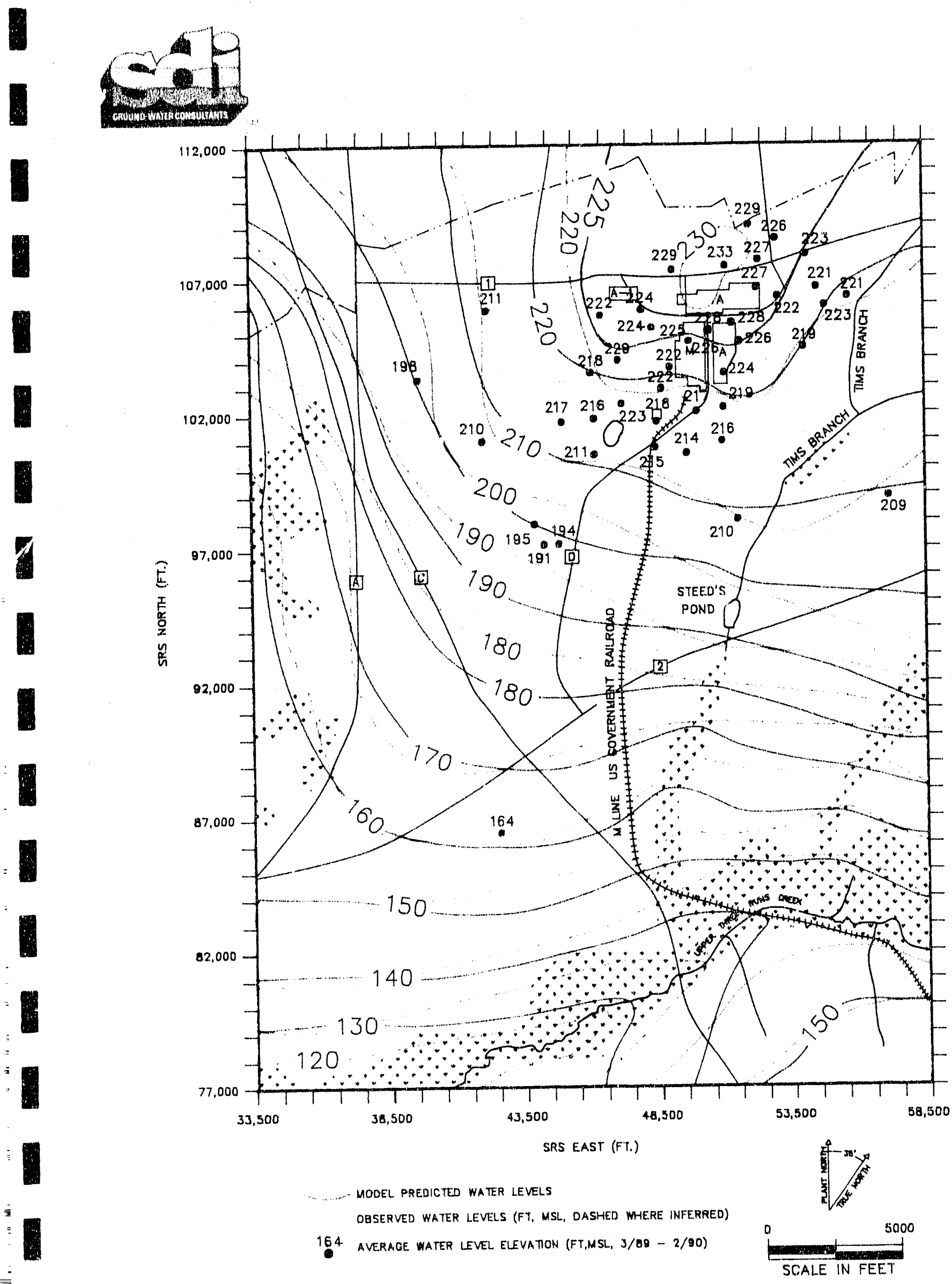

Figure 24. Observed and Model-Predicted Water Levels in Layer 2.

A Ground Water Flow Model for the A/M Area of the SRS (U) 


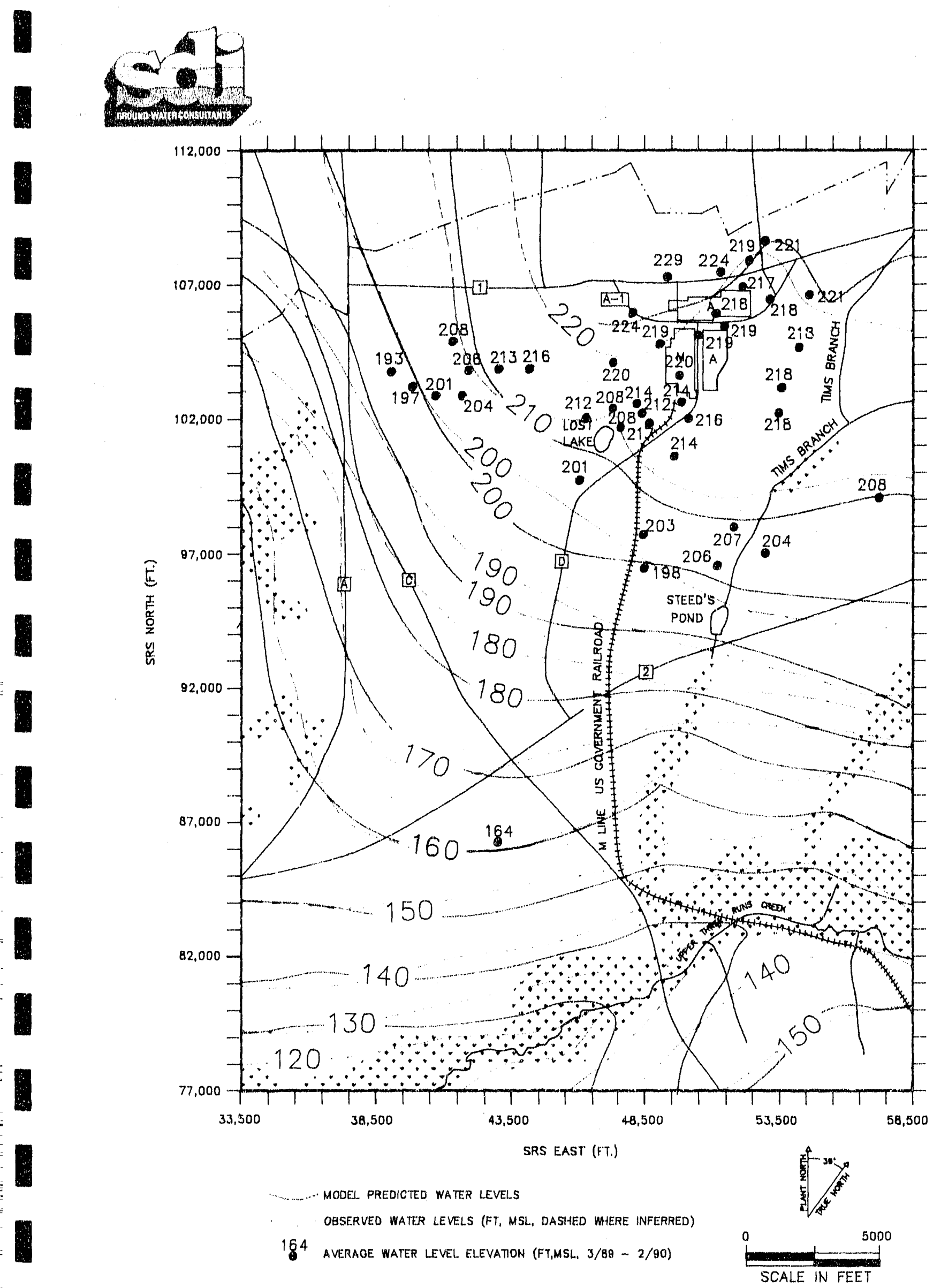

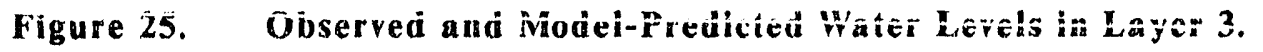

A Ground Water Flow Model for the A/M Area of the SRS (U) 


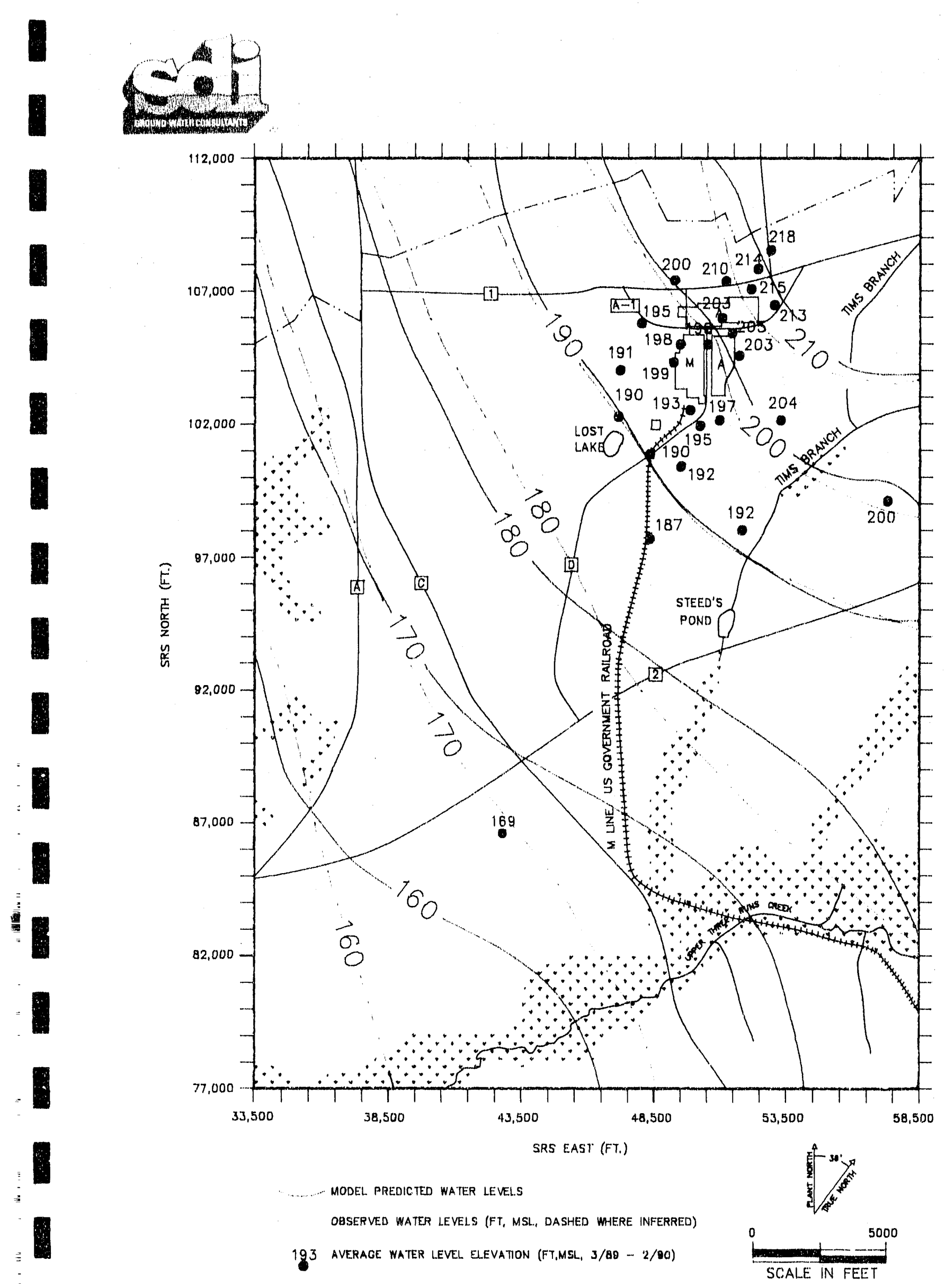

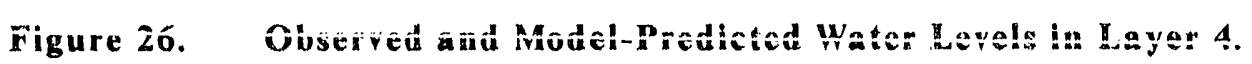




\section{APPENDIX A}

Water Level Data 


\section{APPENDIX A}

Table A-1. Water Table Observed Water Level Data.

\begin{tabular}{|c|c|c|c|}
\hline Well No. & $\begin{array}{c}\text { North SRP } \\
\text { (fi) }\end{array}$ & $\begin{array}{c}\text { East SRP } \\
\text { (ft) }\end{array}$ & $\begin{array}{c}\text { Water Level* } \\
\text { (ft, msl). }\end{array}$ \\
\hline $\mathrm{ABP} \backslash \mathrm{A}$ & 97501 & 44425 & 219.02 \\
\hline$A B P$ IDD & 97511 & 44434 & 219.36 \\
\hline $\mathrm{ABP} 2 \mathrm{~A}$ & 97764 & 44119 & 217.47 \\
\hline$A B P$ 2DD & 97754 & 44127 & 217.11 \\
\hline ABP 3 & 97794 & 44509 & 219.93 \\
\hline ABP 4 & 97481 & 44096 & 216.74 \\
\hline ABP 4DD & 97496 & 44101 & 217.59 \\
\hline ABP $6 \mathrm{D}$ & 97890 & 44101 & 217.34 \\
\hline$A B P 7 D$ & 97450 & 43930 & 217.65 \\
\hline$A B P$ 8D & 97855 & 43984 & 217.08 \\
\hline $\mathrm{AC} 2 \mathrm{~B}$ & 105649 & 46445 & 228.71 \\
\hline AC $3 \mathrm{~B}$ & 100997 & 42114 & 211.35 \\
\hline$A C B \perp A$ & 102624 & 51360 & 235.88 \\
\hline $\mathrm{ACB} 2 \mathrm{~A}$ & 102349 & 51559 & 236.77 \\
\hline $\mathrm{ACB} 3 \mathrm{~A}$ & 102162 & 51332 & 236.85 \\
\hline $\mathrm{ACB} 4 \mathrm{~A}$ & 102324 & 51113 & 237.50 \\
\hline AMB 4 & 104126 & 51480 & 232.95 \\
\hline AMB 5 & 104083 & 51467 & 232.87 \\
\hline AMB 6 & 104034 & 51466 & 232.93 \\
\hline $\mathrm{AMB} 7$ & 103920 & 51625 & 233.24 \\
\hline AOB 1 & 101918 & 50486 & 235.12 \\
\hline $\mathrm{AOB} 2$ & 102010 & 50725 & 235.35 \\
\hline ARP 2 & 99120 & 44876 & 214.99 \\
\hline ARP 3 & 98638 & 44904 & 216.66 \\
\hline ARP 4 & 98568 & 44375 & 213.13 \\
\hline ASB $2 A$ & 105615 & 52855 & 237.07 \\
\hline ASB $3 \mathrm{~A}$ & 105664 & 53150 & 237.43 \\
\hline ASB 4 & 105942 & 53175 & 236.38 \\
\hline ASB $5 A$ & 105892 & 52863 & 236.17 \\
\hline $\mathrm{ASB} 6 \mathrm{~A}$ & 105722 & 52674 & 235.46 \\
\hline ASB 7 & 105777 & 52625 & 234.04 \\
\hline ASB 8 & 106390 & 53132 & 232.71 \\
\hline ASB 9 & 104589 & 54226 & 238.39 \\
\hline MCB 2 & 97017 & 45129 & 218.40 \\
\hline MCB 4 & 97532 & 44705 & 219.82 \\
\hline MCB 5 & 97336 & 44864 & 219.65 \\
\hline MCB 6 & 97426 & 45214 & 215.25 \\
\hline MCB \&D & 97181 & 44770 & 220.04 \\
\hline
\end{tabular}

A Ground Water Flow Model for the A/M Area of the SRS (U)

Page A-1 
Table A-1. Water Table Observed Water Level Data (Continued).

\begin{tabular}{lccc} 
Well NO. & $\begin{array}{c}\text { North SRP } \\
(\mathbf{f l})\end{array}$ & $\begin{array}{c}\text { East SRP } \\
\text { (ft) }\end{array}$ & $\begin{array}{c}\text { Water Level* } \\
\text { (ft. msl) }\end{array}$ \\
\cline { 2 - 2 } 9D & 97606 & 44859 & 217.91 \\
MSB 6A & 101105 & 46328 & 224.80 \\
MSB 7A & 100564 & 46737 & 226.17 \\
MSB 8A & 100796 & 47303 & 227.72 \\
MSB 9B & 102466 & 47951 & 229.77 \\
MSB 10C & 102466 & 47951 & 229.34 \\
MSB 11D & 102670 & 48580 & 230.02 \\
MSB 13C & 101746 & 47522 & 228.38 \\
MSB 15D & 102971 & 48827 & 231.14 \\
MSB 17B & 101995 & 46238 & 225.20 \\
MSB 17D & 102057 & 46226 & 226.76 \\
MSB 18B & 100431 & 46121 & 220.01 \\
MSB 18C & 100431 & 46121 & 225.62 \\
MSB 19C & 100992 & 50942 & 236.94 \\
MSB 20C & 103556 & 46089 & 226.51 \\
MSB 21C & 103973 & 47237 & 229.38 \\
MSB 24 & 104614 & 49843 & 237.03 \\
MSB 27 & 104973 & 49488 & 239.05 \\
MSB 28 & 104942 & 48517 & 230.40 \\
MSB 29D & 107323 & 51227 & 232.26 \\
MSB 29DD & 107311 & 51191 & 232.37 \\
MSB 30C & 105731 & 48014 & 230.65 \\
MSB 31C & 101980 & 50090 & 233.79 \\
MSB 32 & 99656 & 52734 & 225.10 \\
MSB 33 & 98031 & 51736 & 217.75 \\
MSB 34C & 104934 & 50536 & 230.57 \\
MSB 39D & 100859 & 48396 & 231.23 \\
MSB 43D & 107274 & 49322 & 231.44 \\
MSB 43DD & 107273 & 49341 & 231.63 \\
MSB 44C & 103296 & 51107 & 235.11 \\
MSB 47D & 106960 & 52184 & 233.25 \\
MSB 48D & 107914 & 54056 & 231.41 \\
MSB 49D & 99725 & 45856 & 224.94 \\
MSB 50D & 96617 & 51044 & 202.02 \\
MSB 51D & 97016 & 52816 & 209.05 \\
MSB 52D & 103063 & 53417 & 236.52 \\
MSB 53D & 106448 & 54553 & 231.61 \\
MSB 54D & 108462 & 52985 & 232.69 \\
MSB 56D & 108464 & 44208 & 220.31 \\
MSB 61D & 106095 & 55391 & 223.32 \\
& & &
\end{tabular}

A Ground Water Flow Model for the A/M Area of the SRS (U)

Page A-2 
Table A-1. Water Table Observed Water Level Data (Continued).

$\begin{array}{lccc}\text { Well No. } & \begin{array}{c}\text { North SRP } \\ \text { (fil) }\end{array} & \begin{array}{c}\text { East SRP } \\ \text { (ft) }\end{array} & \begin{array}{c}\text { Water Level* } \\ \text { (ftemsl) }\end{array} \\ \text { MSB 65D } & 101916 & 49414 & 233.08 \\ \text { MSB 66D } & 105842 & 51044 & 231.23 \\ \text { MSB 67D } & 106831 & 51972 & 232.86 \\ \text { MSB 68D } & 106741 & 52294 & 233.44 \\ \text { MSB 69D } & 107784 & 52462 & 232.78 \\ \text { SRW 2 } & 103722 & 41627 & 212.23 \\ \text { SRW 3A } & 103516 & 41851 & 211.05 \\ \text { SRW 4 } & 103360 & 41612 & 211.83 \\ \text { SRW 5 } & 103418 & 41240 & 209.56 \\ \text { SRW 6 } & 103603 & 41244 & 210.10 \\ \text { SRW 7 } & 103541 & 40926 & 208.71 \\ \text { SRW 8 } & 103470 & 40456 & 206.48 \\ \text { SRW 9 } & 103260 & 39688 & 196.65 \\ \text { SRW 11 } & 103693 & 40874 & 208.49 \\ \text { SRW 12C } & 103712 & 39023 & 193.33 \\ \text { SRW 13C } & 102987 & 40682 & 207.28 \\ \text { SRW 15C } & 104775 & 41245 & 210.81 \\ \text { SRW 16C } & 103772 & 42842 & 213.71\end{array}$

* Average of Quarters II, III, \& IV (1989), and Quarter I (1990). 
Table A-2. Upper Congareo Observed Water Level Data.

\begin{tabular}{lccc} 
Well NQ & $\begin{array}{c}\text { North SRP } \\
\text { (ft) }\end{array}$ & $\begin{array}{c}\text { East SRP } \\
\text { (fi) }\end{array}$ & $\begin{array}{c}\text { Water Level* } \\
\text { (ft dusl) }\end{array}$ \\
\hline ABP 3C & 97778 & 44506 & 194.04 \\
ABP 8C & 97856 & 44059 & 194.73 \\
ABW 1 & 105954 & 55013 & 223.15 \\
AC 1A & 105865 & 42239 & 211.46 \\
AC 2A & 1056.36 & 46428 & 221.97 \\
AC 3A & 100989 & 42120 & 209.74 \\
ASB 8C & 106354 & 53101 & 221.99 \\
ASB 9C & 104568 & 54201 & 218.65 \\
MCB 5C & 97315 & 44863 & 195.05 \\
MCB 6C & 97413 & 45207 & 195.16 \\
MCB 7C & 97140 & 44871 & 191.21 \\
MSB 11B & 102649 & 48579 & 219.94 \\
MSB 11C & 102649 & 48579 & 221.81 \\
MSB 12B & 102252 & 47140 & 217.29 \\
MSB 12C & 102252 & 47140 & 222.76 \\
MSB 13B & 101736 & 47524 & 196.69 \\
MSB 14B & 101639 & 48519 & 217.82 \\
MSB 15A & 102984 & 48827 & 221.62 \\
MSB 16A & 103694 & 48965 & 222.19 \\
MSB 17A & 101977 & 46246 & 215.59 \\
MSB 18A & 100416 & 46110 & 210.70 \\
MSB 19B & 100999 & 50935 & 216.10 \\
MSB 20A & 103545 & 46061 & 217.94 \\
MSB 21A & 103967 & 472.17 & 220.61 \\
MSB 23B & 104337 & 49287 & 223.79 \\
MSB 24A & 104685 & 49845 & 226.22 \\
MSB 26A & 104602 & 48941 & 224.72 \\
MSB 27B & 104940 & 49486 & 225.81 \\
MSB 28A & 104948 & 48522 & 223.83 \\
MSB 29C & 107315 & 51207 & 232.72 \\
MSB 30CC & 105724 & 47993 & 224.43 \\
MSB 31CC & 101983 & 50068 & 215.61 \\
MSS 33C & 97985 & 51747 & 209.98 \\
MSB 34B & 104945 & 50535 & 227.78 \\
MSB 35B & 102111 & 50948 & 219.14 \\
MSB 36C & 100518 & 49537 & 213.58 \\
MSB 37C & 105283 & 51440 & 228.18 \\
MSB 38C & 102373 & 4976.2 & 216.86 \\
MSB 39C & 100852 & 48387 & 214.58 \\
MSB 42B & 104570 & 51583 & 225.63 \\
& & &
\end{tabular}

A Ground Water Flow Model for the A/M Area of the SRS (U) 
Table A-2. Upper Congaroo Observod Water Lovel Data (Continued),

$\begin{array}{lccc}\text { Well No. } & \begin{array}{c}\text { North SRP } \\ \text { (fi) }\end{array} & \begin{array}{c}\text { East SRP } \\ \text { (fi) }\end{array} & \begin{array}{c}\text { Wator Levol* } \\ \text { (fi. msl) }\end{array} \\ \text { MSB 42C } & 104582 & 51583 & 231.03 \\ \text { MSB 43B } & 107275 & 49312 & 229.43 \\ \text { MSB 44B } & 103296 & 51096 & 224.30 \\ \text { MSB 45B } & 103988 & 50555 & 225.94 \\ \text { MSB 47B } & 106975 & 52207 & 226.67 \\ \text { MSB 47C } & 106975 & 52207 & 232.27 \\ \text { MSB 48C } & 107917 & 54076 & 223.10 \\ \text { MSB 53C } & 106456 & 54541 & 221.39 \\ \text { MSB 54C } & 108447 & 52956 & 225.53 \\ \text { MSB 55C } & 108326 & 52030 & 228.74 \\ \text { MSB 61C } & 106091 & 55407 & 221.39 \\ \text { MSB 66C } & 103967 & 47217 & 228.61 \\ \text { MSB 67C } & 106731 & 52305 & 227.39 \\ \text { MSH 68C } & 106731 & 52305 & 226.56 \\ \text { MSB 69C } & 107780 & 52448 & 22.7 .36 \\ \text { MSB 70C } & 101785 & 45012 & 216.79 \\ \text { MSB 82C } & 107522 & 51949 & 228.38 \\ \text { MSB 83C } & 108405 & 52385 & 227.38 \\ \text { MSB 84C } & 108968 & 51974 & 229.08 \\ \text { MSB 85C } & 107835 & 53151 & 223.92 \\ \text { SRW 9B } & 103242 & 39697 & 197.59 \\ \text { SRW 12B } & 103703 & 39020 & 187.50\end{array}$

* Avurage of Quarters II, III, \& IV (1989), and Quarter I (1990). 
Table A-3. Lower Congaree Obsorved Water Lovel Data.

\begin{tabular}{|c|c|c|c|}
\hline Well Noe & $\begin{array}{l}\text { North SRP } \\
\text { (fi) }\end{array}$ & $\begin{array}{c}\text { East SRP } \\
\text { (fi) }\end{array}$ & $\begin{array}{l}\text { Water Lovol" } \\
\text { (ft. msl) }\end{array}$ \\
\hline $\mathrm{ASB} 8 \mathrm{~B}$ & 106362 & 53110 & 218.33 \\
\hline ASB 9B & 104565 & 54215 & 218.13 \\
\hline MSB 9A & 102239 & 48252 & 211.98 \\
\hline MSB 10B & 102488 & 47943 & 213.60 \\
\hline $\mathrm{MSB} 12 \mathrm{~A}$ & 102252 & 47140 & 208.46 \\
\hline MSB I $13 \mathrm{~A}$ & 101727 & 47525 & 207.94 \\
\hline $\mathrm{MSB} 14 \mathrm{~A}$ & 101630 & 48522 & 216.99 \\
\hline MSB 17BB & 102009 & 46221 & .211 .61 \\
\hline $\mathrm{MSB} 21 \mathrm{~B}$ & 104000 & 47272 & 220.12 \\
\hline $\mathrm{MSB} 25 \mathrm{~A}$ & 103505 & 49658 & 220.32 \\
\hline MSB 26B & 104647 & 48945 & 219.48 \\
\hline MSB 29B & 107319 & 51218 & 223.51 \\
\hline MSB $30 \mathrm{~B}$ & 105720 & 47982 & 224.14 \\
\hline MSB $3 / B$ & 101981 & 50079 & 215.83 \\
\hline MSB $33 B$ & 97996 & 51742 & 207.37 \\
\hline $\mathrm{MSB} 34 \mathrm{~A}$ & 104955 & 50535 & 318.60 \\
\hline MSB $36 \mathrm{~B}$ & 100515 & 49526 & 213.53 \\
\hline MSB $37 \mathrm{~B}$ & 105290 & 51450 & 218.64 \\
\hline MSB 38B & 102361 & 49746 & 213.65 \\
\hline MSB 39B & 100845 & 48377 & 211.12 \\
\hline $\mathrm{MSB} 40 \mathrm{~B}$ & 97685 & 48282 & 202.56 \\
\hline MSB $4 \mid C$ & 102177 & 53411 & 216.09 \\
\hline $\mathrm{MSB} 42 \mathrm{~A}$ & 102204 & 53411 & 217.95 \\
\hline $\mathrm{MSB} 43 \mathrm{~A}$ & 107276 & 49294 & 229.08 \\
\hline MSB 49B & 99738 & 45868 & 201.24 \\
\hline MSB $50 \mathrm{~B}$ & 96433 & 51054 & 207.77 \\
\hline MSB 51B & 96993 & 52818 & 203.51 \\
\hline MSB 5.2B & 103078 & 53418 & 217.50 \\
\hline MSB 53B & 106444 & 54574 & 220.66 \\
\hline MSB 54B & 108447 & 52971 & 220.71 \\
\hline MSB 66B & 105842 & 51065 & 218.19 \\
\hline MSB 67B & 106842 & 51990 & 217.81 \\
\hline MSB 68B & 106842 & 51990 & 217.44 \\
\hline MSB 69B & 107776 & 52433 & 219.41 \\
\hline MSB $71 \mathrm{~B}$ & 103802 & 44055 & 216.46 \\
\hline MSB 72B & 96388 & 48350 & 198.26 \\
\hline MSB 73B & 107523 & 51949 & 200.12 \\
\hline SRW $2 B$ & 103730 & 41632 & 205.93 \\
\hline SRW $9 A$ & 103251 & 39693 & 196.98 \\
\hline SRW $12 \mathrm{~A}$ & 103710 & 39013 & 192.59 \\
\hline
\end{tabular}

A Ground Water Flow Model for the A/M Area of the SRS (U) 
Table A-3. Lower Congareo Observed Wator Levol Data (Continued),

$\begin{array}{lccc}\text { Well No. } & \begin{array}{c}\text { North SRP } \\ \text { (ft) }\end{array} & \begin{array}{c}\text { East SRP } \\ \text { (fi) }\end{array} & \begin{array}{c}\text { Water Level* } \\ \text { (flemsl) }\end{array} \\ \text { SRW 13B } & 102994 & 40676 & 201.48 \\ \text { SRW 14B } & 102836 & 41548 & 203.54 \\ \text { SRW 15B } & 104773 & 41252 & 207.95 \\ \text { SRW 16B } & 103772 & 42826 & 212.99\end{array}$

* Average of Quarters II, III, \& IV (1989), and Quartor I (1990). 
Table A.4. Black Creok Ohserved Water Level Data.

\begin{tabular}{|c|c|c|c|}
\hline Wall $N_{\Omega}$ & $\begin{array}{c}\text { North SRP } \\
\text { (ft) }\end{array}$ & $\begin{array}{c}\text { East SRP } \\
\text { _(fi) }\end{array}$ & $\begin{array}{l}\text { Wator lovel" } \\
\text { (flemsil) }\end{array}$ \\
\hline ASB 8TA & 106375 & 53125 & 213.24 \\
\hline MSB $12 \mathrm{TA}$ & 102.260 & 47133 & 189.87 \\
\hline MSB $12 \mathrm{~TB}$ & 102260 & 47133 & 190.26 \\
\hline MSB $21 \mathrm{~T}^{\prime} \mathrm{A}$ & 103980 & 47218 & 191.13 \\
\hline MSB $23 \mathrm{~T}^{\mathrm{A}} \mathrm{A}$ & 104299 & 49226 & 198.69 \\
\hline MSB $27 \mathrm{~T}^{\mathrm{A}}$ & $104 ! 5 !$ & 49487 & 197.61 \\
\hline MSB 29TA & 107330 & 51246 & 209.98 \\
\hline $\mathrm{MSB} 30 \mathrm{~A}$ & 105727 & 48004 & 195.35 \\
\hline $\mathrm{MSB} 3 \mid \mathrm{A}$ & 101979 & 50100 & 195.34 \\
\hline MSB $33 \mathrm{TA}$ & 98018 & 51734 & 192.39 \\
\hline MSB $34 \mathrm{TA}$ & 104892 & 50538 & 195.30 \\
\hline MSB $34 \mathrm{~TB}$ & 104892 & 50538 & 198.87 \\
\hline MSB $35 T^{\prime A}$ & 102102 & 50920 & 197.14 \\
\hline MSB 36TA & 100508 & 49503 & 192.13 \\
\hline MSB $37 \mathrm{~A}$ & 105295 & 51440 & 205.15 \\
\hline MSB $37 \mathrm{~T}^{\mathrm{A}}$ & 105295 & 51440 & 205.00 \\
\hline MSB $38^{\circ} \mathrm{TA}$ & 102435 & 49810 & 192.77 \\
\hline МSB $39^{\prime} \Gamma A$ & 100831 & 48358 & 190.01 \\
\hline MSB $40 \mathrm{TA}$ & 97660 & 48277 & 187.04 \\
\hline $\mathrm{MSB} 4 \mathrm{TT}^{\mathrm{T}} \mathrm{A}$ & 102177 & 53430 & 203.89 \\
\hline MSB 42TA & 104546 & 51583 & 203.46 \\
\hline $\mathrm{MSB} 43 \mathrm{TA}$ & 107276 & 49282 & 200.27 \\
\hline MSB 47TA & 106988 & 52219 & 214.74 \\
\hline $\mathrm{MSB} 54 \mathrm{TA}$ & 108446 & 52986 & 217.73 \\
\hline MSB $66 \mathrm{TA}$ & 105843 & 51097 & 202.90 \\
\hline MSB 69TA & 107772 & 52418 & 213.76 \\
\hline
\end{tabular}


Tablo A-5. Wells Not Usod In Contouring Observed Water Level Maps.

\begin{tabular}{|c|c|}
\hline Woll Ne. & Unlt \\
\hline$A C-1 B$ & Water Tablo \\
\hline AMB 10DD & Water Table \\
\hline$A R P \perp A$ & Water Tablo \\
\hline ASB $\mid A$ & Water Table \\
\hline MSE $\mid \mathrm{A}$ & Water Table \\
\hline MSB $2 A$ & Water Table \\
\hline MSB $3 A$ & Water Table \\
\hline MSB 4A. & Water Table \\
\hline MSB 5A & Wator Table \\
\hline MSB 9C & Water Table \\
\hline MSB 1OD & Water Table \\
\hline MSB $11 E$ & Water Table \\
\hline MSB $\| \mathrm{F}$ & Water Table \\
\hline MSB 12D & Water Table \\
\hline $\mathrm{MSB} 14 \mathrm{C}$ & Water Table \\
\hline MSB 15C & Water Table \\
\hline MSB 16C & Water Table \\
\hline MSB $17 \mathrm{C}$ & Water Table \\
\hline MSB 22 & Water Table \\
\hline MSB 23 & Water Table \\
\hline MSB 25 & Water Table \\
\hline MSB 26 & Water Table \\
\hline MSB 35D & Water Table \\
\hline MSB 36D & Water Table \\
\hline MSB 37D & Water Table \\
\hline MSB 38D & Water Table \\
\hline MSB 4OD & Water T'able \\
\hline MSB 41D & Water Table \\
\hline MSB 42D & Water Table \\
\hline MSB $45 \mathrm{C}$ & Water Table \\
\hline MSB 46C & Water Table \\
\hline MSB 51DD & Water Table \\
\hline MSB 55D & Water Table \\
\hline SRW 1 & Water Table \\
\hline SRW 10 & Water Table \\
\hline SRW IAC & Water Table \\
\hline MSB 27A & Upper Congarce \\
\hline $\mathrm{MSB} 40 \mathrm{C}$ & Upper Congaree \\
\hline MSB 46B & Upper Congaree \\
\hline
\end{tabular}




\section{APPENDIX B}

An Alternative Interpretation of the TCE Data in the Southern Sector of the Lower Congaree 


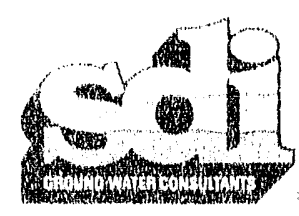

\section{APPENDIX B}

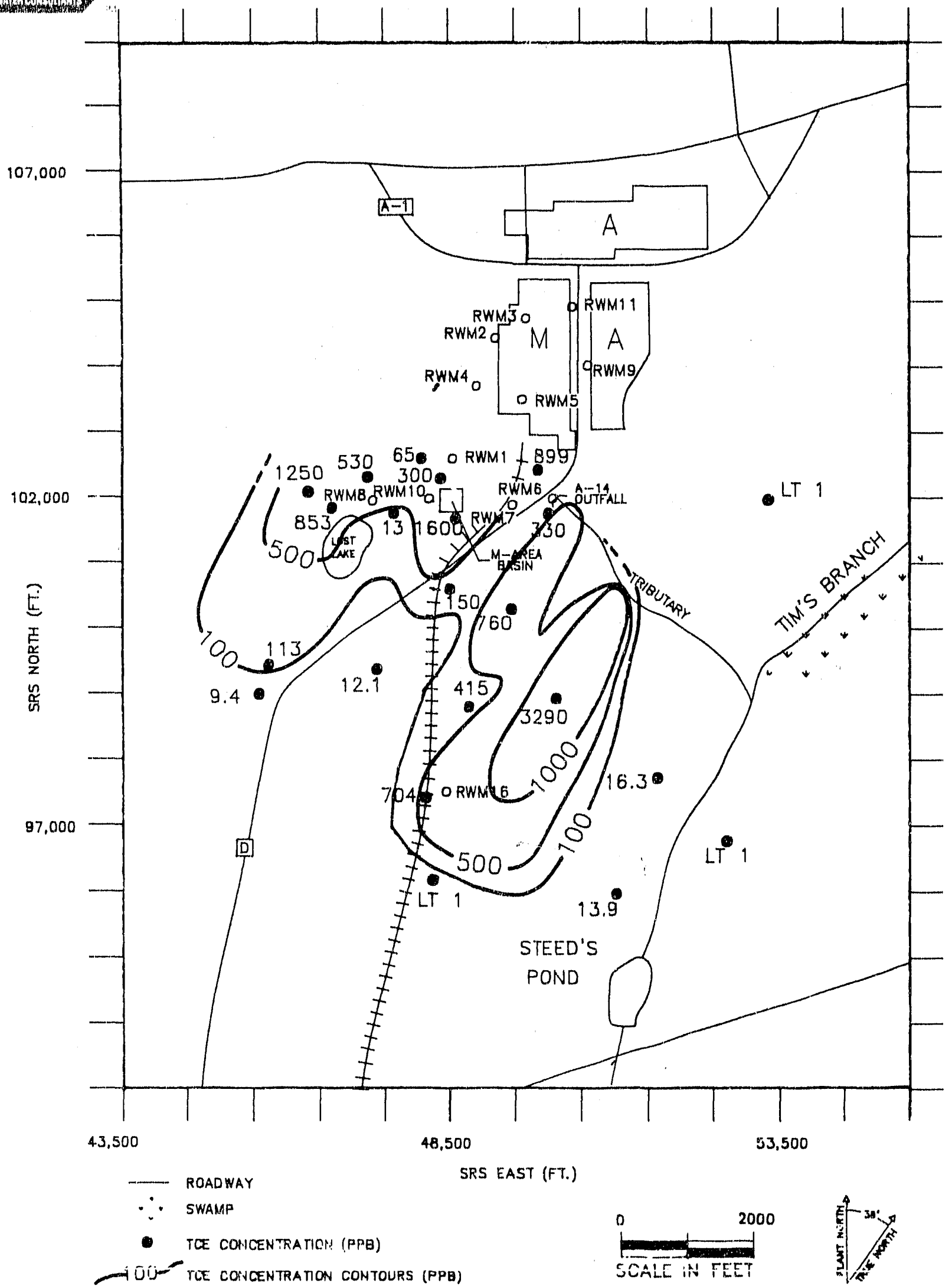

Figure B-1. An Alternative Interpretation of the TCE Data in the Southern Sector of the Lower Congaree. 


\section{APPENDIX C}

Hardcopy of Model Input

(Units in Feet and Days) 


\section{APPENDIX C1}

\section{Basic Package - Boundary Conditions}

and Starting Heads 
WSSC.BAS

COARSE GRID SIMULATION - WESTINGHOUSE SAVANAH RIVER COMPANY
GROUND WATER FLOW MODEL IN THE A/M AREA OF THE SRS
$\begin{array}{lllll}4 & 35 & 25 & 1 & 4\end{array}$

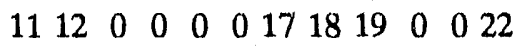

$0 \quad 0$

$61(40 I 2)$

00000000 0-1-1-1-1-1-1-1-1-1-1-1-1-1-1-1-1-1 $00000000-1111111111111111-1$ $00000000-1111111111111111-1$ $00000000-1111111111111111-1$ $00000000-11111111111111111$ $00000000-11111111111111111$ $00000000-11111111111111111$ $00000000-11111111111111111$ $00000000-11111111111111111$ $00000000-11111111111111111$ $00000000-11111111111111111$ $00000000-11111111111111111$ $00000000-11111111111011111$ $00000000-11111111111011111$ $00000000-1-1-1-1-1-1-1-1-1-10011111$ 0000000000000000000000000 0000000000000000000000000 0000000000000000000000000 0000000000000000000000000 0000000000000000000000000 0000000000000000000000000 0000000000000000000000000 0000000000000000000000000 0000000000000000000000000 0000000000000000000000000 0000000000000000000000000 0000000000000000000000000 0000000000000000000000000 0000000000000000000000000 0000000000000000000000000 0000000000000000000000000 0000000000000000000000000 0000000000000000000000000 0000000000000000000000000 0000000000000000000000000 6 1(4012) 2

1111111111 1-1-1-1-1-1-1-1-1-1-1-1-1-1-1 $111111111111111111111111-1$ 


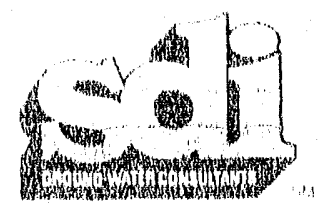

$111111111111111111111111-1$ $111111111111111111111111-1$ $111111111111111111111111-1$ $111111111111111111111111-1$ $111111111111111111111111-1$ $111111111111111111111111-1$ 1111111111111111111111114 $111111111111111111111111-1$ $1111111111111111111111111-1$ $111111111111111111111111-1$ $111111111111111111111111-1$ 1111111111111111111111111 1111111111111111111111111 1111111111111111111111111 1111111111111111111111111 11111111.11111111111111111 1111111111111111111111111 1111111111111111111111111 1111111111111111111111111 1111111111111111111111111 1111111111111111111111111 1111111111111111111111111 1111111111111111111111111 1111111111111111111111111 1111111111111111111111111 1111111111111111111111111. 1111111111111111111111111 1111111111111111111111111 1111111111111111111111111 1111111111111111111111111 1111111111111111111111111 $111111111111111111111111-1$ 11111111111111111 1-1-1-1-1-1-1-1

$$
6 \quad 1(40 ! 2)
$$
2

1111111111111 1-1-1-1-1-1-1-1-1-1-1-1-1 1111111111111111111111111 1111111111111111111111111 1111111111111111111111111 1111111111111111111111111 1111111111111111111111111 1111111111111111111111111 1111111111111111111111111 1111111111111111111111111 1111111111111111111111111 1111111111111111111111111 1111111111111111111111111 1111111111111111111111111

A Ground Water Flow Model for the A/M Area of the SRS (U) 
1111111111111111111111111 1111111111111111111111111 1111111111111111111111111 1111111111111111111111111 1111111111111111111111111 1111111111111111111111111 1111111111111111111111111 1111111111111111111111111 1111111111111111111111111 1111111111111111111111111 1111111111111111111111111 1111111111111111111111111 1111111111111111111111111 1111111111111111111111111 1111111111111111111111111 1111111111111111111111111 1111111111111111111111111 1111111111111111111111111 1111111111111111111111111 1111111111111111111111111 $111111111111111111111111-1$ 11111111111111111 1-1-1-1-1-1-1-1 6 $1(4012)$ 2

-111111111111 1-1-1-1-1-1-1-1-1-1-1-1-1 $-111111111111111111111111111-1$ $-111111111111111111111111-1$ $-111111111111111111111111-1$ $.111111111111111111111111-1$ $-111111111111111111111111-1$ $-111111111111111111111111-1$ $-111111111111111111111111-1$ $-111111111111111111111111-1$ $-111111111111111111111111-1$ $-111111111111111111111111-1$ $-111111111111111111111111-1$ $-111111111111111111111111-1$ $-111111111111111111111111-1$ $-111111111111111111111111-1$ $-111111111111111111111111-1$ $-111111111111111111111111-1$ $-111111111111111111111111-1$ $-111111111111111111111111-1$ $-111111111111111111111111-1$ $-111111111111111111111111-1$ $-111111111111111111111111-1$ $-111111111111111111111111-1$ $-111111111111111111111111-1$

A Ground Water Flow Model for the A/M Area of the SRS (U) 
$-111111111111111111111111-1$ $-111111111111111111111111-1$ $-111111111111111111111111-1$. $-111111111111111111111111-1$ $-111111111111111111111111-1$ $-111111111111111111111111-1$ $-111111111111111111111111-1$ $-111111111111111111111111.1$ $-111111111111111111111111-1$ $-111111111111111111111111-1$ $-111111111111111111111111-1$ 999.00

\begin{tabular}{|c|c|c|c|c|c|c|}
\hline \multicolumn{4}{|c|}{$60.100 \mathrm{E}+01(7 \mathrm{G} 11.4)$} & \multicolumn{2}{|c|}{12} & \multirow[b]{2}{*}{197.0} \\
\hline 173.5 & 174.2 & 176.8 & 181.0 & 186.0 & 191.4 & \\
\hline 202.1 & 207.4 & 211.5 & 214.5 & 216.3 & 220.0 & 221.2 \\
\hline 223.2 & 225.0 & 224.9 & 226.0 & 227.0 & 228.0 & 230.0 \\
\hline 231.0 & 232.0 & 233.0 & 234.0 & & & \\
\hline 170.5 & 171.6 & 174.6 & 179.9 & 185.6 & 191.5 & 197.0 \\
\hline 202.6 & 207.5 & 212.0 & 214.9 & 217.4 & 219.7 & 222.0 \\
\hline 224.2 & 225.8 & 226.2 & 227.4 & 228.3 & 232.0 & 235.8 \\
\hline 237.7 & 240.1 & 230.0 & 233.0 & & & \\
\hline 169.2 & 169.9 & 173.2 & 179.6 & 185.8 & 191.6 & 197.6 \\
\hline 203.2 & 207.6 & 212.3 & 232.0 & 219.3 & 221.0 & 223.2 \\
\hline 225.2 & 226.6 & 227.7 & $2,28.8$ & 229.7 & 232.2 & 235.0 \\
\hline 237.0 & 239.7 & 242.8 & 232.0 & & & \\
\hline 169.8 & 171.4 & 175.0 & 180.3 & 186.4 & 192.4 & 198.4 \\
\hline 203.6 & 208.0 & 213.0 & 217.1 & 220.5 & 222.4 & 224.6 \\
\hline 226.5 & 227.7 & 229.2 & 231.0 & 231.0 & 232.5 & 233.5 \\
\hline 235.4 & 239.3 & 243.6 & 231.0 & & & \\
\hline 171.4 & 173.7 & 177.3 & 182.4 & 187.8 & 193.5 & 199.2 \\
\hline 204.4 & 208.5 & 213.6 & 217.8 & 221.3 & 223.7 & 226.0 \\
\hline 228.0 & 229.6 & 230.6 & 231.4 & 232.1 & 232.7 & 231.9 \\
\hline 233.2 & 238.5 & 245.3 & 230.0 & & & \\
\hline 173.0 & 175.8 & 179.4 & 184.2 & 189.0 & 194.2 & 199.4 \\
\hline 204.8 & 209.1 & 214.1 & 218.0 & 221.7 & 224.6 & 227.2 \\
\hline 229.2 & 230.7 & 232.0 & 232.1 & 232.5 & 233.2 & 231.9 \\
\hline 231.7 & 237.1 & 250.1 & 233.2 & & & \\
\hline 174.9 & 177.7 & 181.4 & 185.7 & 189.9 & 194.5 & 199.6 \\
\hline 205.5 & 210.1 & 214.4 & 218.1 & 221.6 & 225.2 & 228.4 \\
\hline 229.9 & 231.7 & 233.7 & 232.2 & 231.9 & 233.7 & 234.9 \\
\hline 231.1 & 231.3 & 259.3 & 258.3 & & & \\
\hline 175.8 & 179.6 & 183.1 & 186.9 & 190.2 & 193.9 & 199.1 \\
\hline 206.0 & 211.4 & 214.3 & 2.17 .7 & 221.4 & 225.2 & 228.2 \\
\hline 2.29 .7 & 230.6 & 238.8 & 231.0 & 232.0 & 235.2 & 236.9 \\
\hline 237.0 & 244.1 & 255.3 & 257.4 & & & \\
\hline 177.1 & 180.4 & 185.2 & 188.5 & 190.5 & 192.6 & 197.4 \\
\hline 206.0 & 211.6 & 213.4 & 216.7 & 220.8 & 224.7 & 227.8 \\
\hline
\end{tabular}

A Ground Water Flow Model for the A/M Area of the SRS (U) 


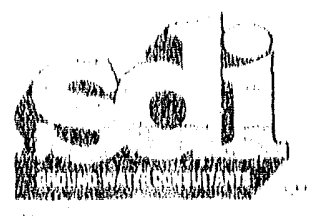

$\begin{array}{lllllll}229.7 & 231.7 & 234.9 & 234.0 & 233.0 & 235.2 & 237.2 \\ 240.2 & 246.4 & 252.3 & 254.0 & & & \\ 175.5 & 180.1 & 186.1 & 189.9 & 190.8 & 192.4 & 196.1 \\ 205.7 & 210.6 & 213.1 & 216.5 & 220.0 & 224.0 & 227.3 \\ 229.1 & 230.4 & 233.2 & 235.0 & 235.4 & 235.6 & 236.5 \\ 239.1 & 243.9 & 248.2 & 250.2 & & & \\ 172.4 & 178.7 & 183.3 & 187.9 & 189.9 & 192.7 & 197.6 \\ 203.8 & 209.1 & 213.0 & 216.3 & 219.0 & 223.0 & 226.6 \\ 228.5 & 230.8 & 233.1 & 235.1 & 236.6 & 235.0 & 234.6 \\ 236.2 & 239.0 & 2.43 .6 & 248.2 & & & \\ 170.9 & 172.4 & 176.6 & 182.4 & 186.9 & 191.5 & 196.1 \\ 202.2 & 207.9 & 212.5 & 215.2 & 217.8 & 221.7 & 225.3 \\ 228.4 & 231.4 & 232.7 & 235.2 & 235.1 & 232.1 & 230.7 \\ 232.3 & 237.6 & 244.0 & 248.9 & & & \\ 167.7 & 168.2 & 171.9 & 177.0 & 182.4 & 188.3 & 194.6 \\ 200.7 & 206.1 & 2.10 .7 & 213.2 & 216.2 & 221.9 & 224.8 \\ 226.6 & 228.0 & 229.0 & 230.2 & 229.6 & 227.4 & 224.8 \\ 229.1 & 237.4 & 246.5 & 251.7 & & & \\ 165.6 & 165.8 & 170.0 & 172.3 & 179.1 & 186.0 & 192.8 \\ 199.1 & 204.1 & 209.2 & 212.2 & 214.0 & 219.4 & 222.4 \\ 222.5 & 222.6 & 223.0 & 223.4 & 223.2 & 221.3 & 220.3 \\ 226.9 & 236.5 & 248.0 & 254.0 & & & \\ 165.0 & 16.5 .2 & 163.3 & 169.5 & 177.0 & 184.3 & 191.1 \\ 197.1 & 203.3 & 209.5 & 214.3 & 218.1 & 217.1 & 218.3 \\ 218.4 & 217.9 & 216.9 & 216.0 & 216.8 & 214.8 & 217.1 \\ 223.7 & 232.6 & 241.7 & 246.7 & & & \\ 164.8 & 170.0 & 168.0 & 171.1 & 177.0 & 183.6 & 190.3 \\ 195.9 & 202.3 & 207.8 & 213.9 & 218.4 & 216.1 & 213.6 \\ 212.2 & 211.3 & 209.7 & 207.2 & 206.6 & 208.0 & 212.6 \\ 219.2 & 226.5 & 233.3 & 238.0 & & & \\ 161.5 & 165.4 & 168.5 & 172.3 & 177.3 & 183.1 & 188.6 \\ 193.2 & 199.0 & 204.3 & 208.3 & 211.9 & 211.2 . & 209.5 \\ 207.5 & 205.5 & 203.9 & 201.8 & 201.8 & 204.5 & 208.5 \\ 213.8 & 219.8 & 225.4 & 229.7 & & & \\ 157.6 & 161.3 & 167.3 & 172.4 & 177.3 & 181.5 & 186.3 \\ 191.3 & 195.6 & 199.5 & 202.8 & 205.5 & 204.3 & 203.3 \\ 201.8 & 200.8 & 199.5 & 199.0 & 200.0 & 201.0 & 203.9 \\ 208.4 & 213.4 & 218.2 & 222.3 & & & \\ 154.1 & 160.1 & 167.0 & 172.1 & 176.4 & 180.6 & 184.8 \\ 188.8 & 191.7 & 194.7 & 196.8 & 198.7 & 197.7 & 197.4 \\ 196.7 & 195.4 & 195.0 & 195.0 & 195.0 & 195.6 & 198.5 \\ 201.9 & 206.2 & 210.8 & 214.8 & & & \\ 150.0 & 160.8 & 168.3 & 172.4 & 175.8 & 179.3 & 182.9 \\ 185.4 & 188.0 & 190.0 & 191.5 & 193.5 & 191.8 & 191.9 \\ 190.6 & 189.4 & 188.9 & 190.0 & 188.9 & 189.4 & 191.1 \\ 194.3 & 198.1 & 202.9 & 207.4 & & & \\ 154.6 & 163.8 & 170.0 & 172.0 & 174.8 & 177.7 & 180.7\end{array}$

A Ground Water Flow Model for the A/M Area of the SRS (U) 


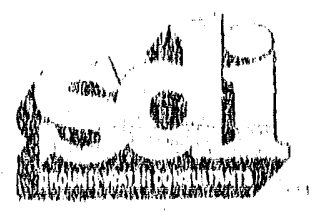

$\begin{array}{lllllll}182.4 & 184.3 & 185.6 & 186.3 & 188.2 & 185.6 & 185.7 \\ 184.0 & 182.4 & 181.3 & 180.0 & 181.1 & 182.0 & 183.9 \\ 185.6 & 188.5 & 193.1 & 199.4 & & & \\ 155.9 & 160.8 & 165.9 & 170.0 & 173.4 & 175.9 & 178.1 \\ 180.3 & 182.0 & 182.6 & 182.7 & 183.4 & 181.4 & 180.1 \\ 177.6 & 175.7 & 174.0 & 173.3 & 173.8 & 174.6 & 176.2 \\ 176.9 & 177.6 & 179.0 & 185.2 & & & \\ 156.5 & 159.8 & 164.7 & 169.4 & 172.1 & 173.8 & 176.1 \\ 177.3 & 177.9 & 178.4 & 177.7 & 177.5 & 176.4 & 174.3 \\ 171.7 & 169.1 & 167.0 & 166.8 & 167.3 & 168.7 & 168.9 \\ 168.4 & 168.1 & 168.3 & 173.5 & & & \\ 157.3 & 161.6 & 166.9 & 170.0 & 170.1 & 170.1 & 172.9 \\ 173.6 & 174.3 & 174.7 & 173.7 & 172.7 & 171.3 & 169.3 \\ 166.2 & 163.2 & 160.0 & 161.0 & 162.2 & 163.0 & 162.7 \\ 161.9 & 161.4 & 162.4 & 165.8 & & & \\ 157.4 & 161.9 & 164.7 & 166.5 & 166.9 & 167.0 & 167.2 \\ 167.8 & 170.3 & 170.3 & 169.8 & 168.4 & 166.0 & 164.4 \\ 161.8 & 159.3 & 157.5 & 157.4 & 157.8 & 158.0 & 157.7 \\ 156.1 & 155.2 & 156.2 & 158.9 & & & \\ 153.4 & 157.9 & 150.2 & 161.8 & 162.7 & 163.2 & 163.8 \\ 164.2 & 164.6 & 165.6 & 164.5 & 162.4 & 160.2 & 158.6 \\ 157.3 & 155.9 & 154.5 & 153.6 & 153.5 & 154.2 & 153.8 \\ 151.4 & 149.4 & 149.9 & 152.3 & & & \\ 148.2 & 151.9 & 154.8 & 156.5 & 157.4 & 158.4 & 159.0 \\ 159.5 & 160.0 & 160.8 & 159.4 & 157.3 & 155.7 & 154.4 \\ 153.4 & 152.4 & 152.0 & 149.5 & 147.6 & 150.0 & 149.9 \\ 148.6 & 144.5 & 143.7 & 145.8 & & & \\ 144.7 & 147.1 & 149.6 & 151.0 & 152.4 & 153.1 & 152.9 \\ 153.2 & 153.9 & 153.8 & 152.7 & 151.0 & 149.9 & 148.0 \\ 147.5 & 148.2 & 150.0 & 144.2 & 139.7 & 137.3 & 141.2 \\ 141.3 & 138.1 & 137.8 & 139.4 & & & \\ 140.9 & 142.6 & 144.3 & 145.5 & 146.8 & 146.6 & 147.0 \\ 147.1 & 147.4 & 147.0 & 145.8 & 144.3 & 142.4 & 141.6 \\ 141.5 & 141.6 & 140.8 & 137.3 & 133.3 & 131.9 & 131.8 \\ 131.8 & 132.2 & 134.8 & 137.4 & & & \\ 137.4 & 138.0 & 138.9 & 139.9 & 140.5 & 140.7 & 141.3 \\ 141.2 & 141.1 & 140.3 & 138.9 & 136.8 & 135.8 & 135.2 \\ 135.3 & 135.2 & 134.8 & 133.7 & 130.0 & 133.5 & 130.0 \\ 132.6 & 134.3 & 140.0 & 145.8 & & & \\ 133.3 & 133.9 & 134.5 & 135.2 & 135.2 & 135.3 & 135.4 \\ 134.9 & 134.9 & 134.7 & 133.1 & 130.0 & 129.3 & 129.1 \\ 130.0 & 130.4 & 131.8 & 137.9 & 144.6 & 150.3 & 152.7 \\ 154.1 & 157.2 & 162.8 & 166.1 & & & \\ 129.7 & 129.9 & 130.3 & 130.6 & 130.3 & 129.6 & 129.5 \\ 129.4 & 129.5 & 129.2 & 130.0 & 126.3 & 124.4 & 124.0 \\ 124.2 & 123.1 & 130.0 & 148.5 & 165.1 & 176.6 & 182.1 \\ 183.5 & 185.3 & 189.4 & 189.7 & & & \end{array}$

A Ground Water Flow Model for the A/M Area of the SRS (U)

C1-6 


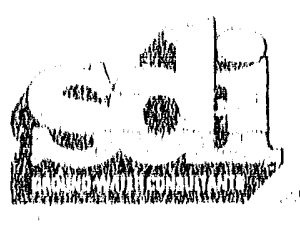

$\begin{array}{lllllll}126.1 & 125.7 & 126.0 & 126.1 & 125.6 & 125.0 & 124.5 \\ 124.5 & 124.4 & 124.0 & 123.8 & 122.0 & 120.6 & 121.2 \\ 124.5 & 127.5 & 142.4 & 164.5 & 188.3 & 204.9 & 212.7 \\ 210.6 & 209.1 & 210.0 & 204.4 & & & \\ 122.9 & 122.5 & 122.5 & 123.0 & 122.4 & 121.4 & 120.0 \\ 120.9 & 121.0 & 120.0 & 120.2 & 119.3 & 118.7 & 120.0 \\ 131.4 & 142.1 & 158.4 & 180.1 & 203.2 & 220.7 & 230.0 \\ 228.7 & 225.2 & 218.6 & 212.6 & & & \\ 120.0 & 120.0 & 120.7 & 120.7 & 120.3 & 119.7 & 119.3 \\ 119.8 & 119.8 & 119.8 & 120.0 & 119.9 & 120.0 & 130.0 \\ 140.1 & 154.2 & 171.4 & 192.2 & 213.2 & 228.6 & 236.3 \\ 238.2 & 237.7 & 226.9 & 219.6 & & & \\ 6 & 0.100 \mathrm{E}+01(7 \mathrm{G} 11.4) & & & 12 & & \\ 197.8 & 200.3 & 202.1 & 203.6 & 205.4 & 207.7 & 208.8 \\ 211.0 & 213.1 & 216.8 & 218.9 & 221.1 & 222.5 & 223.5 \\ 227.9 & 228.0 & 228.2 & 228.4 & 227.7 & 227.1 & 226.1 \\ 225.2 & 224.4 & 223.8 & 223.4 & & & \\ 196.6 & 199.0 & 201.1 & 203.1 & 204.8 & 207.3 & 208.6 \\ 211.1 & 212.6 & 216.1 & 218.3 & 220.9 & 222.5 & 223.8 \\ 227.8 & 228.4 & 228.8 & 229.0 & 228.1 & 227.0 & 225.7 \\ 224.6 & 223.9 & 223.3 & 222.8 & & & \\ 195.9 & 197.9 & 200.6 & 202.1 & 203.9 & 206.2 & 208.1 \\ 210.4 & 212.7 & 215.5 & 218.0 & 220.6 & 222.4 & 224.2 \\ 227.8 & 228.8 & 229.9 & 229.8 & 228.7 & 226.6 & 224.9 \\ 223.9 & 223.2 & 222.7 & 222.3 & & & \\ 193.9 & 196.0 & 199.3 & 201.4 & 203.1 & 205.2 & 207.3 \\ 209.7 & 212.1 & 215.6 & 217.7 & 219.9 & 222.0 & 224.3 \\ 227.5 & 229.1 & 230.5 & 230.7 & 228.8 & 225.5 & 223.8 \\ 223.0 & 222.5 & 222.0 & 221.7 & & & \\ 191.8 & 194.7 & 196.9 & 200.0 & 202.2 & 204.1 & 206.8 \\ 209.0 & 211.6 & 214.7 & 217.2 & 219.6 & 221.6 & 223.9 \\ 226.8 & 228.9 & 230.8 & 232.2 & 228.5 & 224.7 & 222.6 \\ 222.1 & 221.8 & 221.4 & 219.5 & & & \\ 190.2 & 193.2 & 195.6 & 197.9 & 200.4 & 202.6 & 205.4 \\ 208.1 & 211.0 & 214.0 & 216.5 & 218.9 & 221.3 & 223.6 \\ 225.6 & 227.6 & 229.6 & 230.5 & 227.9 & 222.8 & 221.6 \\ 221.9 & 221.2 & 220.9 & 218.6 & & & \\ 188.4 & 191.2 & 194.2 & 196.5 & 198.8 & 201.2 & 203.9 \\ 207.2 & 210.3 & 213.1 & 21.5 .8 & 218.2 & 220.8 & 223.1 \\ 224.2 & 225.8 & 227.8 & 228.7 & 226.8 & 222.8 & 220.9 \\ 221.6 & 220.5 & 218.5 & 217.5 & & & \\ 186.4 & 189.6 & 192.8 & 195.1 & 197.0 & 199.1 & 202.1 \\ 205.9 & 209.6 & 212.6 & 215.0 & 217.4 & 220.0 & 223.5 \\ 224.1 & 224.4 & 226.0 & 227.7 & 226.6 & 222.6 & 219.2 \\ 219.2 & 218.1 & 217.1 & 216.3 & & & \\ 183.5 & 187.5 & 191.2 & 193.4 & 195.0 & 196.7 & 199.7 \\ 204.8 & 209.1 & 212.2 & 215.0 & 216.6 & 217.8 & 22.2 .5\end{array}$




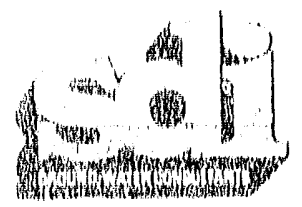

$\begin{array}{lllllll}223.2 & 222.0 & 222.8 & 224.9 & 224.0 & 221.9 & 219.9 \\ 218.0 & 216.9 & 216.0 & 215.2 & & & \\ 179.7 & 183.9 & 190.0 & 190.9 & 192.6 & 194.9 & 198.8 \\ 204.2 & 209.1 & 212.1 & 214.7 & 216.7 & 217.1 & 220.2 \\ 220.9 & 219.5 & 217.9 & 220.6 & 221.4 & 220.9 & 219.3 \\ 217.8 & 216.6 & 215.4 & 214.4 & & & \\ 174.6 & 177.7 & 182.5 & 186.3 & 189.4 & 193.1 & 197.6 \\ 203.5 & 209.2 & 211.4 & 213.3 & 215.3 & 214.7 & 216.2 \\ 217.2 & 216.3 & 215.1 & 217.4 & 219.3 & 219.8 & 218.9 \\ 217.2 & 215.5 & 214.1 & 213.1 & & & \\ 169.8 & 170.0 & 176.4 & 181.3 & 185.9 & 190.2 & 195.3 \\ 200.8 & 206.3 & 208.4 & 209.4 & 210.2 & 211.0 & 212.1 \\ 213.1 & 213.6 & 213.8 & 215.4 & 217.6 & 219.3 & 218.6 \\ 216.6 & 214.4 & 212.6 & 212.1 & & & \\ 1666.9 & 168.7 & 172.5 & 177.0 & 182.2 & 187.2 & 191.8 \\ 196.3 & 200.3 & 202.7 & 204.1 & 204.8 & 206.2 & 207.8 \\ 209.2 & 210.2 & 211.3 & 213.3 & 216.1 & 219.2 & 217.8 \\ 215.1 & 212.5 & 210.5 & 210.5 & & & \\ 165.3 & 163.6 & 166.0 & 173.3 & 179.0 & 184.1 & 188.5 \\ 192.0 & 195.1 & 196.9 & 198.3 & 199.3 & 201.5 & 203.7 \\ 2105.1 & 206.7 & 208.2 & 210.2 & 212.7 & 214.7 & 214.7 \\ 212.8 & 210.9 & 2099.2 & 209.6 & & & \\ 166.6 & 166.5 & 167.2 & 172.1 & 177.1 & 181.6 & 185.5 \\ 188.2 & 190.6 & 192.2 & 192.9 & 195.3 & 197.1 & 199.2 \\ 201.1 & 203.4 & 205.2 & 207.2 & 209.0 & 210.6 & 210.9 \\ 210.5 & 209.7 & 209.5 & 209.7 & & & \\ 166.0 & 168.3 & 169.5 & 172.6 & 176.2 & 179.8 & 183.1 \\ 185.7 & 187.2 & 188.0 & 188.5 & 190.7 & 193.2 & 195.4 \\ 197.5 & 199.9 & 202.0 & 204.2 & 205.7 & 206.9 & 207.7 \\ 208.2 & 208.6 & 209.3 & 210.0 & & & \\ 161.5 & 165.6 & 169.9 & 172.6 & 175.3 & 177.9 & 180.3 \\ 183.8 & 185.7 & 184.3 & 184.3 & 187.3 & 189.1 & 191.4 \\ 193.6 & 196.2 & 198.6 & 200.9 & 202.3 & 203.2 & 204.0 \\ 205.4 & 207.0 & 208.9 & 210.0 & & & \\ 157.5 & 162.7 & 168.4 & 171.8 & 174.4 & 176.8 & 178.6 \\ 180.3 & 181.1 & 181.0 & 181.6 & 184.2 & 185.6 & 187.1 \\ 189.4 & 192.1 & 195.1 & 197.1 & 198.0 & 198.7 & 199.6 \\ 202.2 & 204.8 & 207.8 & 209.9 & & & \\ 154.3 & 160.4 & 166.2 & 170.5 & 173.4 & 175.6 & 177.3 \\ 178.0 & 178.5 & 178.8 & 178.5 & 179.7 & 182.3 & 183.3 \\ 185.9 & 188.2 & 190.9 & 192.5 & 192.6 & 193.2 & 194.8 \\ 197.4 & 200.8 & 205.2 & 209.3 & & & \\ 153.4 & 159.6 & 165.5 & 170.0 & 172.8 & 174.6 & 175.6 \\ 176.6 & 176.3 & 177.1 & 176.7 & 177.6 & 178.8 & 179.8 \\ 181.5 & 182.4 & 184.2 & 185.4 & 185.7 & 186.7 & 188.1 \\ 190.4 & 194.3 & 200.1 & 204.9 & & & \\ 154.9 & 160.0 & 166.3 & 170.9 & 173.2 & 174.6 & 175.8\end{array}$

A Ground Water Flow Model for the A/M Area of the SRS (U) 


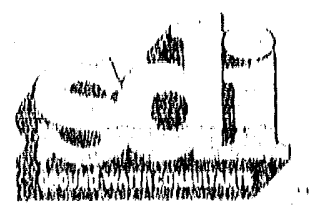

\begin{tabular}{|c|c|c|c|c|c|c|}
\hline 175.9 & 176.3 & 176.2 & 176.2 & 175,8 & 176,2 & 176.3 \\
\hline 175.8 & 176.2 & 176,4 & 177.3 & 178,6 & 179,5 & 180.4 \\
\hline 181.6 & 184.1 & $1(x), 3$ & 194.4 & & & \\
\hline 156.6 & 160.0 & 167.2 & 171.2 & 172,8 & 173.7 & 174.1 \\
\hline 174.9 & 174.7 & 175.3 & 173.4 & 172.8 & 172.2 & 171.7 \\
\hline 170.7 & 170.0 & 170.0 & 170,6 & 171,6 & 172.2 & 172.5 \\
\hline 172.6 & 172.3 & 175.2 & 181.6 & & & \\
\hline 158.8 & 163.5 & 168.9 & 170,9 & 171.7 & 172.1 & 172.5 \\
\hline 173.1 & 173,4 & 172.5 & 171.3 & 170.2 & 169.1 & 167.6 \\
\hline 165,7 & 163.8 & 163.5 & 164.8 & 165.6 & 16.5 .6 & 165,3 \\
\hline 164.8 & 164.8 & 167.2 & 171.7 & & & \\
\hline 160,0 & 164.2 & 168.1 & 169.0 & 169.3 & 169,6 & 170.0 \\
\hline 170.3 & $17(0.6$ & 169.8 & 168.6 & 166.2 & 165.2 & 163.4 \\
\hline 161.5 & 159.4 & 158.8 & 159.6 & 160,4 & 160.3 & 159.4 \\
\hline $1.58,5$ & 158.8 & 1.61 .0 & 164.1 & & & \\
\hline 160.0 & 161.7 & 16.3 .7 & 165.0 & 165.3 & 165.9 & 166.4 \\
\hline 167.0 & 167.5 & 167.0 & 165.4 & 163,0 & 161.4 & 1.59 .9 \\
\hline 158.0 & 156.6 & 155.7 & 155.5 & 156,1 & 155.9 & 1.54 .5 \\
\hline 1.52 .5 & 153.3 & 155.2 & 157.7 & & & \\
\hline 2.6 & 156.4 & 1.58 .6 & 159.8 & 160.5 & 161.2 & 161.7 \\
\hline 162.5 & 163.2 & 163.7 & 161.0 & 158,9 & 157.3 & 1.55 .9 \\
\hline 154.8 & 153.9 & 152.6 & 151.2 & 151.4 & 1.52 .1 & 151.4 \\
\hline 149.3 & 149.1 & 150.1 & 151.8 & & & \\
\hline 148.0 & 150.9 & 153.1 & 154.3 & $15 f_{j} .2$ & 156.0 & 156.5 \\
\hline 157.1 & 157.4 & 1.57 .0 & 155.2 & 153.4 & 152.3 & 151 \\
\hline 151.2 & 151.3 & 149.2 & 145.2 & 142,4 & 144.1 & 150,0 \\
\hline 146.6 & 14.5 .5 & 14.5 .5 & 146,0 & & & \\
\hline 144.0 & 146.0 & 147.5 & 148.7 & 149.7 & 150.2 & 150.8 \\
\hline 150.6 & 1.50 .6 & 150,0 & 148.5 & 147.1 & 146.4 & 146.2 \\
\hline 146,2 & 146.6 & 144.3 & 1.39 .4 & 137.0 & 137.7 & 140 \\
\hline 140.6 & 141.3 & 141.8 & 140.9 & & & \\
\hline 140.0 & 141.1 & 142.3 & 14.3 .3 & 143.9 & 144.3 & 144 \\
\hline 144.3 & 144.0 & 142,9 & 141.5 & 140.3 & 140,0 & 140 \\
\hline 140.3 & 140.2 & 138.6 & 136,0 & 133.8 & 132.7 & 132. \\
\hline 133.5 & 137.8 & 140.2 & 139.7 & & & \\
\hline 135.8 & 136,6 & 137.4 & 137.9 & 38.2 & 138.5 & 138. \\
\hline 138,2 & 137.9 & 136.8 & 1.34 .7 & 133.3 & 133.5 & 133. \\
\hline 134.4 & 134.7 & 134.3 & 132.8 & 131.1 & 129.6 & 120 \\
\hline 129.9 & 134.9 & 138.1 & 138.5 & & & \\
\hline 1.31 .8 & 132.2 & 132.6 & 132.9 & 132,9 & 132.8 & 132 \\
\hline 132,5 & 132,4 & 132,0 & 129.9 & 127.8 & 127.9 & 128. \\
\hline 128.6 & 129.6 & 131.2 & 130.4 & 129.3 & 128.4 & 128 \\
\hline 129.7 & 132.6 & 135,2 & 1.36 .5 & & & \\
\hline 127,9 & 128.2 & 128.4 & 128,3 & 128.0 & 127.5 & 127 \\
\hline 127.1 & 127.0 & 127.1 & 125.8 & 123.6 & 123.4 & 12.5 \\
\hline 123.7 & 123.6 & 128.1 & 128.4 & 1288,0 & 127.8 & $12 x$ \\
\hline 129.2 & 131.1 & 133.2 & 135.3 & & & \\
\hline
\end{tabular}

A Ground Water flow Model for the A/M Area of the SRS (U) 


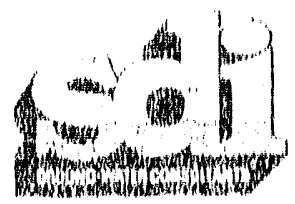

\begin{tabular}{|c|c|c|c|c|c|c|}
\hline 124,4 & 124.6 & 124.7 & 124.5 & 123.8 & 122.7 & 122,3 \\
\hline 122.7 & 122.3 & 122.1 & 121.6 & 120.6 & 1.21 .7 & 127.1 \\
\hline 12.7 .6 & 125.4 & 126.5 & 127.2 & 127,3 & 127.3 & 127.8 \\
\hline 128.8 & 130,2 & 132,0 & 133,6 & & & \\
\hline 12.1 .2 & 121.6 & 122,0 & 121.8 & 121.0 & 119,9 & 119.7 \\
\hline 120.2 & 119,8 & 119.5 & 119.5 & 119.7 & 124.2 & 129.2 \\
\hline 129.0 & 127,2 & 126.6 & 126.8 & 126.9 & 127,1 & 127.5 \\
\hline 128.2 & 129.5 & 1301.8 & 152,6 & & & \\
\hline 119.4 & 120.0 & 120.4 & 120.0 & 119.5 & 118.9 & 119,0 \\
\hline 119.6 & 119.6 & 119.1 & 119.2 & 120.1 & 124.7 & 128.8 \\
\hline 128.3 & 127.3 & 126.6 & 126.8 & 150.0 & 151.5 & 152.2 \\
\hline 154,8 & 1.55 .9 & 157.1 & 158.4 & & & \\
\hline \multicolumn{4}{|c|}{$6(0,100 \mathrm{E}+01(7011.4)$} & 12. & & \\
\hline 191.2 & 197.6 & 198.1 & 198.8 & 200.6 & 203.0 & 205.1 \\
\hline 211.1 & 213,0 & 215.0 & 220.1 & 220.2 & 225.3 & 2283.3 \\
\hline 228,3 & 227.4 & 226.8 & 226.1 & 2.25 .5 & 224.6 & 22.3 .9 \\
\hline 223.4 & 223.1 & 221.9 & 221.8 & & & \\
\hline 195.0 & 196,1 & 196.9 & 199,0 & 201.1 & 202.9 & 206.3 \\
\hline 210.4 & 212.6 & 214.7 & 220.2 & 221.6 & 221.9 & 228.4 \\
\hline 228.5 & 228.2 & 227.0 & 226.1 & 225.1 & 224.1 & 223.3 \\
\hline 222.9 & 221.8 & 221.7 & 221,6 & & & \\
\hline 193.3 & 194.3 & 196.4 & 198.2 & 2000.5 & 202.4 & 204,4 \\
\hline 207.8 & 212.0 & 214.4 & 219.7 & 221.2 & 222.6 & 228.2 \\
\hline 228.7 & 228.5 & 227.3 & 226.1 & 224.7 & 223.3 & 2.22 .5 \\
\hline 221.6 & 221.5 & 221.5 & 221.4 & & & \\
\hline 191.9 & 193.7 & 195.5 & 197.4 & 199.7 & 201.7 & 203.9 \\
\hline 207.4 & 210.3 & 212.7 & 2.17 .7 & 2.20 .9 & 222.4 & 226.5 \\
\hline 228.4 & 228.8 & 227.9 & 226.3 & 223.9 & 222.1 & 221.2 \\
\hline 221.2 & 221.2 & 221.2 & 221.1 & & & \\
\hline 191.0 & 192.7 & 194.4 & 196.4 & 198.6 & $2(0), 9$ & 203.4 \\
\hline 206.8 & 210.0 & 212.7 & 217.3 & 221.1 & 222.4 & 225.2 \\
\hline 228.1 & 228.8 & 228.4 & 226.0 & 222.9 & 220.0 & 220.2 \\
\hline 220.7 & 220,9 & $22(0.9$ & 220.8 & & & \\
\hline 189.9 & 191.6 & 193.5 & 195.3 & 197.4 & 199.7 & 202.5 \\
\hline 20.5 .4 & 209.7 & 212.7 & 215.3 & 220.3 & 22.2 .4 & 225.2 \\
\hline 226.9 & 227.6 & 227.4 & 224.2 & 220.5 & 218.0 & 219.2 \\
\hline 220.5 & 220.7 & 220.5 & 220.4 & & & \\
\hline 187.8 & $1(1) .5$ & 192.3 & 193.9 & 195.9 & 198.0 & 2.01 .2 \\
\hline 205.1 & $2(19.2$ & 212.5 & 216,3 & 219.0 & 220.9 & 223.4 \\
\hline 224.6 & 224.5 & 223.1 & 220,6 & 218.2 & 217.8 & 218.7 \\
\hline 219.9 & 220.2 & 220.3 & 220.3 & & & \\
\hline 186,0 & 188.6 & 191,0 & 192.9 & 194.1 & 195.7 & 199.1 \\
\hline 204.1 & 209.0 & 212.4 & 215.9 & 218.1 & 219.9 & 221.2 \\
\hline 222.2 & 221.3 & 220.2 & 218.7 & 218.5 & 217.9 & 218.4 \\
\hline 218.7 & 219.2 & 219.7 & 219.8 & & & \\
\hline 183.9 & 186.7 & 189.8 & 191.7 & 192.4 & 193.0 & 196.4 \\
\hline 202.0 & $2(16.4$ & 211.5 & 2.15 .2 & 216.9 & 217.8 & 218.5 \\
\hline
\end{tabular}

A Ground Water Flow Model for the A/M Area of the SRS (U) 


$\begin{array}{lllllll}219.8 & 219.2 & 219.8 & 219.0 & 218.3 & 218.3 & 217.9 \\ 218.1 & 218.5 & 216.4 & 215.6 & & & \\ 181.2 & 184.1 & 188.3 & 190.7 & 190.9 & 191,9 & 195.7 \\ 200.6 & 203.8 & 208.6 & 212.5 & 214.0 & 214.5 & 214.1 \\ 214.6 & 216.1 & 217.2 & 217.5 & 217.5 & 217.5 & 217.5 \\ 217.7 & 215.9 & 215.1 & 214.3 & & & \\ 177.3 & 179.6 & 183.8 & 187.4 & 188.9 & 189.7 & 193.1 \\ 198.9 & 2.02 .2 & 205.6 & 208.7 & 210.0 & 211.1 & 210.3 \\ 208.9 & 214.5 & 214.7 & 216.1 & 216.5 & 216.7 & 216.8 \\ 215.7 & 214.6 & 213.9 & 213.4 & & & \\ 172.9 & 173.3 & 176.3 & 180.9 & 184.9 & 188.0 & 190.0 \\ 194.2 & 200.6 & 203.0 & 205.5 & 206.0 & 206.8 & 206.5 \\ 208.2 & 212.5 & 214.4 & 215.0 & 214.3 & 214.3 & 214.1 \\ 213.4 & 212.7 & 212.1 & 211.7 & & & \\ 169.4 & 169.1 & 171.6 & 175.8 & 180.1 & 184.3 & 187.2 \\ 190.7 & 199.1 & 200.8 & 202.8 & 202.8 & 202.4 & 203.5 \\ 205.9 & 209.0 & 211.5 & 211.9 & 212.2 & 212.0 & 211.3 \\ 210.8 & 210.3 & 209.9 & 209.9 & & & \\ 166.8 & 166.5 & 170.0 & 171.8 & 175.7 & 180.2 & 184.2 \\ 187.7 & 190.9 & 200.0 & 201.3 & 200.2 & 200.6 & 201.7 \\ 203.9 & 206.3 & 208.3 & 209.3 & 209.8 & 209.4 & 208.7 \\ 208.4 & 208.3 & 208.1 & 208.4 & & & \\ 166.1 & 165.8 & 163.3 & 168.7 & 173.5 & 176.9 & 181.8 \\ 186.2 & 187.6 & 191.2 & 198.8 & 199.6 & 199.7 & 200.4 \\ 201.6 & 203.7 & 205.9 & 207.4 & 207.6 & 206.7 & 206.2 \\ 206.5 & 206.8 & 207.2 & 201.9 & & & \\ 165.8 & 170.0 & 167.9 & 169.3 & 171.7 & 174.1 & 180.4 \\ 184.2 & 187.6 & 186.4 & 197.8 & 198.8 & 198.6 & 198.8 \\ 199.4 & 200.8 & 203.8 & 206.5 & 206.8 & 204.4 & 204.2 \\ 204.9 & 205.6 & 206.6 & 207.8 & & & \\ 162.4 & 165.7 & 168.4 & 170.6 & 172.3 & 173.9 & 175.3 \\ 182.8 & 185.4 & 187.6 & 189.3 & 198.0 & 197.9 & 198.1 \\ 198.0 & 198.8 & 202.1 & 205.2 & 206.0 & 204.2 & 203.0 \\ 203.2 & 204.3 & 206.1 & 207.9 & & & \\ 158.1 & 161.2 & 167.1 & 170.7 & 172.0 & 173.5 & 174.7 \\ 182.2 & 183.7 & 18.5 .7 & 186.8 & 197.7 & 188.2 & 189.4 \\ 190.9 & 193.0 & 195.4 & 197.5 & 198.4 & 200.0 & 200.1 \\ 200.9 & 202.8 & 205.4 & 208.1 & & & \\ 154.2 & 158.4 & 164.3 & 168.9 & 171.6 & 173.4 & 174.6 \\ 175.6 & 184.4 & 182.6 & 179.3 & 183.3 & 184.4 & 185.3 \\ 186.4 & 188.0 & 189.7 & 191.0 & 192.2 & 192.8 & 195.8 \\ 197.2 & 1999.6 & 203.3 & 207.3 & & & \\ 150.0 & 156.5 & 162.8 & 168.0 & 171.2 & 173.3 & 174.6 \\ 172.0 & 179.7 & 178.0 & 178.3 & 178.0 & 180.2 & 180.7 \\ 181.5 & 182.7 & 183.6 & 184.9 & 185.9 & 186.7 & 188.0 \\ 191.4 & 194.4 & 199.2 & 205.3 & & & \\ 152.2 & 156.8 & 162.8 & 168.1 & 171.4 & 171.5 & 171.6\end{array}$

A Ground Water Flow Model for the A/M Area of the SRS (U) 



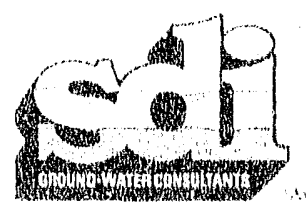

\begin{tabular}{|c|c|c|c|c|c|c|}
\hline 171.4 & 175.8 & 176.5 & 175.4 & 175.4 & 176.5 & 177.6 \\
\hline 177.6 & 178.0 & 178.2 & 178.3 & 179.1 & 179.9 & 180.9 \\
\hline 182.7 & 186.6 & 192.0 & 199.3 & & & \\
\hline 154.0 & 157.7 & 163.4 & 170.0 & 171.4 & 171.9 & 171.9 \\
\hline 171.7 & 174.2 & 173.9 & 173.9 & 174.1 & 174.3 & 173.7 \\
\hline 173.1 & 172.6 & 172.1 & 172.5 & 173.4 & 174.4 & 170.0 \\
\hline 171.9 & 175.7 & 178.6 & 185.6 & & & \\
\hline 155.7 & 159.4 & 164.9 & 169.7 & 171.2 & 171.3 & 171.0 \\
\hline 170.9 & 170.8 & 172.8 & 172.4 & 171.9 & 171.1 & 170.0 \\
\hline 168.2 & 167.0 & 166.0 & 166.3 & 162.6 & 164.2 & 164.9 \\
\hline 165.1 & 167.2 & 168.3 & 173.9 & & & \\
\hline 157.3 & 161.7 & 167.0 & 170.2 & 170.3 & 170.1 & 169.9 \\
\hline 169.5 & 168.8 & 167.9 & 167.1 & 169.0 & 167.9 & 166.0 \\
\hline 164.3 & 162.4 & 160.0 & 159.6 & 159.5 & 159.5 & 160.0 \\
\hline 160.5 & 161.0 & 162.7 & 166.1 & & & \\
\hline 157.4 & 161.9 & 165.3 & 167.6 & 167.0 & 167.8 & 167.6 \\
\hline 167.5 & 166.9 & 166.6 & 165.3 & 163.3 & 161.7 & 160.5 \\
\hline 159.3 & 158.7 & 157.4 & 157.1 & 157.0 & 156.9 & 155.7 \\
\hline 155.1 & 155.4 & 157.0 & 159.2 & & & \\
\hline 153.5 & 158.2 & 160.4 & 162.0 & 162.7 & 163.2 & 163.4 \\
\hline 163.5 & 164.2 & 164.8 & 163.3 & 161.0 & 159.3 & 157.6 \\
\hline 157.0 & 155.8 & 154.7 & 154.0 & 153.3 & 153.8 & 153.4 \\
\hline 151.3 & 150.7 & 152.1 & 153.3 & & & \\
\hline 148.4 & 152.1 & 154.9 & 156.7 & 158.0 & 158.0 & 158.5 \\
\hline 159.1 & 159.9 & 160.6 & 159.5 & 157.0 & 155.3 & 153.8 \\
\hline 153.5 & 151.2 & 151.9 & 149.8 & 147.5 & 150.0 & 149.8 \\
\hline 149.9 & 148.4 & 148.4 & 148.6 & & & \\
\hline 144.6 & 146.6 & 148.9 & 150.5 & 151.6 & 152.8 & 153.2 \\
\hline 153.5 & 154.2 & 153.3 & 152.7 & 150.9 & 150.0 & 149.3 \\
\hline 149.0 & 148.2 & $1,0.0$ & 144.5 & 140.3 & 138.8 & 143.0 \\
\hline 144.8 & 144.3 & 144.8 & 144.4 & & & \\
\hline 140.8 & 142.4 & 143.8 & 145.1 & 146.1 & 146.0 & 147.0 \\
\hline 147.3 & 147.7 & 146.5 & 146.0 & 144.3 & 143.1 & 142.8 \\
\hline 140.4 & 142.5 & 142.7 & 139.9 & 136.6 & 135.1 & 135.8 \\
\hline 136.8 & 138.6 & 141.1 & 141.2 & & & \\
\hline 137.2 & 137.6 & 139.0 & 139.1 & 139.7 & 140.2 & 140.8 \\
\hline 142.0 & 140.2 & 139.9 & 138.8 & 136.8 & 136.5 & 134.1 \\
\hline 135.6 & 137.1 & 137.3 & 135.8 & 133.5 & 131.4 & 130.0 \\
\hline 130.4 & 133.0 & 140.0 & 139.6 & & & \\
\hline 133.3 & 133.3 & 132.9 & 132.8 & 133.9 & 133.8 & 133.5 \\
\hline 134.1 & 134.6 & 134.4 & 133.2 & 130.0 & 128.7 & 129.7 \\
\hline 130.7 & 131.9 & 133.2 & 132.3 & 130.6 & 129.1 & 128.2 \\
\hline 129.1 & 131.7 & 135.6 & 137.3 & & & \\
\hline 129.7 & 129.2 & 129.0 & 128.8 & 127.5 & 128.5 & 128.7 \\
\hline 129.0 & 129.2 & 129.5 & 130.0 & 127.3 & 125.5 & 125.8 \\
\hline 126.0 & 124.6 & 130.0 & 130.5 & 128.8 & 127.6 & 127.5 \\
\hline 120.1 & $13 ! n$ & 133.4 & 135.1 & & & \\
\hline
\end{tabular}




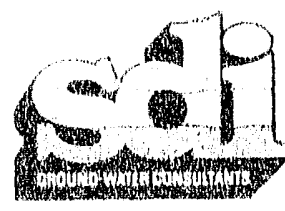

$\begin{array}{llrrrrr}126.1 & 125.7 & 125.6 & 124.9 & 124.3 & 121.5 & 121.7 \\ 122.2 & 124.4 & 123.8 & 124.5 & 123.3 & 121.2 & 123.9 \\ 126.4 & 123.5 & 126.1 & 128.3 & 127.6 & 128.1 & 128.4 \\ 128.3 & 130.3 & 131.7 & 133.7 & & & \\ 122.9 & 122.5 & 122.5 & 122.4 & 120.6 & 120.4 & 120.0 \\ 120.5 & 120.6 & 120.0 & 121.0 & 120.5 & 121.1 & 125.9 \\ 129.7 & 128.2 & 126.8 & 127.6 & 128.8 & 129.1 & 128.3 \\ 127.6 & 128.8 & 131.1 & 152.7 & & & \\ 120.0 & 120.0 & 120.7 & 120.7 & 120.0 & 119.8 & 119.7 \\ 119.8 & 119.8 & 119.6 & 119.8 & 119.8 & 120.0 & 130.0 \\ 130.0 & 129.4 & 128.7 & 128.5 & 150.0 & 151.5 & 152.2 \\ 154.3 & 155.2 & 156.2 & 158.4 & & & \\ 6 & 0.100 \mathrm{E}+01(7 \mathrm{G} 11.4) & & 12 . & & \\ 174.7 & 176.2 & 177.7 & 179.5 & 181.6 & 183.8 & 186.1 \\ 188.4 & 190.7 & 192.9 & 195.2 & 197.4 & 199.6 & 201.7 \\ 203.9 & 206.4 & 208.9 & 210.9 & 212.2 & 213.3 & 214.0 \\ 214.4 & 214.7 & 214.8 & 214.6 & & & \\ 173.5 & 175.0 & 176.7 & 178.6 & 180.7 & 182.8 & 185.1 \\ 187.5 & 189.8 & 192.1 & 194.3 & 196.4 & 198.4 & 200.4 \\ 202.8 & 205.5 & 208.3 & 210.6 & 212.3 & 213.4 & 214.2 \\ 214.6 & 214.8 & 214.9 & 214.8 & & & \\ 172.2 & 173.8 & 175.6 & 177.6 & 179.6 & 181.8 & 184.1 \\ 186.5 & 188.9 & 191.2 & 193.2 & 195.2 & 197.1 & 199.1 \\ 201.4 & 204.3 & 207.3 & 210.2 & 212.2 & 213.4 & 214.2 \\ 214.7 & 214.8 & 214.8 & 214.7 & & & \\ 170.9 & 172.7 & 174.6 & 176.6 & 178.6 & 180.9 & 183.2 \\ 185.5 & 187.9 & 190.2 & 192.2 & 194.0 & 195.9 & 197.7 \\ 199.9 & 202.6 & 205.9 & 209.3 & 211.8 & 213.3 & 214.1 \\ 214.5 & 214.6 & 214.6 & 214.4 & & & \\ 170.1 & 171.8 & 173.7 & 175.7 & 177.8 & 180.1 & 182.2 \\ 184.4 & 186.7 & 188.9 & 191.0 & 192.8 & 194.5 & 196.3 \\ 198.1 & 200.4 & 203.7 & 207.7 & 211.1 & 212.8 & 213.7 \\ 214.1 & 214.2 & 214.2 & 214.0 & & & \\ 169.5 & 171.1 & 172.8 & 174.8 & 176.9 & 179.0 & 181.1 \\ 183.1 & 185.3 & 187.5 & 189.6 & 191.5 & 193.2 & 194.8 \\ 196.4 & 198.3 & 200.9 & 205.0 & 209.5 & 211.9 & 212.9 \\ 213.3 & 213.5 & 213.4 & 213.3 & & & \\ 169.0 & 170.3 & 172.0 & 173.9 & 175.8 & 177.8 & 179.7 \\ 181.7 & 183.7 & 185.9 & 188.1 & 190.2 & 191.8 & 193.3 \\ 194.9 & 196.6 & 198.4 & 201.4 & 206.4 & 209.9 & 211.5 \\ 212.1 & 212.4 & 212.5 & 212.4 & & & \\ 168.3 & 169.6 & 171.2 & 173.0 & 174.8 & 176.6 & 178.4 \\ 180.1 & 182.9 & 184.2 & 186.4 & 188.6 & 190.3 & 191.9 \\ 193.6 & 195.3 & 197.1 & 199.4 & 203.5 & 207.3 & 209.4 \\ 210.4 & 211.0 & 211.2 & 211.2 & & & \\ 167.8 & 169.0 & 170.5 & 172.2 & 173.9 & 175.5 & 177.1 \\ 178.7 & 180.5 & 182.5 & 184.7 & 186.8 & 188.7 & 190.6\end{array}$

A Ground Water Flow Model for the A/M Area of the SRS (U) 


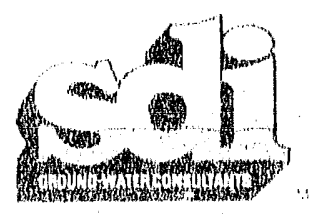

$\begin{array}{lllllll}192.5 & 194.4 & 196.4 & 198.8 & 201.9 & 204.8 & 207.0 \\ 208.4 & 209.2 & 209.6 & 209.7 & & & \\ 167.2 & 168.4 & 169.9 & 171.4 & 173.0 & 174.6 & 176.1 \\ 177.6 & 179.1 & 180.9 & 183.0 & 185.2 & 187.4 & 189.6 \\ 191.6 & 193.5 & 195.6 & 198.0 & 200.6 & 202.8 & 204.7 \\ 206.1 & 207.0 & 207.5 & 207.8 & & & \\ 166.5 & 167.8 & 169.3 & 170.8 & 172.3 & 173.7 & 175.2 \\ 176.6 & 178.0 & 179.6 & 181.5 & 183.8 & 186.2 & 188.5 \\ 190.5 & 192.5 & 194.5 & 196.7 & 198.9 & 200.9 & 202.5 \\ 203.7 & 204.7 & 205.3 & 205.7 & & & \\ 165.9 & 167.2 & 168.6 & 170.1 & 171.6 & 173.0 & 174.4 \\ 175.8 & 177.2 & 178.7 & 180.5 & 182.6 & 184.9 & 187.2 \\ 189.4 & 191.3 & 193.2 & 195.1 & 196.9 & 198.7 & 200.1 \\ 201.4 & 202.4 & 203.1 & 203.6 & & & \\ 165.1 & 166.4 & 167.9 & 169.5 & 171.0 & 172.4 & 173.8 \\ 175.2 & 176.6 & 178.0 & 179.6 & 181.6 & 183.8 & 185.9 \\ 188.0 & 189.9 & 191.6 & 193.3 & 194.9 & 196.3 & 197.7 \\ 198.9 & 200.0 & 200.9 & 201.4 & & & \\ 164.3 & 165.6 & 167.2 & 168.8 & 170.4 & 171.8 & 173.3 \\ 174.6 & 176.0 & 177.4 & 178.9 & 180.7 & 182.8 & 184.7 \\ 186.6 & 188.4 & 190.0 & 191.4 & 192.7 & 194.0 & 195.2 \\ 196.4 & 197.4 & 198.4 & 199.0 & & & \\ 163.4 & 164.8 & 166.4 & 168.1 & 169.8 & 171.3 & 172.8 \\ 174.2 & 175.5 & 176.9 & 178.5 & 180.1 & 181.9 & 183.6 \\ 185.4 & 187.0 & 188.4 & 189.6 & 190.6 & 191.7 & 192.8 \\ 193.9 & 194.9 & 195.8 & 196.5 & & & \\ 162.4 & 163.9 & 165.6 & 167.4 & 169.3 & 170.9 & 172.3 \\ 1173.7 & 175.1 & 176.5 & 177.9 & 179.5 & 181.0 & 182.6 \\ 184.2 & 185.6 & 186.9 & 187.9 & 188.8 & 189.7 & 190.6 \\ 191.5 & 192.4 & 193.3 & 194.1 & & & \\ 161.3 & 162.9 & 164.8 & 166.7 & 168.7 & 170.4 & 171.9 \\ 173.3 & 174.6 & 176.0 & 177.4 & 178.8 & 180.2 & 181.7 \\ 183.1 & 184.4 & 185.5 & 186.5 & 187.3 & 188.1 & 188.8 \\ 189.4 & 190.2 & 191.0 & 191.8 & & & \\ 160.4 & 162.1 & 164.0 & 166.0 & 167.9 & 169.8 & 171.4 \\ 172.8 & 174.1 & 175.5 & 176.8 & 178.1 & 179.5 & 180.8 \\ 182.2 & 183.3 & 184.4 & 185.3 & 186.0 & 186.7 & 187.4 \\ 188.0 & 188.6 & 189.2 & 189.9 & & & \\ 159.9 & 161.4 & 163.3 & 165.3 & 167.3 & 169.2 & 170.8 \\ 172.3 & 173.6 & 174.9 & 176.2 & 177.5 & 178.8 & 180.1 \\ 181.3 & 182.4 & 183.3 & 184.2 & 184.9 & 185.6 & 186.2 \\ 186.8 & 187.4 & 188.0 & 188.6 & & & \\ 159.5 & 160.9 & 162.7 & 164.6 & 166.6 & 168.6 & 170.3 \\ 171.8 & 173.1 & 174.3 & 175.6 & 176.8 & 178.1 & 179.4 \\ 180.6 & 181.6 & 182.4 & 183.2 & 183.9 & 184.5 & 185.1 \\ 185.7 & 186.3 & 187.0 & 187.7 & & & \\ 159.2 & 160.4 & 162.1 & 164.0 & 166.0 & 167.9 & 169.8 \\ & & & & & & \end{array}$

A Ground Water Flow Model for the A/M Area of the SRS (U) 


$\begin{array}{lllllll}171.2 & 172.5 & 173.7 & 174.9 & 176.1 & 177.4 & 178.6 \\ 179.8 & 180.7 & 181.5 & 182.3 & 183.0 & 183.6 & 184.2 \\ 184.8 & 185.4 & 186.1 & 186.7 & & & \\ 159.0 & 160.1 & 161.6 & 163.4 & 165.3 & 167.2 & 169.0 \\ 170.5 & 171.7 & 172.9 & 174.1 & 175.3 & 176.5 & 177.7 \\ 178.8 & 179.8 & 180.6 & 181.4 & 182.1 & 182.7 & 183.4 \\ 184.0 & 184.6 & 185.2 & 185.9 & & & \\ 158.9 & 159.8 & 161.2 & 162.9 & 164.6 & 166.4 & 168.1 \\ 169.6 & 170.9 & 172.1 & 173.3 & 174.4 & 175.6 & 176.7 \\ 177.8 & 178.8 & 179.7 & 180.5 & 181.2 & 181.9 & 182.5 \\ 183.1 & 183.8 & 184.4 & 185.1 & & & \\ 158.7 & 159.6 & 160.8 & 162.3 & 164.0 & 165.6 & 167.2 \\ 168.7 & 170.0 & 171.2 & 172.4 & 173.5 & 174.7 & 175.8 \\ 176.8 & 177.8 & 178.7 & 179.6 & 180.4 & 181.1 & 181.7 \\ 182.3 & 183.0 & 183.6 & 184.3 & & & \\ 158.6 & 159.4 & 160.5 & 161.8 & 163.4 & 164.9 & 166.4 \\ 167.8 & 169.1 & 170.3 & 171.4 & 172.6 & 173.7 & 174.8 \\ 175.9 & 176.9 & 177.8 & 178.7 & 179.5 & 180.3 & 180.9 \\ 181.6 & 182.2 & 182.8 & 183.5 & & & \\ 158.5 & 159.2 & 160.2 & 161.4 & 162.8 & 164.3 & 165.7 \\ 167.0 & 168.3 & 169.4 & 170.6 & 171.7 & 172.8 & 173.9 \\ 174.9 & 175.9 & 176.9 & 177.8 & 178.6 & 179.4 & 180.1 \\ 180.8 & 181.4 & 182.0 & 182.7 & & & \\ 158.4 & 159.1 & 160.0 & 161.1 & 162.4 & 163.7 & 165.1 \\ 166.3 & 167.5 & 168.6 & 169.7 & 170.8 & 171.9 & 173.0 \\ 174.1 & 175.0 & 176.0 & 176.9 & 177.7 & 178.5 & 179.3 \\ 180.0 & 180.6 & 181.3 & 181.9 & & & \\ 158.4 & 159.1 & 159.9 & 160.9 & 162.1 & 163.3 & 164.5 \\ 165.7 & 166.9 & 168.0 & 169.0 & 170.1 & 171.1 & 172.2 \\ 173.2 & 174.2 & 175.2 & 176.1 & 176.9 & 177.7 & 178.5 \\ 179.2 & 179.8 & 180.5 & 181.1 & & & \\ 158.4 & 159.0 & 159.8 & 160.7 & 161.8 & 162.9 & 164.0 \\ 165.2 & 166.3 & 167.3 & 168.4 & 169.4 & 170.4 & 171.4 \\ 172.4 & 173.4 & 174.4 & 175.3 & 176.1 & 176.9 & 177.7 \\ 178.4 & 179.1 & 179.7 & 180.3 & & & \\ 158.4 & 159.0 & 159.7 & 160.6 & 161.6 & 162.6 & 163.6 \\ 164.7 & 165.7 & 166.8 & 167.8 & 168.8 & 169.8 & 170.7 \\ 171.7 & 172.7 & 173.6 & 174.6 & 175.4 & 176.2 & 177.0 \\ 177.7 & 178.4 & 179.0 & 179.6 & & & \\ 158.4 & 159.0 & 159.7 & 160.5 & 161.4 & 162.3 & 163.3 \\ 164.3 & 165.3 & 166.4 & 167.3 & 168.3 & 169.2 & 170.1 \\ 171.1 & 172.0 & 173.0 & 173.9 & 174.7 & 175.5 & 176.3 \\ 177.0 & 177.7 & 178.3 & 179.0 & & & \\ 158.5 & 150.0 & 159.6 & 160.4 & 161.3 & 162.1 & 163.1 \\ 164.0 & 165.0 & 165.9 & 166.9 & 167.8 & 168.7 & 169.6 \\ 170.4 & 171.4 & 172.4 & 173.3 & 174.1 & 174.9 & 175.7 \\ 176.4 & 177.1 & 177.7 & 178.4 & & & \end{array}$

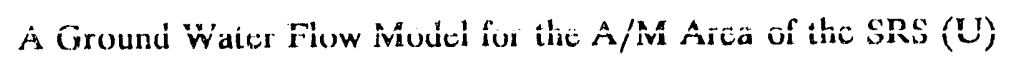




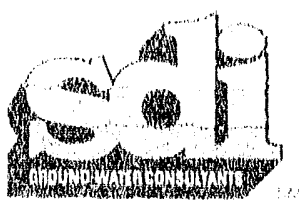

\begin{tabular}{rrrrrrr}
158.5 & 159.0 & 159.6 & 160.3 & 161.1 & 162.0 & 162.9 \\
163.8 & 164.7 & 165.6 & 166.6 & 167.5 & 168.3 & 169.1 \\
170.0 & 170.9 & 171.9 & 172.8 & 173.6 & 174.4 & 175.1 \\
175.8 & 176.5 & 177.1 & 177.8 & & & \\
158.6 & 159.0 & 159.6 & 160.2 & 161.0 & 161.8 & 162.7 \\
163.6 & 164.5 & 165.4 & 166.3 & 167.2 & 168.0 & 168.9 \\
169.7 & 170.6 & 171.5 & 172.3 & 173.1 & 173.9 & 174.6 \\
175.2 & 175.9 & 176.6 & 177.2 & & & \\
158.8 & 159.1 & 159.6 & 160.2 & 160.9 & 161.8 & 162.6 \\
163.5 & 164.3 & 165.2 & 166.0 & 166.9 & 167.8 & 168.7 \\
169.6 & 170.4 & 171.2 & 172.0 & 172.7 & 173.4 & 174.1 \\
174.7 & 175.4 & 176.0 & 176.7 & & & \\
1.0000 & \multicolumn{1}{c}{11.0000} & & & &
\end{tabular}




\section{APPEND!X C2}

Block Centered Flow Package Nodal Spacing, Transmissivity, and Leakance 


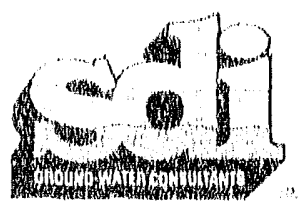

WSSC.BCF

$\begin{array}{rll} & 1 & -1 \\ 000 & 0 & \\ 0 & 0.100 \mathrm{E}+01 \\ & 0 & 0.100 \mathrm{E}+04 \\ 0 & 0.100 \mathrm{E}+04 \\ 0 & 0.175 \mathrm{E}+03\end{array}$

$110.100 \mathrm{E}+01(7 \mathrm{G} 11.4)$

12

$0.2000 \mathrm{E}-02,0.2000 \mathrm{E}-02 \quad 0.2000 \mathrm{E}-02 \quad 0.2000 \mathrm{E}-02 \quad 0.2000 \mathrm{E}-02 \quad 0.2000 \mathrm{E}-02 \quad 0.2000 \mathrm{E}-02$ $0.2000 \mathrm{E}-02 \quad 0.2000 \mathrm{E}-02 \quad 0.2000 \mathrm{E}-02 \quad 0.2000 \mathrm{E}-02 \quad 0.2000 \mathrm{E}-02 \quad 0.2000 \mathrm{E}-02 \quad 0.2000 \mathrm{E}-02$ $0.2000 \mathrm{E}-02 \quad 0.2000 \mathrm{E}-02$ (1.2000E-02 0.2000E-02 0.2000E-02 0.2000E-02 0.2200E-03 $0.2200 \mathrm{E}-03 \quad 0.2200 \mathrm{E}-03$ - $16200 \mathrm{E}-03 \quad 0.2200 \mathrm{E}-03$

$0.2000 \mathrm{E}-02 \quad 0.2000 \mathrm{E}-02$ (6) $0.2000 \mathrm{E}-02 \quad 0.2000 \mathrm{E}-02 \quad(0.2000 \mathrm{E}-020.2000 \mathrm{E}-02 \quad 0.2000 \mathrm{E}-02 \quad 0.2000 \mathrm{E}-02 \quad 0.2000 \mathrm{E}-02$ $0.2000 \mathrm{E}-02 \quad 0.2000 \mathrm{E}-02 \quad 0.000 \mathrm{E}-020.2000 \mathrm{E}-02 \quad 0.2000 \mathrm{E}-02 \quad 0.2000 \mathrm{E}-02 \quad 0.2200 \mathrm{E}-03$ $0.2200 \mathrm{E}-03 \quad 0.2200 \mathrm{E}-03 \quad 0.2200 \mathrm{E}-03 \quad 0.2200 \mathrm{E}-03$

$0.2000 \mathrm{E}-02 \quad 0.2000 \mathrm{E}-02 \quad 0.2000 \mathrm{E}-02 \quad 0.2000 \mathrm{E}-02 \quad 0.2000 \mathrm{E}-02,0.2000 \mathrm{E}-02 \quad 0.2000 \mathrm{E}-02$ $0.2000 \mathrm{E}-02 \quad 0.2000 \mathrm{E}-02 \quad 0 . .000 \mathrm{E}-02 \quad 0.2000 \mathrm{E}-02 \quad 0.2000 \mathrm{E}-02 \quad 0.2000 \mathrm{E}-02 \quad 0.2000 \mathrm{E}-02$ $0.2000 \mathrm{E}-02 \quad 0.2000 \mathrm{E}-02 \quad 0.4000 \mathrm{E}-02 \quad 0.2000 \mathrm{E}-02 \quad 0.2000 \mathrm{E}-02 \quad 0.2000 \mathrm{E}-02 \quad 0.2200 \mathrm{E}-03$ $0.2200 \mathrm{E}-03 \quad 0.2200 \mathrm{E}-03 \quad 0.2200 \mathrm{E}-03 \quad 0.2200 \mathrm{E}-03$

$0.2000 \mathrm{E}-02 \quad 0.2000 \mathrm{E}-02 \quad 0.2000 \mathrm{E}-02 \quad 0.2000 \mathrm{E}-02 \quad 0.2000 \mathrm{E}-02 \quad 0.2000 \mathrm{E}-02 \quad 0.2000 \mathrm{E}-02$ $0.2000 \mathrm{E}-02 \quad 0.2000 \mathrm{E}-02 \quad 0.2000 \mathrm{E}-02 \quad 0.2000 \mathrm{E}-02 \quad 0.2000 \mathrm{E}-020.2000 \mathrm{E}-02 \quad 0.2000 \mathrm{E}-02$ $0.2000 \mathrm{E}-02,0.2000 \mathrm{E}-020.2000 \mathrm{E}-020.2000 \mathrm{E}-02 \quad 0.1800 \mathrm{E}-03 \quad 0.2200 \mathrm{E}-03 \quad 0.2200 \mathrm{E}-03$ $0.2200 \mathrm{E}-03 \quad 0.2200 \mathrm{E}-03$ (2200E:-03 $0.2200 \mathrm{E}-03$

$0.2000 \mathrm{E}-02,0.2000 \mathrm{E}-020.2000 \mathrm{E}-020.2000 \mathrm{E}-02 \quad 0.2000 \mathrm{E}-020.2000 \mathrm{E}-02 \quad 0.2000 \mathrm{E}-02$ $0.2000 \mathrm{E}-02 \quad 0.2000 \mathrm{E}-02 \quad 0.2000 \mathrm{E}-02 \quad 0.20000 \mathrm{E}-02 \quad 0.2000 \mathrm{E}-02 \quad 0.2000 \mathrm{E}-02 \quad 0.2000 \mathrm{E}-02$ 0.2000 E- $02 \quad 0.1800 \mathrm{E}-03$ (1, 1800E-03 $0.1800 \mathrm{E}-03$ 0.1800E-03 0.1800E-03 $0.2200 \mathrm{E}-03$ $0.2200 \mathrm{E}-03 \quad 0.2200 \mathrm{E} \cdot() 3 \quad 0.2200 \mathrm{E}-030.2200 \mathrm{E}-0.3$

$0.2000 \mathrm{E}-02 \quad 0.2000 \mathrm{E}-02,0.2000 \mathrm{E}-02 \quad 0.2000 \mathrm{E}-02 \quad 0.2000 \mathrm{E}-02 \quad 0.2000 \mathrm{E}-02 \quad 0.2000 \mathrm{E}-02$ $0.2000 \mathrm{E}-02 \quad 0.2000 \mathrm{E}-02,0.2000 \mathrm{E}-02 \quad 0.2000 \mathrm{E}-02 \quad 0.2000 \mathrm{E}-02 \quad 0.2000 \mathrm{E}-02 \quad 0.2000 \mathrm{E}-02$ 0.1800 E- 03 0.1800E-03 0.1800 E-03 $0.1800 \mathrm{E}-03 \quad 0.1800 \mathrm{E}-0.30 .1800 \mathrm{E}-03 \quad 0.2200 \mathrm{E}-03$ $0.2200 \mathrm{E}-03 \quad 0.2200 \mathrm{E}-030.2200 \mathrm{E}-030.2200 \mathrm{E}-03$

$0.2000 \mathrm{E}-02 \quad 0.2000 \mathrm{E}-(02,0.2000) \mathrm{E}-02 \quad 0.2000 \mathrm{E}-02 \quad 0.2000 \mathrm{E}-02 \quad 0.2000 \mathrm{E}-02 \quad 0.2000 \mathrm{E}-02$ $0.2000 \mathrm{E}-02 \quad 0.200(0 \mathrm{E}-02) 0.2000 \mathrm{E}-020.2000 \mathrm{E}-02 \quad 0.2000 \mathrm{E}-02 \quad 0.2000 \mathrm{E}-02 \quad 0.2000 \mathrm{E}-02$ $0.1800 \mathrm{E}-03 \quad 0.1800 \mathrm{E}-(0) \quad 0.1800 \mathrm{E}-0.30 .1800 \mathrm{E}-03 \quad 0.1800 \mathrm{E}-0.30 .1800 \mathrm{E}-03 \quad 0.2200 \mathrm{E}-(03$ $0.2200) \mathrm{E}-0.30 .22(0) \mathrm{E}-0.1) 0.2200 \mathrm{E}-03$ 0.3420E-03

$0.200(0 \mathrm{E}-020.2000 \mathrm{E}-0.20 .2000 \mathrm{E}-02 \quad 0.2000 \mathrm{E}-02 \quad 0.2000 \mathrm{E}-020.2000 \mathrm{E}-02 \quad 0.2000 \mathrm{E}-02$

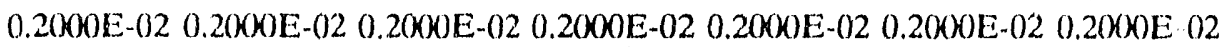
0.1800 E- 03 0.1800)E-03 0.1800 E- 03 0.1800E-03 $0.1800 \mathrm{E}-03 \quad 0.1800 \mathrm{E}-03 \quad 0.2200 \mathrm{E}-03$ $0.2200 \mathrm{E}-(03)(0.2200 \mathrm{E}-030.2200 \mathrm{E}-0.30 .3420 \mathrm{E}-03$

$0.200) \mathrm{E}-(020.20(0) \mathrm{E}-(02 \quad 0.2000 \mathrm{E}-02 \quad 0.2000 \mathrm{E}-02 \quad 0.2000 \mathrm{E}-020.2000 \mathrm{E}-020.2000 \mathrm{E}-02$ $0.2000 \mathrm{E}-(020.2000 \mathrm{E}-020.2000 \mathrm{E}-020.2000 \mathrm{E}-02 \quad 0.3420 \mathrm{E}-03 \quad 0.3420 \mathrm{E}-03 \quad 0.3420 \mathrm{E}-03$ $0.1800 \mathrm{E}-0.30 .1800 \mathrm{E}-03$ 0.1800E-03 $0.1800 \mathrm{E}-03$ 0.1800E-03 $0.1800 \mathrm{E}-03$ 0.2200E-03 $0.2200 \mathrm{E}-0.30 .22(\mathrm{~K}) \mathrm{E}-030.2200 \mathrm{E}-0.30 .3420 \mathrm{E}-03$

$0.2000 \mathrm{E}-02 \quad 0.2000 \mathrm{E}-02 \quad 0.2000 \mathrm{E}-02 \quad 0.2000 \mathrm{E}-02 \quad 0.2000 \mathrm{E}-020.2000 \mathrm{E}-02 \quad 0.2000 \mathrm{E}-02$

A Ground Water Flow Model for the A/M Area of the SRS (U)

$\mathrm{C} 2 n 1$ 


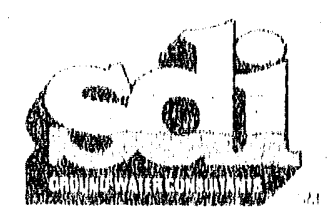

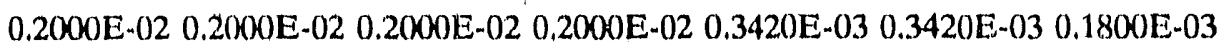
$0.1800 \mathrm{E}-03 \quad 0.1800 \mathrm{E}-03 \quad 0.1800 \mathrm{E}-03 \quad 0.1800 \mathrm{E}-03 \quad 0.1800 \mathrm{E}-03 \quad 0.1800 \mathrm{E}-03 \quad 0.3420 \mathrm{E}-03$ $0.3420 \mathrm{E}-03 \quad 0.3420 \mathrm{E}-03 \quad 0.3420 \mathrm{E}-03 \quad 0.3420 \mathrm{E}-03$

$0.2000 \mathrm{E}-02 \quad 0.2000 \mathrm{E}-02 \quad 0.2000 \mathrm{E}-02 \quad 0.2000 \mathrm{E}-02 \quad 0.2000 \mathrm{E}-02 \quad 0.2000 \mathrm{E}-02 \quad 0.2000 \mathrm{E}-02$ $0.2000 \mathrm{E}-02 \quad 0.2000 \mathrm{E}-02 \quad 0.3420 \mathrm{E}-03 \quad 0.3420 \mathrm{E}-03 \quad 0.3420 \mathrm{E}-03 \quad 0.1800 \mathrm{E}-0.30 .1800 \mathrm{E}-03$ $0.1800 \mathrm{E}-03 \quad 0.1800 \mathrm{E}-03$ 0.1800E-03 $0.1800 \mathrm{E}-03$ 0.1800E-03 0.3420E-03 0.342.0E-03 $0.3420 \mathrm{E}-03 \quad 0.3420 \mathrm{E}-0.3 \quad 0.3420 \mathrm{E}-03 \quad 0.3420 \mathrm{E}-03$

0.2000E-02 0.2000E-02 0.2000E-02 0.2000E-02 0,2000E-02 0.2000E-02 0.2000E-02 $0.2000 \mathrm{E}-02, \quad 0.2000 \mathrm{E}-02 \quad 0.3420 \mathrm{E}-03 \quad 0.3420 \mathrm{E}-03 \quad 0.3420 \mathrm{E}-03 \quad 0.1800 \mathrm{E}-03 \quad 0.1800 \mathrm{E}-03$ $0.1800 \mathrm{E}-03 \quad 0.1800 \mathrm{E}-03 \quad 0.1800 \mathrm{E}-03 \quad 0.1800 \mathrm{E}-03 \quad 0.1800 \mathrm{E}-03 \quad 0.3420 \mathrm{E}-03 \quad 0.3420 \mathrm{E}-03$ $0.3420 \mathrm{E}-03 \quad 0.3420 \mathrm{E}-03 \quad 0.3420 \mathrm{E}-03 \quad 0.3420 \mathrm{E}-03$

$0.2000 \mathrm{E}-02 \quad 0.2000 \mathrm{E}-02 \quad 0.2000 \mathrm{E}-02 \quad 0.2000 \mathrm{E}-02 \quad 0.2000 \mathrm{E}-02 \quad 0.2000 \mathrm{E}-02 \quad 0.2000 \mathrm{E}-02$ $0.2000 \mathrm{E}-02 \quad 0.2000 \mathrm{E}-02 \quad 0.3420 \mathrm{E}-03 \quad 0.3420 \mathrm{E}-03 \quad 0.3420 \mathrm{E}-03 \quad 0.1800 \mathrm{E}-03 \quad 0.1800 \mathrm{E}-03$ $0.1800 \mathrm{E}-03 \quad 0.1800 \mathrm{E}-03 \quad 0.3420 \mathrm{E}-03 \quad 0.3420 \mathrm{E}-03$ 0.3420E-03 $0.3420 \mathrm{E}-03 \quad 0.3420 \mathrm{E}-03$ $0.3420 \mathrm{E}-03 \quad 0.3420 \mathrm{E}-03 \quad 0.3420 \mathrm{E}-03 \quad 0.3420 \mathrm{E}-03$

$0.2000 \mathrm{E}-(02 \quad 0.200) \mathrm{E}-02 \quad 0.2000 \mathrm{E}-02 \quad 0.2000 \mathrm{E}-02 \quad 0.2000 \mathrm{E}-02 \quad 0.2000 \mathrm{E}-02 \quad 0.2000 \mathrm{E}-02$ $0.2000 \mathrm{E}-02 \quad 0.2000 \mathrm{E}-02 \quad 0.3420 \mathrm{E}-03 \quad 0.3420 \mathrm{E}-03 \quad 0.3420 \mathrm{E}-03 \quad 0.3420 \mathrm{E}-0.30 .3420 \mathrm{E}-03$ $0.3420 \mathrm{E}-03 \quad 0.3420 \mathrm{E}-(03 \quad 0.3420 \mathrm{E}-03 \quad 0.3420 \mathrm{E}-03 \quad 0.3420 \mathrm{E}-03 \quad 0.3420 \mathrm{E}-03 \quad 0.3420 \mathrm{E}-03$ $0.3420 \mathrm{E}-03 \quad 0.3420 \mathrm{E}-03 \quad 0.3420 \mathrm{E}-03 \quad 0.3420 \mathrm{E}-03$

$0.2000 \mathrm{E}-02 \quad 0.2000 \mathrm{E}-02 \quad 0.2000 \mathrm{E}-02 \quad 0.2000 \mathrm{E}-02 \quad 0.2000 \mathrm{E}-02$ ().2000E-02 $0.2000 \mathrm{E}-02$ $0.2000 \mathrm{E}-02 \quad 0.2000 \mathrm{E}-02 \quad 0.3420 \mathrm{E}-03 \quad 0.3420 \mathrm{E}-03 \quad 0.3420 \mathrm{E}-03 \quad 0.3420 \mathrm{E}-03 \quad 0.3420 \mathrm{E}-03$ $0.3420 \mathrm{E}-03 \quad 0.3420 \mathrm{E}-03 \quad 0.3420 \mathrm{E}-03 \quad 0.3420 \mathrm{E}-03 \quad 0.3420 \mathrm{E}-03 \quad 0.3420 \mathrm{E}-03 \quad 0.3420 \mathrm{E}-03$ $0.3420 \mathrm{E}-030.3420 \mathrm{E}-03 \quad 0.3420 \mathrm{E}-0330.3420 \mathrm{E}-03$

$0.0000 \mathrm{E}+000.0000 \mathrm{E}+000.0000 \mathrm{E}+000.0000 \mathrm{E}+000.0000 \mathrm{E}+000.0000 \mathrm{E}+000.0000 \mathrm{E}+00$ $0.0000 \mathrm{E}+000.0000 \mathrm{E}+000.0000 \mathrm{E}+000.0000 \mathrm{E}+000.0000 \mathrm{E}+000.0000 \mathrm{E}+000.0000 \mathrm{E}+00$ $0.0000 \mathrm{E}+(0) 0.0000 \mathrm{E}+000.0000 \mathrm{E}+000.0000 \mathrm{E}+000.0000 \mathrm{E}+000.0000 \mathrm{E}+000.0000 \mathrm{E}+00$ $0.0000 \mathrm{E}+000.0000 \mathrm{E}+000.0000 \mathrm{E}+000.0000 \mathrm{E}+00$

$0.0000 \mathrm{E}+000.00000 \mathrm{E}+000.0000 \mathrm{E}+000.0000 \mathrm{E}+000.0000 \mathrm{E}+000.0000 \mathrm{E}+000.0000 \mathrm{E}+00$ $0.0000 \mathrm{E}+000.0000 \mathrm{E}+000.0000 \mathrm{E}+000.0000 \mathrm{E}+000.00000 \mathrm{E}+000.0000 \mathrm{E}+000.0000 \mathrm{E}+00$ $0.0000 \mathrm{E}+000.0000 \mathrm{E}+000.0000 \mathrm{E}+000.0000 \mathrm{E}+000.0000 \mathrm{E}+000.0000 \mathrm{E}+000.0000 \mathrm{E}+00$ $0.0000 \mathrm{E}+000.0000 \mathrm{E}+000.0000 \mathrm{E}+000.0000 \mathrm{E}+00$

$0.0000 \mathrm{E}+000.0000 \mathrm{E}+000.0000 \mathrm{E}+000.0000 \mathrm{E}+000.0000 \mathrm{E}+000.0000 \mathrm{E}+000.0000 \mathrm{E}+00$ $0.0000 \mathrm{E}+000.0000 \mathrm{E}+000.0000 \mathrm{E}+000.0000 \mathrm{E}+000.0000 \mathrm{E}+000.0000 \mathrm{E}+000.0000 \mathrm{E}+00$ $0.0000 \mathrm{E}+000.0000 \mathrm{E}+000.0000) \mathrm{E}+000.0000 \mathrm{E}+000.0000 \mathrm{E}+000.0000 \mathrm{E}+000.0000 \mathrm{E}+00$ $0.0000 \mathrm{E}+000.0000 \mathrm{E}+000.0 .0000 \mathrm{E}+000.0000 \mathrm{E}+00$

$0.0000 \mathrm{E}+000.0000 \mathrm{E}+000.0000 \mathrm{E}+1000.0000 \mathrm{E}+000.0000 \mathrm{E}+000.0000 \mathrm{E}+000.0000 \mathrm{E}+00$ $0.0000 \mathrm{E}+000.0000 \mathrm{E}+000.0000 \mathrm{E}+000.0000 \mathrm{E}+000.0000 \mathrm{E}+000.0000 \mathrm{E}+000.0000 \mathrm{E}+00$ $0.0000 \mathrm{E}+000.0000 \mathrm{E}+000.0000 \mathrm{E}+000.0000 \mathrm{E}+000.0000 \mathrm{E}+000.000(0) \mathrm{E}+000.0000 \mathrm{E}+00$ $0.0 .0(0) \mathrm{E}+000.0 .0(100 \mathrm{E}+0000.0000 \mathrm{E}+0000.0000 \mathrm{E}+00$

$0.0000 \mathrm{E}+0000.00000 \mathrm{E}+000.00000 \mathrm{E}+0000.0000 \mathrm{E}+0000.00000 \mathrm{E}+0000.00000 \mathrm{E}+000.0000 \mathrm{E}+00$

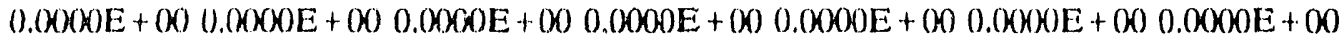
$0.0000 \mathrm{E}+000.0000 \mathrm{E}+000.0000 \mathrm{E}+000.0000 \mathrm{E}+000.00000 \mathrm{E}+000.00000 \mathrm{E}+000.00000 \mathrm{E}+00$ $0.0000 \mathrm{E}+(\mathrm{X}) 0.0000 \mathrm{E}+000.00000 \mathrm{E}+000.0000 \mathrm{E}+0.0$

$0.0000 \mathrm{E}+000.0000 \mathrm{E}+000.0000 \mathrm{E}+000.0000 \mathrm{E}+000.0000 \mathrm{E}+000.0000 \mathrm{E}+000.0000 \mathrm{E}+00$ $0.00 K 0) \mathrm{E}+000.0000 \mathrm{E}+000.0000 \mathrm{E}+000.0000 \mathrm{E}+000.0000 \mathrm{E}+000.00000 \mathrm{E}+000.0000 \mathrm{E}+00$ $0.0000 \mathrm{E}+000.0000 \mathrm{E}+000.0000 \mathrm{E}+000.0000 \mathrm{E}+(000.00000 \mathrm{E}+000.00060 \mathrm{E}+0.00 .0000 \mathrm{E}+00$ $0.0000 \mathrm{E}+000.0000 \mathrm{E}+000.0000 \mathrm{E}+000.0000 \mathrm{E}+00$

A Ground Water Flow Model for the A/M Area of the SRS (U)

$\mathrm{C} 2-2$ 
$0.0000 \mathrm{E}+000.0000 \mathrm{E}+000.0000 \mathrm{E}+000.0000 \mathrm{E}+000.0000 \mathrm{E}+000.0000 \mathrm{E}+000.0000 \mathrm{E}+00$ $0.00 \% 0 \mathrm{E}+000.0000 \mathrm{E}+000.0000 \mathrm{E}+0000.0000 \mathrm{E}+000.0000 \mathrm{E}+000.0000 \mathrm{E}+000.000(0) \mathrm{E}+00$ $0.0000 \mathrm{E}+000.0000 \mathrm{E}+000.00000 \mathrm{E}+(0) 0.0000 \mathrm{E}+000.0000 \mathrm{E}+000.0000) \mathrm{E}+(0) 0 .(0) 00 \mathrm{E}+00$ $0.0000 \mathrm{E}+000.0000 \mathrm{E}+000.00000 \mathrm{E}+000.0000 \mathrm{E}+00$

$0.0000 \mathrm{E}+000.00000 \mathrm{E}+000.0000 \mathrm{E}+000.0000 \mathrm{E}+000.0000 \mathrm{E}+000.000(0) \mathrm{E}+0 \times 0.00000 \mathrm{E}+00$ $0.0000 \mathrm{E}+000.0000 \mathrm{E}+000.0000 \mathrm{E}+000.0000 \mathrm{E}+000.0000 \mathrm{E}+000.0000 \mathrm{E}+000.0000 \mathrm{E}+00$ $0.0000 \mathrm{E}+000.0000 \mathrm{E}+000.0000 \mathrm{E}+000.0000 \mathrm{E}+000.0000 \mathrm{E}+000.0000 \mathrm{E}+000.0000 \mathrm{E}+00$ $0.0000 \mathrm{E}+0.00 .0000 \mathrm{E}+000.0000 \mathrm{E}+000.0000 \mathrm{E}+00$

$0.0000 \mathrm{E}+000.0000 \mathrm{E}+000.0000 \mathrm{E}+000.0000 \mathrm{E}+000.0000 \mathrm{E}+000.0000 \mathrm{E}+000.0000 \mathrm{E}+00$ $0.0000 \mathrm{E}+000.0000 \mathrm{E}+000.0000 \mathrm{E}+000.0000 \mathrm{E}+000.0000 \mathrm{E}+000.0000 \mathrm{E}+000.0000 \mathrm{E}+00$ $0.0000 \mathrm{E}+000.0000 \mathrm{E}+000.0000 \mathrm{E}+000.0000 \mathrm{E}+000.0000 \mathrm{E}+000.0000 \mathrm{E}+000.0000 \mathrm{E}+00$ $0.0000 \mathrm{E}+000.0000 \mathrm{E}+000.0000 \mathrm{E}+000.0000 \mathrm{E}+00$

$0.0000 \mathrm{E}+000.0000 \mathrm{E}+000.0000 \mathrm{E}+000.0000 \mathrm{E}+000.0000 \mathrm{E}+000.0000) \mathrm{E}+000.0000 \mathrm{E}+00$ $0.0000 \mathrm{E}+000.0000 \mathrm{E}+000.0000 \mathrm{E}+000.0000 \mathrm{E}+000.0000 \mathrm{E}+000.0000 \mathrm{E}+000,0000 \mathrm{E}+00$ $0.0000 \mathrm{E}+000.0000 \mathrm{E}+000.0000 \mathrm{E}+000.0000 \mathrm{E}+000.0000 \mathrm{E}+000.0000 \mathrm{E}+000.0000 \mathrm{E}+00$ $0.0000 \mathrm{E}+000.00000 \mathrm{E}+000.0000 \mathrm{E}+000.0000 \mathrm{E}+00$

$0.0000 \mathrm{E}+000.0000 \mathrm{E}+000.0000 \mathrm{E}+000.0000 \mathrm{E}+000.0000 \mathrm{E}+000.0000 \mathrm{E}+000.0000 \mathrm{E}+00$ $0.0000 \mathrm{E}+000.0000 \mathrm{E}+000.0000 \mathrm{E}+000.00000 \mathrm{E}+000.0000 \mathrm{E}+000.0000 \mathrm{E}+(000.0000 \mathrm{E}+00$ $0.0000 \mathrm{E}+000.0000 \mathrm{E}+000.0000 \mathrm{E}+000.0000 \mathrm{E}+00.9 .0000 \mathrm{E}+000.0000 \mathrm{E}+000.0000 \mathrm{E}+00$ $0.0000 \mathrm{E}+000.00000 \mathrm{E}+000.00000 \mathrm{E}+000.0000 \mathrm{E}+00$

$0.00000 \mathrm{E}+000.0000 \mathrm{E}+000.0000 \mathrm{E}+000.0000 \mathrm{E}+000.0000 \mathrm{E}+000.0000 \mathrm{E}+000.0000 \mathrm{E}+00$ $0.0000 \mathrm{E}+000.0000 \mathrm{E}+000.0000 \mathrm{E}+000.0000 \mathrm{E}+000.0000 \mathrm{E}+000.0000 \mathrm{E}+000.0000 \mathrm{E}+00$ $0.0000 \mathrm{E}+000.0000 \mathrm{E}+000.0000 \mathrm{E}+000.0000 \mathrm{E}+000.0000 \mathrm{E}+000.0000 \mathrm{E}+000.0000 \mathrm{E}+00$ $0.0000 \mathrm{E}+000.0000 \mathrm{E}+000.0000 \mathrm{E}+000.00000 \mathrm{E}+00$

$0.0000 \mathrm{E}+000.0000 \mathrm{E}+000.00000 \mathrm{E}+000.0000 \mathrm{E}+000.0000 \mathrm{E}+000.0000 \mathrm{E}+000.0000 \mathrm{E}+00$ $0.0000 \mathrm{E}+000.0000 \mathrm{E}+000.0000 \mathrm{E}+000.0000 \mathrm{E}+000.0000 \mathrm{E}+000.0000 \mathrm{E}+000.0000 \mathrm{E}+00)$ $0.0000 \mathrm{E}+000.0000 \mathrm{E}+000.0000 \mathrm{E}+000.0000 \mathrm{E}+000.0000 \mathrm{E}+000.0000 \mathrm{E}+000.0000 \mathrm{E}+00$ $0.0000 \mathrm{E}+000.0000 \mathrm{E}+000.0000 \mathrm{E}+0000.0000 \mathrm{E}+00$

$0.0000 \mathrm{E}+000.00000 \mathrm{E}+000.0000 \mathrm{E}+000.0000 \mathrm{E}+000.0000 \mathrm{E}+000.0000 \mathrm{E}+000.0000 \mathrm{E}+00$ $0.0000 \mathrm{E}+000.0000 \mathrm{E}+000.0000 \mathrm{E}+000.0000 \mathrm{E}+000.0000 \mathrm{E}+000.0000 \mathrm{E}+000.0000 \mathrm{E}+00$ $0.0000 \mathrm{E}+000.0000 \mathrm{E}+000.0000 \mathrm{E}+000.0000 \mathrm{E}+000.00000 \mathrm{E}+000.0000 \mathrm{E}+000.0000 \mathrm{E}+(00$ $0.0000 \mathrm{E}+000.00000 \mathrm{E}+000.0 .0000 \mathrm{E}+000.0000 \mathrm{E}+00$

$0.0000 \mathrm{E}+000.0000 \mathrm{E}+000.0000 \mathrm{E}+000.0000 \mathrm{E}+000.0000 \mathrm{E}+000.00000 \mathrm{E}+000.0000 \mathrm{E}+00$ $0.0000 \mathrm{E}+000.00000 \mathrm{E}+000.00000 \mathrm{E}+000.0000 \mathrm{E}+000.00000 \mathrm{E}+000.0000 \mathrm{E}+000.00000 \mathrm{E}+00$ $0.0000 \mathrm{E}+000.00000 \mathrm{E}+000.0000 \mathrm{E}+000.00000 \mathrm{E}+000.0000 \mathrm{E}+000.00000 \mathrm{E}+000.0000 \mathrm{E}+00$ $0.00000 \mathrm{E}+000.0000 \mathrm{E}+000.0000 \mathrm{E}+000.0000 \mathrm{E}+00$

$0.0000 \mathrm{E}+(00) 0.00000 \mathrm{E}+000.00000 \mathrm{E}+000.0000 \mathrm{E}+000.0000 \mathrm{E}+(000.0000 \mathrm{E}+000.0000 \mathrm{E}+00$ $0.00000 \mathrm{E}+00) 0.0(0) 0 \mathrm{E}+000.0000 \mathrm{E}+000.00000 \mathrm{E}+000.0000 \mathrm{E}+000.00000 \mathrm{E}+000.0000 \mathrm{E}+00$ $0.0000 \mathrm{E}+000.0000 \mathrm{E}+000.0000 \mathrm{E}+000.0000 \mathrm{E}+000.00000 \mathrm{E}+000.0000 \mathrm{E}+000.00000 \mathrm{E}+00$ $0.000(0) \mathrm{E}+000.0(0) 0 \mathrm{E}+000.0000 \mathrm{E}+000.0000 \mathrm{E}+(0)$



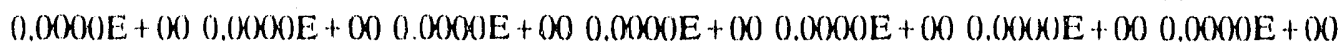
$0.000(0) \mathrm{E}+(00) 0.0000 \mathrm{E}+000.00000 \mathrm{E}+000.0000 \mathrm{E}+000.00000 \mathrm{E}+000.000(0) \mathrm{E}+000.00000 \mathrm{E}+00$

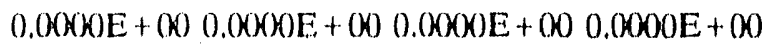

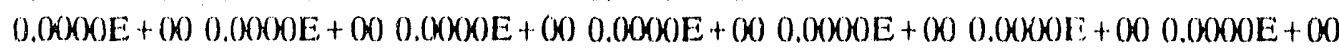
$0.00000 \mathrm{E}+000.00000 \mathrm{E}+000.00000 \mathrm{E}+000.0000 \mathrm{E}+000.00000 \mathrm{E}+000.0000 \mathrm{E}+000.000(0) \mathrm{E}+00$ $0.0000 \mathrm{E}+000.0000 \mathrm{E}+000.00000 \mathrm{E}+000.0000 \mathrm{E}+000.00000 \mathrm{E}+000.0000 \mathrm{E}+000.0000 \mathrm{E}+00$

A Ground Water Flow Model for the A/M Area of the SRS (U) 


\begin{tabular}{|c|c|c|c|c|c|c|}
\hline \\
\hline & & & & & & $0.00000 \mathrm{E}+000.0000 \mathrm{E}+000.0000 \mathrm{E}+000.0000 \mathrm{E}+000.0000 \mathrm{E}+000$. \\
\hline \multicolumn{7}{|c|}{$0.0000 \mathrm{E}+000.0000 \mathrm{E}+000.0000 \mathrm{E}+000.0000 \mathrm{E}+000.00000 \mathrm{E}+000}$. \\
\hline \multicolumn{7}{|c|}{$0.0000 \mathrm{E}+000.00000 \mathrm{E}+000.0000 \mathrm{E}+000.0000 \mathrm{E}+000.0000 \mathrm{E}+000$} \\
\hline \multicolumn{7}{|c|}{$0.0000 \mathrm{E}+000.0000 \mathrm{E}+000.00000 \mathrm{E}+000.0000 \mathrm{E}+00$} \\
\hline \multicolumn{7}{|c|}{$0.0000 \mathrm{E}+000.0000 \mathrm{E}+000.0000 \mathrm{E}+000.0000 \mathrm{E}+000.00000 \mathrm{E}+000.0$} \\
\hline \multicolumn{7}{|c|}{$0.0000 \mathrm{E}+000.0000 \mathrm{E}+000.00(0) \mathrm{E}+000.0000 \mathrm{E}+000.0(0) 0 \mathrm{E}+000.0$} \\
\hline \multicolumn{7}{|c|}{$0.0000 \mathrm{E}+000.0000 \mathrm{E}+000.0000 \mathrm{E}+000.0000 \mathrm{E}+000.0000 \mathrm{E}+000}$. \\
\hline \multicolumn{7}{|c|}{$0.0000 \mathrm{E}+000.0000 \mathrm{E}+000.0000 \mathrm{E}+000.0000 \mathrm{E}+0.0$} \\
\hline \multicolumn{4}{|c|}{$110.100 \mathrm{E}+01(7 \mathrm{G} 11.4)$} & & \multirow{2}{*}{1750.} \\
\hline 1750. & 1750. & 1750. & 1750. & 1750. & 1750. & \\
\hline 1750. & 1750. & 1750. & 1750. & 1750. & 1750. & 1750 \\
\hline 1750. & 1750. & 1750. & 1750. & 1750. & 1750. & 1750. \\
\hline 1750. & 1750. & 1750. & 1750. & & & \\
\hline 1750. & 1750. & 1750 . & 1750. & 1750. & 1750. & 1750 \\
\hline 1750. & 1750. & 1750. & 1750 . & 1750. & 1750. & 175 \\
\hline 1750. & 1750. & 1750. & 1750. & 1750. & 1750. & 1750. \\
\hline 1750 & $1^{\prime} / 50$ & 1750. & 1750 . & & & \\
\hline 1750. & $1: 50$. & 1750. & 1750) & 1750. & 1750. & 1750. \\
\hline 1750 & $175 ?$ ? & 1750 & 1750. & 1750 . & 1750. & 1750. \\
\hline 1750. & 1750. & 1750 & 1750 & 1750 . & 1750. & 1750. \\
\hline 1750. & 1750. & 1750 & 1750. & & & \\
\hline 1750. & 1750. & 1750 . & 1750 & 1750 . & 1750. & 1750. \\
\hline 1750. & 1750 & 1750 & 1750 & 1750. & $1^{\prime} 750$. & 1750 \\
\hline 1750. & 1750. & 1750 . & 1750. & 1750. & 1750. & 1750. \\
\hline 1750. & 1750. & 1750 & 1750. & & & \\
\hline 1750 . & 1750 & 1750 & 1750 & 1750. & 1750. & 1750. \\
\hline 1750. & 1750. & 1750 & 1750. & 1750 & 1750 . & 175 \\
\hline 1750 & 1750. & 1750. & 1750 & 1750 . & 1750. & 1750 . \\
\hline 1750. & 1750. & 1750 & 1750. & & & \\
\hline 1750. & 1750. & 1750 & 1750 & 1750 . & 1750. & 1750. \\
\hline 1750. & 1750. & 1750 & 1750. & 1750. & 1750. & 1750. \\
\hline 1750. & 1750 . & 1750 & 1750 & 1750 . & 1750. & 1750. \\
\hline 1750. & 1750 & 1750 & 1750. & & & \\
\hline 1750 & 1750 . & 1750. & 1750. & 1750 . & 1750 . & 175 \\
\hline 1750. & 1750. & 1750 & 1750 & 1750. & 175 & 175 \\
\hline 1750. & 1750 . & 1750 & 1750. & 1750 & 1750. & 1750 \\
\hline 1750. & 1750 . & 1750 & 1750 & & & \\
\hline 1750. & 1750 & 1750 . & 1750 & 1750 . & 1750. & 1750 \\
\hline 1750. & 1750 & 1750 & 1750 & 1750. & 1750 . & 1750 \\
\hline 1750. & 1750 & 1750 & 1750. & 950.0 & 950.0 & 1750. \\
\hline 1750. & 1750. & 1750 & 1750 & & & \\
\hline 1750. & 1750 & 1750 & 1750. & 1750. & 1750 & 1750. \\
\hline 1750. & 1750. & 1750 . & 1750 & 1750 . & 175 & 1750. \\
\hline 1750. & 1750 & 1750 & 1750. & 950.0 & 950.0 & 1750 \\
\hline 1750. & 1750 . & 1750 & 1750 . & & & \\
\hline 1750 & 1750 & 1750 & 1750. & 1750 & 1750. & 1750. \\
\hline
\end{tabular}




\begin{tabular}{|c|c|c|c|c|c|c|}
\hline 17.50 & 1750. & 1750. & 1750 & 1750. & 1750. & 1750. \\
\hline 1750. & 1750. & 1750 & 950,0 & 950.0 & 950.0 & 1750 \\
\hline 1750. & 1750. & 1750. & 1750 & & & \\
\hline 1750. & 1750. & 1750. & 1750 & 1750. & 1750 & 1750 \\
\hline 1750. & 1750 & 1750. & 1750 & 1750. & 1750 & 1750. \\
\hline 1750. & 950.0 & 950.0 & 950,0 & 950.0 & 1750 & 1750 \\
\hline 1750. & 1750 & 1750 & 1750 & & & \\
\hline 1750. & 1750 & 1750. & 1750. & 1750. & 1750. & 1750. \\
\hline 1750. & 1750. & 1750 & 1750 & 1750 & 1750 & 1750 \\
\hline 1750. & 950.0 & 950.0 & 950,0 & 950.0 & 1750 & 1750 \\
\hline 1750. & 1750. & 1750 & 1750 & & & \\
\hline 1750. & 1750. & 1750 & 1750 & 1750 & 1750 & 1750. \\
\hline 1750 & 1750. & 1750. & 1750 & 1750. & 1750 & 1750 \\
\hline 1750. & 950.0 & 950.0 & 950.0 & 950.0 & 1750 & \\
\hline 1750. & 1750. & 1750. & 1750 & & & \\
\hline 3100 & 3100 & 3100. & 3100 & 3100. & 3100. & 3100. \\
\hline 3100. & 3100. & 3100. & 3100. & 3100. & 3100 & 3100 \\
\hline 950.0 & 950.0 & 950.0 & 950.0 & 950.0 & & 1750. \\
\hline 1750. & 1750. & 1750. & 1750 & & & \\
\hline 3100. & 3100. & 3100. & 3100, & 3100. & 3100. & 3100. \\
\hline 3100. & 3100. & 3100 & 3100 & 3100. & 3100. & 3100. \\
\hline 950.0 & 950.0 & 950,0 & 950.0 & 950.0 & 1750 & 1750. \\
\hline 1750. & 1750. & 1750. & 1750 & & & \\
\hline 3100. & 3100. & 3100. & 3100 & 3100 & 3100. & 3100. \\
\hline 3100. & 3100 & 3100 & 3100 & 3100 & 3100. & 3100. \\
\hline 1750. & 1750. & 1750. & 1750 & & & \\
\hline 1750 & 1750. & 1750. & 1750. & & & \\
\hline 3100. & $31(0)$, & 3100 & 3100 & 3100 . & 3100. & 3100. \\
\hline 3100. & 3100. & 3100. & 3100. & 3100. & 3100. & 3100. \\
\hline 3100. & $31(00$. & $\begin{array}{l}3100 . \\
3100\end{array}$ & $\begin{array}{l}3100 . \\
3100\end{array}$ & & & $31(0)$. \\
\hline 3100. & $\begin{array}{l}31(0) . \\
3100 .\end{array}$ & 3100. & 3100 . & 3100. & 3100. & \\
\hline 3100. & $31(0)$. & 3100. & 3100. & 3100. & 3100. & 3100 , \\
\hline 3100. & $31(0)$, & 3100. & 3100. & 3100. & 3100 , & 3100. \\
\hline $31(0)$ & 3100. & 3100 & 3100. & & & \\
\hline 3100. & 3100. & 3100. & 3100 & $31(0)$ & 3100 & 3100. \\
\hline 3100. & $31(0)$. & 3100 & $31(0)$ & 3100 & $31(x)$ & 3100. \\
\hline 3100$)$ & 3100. & 3100. & $31(\mathrm{~K})$ & 3100 & 3100 & 3100. \\
\hline 3100. & 3100. & $31(x)$ & $31(k)$ & & & \\
\hline 3100. & 3100 . & 3100 & $31(0)$ & 3100 & $31(x)$ & $31(0)$ \\
\hline 3100. & 3100 & 3100. & 3100. & 3100 . & $31(x)$ & \\
\hline $\begin{array}{l}3100 . \\
3100)\end{array}$ & $\begin{array}{l}31(0) \\
3100(\end{array}$ & $\begin{array}{l}3100 . \\
3100 .\end{array}$ & $\begin{array}{l}31(0) \\
31(0)\end{array}$ & 3100 & 3100 & \\
\hline 3100$)$ & 3100. & 3100. & 3100. & 3100. & $31(0)$. & $31(x)$. \\
\hline 3100. & $31(x)$. & 3100. & 3100 & 3100 & 31000 & $31(k)$. \\
\hline 3100. & 3100. & 3100. & 3100. & 3100. & 3100. & 3100. \\
\hline
\end{tabular}




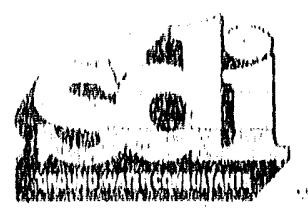

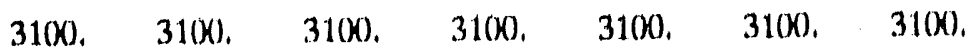

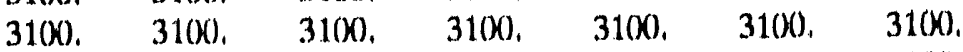
3100. 3100, 3100, 3100, 3100, 3100, 3100.

$\begin{array}{lllllll}3100, & 3100, & 3100, & 3100, & & & \\ 3100 & 3100, & 3100, & 3100, & 3100, & 3100, & 3100 .\end{array}$

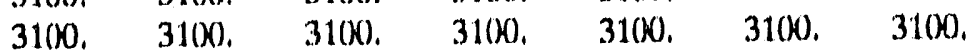
3100. 3100, 3100, 3100, 3100. 3100. 3100.

$\begin{array}{lllllll}3100, & 3100, & 3100, & 3100, & & & \\ 3100 . & 3100 . & 3100 . & 3100, & 3100, & 3100, & 3100 .\end{array}$

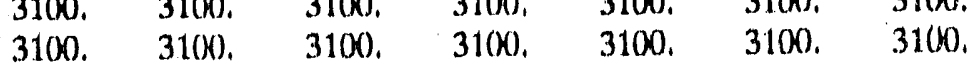

$3100.3100 .3100,3100,3100, \quad 3100 . \quad 3100$.

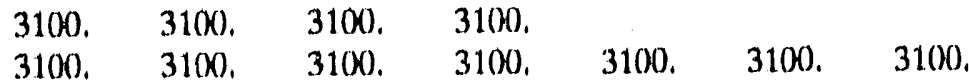

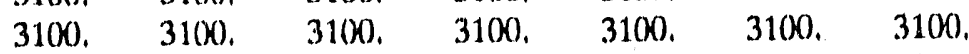

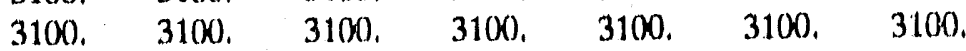

$\begin{array}{lllllll}3100, & 3100, & 3100, & 3100, & & & \\ 3100, & 3100, & 3100, & 3100 . & 3100, & 3100, & 3100 .\end{array}$

$3100.3100 .3100 . \quad 3100 . \quad 3100 . \quad 3100 . \quad 3100$.

3100. 3100, 3100, 3100. 3100. $3100 . \quad 3100$.

$\begin{array}{lllllll}3100, & 31(0) & 3100, & 3100, & & & \\ 3100, & 3100, & 3100, & 3100, & 3100, & 3100, & 3100 .\end{array}$

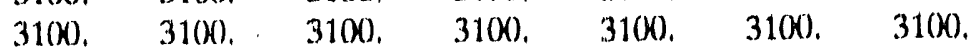

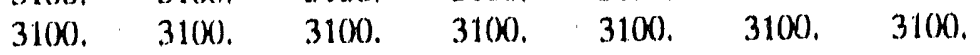

$\begin{array}{lllllll}31(0) & 31(0), & 3100, & 3100 . & & & \\ 3100 & 31(0), & 3100, & 3100, & 3100 . & 3100 . & 3100 .\end{array}$

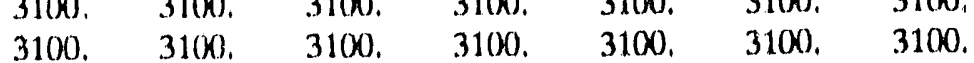

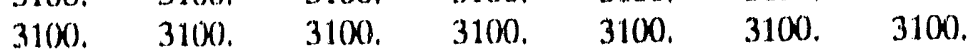

$\begin{array}{lllllll}3100 . & 31(0), & 3100 . & 3100 . & & & \\ 3100 . & 31(K), & 3100 . & 3100 . & 3100, & 3100 . & 3100 .\end{array}$

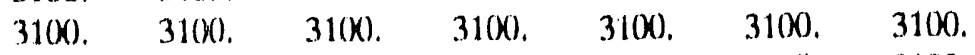

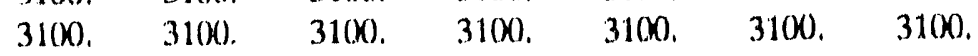

$\begin{array}{lllllll}3100 . & 3100 . & 3100 . & 31(0) . & & & \\ 3100 . & 3100 . & 3100 . & 3100 . & 3100 . & 3100 . & 3100 .\end{array}$

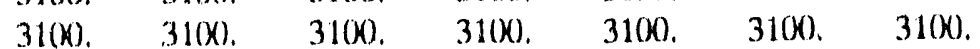

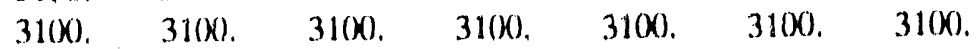

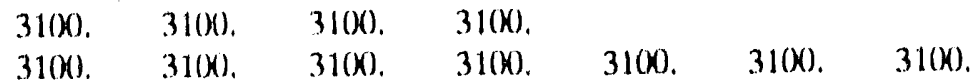

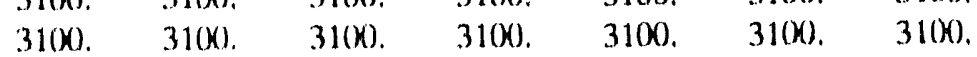

$3100.31(x) .3100 .3100 .3100 .3100 . \quad 3100$.

$\begin{array}{lllllll}3100 . & 31(x) . & 3100 . & 31(0) . & & & \\ 31(0) & 31(x) & 3100 . & 3100 . & 3100 . & 3100 . & 3100 .\end{array}$

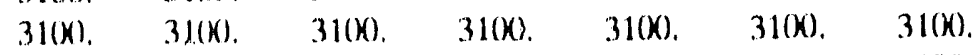

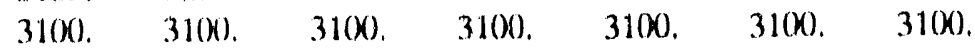

$\begin{array}{lllllll}31(0) & 31(K) . & 31(0) . & 31(K) . & & & \\ 3100 . & 31(x) . & 31(x) . & 31(K) . & 31(0) . & 3100 . & 31(K) .\end{array}$

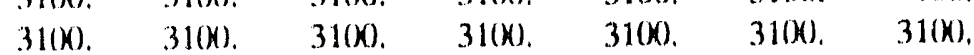

$31(0) .31(0), 3100.3100 .31(0), \quad 3100) .31(0)$. 


\begin{tabular}{|c|c|c|c|c|c|c|}
\hline 100. & $31(0)$ & 3100 & $31(x)$, & & & \\
\hline 3100. & $31(X)$ & $31(0)$ & $\begin{array}{l}31(0) \\
3100)\end{array}$ & $\begin{array}{l}31(0), \\
3100 .\end{array}$ & $\begin{array}{l}3100) \\
3100\end{array}$ & $\begin{array}{l}310 \\
310\end{array}$ \\
\hline $\begin{array}{l}(0), \\
(0) .\end{array}$ & $\begin{array}{l}3100 . \\
3100 .\end{array}$ & $\begin{array}{l}31(0) . \\
31(0) .\end{array}$ & $\begin{array}{l}3100 \%, \\
310 \%)\end{array}$ & 3100 . & 3100 & 310 \\
\hline 0 & $3100)$ & 3100. & $31(0)$. & & & \\
\hline 100. & 3100 , & 3100. & $31(k)$ & 3100. & 3100 & $10 x$ \\
\hline 100 & $31(0)$. & 3100, & 3100. & 310 & 311 & \\
\hline & 3100 , & 310() & 3100, & 3100. & 3100 . & 10 \\
\hline
\end{tabular}

$3100,3100.3100 .3100$.

$110.100 \mathrm{E}+01(7 \mathrm{G} 11.4)$

12

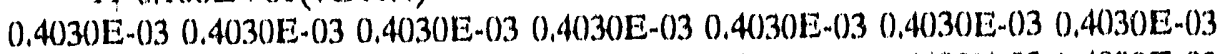
$0.4030 \mathrm{E}-(03) 0.4(130 \mathrm{E}-03 \quad 0.4(130 \mathrm{E}-0.3 \quad 0.4030 \mathrm{E}-03 \quad(0.4030 \mathrm{E}-03 \quad 0.4(130 \mathrm{E}-0.30 .4030 \mathrm{E}-03$ $0.4030 \mathrm{E}-03 \quad 0.4030 \mathrm{E}-03 \quad 0.4(130 \mathrm{E}-030.4030 \mathrm{E}-0.30 .4030 \mathrm{E}-(030,4030 \mathrm{E}-(03 \quad 0.4030 \mathrm{E}-03$ $0.7320 \mathrm{E}-010.7320 \mathrm{E}-(01 \quad 0.7320 \mathrm{E}-01 \quad 0.7320 \mathrm{E}-01$

$0.4030 \mathrm{E}-03 \quad 0.4030 \mathrm{E}-(03 \quad 0.4030 \mathrm{E}-03 \quad 0.4030 \mathrm{E}-03 \quad 0.4030 \mathrm{E}-03 \quad 0.4030 \mathrm{E}-03 \quad 0.4030 \mathrm{E}-03$ $0.4030 \mathrm{E}-(0.30 .4(030 \mathrm{E}-03 \quad 0.4030 \mathrm{E}-0.30 .4030 \mathrm{E}-03 \quad 0.4030 \mathrm{E}-03 \quad 0.4030 \mathrm{E}-(03 \quad 0.4030 \mathrm{E}-03$ $0.4030 \mathrm{E}-0.30 .4030 \mathrm{E}-03 \quad 0.4030 \mathrm{E}-0.30 .4030 \mathrm{E}-03 \quad 0.4030 \mathrm{E}-0.30 .4030 \mathrm{E}-03 \quad 0.4030 \mathrm{E}-03$ $0.7320 \mathrm{E}-(0)(0.7320 \mathrm{E}-010.7320 \mathrm{E}-(010.7320 \mathrm{E}-01$

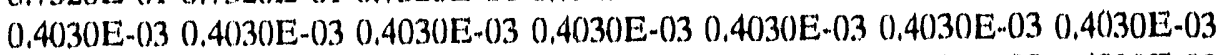

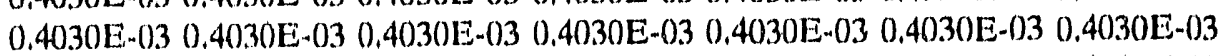
$0.4030 \mathrm{E}-03 \quad 0.4030 \mathrm{E}-(03 \quad 0.4030 \mathrm{E}-03 \quad 0.4030 \mathrm{E}-03 \quad 0.4030 \mathrm{E}-03 \quad 0.4030 \mathrm{E}-0.30 .4030 \mathrm{E}-03$ $0.7320 \mathrm{E}-010.7320 \mathrm{E} \cdot(010.732(0 \mathrm{E}-010.7320 \mathrm{E}-01$

$0.4030 \mathrm{E}-(0.30 .4030 \mathrm{E}-(03 \quad 0.40 .30 \mathrm{E}-(03 \quad 0.4030 \mathrm{E}-0.30 .4030 \mathrm{E}-03 \quad 0.4030 \mathrm{E}-0.30 .4030 \mathrm{E}-0.3$ $0.4(030 \mathrm{E}-(03)(0.4030 \mathrm{E}-03 \quad 0.4030 \mathrm{E}-03 \quad 0.4030 \mathrm{E}-03 \quad 0.4030 \mathrm{E}-03 \quad 0.4030 \mathrm{E}-03 \quad 0.4030 \mathrm{E}-03$ $0.4030 \mathrm{E}-03 \quad 0.4030 \mathrm{E}-03 \quad 0.4030 \mathrm{E}-0.3 \quad 0.4030 \mathrm{E}-03 \quad 0.40 .30 \mathrm{E}-03 \quad 0.4030 \mathrm{E}-(03 \quad 0.4030 \mathrm{E}-03$ $0.7320 \mathrm{E}-(010.7320 \mathrm{E}-010.7320 \mathrm{E}-010.7320 \mathrm{E}-01$

$0.4030 \mathrm{E}-03 \quad 0.4030 \mathrm{E}-03 \quad 0.4030 \mathrm{E}-03 \quad 0.4030 \mathrm{E}-03 \quad 0.40 .30 \mathrm{E}-03 \quad(0.4030 \mathrm{E}-030.4030 \mathrm{E}-03$ $0.4030 \mathrm{E}-0.30 .40 .30 \mathrm{E}-0.30 .4030 \mathrm{E}-03 \quad 0.4030 \mathrm{E}-0.330 .40 .30 \mathrm{E}-0.30 .4030 \mathrm{E}-(03) 0.1000 \mathrm{E}-03$ $0.1000 \mathrm{E}-0.30 .1000 \mathrm{E}-03 \quad 0.1000 \mathrm{E}-03 \quad 0.1000 \mathrm{E}-0330.1000 \mathrm{E}-03 \quad 0.1000 \mathrm{E}-0330.4030 \mathrm{E}-(0) 3$ $0.7320 \mathrm{E}-010.7320 \mathrm{E}-010.732(0 \mathrm{E}-010.7320 \mathrm{E}-01$

$0.4030 \mathrm{E}-(0.30 .4030 \mathrm{E}-03 \quad 0.4030 \mathrm{E}-0.30 .4030 \mathrm{E}-03 \quad 0.4030 \mathrm{E}-0.30 .4030 \mathrm{E}-(03 \quad 0.4030 \mathrm{E}-03$ $0.4030 \mathrm{E}-0330.4030 \mathrm{E}-03 \quad 0.4030 \mathrm{E}-0.30 .4030 \mathrm{E}-03 \quad 0.4030 \mathrm{E}-0.30 .4030 \mathrm{E}-03 \quad 0.1000 \mathrm{E}-0.3$

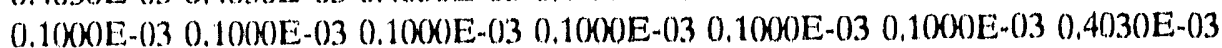
$0.732(0 \mathrm{E}-0) 10.7320 \mathrm{E}-010.7320 \mathrm{E}-0110.7320 \mathrm{E}-01$

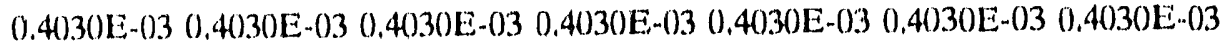



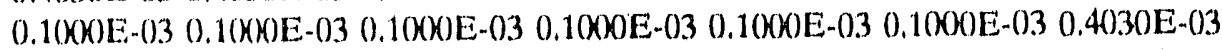
$0.7320 \mathrm{E} \cdot(010.7320 \mathrm{E}-010.7320 \mathrm{E}-010.7320 \mathrm{E}-01$

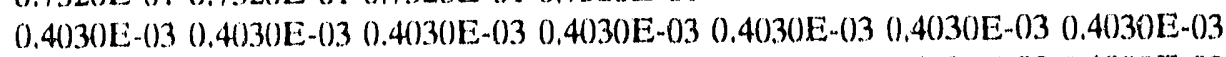

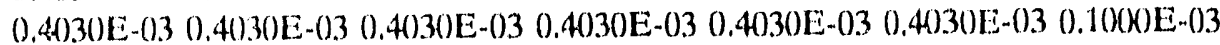

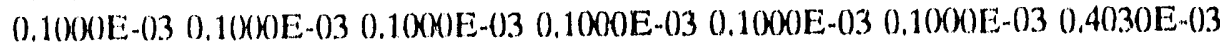
$0.7320 \mathrm{E}-(010.7320 \mathrm{E}-(01) .7320 \mathrm{E}-(010.7320 \mathrm{E}-01$

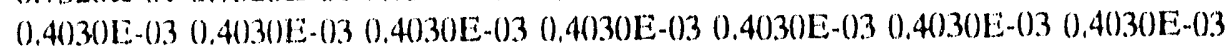

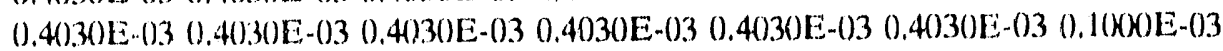

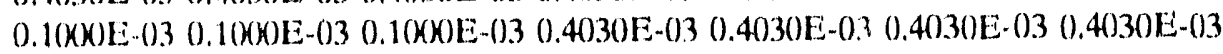
$0.7320 \mathrm{E}-(010.7320 \mathrm{E}-010.7320 \mathrm{E}-01 \quad 0.7320 \mathrm{E}-01$

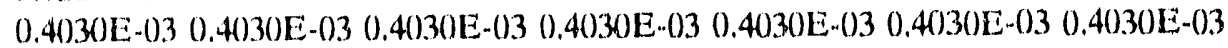

A Ground Watcr Flow Model for the A/M Arca of the SRS (U) 


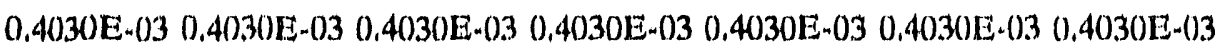
$0.4030 \mathrm{E}-(03 \quad 0.4030 \mathrm{E}-0.30 .40 .30 \mathrm{~F}-03 \quad 0.4030 \mathrm{E}-03 \quad 0.4030 \mathrm{E}-0.30 .4030 \mathrm{E}-0.30 .4030 \mathrm{E}-03$ $0.7320 \mathrm{E}-0110.7320 \mathrm{E}-010.7320 \mathrm{E}-(010.7320 \mathrm{E}-(01$

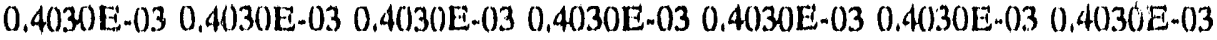

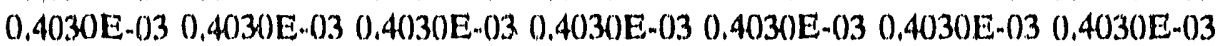
$0.4030 \mathrm{E}-(03 \quad 0.4030 \mathrm{E}-030.22(x) \quad 0.22(0) \quad 0.2200 \quad 0.4030 \mathrm{E}-03 \quad 0.4030 \mathrm{E} \cdot 0.3$ $0.7331 \mathrm{E}-010.7331 \mathrm{E}-010.7331 \mathrm{E}-010.7331 \mathrm{E}-01$ $0.4030 \mathrm{E}-(13) 0.4030 \mathrm{E}-0.30 .40) 30 \mathrm{E}-0.3 \quad 0.4030 \mathrm{E}-(130.4030 \mathrm{E}-0.3 \quad 0.4030 \mathrm{E}-(03 \quad 0.40330 \mathrm{E}-0.3$ $0.4030 \mathrm{E}-0.30 .4030 \mathrm{E}-03 \quad 0.4030 \mathrm{E}-03 \quad 0.4030 \mathrm{E}-03 \quad 0.4030 \mathrm{E}-03 \quad 0.4030 \mathrm{E}-(03 \quad 0.4030 \mathrm{E}-(13$

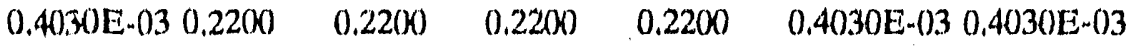
$0.7331 \mathrm{E}-(010.73331 \mathrm{E}-010.7331 \mathrm{E}-010.7331 \mathrm{E}-01$

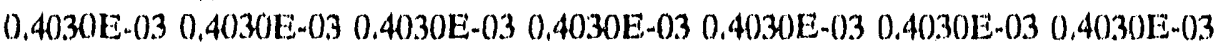
$0.4030 \mathrm{E}-0.3 \quad 0.4(0) 30 \mathrm{E}-03 \quad 0.4030 \mathrm{E}-0.3 \quad 0.4030 \mathrm{E}-03 \quad 0.4030 \mathrm{E}-0.3 \quad 0.4030 \mathrm{E}-03 \quad 0.4(130 \mathrm{E}-(03)$ $\begin{array}{lllllll}0.2200 & 0.2200 & 0.2200 & 0.2200 & 0.2200 & 0.2200 & 0.7330 \mathrm{E}-(01\end{array}$ $0.7331 \mathrm{E}-010.7331 \mathrm{E}-01 \quad 0.7331 \mathrm{E}-01 \quad 0.7331 \mathrm{E}-01$

$0.7330 \mathrm{E}-010.7330 \mathrm{E}-010.7330 \mathrm{E}-01 \quad 0.7330 \mathrm{E}-01 \quad 0.7330 \mathrm{E}-01 \quad 0.7330 \mathrm{E}-010.7330 \mathrm{E}-01$ $0.7330 \mathrm{E}-(010.7330 \mathrm{E}-(01 \quad 0.7330 \mathrm{E}-01 \quad(0.7330 \mathrm{E}-010.7330 \mathrm{E}-010.7330 \mathrm{E}-010.7330 \mathrm{E}-01$

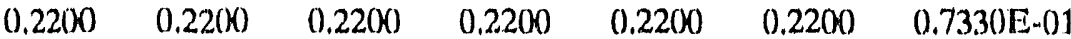
$0.7331 \mathrm{E}-010.7331 \mathrm{E}-010.7331 \mathrm{E}-010.7331 \mathrm{E}-01$

$0.7330 \mathrm{E}-010.7330 \mathrm{E}-010.7330 \mathrm{E}-010.7330 \mathrm{E}-01 \quad 0.73330 \mathrm{E}-(1) \quad 0.7330 \mathrm{E}-010.7330 \mathrm{E}-01$ $0.7330 \mathrm{E}-010.7330 \mathrm{E}-010.7330 \mathrm{E}-010.7330 \mathrm{E}-010.7330 \mathrm{E}-010.7330 \mathrm{E}-010.7330 \mathrm{E}-01$

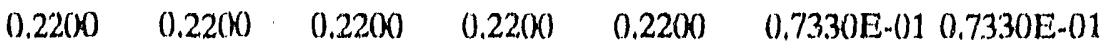
$0.7331 \mathrm{E}-010.7331 \mathrm{E}-010.7331 \mathrm{E}-01 \quad 0.7331 \mathrm{E}-01$

$0.7330 \mathrm{E}-(01 \quad 0.7330 \mathrm{E}-01 \quad 0.7330 \mathrm{E}-01 \quad 0.7330 \mathrm{E}-010.7330 \mathrm{E}-010.7330 \mathrm{E}-010.7330 \mathrm{E}-01$ $0.7330 \mathrm{E}-01 \quad 0.7330 \mathrm{E}-01 \quad 0.7330 \mathrm{E}-01 \quad 0.7330 \mathrm{E}-010.7330 \mathrm{E}-01 \quad 0.7330 \mathrm{E}-010.7330 \mathrm{E}-01$ $0.7330 \mathrm{E}-(01 \quad 0.7330 \mathrm{E}-(01 \quad 0.7330 \mathrm{E}-01 \quad 0.7330 \mathrm{E}-01 \quad 0.7330 \mathrm{E}-01 \quad 0.7330 \mathrm{E}-01 \quad 0.7330 \mathrm{E}-01$ $0.7330 \mathrm{E}-010.7330 \mathrm{E}-010.7330 \mathrm{E}-010.7330 \mathrm{E}-01$

$0.7330 \mathrm{E}-01 \quad 0.7330 \mathrm{E}-01 \quad 0.7330 \mathrm{E}-01 \quad 0.7330 \mathrm{E}-01 \quad 0.7330 \mathrm{E}-01 \quad 0.7330 \mathrm{E}-01 \quad 0.7330 \mathrm{E}-01$ 0.7330 E-01 0.7330 E-01 0.7330 E-01 0.7330 E-01 0.7330 E-01 0.7330 E-01 0.7330 E-01 $0.7330 \mathrm{E}-01 \quad 0.7330 \mathrm{E}-01 \quad 0.7330 \mathrm{E}-01 \quad 0.7330 \mathrm{E}-01 \quad 0.7330 \mathrm{E}-01 \quad 0.7330 \mathrm{E}-010.7330 \mathrm{E}-(01$ $0.7330 \mathrm{E}-01 \quad 0.7330 \mathrm{E}-01 \quad 0.7330 \mathrm{E}-010.7330 \mathrm{E}-01$

$0.7330 \mathrm{E}-01 \quad 0.7330 \mathrm{E}-01 \quad 0.7330 \mathrm{E}-010.7330 \mathrm{E}-01 \quad 0.7330 \mathrm{E}-010.7330 \mathrm{E}-010.7330 \mathrm{E}-01$ $0.7330 \mathrm{E}-01 \quad 0.7330 \mathrm{E}-01 \quad 0.7330 \mathrm{E}-(01 \quad 0.7330 \mathrm{E}-01 \quad 0.7330 \mathrm{E}-01 \quad 0.7330 \mathrm{E}-01 \quad 0.7330 \mathrm{E}-01$ $0.7330 \mathrm{E}-01 \quad 0.7330 \mathrm{E}-01 \quad 0.7330 \mathrm{E}-010.7330 \mathrm{E}-010.7330 \mathrm{E}-(01 \quad 0.7330 \mathrm{E}-010.7330 \mathrm{E}-01$ $0.7330 \mathrm{E}-010.7330 \mathrm{E}-01 \quad 0.7330 \mathrm{E}-01 \quad 0.7330 \mathrm{E}-01$

$0.7330 \mathrm{E}-01 \quad 0.7330 \mathrm{E}-01 \quad 0.7330 \mathrm{E}-01 \quad 0.7330 \mathrm{E}-01 \quad 0.7330 \mathrm{E}-01 \quad 0.7330 \mathrm{E}-01 \quad 0.7330 \mathrm{E}-01$ $0.7330 \mathrm{E}-0 \mathrm{i} \quad 0.7330 \mathrm{E}-01 \quad(0.7330 \mathrm{E}-01 \quad 0.7330 \mathrm{E}-01 \quad 0.7330 \mathrm{E}-01 \quad 0.7330 \mathrm{E}-(11 \quad 0.7330 \mathrm{E}-01$ $0.7330 \mathrm{E}-01 \quad 0.7330 \mathrm{E}-01 \quad 0.7330 \mathrm{E}-010.7330 \mathrm{E}-01 \quad 0.7330 \mathrm{E}-01 \quad 0.7330 \mathrm{E}-01 \quad 0.7330 \mathrm{E}-01$ $0.7330 \mathrm{E} \cdot 01 \quad 0.7330 \mathrm{E}-010.7330 \mathrm{E}-010.7330 \mathrm{E}-01$

$0.733(0 \mathrm{E}-(1) 10.7330 \mathrm{E}-01 \quad 0.7330 \mathrm{E}-01 \quad 0.7330 \mathrm{E}-01 \quad 0.7330 \mathrm{E}-010.733(0 \mathrm{E}-01 \quad 0.7330 \mathrm{E}-01$ $0.7330 \mathrm{E}-01 \quad 0.7330 \mathrm{E}-01 \quad 0.7330 \mathrm{E}-01 \quad 0.7330 \mathrm{E}-01 \quad 0.7330 \mathrm{E}-(01 \quad 0.7330 \mathrm{E}-01 \quad 0.7330 \mathrm{E}-01$ $0.7330 \mathrm{E}-010.7330 \mathrm{E}-01 \quad 0.7330 \mathrm{E}-01 \quad 0.7330 \mathrm{E}-01 \quad 0.7330 \mathrm{E}-01 \quad 0.7330 \mathrm{E}-010.7330 \mathrm{E}-01$ $0.7330 \mathrm{E}-010.7330 \mathrm{E}-010.7330 \mathrm{E}-01 \quad 0.7330 \mathrm{E}-01$

(0.7330E-01 $0.7330 \mathrm{E}-01 \quad 0.7330 \mathrm{E}-01 \quad 0.7330 \mathrm{E}-01 \quad 0.7330 \mathrm{E}-010.7330 \mathrm{E}-(01 \quad 0.7330 \mathrm{E}-01$ $0.7330 \mathrm{E}-010.7330 \mathrm{E}-01 \quad 0.7330 \mathrm{E}-01 \quad 0.7330 \mathrm{E}-01 \quad 0.7330 \mathrm{E}-010.7330 \mathrm{E}-(01 \quad 0.733 \mathrm{3}) \mathrm{E}-01$ $0.7330 \mathrm{E}-01 \quad 0.7330 \mathrm{E}-01 \quad 0.7330 \mathrm{E}-(01 \quad 0.7330 \mathrm{E}-010.7330 \mathrm{E}-(010.7330 \mathrm{E}-010.7330 \mathrm{E}-(01$ $0.7330 \mathrm{E}-01 \quad 0.7330 \mathrm{E}-01 \quad 0.7330 \mathrm{E}-01 \quad 0.7330 \mathrm{E}-01$ 


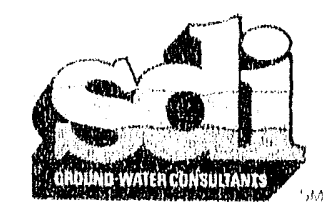

0.7330E-01 $0.7330 \mathrm{E}-01 \quad 0.7330 \mathrm{E}-01 \quad 0.7330 \mathrm{E}-01 \quad 0.7330 \mathrm{E}-01 \quad 0.7330 \mathrm{E}-01 \quad 0.7330 \mathrm{E}-01$ $0.7330 \mathrm{E}-01 \quad 0.7330 \mathrm{E}-01 \quad 0.7330 \mathrm{E}-01 \quad 0.7330 \mathrm{E}-01 \quad 0.7330 \mathrm{E}-01 \quad 0.7330 \mathrm{E}-010.7330 \mathrm{E}-01$

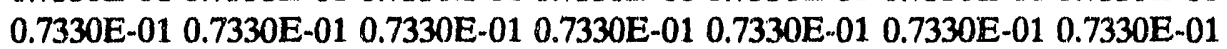
0.7330E-01 0.7330E-01 0.7330E-01 0.7330E-01

$0.7330 \mathrm{E}-01 \quad 0.7330 \mathrm{E}-\mathrm{C} 1 \quad 0.7330 \mathrm{E}-01 \quad 0.7330 \mathrm{E}-01 \quad 0.7330 \mathrm{E}-01 \quad 0.7330 \mathrm{E}-01 \quad 0.7330 \mathrm{E}-01$ $0.7330 \mathrm{E}-01 \quad 0.7330 \mathrm{E}-01 \quad 0.7330 \mathrm{E}-01 \quad 0.7330 \mathrm{E}-01 \quad 0.7330 \mathrm{E}-01 \quad 0.7330 \mathrm{E}-01 \quad 0.7330 \mathrm{E}-01$ 0.7330E-01 0.7330E-01 0.7330E-01 0.7330E-01 0.7330E-01 0.7330E-01 0.7330E-01 $0.7330 \mathrm{E}-010.7330 \mathrm{E}-010.7330 \mathrm{E}-010.7330 \mathrm{E}-01$

0.7330E-01 0.7330E-01 0.7330E-01 0.7330 E-01 0.7330 E-01 0.7330 E-01 0.7330 E-01 0.7330E-01 0.7330E-01 0.7330E-01 $0.7330 \mathrm{E}-01 \quad 0.7330 \mathrm{E}-01 \quad 0.7330 \mathrm{E}-01 \quad 0.733 \mathrm{CE}-01$ $0.7330 \mathrm{E}-01 \quad 0.7330 \mathrm{E}-01 \quad 0.7330 \mathrm{E}-01 \quad 0.7330 \mathrm{E}-01 \quad 0.7330 \mathrm{E}-01 \quad 0.7330 \mathrm{E}-010.7330 \mathrm{E}-01$ 0.7330E-01 0.7330E-01 0.7330E-01 0.7330E-01

0.7330 E-01 0.7330 E-01 0.7330 E-01 0.7330 E-01 0.7330 E-01 0.7330 E-01 0.7330 E-01 $0.7330 \mathrm{E}-01 \quad 0.7330 \mathrm{E}-01 \quad 0.7330 \mathrm{E}-01 \quad 0.7330 \mathrm{E}-01 \quad 0.7330 \mathrm{E}-01 \quad 0.7330 \mathrm{E}-01 \quad 0.7330 \mathrm{E}-01$ $0.7330 \mathrm{E}-01 \quad 0.7330 \mathrm{E}-01 \quad 0.7330 \mathrm{E}-01 \quad 0.7330 \mathrm{E}-01 \quad 0.7330 \mathrm{E}-01 \quad 0.7330 \mathrm{E}-01 \quad 0.7330 \mathrm{E}-01$ 0.7330E-01 0.7330E-01 0.7330E-01 0.7330E-01

$0.7330 \mathrm{E}-01 \quad 0.7330 \mathrm{E}-01 \quad 0.7330 \mathrm{E}-01 \quad 0.7330 \mathrm{E}-01 \quad 0.7330 \mathrm{E}-01 \quad 0.7330 \mathrm{E}-010.7330 \mathrm{E}-01$

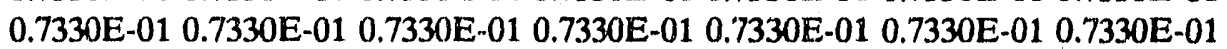
$0.7330 \mathrm{E}-01 \quad 0.7330 \mathrm{E}-01 \quad 0.7330 \mathrm{E}-01 \quad 0.7330 \mathrm{E}-01 \quad 0.7330 \mathrm{E}-01 \quad 0.7330 \mathrm{E}-01 \quad 0.7330 \mathrm{E}-01$ 0.7330E-01 $0.7330 \mathrm{E}-01 \quad 0.7330 \mathrm{E}-01 \quad 0.7330 \mathrm{E}-01$

0.7330 E-01 0.7330 E-01 0.7330E-01 0.7330 E-01 0.7330 E-01 0.7330 E-01 0.7330 E-01 $0.7330 \mathrm{E}-010.7330 \mathrm{E}-01 \quad 0.7330 \mathrm{E}-01 \quad 0.7330 \mathrm{E}-01 \quad 0.7330 \mathrm{E}-01 \quad 0.7330 \mathrm{E}-01 \quad 0.7330 \mathrm{E}-01$ 0.7330E-01 0.7330E-01 0.7330E-01 0.7330E-01 0.7330E-01 0.7330E-01 0.7330E-01 $0.7330 \mathrm{E}-010.7330 \mathrm{E}-01 \quad 0.7330 \mathrm{E}-01 \quad 0.7330 \mathrm{E}-01$

$\begin{array}{lllllll}0.7330 \mathrm{E}-01 & 0.7330 \mathrm{E}-01 & 0.7330 \mathrm{E}-01 & 0.7330 \mathrm{E}-01 & 0.7330 \mathrm{E}-01 & 0.7330 \mathrm{E}-01 & 0.7330 \mathrm{E}-01\end{array}$ 0.7330 E-01 0.7330 E-01 0.7330 E-01 0.7330 E-01 0.7330 E-01 0.7330 E-01 0.7330 E-01 $0.7330 \mathrm{E}-010.7330 \mathrm{E}-01 \quad 0.7330 \mathrm{E}-01 \quad 0.7330 \mathrm{E}-01 \quad 0.7330 \mathrm{E}-01 \quad 0.7330 \mathrm{E}-01 \quad 0.7330 \mathrm{E}-01$ $0.7330 \mathrm{E}-010.7330 \mathrm{E}-01 \quad 0.7330 \mathrm{E}-01 \quad 0.7330 \mathrm{E}-01$

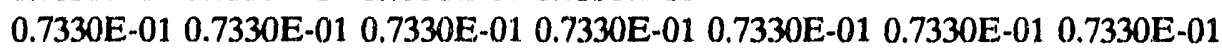
$0.7330 \mathrm{E}-010.7330 \mathrm{E}-01 \quad 0.7330 \mathrm{E}-01 \quad 0.7330 \mathrm{E}-01 \quad 0.7330 \mathrm{E}-010.7330 \mathrm{E}-01 \quad 0.7330 \mathrm{E}-01$ $0.7330 \mathrm{E}-01 \quad 0.7330 \mathrm{E}-01 \quad 0.7330 \mathrm{E}-01 \quad 0.7330 \mathrm{E}-01 \quad 0.7330 \mathrm{E}-01 \quad 0.7330 \mathrm{E}-01 \quad 0.7330 \mathrm{E}-01$ $0.7330 \mathrm{E}-01 \quad 0.7330 \mathrm{E}-01 \quad 0.7330 \mathrm{E}-01 \quad 0.7330 \mathrm{E}-01$

0.7330E-01 0.7330E-01 0.7330E-01 $0.7330 \mathrm{E}-01 \quad 0.7330 \mathrm{E}-01 \quad 0.7330 \mathrm{E}-01 \quad 0.7330 \mathrm{E}-01$ 0.7330 E-01 0.7330 E-01 0.7330 E-01 0.7330 E-01 0.7330 E-01 0.7330 E-01 0.7330 E-01

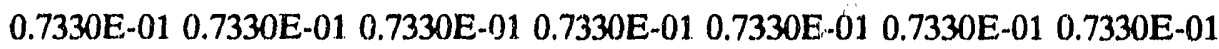
$0.7330 \mathrm{E}-01 \quad 0.7330 \mathrm{E}-01 \quad 0.7330 \mathrm{E}-01 \quad 0.7330 \mathrm{E}-01$

$0.7330 \mathrm{E}-01 \quad 0.7330 \mathrm{E}-01 \quad 0.7330 \mathrm{E}-01 \quad 0.7330 \mathrm{E}-01 \quad 0.7330 \mathrm{E}-01 \quad 0.7330 \mathrm{E}-01 \quad 0.7330 \mathrm{E}-01$

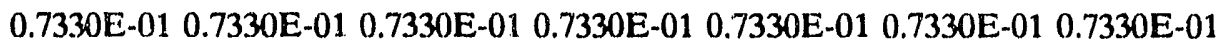
0.7330E-01 0.7330E-01 0.7330E-01 0.7330E-01 0.7330E-01 0.7330 E-01 0.7330 E-01 $0.7330 \mathrm{E}-010.7330 \mathrm{E}-010.7330 \mathrm{E}-010.7330 \mathrm{E}-01$

$0.7330 \mathrm{E}-01 \quad 0.7330 \mathrm{E}-01 \quad 0.7330 \mathrm{E}-01 \quad 0.7330 \mathrm{E}-01 \quad 0.7330 \mathrm{E}-010.7330 \mathrm{E}-01 \quad 0.7330 \mathrm{E}-01$ $0.7330 \mathrm{E}-01 \quad 0.7330 \mathrm{E}-01 \quad 0.7330 \mathrm{E}-01 \quad 0.7330 \mathrm{E}-01 \quad 0.7330 \mathrm{E}-01 \quad 0.7330 \mathrm{E}-01 \quad 0.7330 \mathrm{E}-01$ $\begin{array}{lllllll}0.7330 \mathrm{E}-01 & 0.7330 \mathrm{E}-01 & 0.7330 \mathrm{E}-01 & 0.7330 \mathrm{E}-01 & 0.7330 \mathrm{E}-01 & 0.7330 \mathrm{E}-01 & 0.7330 \mathrm{E}-01\end{array}$ $0.7330 \mathrm{E}-010.7330 \mathrm{E}-01 \quad 0.7330 \mathrm{E}-01 \quad 0.7330 \mathrm{E}-01$

$\begin{array}{lllllll}0.7330 \mathrm{E}-01 & 0.7330 \mathrm{E}-01 & 0.7330 \mathrm{E}-01 & 0.7330 \mathrm{E}-01 & 0.7330 \mathrm{E}-01 & 0.7330 \mathrm{E}-01 & 0.7330 \mathrm{E}-01\end{array}$ $\begin{array}{lllllll}0.7330 \mathrm{E}-01 & 0.7330 \mathrm{E}-01 & 0.7330 \mathrm{E}-01 & 0.7330 \mathrm{E}-01 & 0.7330 \mathrm{E}-01 & 0.7330 \mathrm{E}-01 & 0.7330 \mathrm{E}-01\end{array}$

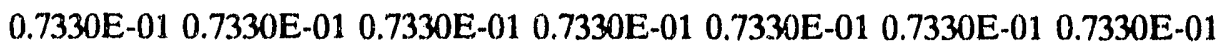

A Ground Water Flow Model for the A/M Area of the SRS (U)

C2-9 
0.7330E-01 0.7330E-01 0.7330E-01 0.7330E-01

$0.7330 \mathrm{E}-010.7330 \mathrm{E}-010.7330 \mathrm{E}-010.7330 \mathrm{E}-010.7330 \mathrm{E}-010.7330 \mathrm{E}-010.7330 \mathrm{E}-01$ $0.7330 \mathrm{E}-010.7330 \mathrm{E}-010.7330 \mathrm{E}-01 \quad 0.7330 \mathrm{E}-010.7330 \mathrm{E}-010.7330 \mathrm{E}-010.7330 \mathrm{E}-01$ $0.7330 \mathrm{E}-010.7330 \mathrm{E}-010.7330 \mathrm{E}-01 \quad 0.7330 \mathrm{E}-010.7330 \mathrm{E}-010.7330 \mathrm{E}-010.7330 \mathrm{E}-01$ $0.7330 \mathrm{E}-010.7330 \mathrm{E}-010.7330 \mathrm{E}-010.7330 \mathrm{E}-01$

0.7330E-01 0.7330E-01 0.7330E-01 0.7330E-01 0.7330E-01 0.7330E-01 0.7330E-01 $0.7330 \mathrm{E}-010.7330 \mathrm{E}-010.7330 \mathrm{E}-010.7330 \mathrm{E}-010.7330 \mathrm{E}-010.7330 \mathrm{E}-010.7330 \mathrm{E}-01$ $0.7330 \mathrm{E}-010.7330 \mathrm{E}-010.7330 \mathrm{E}-01 \quad 0.7330 \mathrm{E}-01 \quad 0.7330 \mathrm{E}-01 \quad 0.7330 \mathrm{E}-01 \quad 0.7330 \mathrm{E}-01$ $0.7330 \mathrm{E}-010.7330 \mathrm{E}-010.7330 \mathrm{E}-01 \quad 0.7330 \mathrm{E}-01$

\begin{tabular}{lllllll}
\multicolumn{1}{c}{11} & $\mathbf{8} 100 \mathrm{E}+01(7 \mathrm{G11.4})$ & \multicolumn{1}{c}{12} & & \\
880.0 & 880.0 & 880.0 & 880.0 & 880.0 & 880.0 & 880.0 \\
880.0 & 880.0 & 880.0 & 880.0 & 880.0 & 880.0 & 880.0 \\
880.0 & 880.0 & 880.0 & 880.0 & 880.0 & 880.0 & 880.0 \\
880.0 & 880.0 & 880.0 & 880.0 & & & \\
880.0 & 880.0 & 880.0 & 880.0 & 880.0 & 880.0 & 880.0 \\
880.0 & 880.0 & 880.0 & 880.0 & 880.0 & 880.0 & 880.0 \\
880.0 & 880.0 & 880.0 & 880.0 & 880.0 & 880.0 & 880.0 \\
880.0 & 880.0 & 880.0 & 880.0 & & & \\
880.0 & 880.0 & 880.0 & 880.0 & 880.0 & 880.0 & 880.0 \\
880.0 & 880.0 & 880.0 & 880.0 & 880.0 & 880.0 & 880.0 \\
880.0 & 880.0 & 880.0 & 880.0 & 880.0 & 880.0 & 880.0 \\
880.0 & 880.0 & 880.0 & 880.0 & & & \\
880.0 & 880.0 & 880.0 & 880.0 & 880.0 & 880.0 & 880.0 \\
880.0 & 880.0 & 880.0 & 880.0 & 880.0 & 880.0 & 880.0 \\
880.0 & 880.0 & 880.0 & 880.0 & 880.0 & 880.0 & 880.0 \\
880.0 & 880.0 & 880.0 & 880.0 & & & \\
880.0 & 880.0 & 880.0 & 880.0 & 880.0 & 880.0 & 880.0 \\
880.0 & 880.0 & 880.0 & 880.0 & 880.0 & 880.0 & 880.0 \\
880.0 & 880.0 & 880.0 & 880.0 & 880.0 & 880.0 & 880.0 \\
880.0 & 880.0 & 880.0 & 880.0 & & & \\
880.0 & 880.0 & 880.0 & 880.0 & 880.0 & 880.0 & 880.0 \\
880.0 & 880.0 & 880.0 & 880.0 & 880.0 & 880.0 & 880.0 \\
880.0 & 880.0 & 880.0 & 880.0 & 880.0 & 880.0 & 880.0 \\
880.0 & 880.0 & 880.0 & 880.0 & & & \\
880.0 & 880.0 & 880.0 & 880.0 & 880.0 & 880.0 & 880.0 \\
880.0 & 880.0 & 880.0 & 880.0 & 880.0 & 880.0 & 880.0 \\
880.0 & 880.0 & 880.0 & 880.0 & 880.0 & 880.0 & 880.0 \\
880.0 & 880.0 & 880.0 & 880.0 & & & \\
880.0 & 880.0 & 880.0 & 880.0 & 880.0 & 880.0 & 880.0 \\
880.0 & 880.0 & 880.0 & 880.0 & 880.0 & 880.0 & 880.0 \\
880.0 & 880.0 & 880.0 & 880.0 & 450.0 & 450.0 & 880.0 \\
880.0 & 880.0 & 880.0 & 880.0 & & & \\
880.0 & 880.0 & 880.0 & 880.0 & 880.0 & 880.0 & 880.0 \\
880.0 & 880.0 & 880.0 & 880.0 & 880.0 & 880.0 & 880.0 \\
880.0 & 880.0 & 880.0 & 880.0 & 450.0 & 450.0 & 880.0 \\
880.0 & 880.0 & 880.0 & 880.0 & & & \\
880.0 & 880.0 & 880.0 & 880.0 & 880.0 & 880.0 & 880.0
\end{tabular}




$\begin{array}{lllllll}880.0 & 880.0 & 880.0 & 880.0 & 880.0 & 880.0 & 880.0 \\ 880.0 & 880.0 & 880.0 & 450.0 & 450.0 & 450.0 & 880.0 \\ 880.0 & 880.0 & 880.0 & 880.0 & & & \\ 880.0 & 880.0 & 880.0 & 880.0 & 880.0 & 880.0 & 880.0 \\ 880.0 & 880.0 & 880.0 & 880.0 & 880.0 & 880.0 & 880.0 \\ 880.0 & 450.0 & 450.0 & 450.0 & 450.0 & 880.0 & 880.0 \\ 880.0 & 880.0 & 880.0 & 880.0 & & & \\ 880.0 & 880.0 & 880.0 & 880.0 & 880.0 & 880.0 & 880.0 \\ 880.0 & 880.0 & 880.0 & 880.0 & 880.0 & 880.0 & 880.0 \\ 880.0 & 450.0 & 450.0 & 450.0 & 450.0 & 880.0 & 880.0 \\ 880.0 & 880.0 & 880.0 & 880.0 & & & \\ 2400 . & 2400 . & 2400 . & 2400 . & 2400 . & 2400 . & 2400 . \\ 2400 . & 2400 . & 2400 . & 2400 . & 2400 . & 2400 . & 2400 . \\ 2400 . & 450.0 & 450.0 & 450.0 & 450.0 & 2400 . & 2400 . \\ 2400 . & 2400 . & 2400 . & 2400 . & & & \\ 2400 . & 2400 . & 2400 . & 2400 . & 2400 . & 2400 . & 2400 . \\ 2400 . & 2400 . & 2400 . & 2400 . & 2400 . & 2400 . & 2400 . \\ 450.0 & 450.0 & 450.0 & 450.0 & 450.0 & 2400 . & 2400 . \\ 2400 . & 2400 . & 2400 . & 2400 . & & & \\ 2400 . & 2400 . & 2400 . & 2400 . & 2400 . & 2400 . & 2400 . \\ 2400 . & 2400 . & 2400 . & 2400 . & 2400 . & 2400 . & 2400 . \\ 450.0 & 450.0 & 450.0 & 450.0 & 450.0 & 2400 . & 2400 . \\ 2400 . & 2400 . & 2400 . & 2400 . & & & \\ 2400 . & 2400 . & 2400 . & 2400 . & 2400 . & 2400 . & 2400 . \\ 2400 . & 2400 . & 2400 . & 2400 . & 2400 . & 2400 . & 2400 . \\ 2400 . & 2400 . & 2400 . & 2400 . & 2400 . & 2400 . & 2400 . \\ 2400 . & 2400 . & 2400 . & 2400 . & & & \\ 2400 . & 2400 . & 2400 . & 2400 . & 2400 . & 2400 . & 2400 . \\ 2400 . & 2400 . & 2400 . & 2400 . & 2400 . & 2400 . & 2400 . \\ 2400 . & 2400 . & 2400 . & 2400 . & 2400 . & 2400 . & 2400 . \\ 2400 . & 2400 . & 2400 . & 2400 . & & & \\ 2400 . & 2400 . & 2400 . & 2400 . & 2400 . & 2400 . & 2400 . \\ 2400 . & 2400 . & 2400 . & 2400 . & 2400 . & 2400 . & 2400 . \\ 2400 . & 2400 . & 2400 . & 2400 . & 2400 . & 2400 . & 2400 . \\ 2400 . & 2400 . & 2400 . & 2400 . & & & \\ 2400 . & 2400 . & 2400 . & 2400 . & 2400 . & 2400 . & 2400 . \\ 2400 . & 2400 . & 2400 . & 2400 . & 2400 . & 2400 . & 2400 . \\ 2400 . & 2400 . & 2400 . & 2400 . & 2400 . & 2400 . & 2400 . \\ 2400 . & 2400 . & 2400 . & 2400 . & & & \\ 2400 . & 2400 . & 2400 . & 2400 . & 2400 . & 2400 . & 2400 . \\ 2400 . & 2400 . & 2400 . & 2400 . & 2400 . & 2400 . & 2400 . \\ 2400 . & 2400 . & 2400 . & 2400 . & 2400 . & 2400 . & 2400 . \\ 2400 . & 2400 . & 2400 . & 2400 . & & & \\ 2400 . & 2400 . & 2400 . & 2400 . & 2400 . & 2400 . & 2400 . \\ 2400 . & 2400 . & 2400 . & 2400 . & 2400 . & 2400 . & 2400 . \\ 2400 . & 2400 . & 2400 . & 2400 . & 2400 . & 2400 . & 2400 . \\ 2400 . & 2400 . & 2400 . & 2400 . & & & \\ & & & & & & \end{array}$




$\begin{array}{lllllll}2400 . & 2400 . & 2400 . & 2400 . & 2400 . & 2400 . & 2400 . \\ 2400 . & 2400 . & 2400 . & 2400 . & 2400 . & 2400 . & 2400 . \\ 2400 . & 2400 . & 2400 . & 2400 . & 2400 . & 2400 . & 2400 . \\ 2400 . & 2400 . & 2400 . & 2400 . & & & \\ 2400 . & 2400 . & 2400 . & 2400 . & 2400 . & 2400 . & 2400 . \\ 2400 . & 2400 . & 2400 . & 2400 . & 2400 . & 2400 . & 2400 \\ 2400 . & 2400 . & 2400 . & 2400 . & 2400 . & 2400 . & 2400 . \\ 2400 . & 2400 . & 2400 . & 2400 . & & & \\ 2400 . & 2400 . & 2400 . & 2400 . & 2400 . & 2400 . & 2400 . \\ 2400 . & 2400 . & 2400 . & 2400 . & 2400 . & 2400 . & 2400 . \\ 2400 . & 2400 . & 2400 . & 2400 . & 2400 . & 2400 . & 2400 . \\ 2400 . & 2400 . & 2400 . & 2400 . & & & \\ 2400 . & 2400 . & 2400 . & 2400 . & 2400 . & 2400 . & 2400 . \\ 2400 . & 2400 . & 2400 . & 2400 . & 2400 . & 2400 . & 2400 . \\ 2400 . & 2400 . & 2400 . & 2400 . & 2400 . & 2400 . & 2400 . \\ 2400 . & 2400 . & 2400 . & 2400 . & & & \\ 2400 . & 2400 . & 2400 . & 2400 . & 2400 . & 2400 . & 2400 . \\ 2400 . & 2400 . & 2400 . & 2400 . & 2400 . & 2400 . & 2400 . \\ 2400 . & 2400 . & 2400 . & 2400 . & 2400 . & 2400 . & 2400 . \\ 2400 . & 2400 . & 2400 . & 2400 . & & & \\ 2400 . & 2400 . & 2400 . & 2400 . & 2400 . & 2400 . & 2400 . \\ 2400 . & 2400 . & 2400 . & 2400 . & 2400 . & 2400 . & 2400 . \\ 2400 . & 2400 . & 2400 . & 2400 . & 2400 . & 2400 . & 2400 . \\ 2400 . & 2400 . & 2400 . & 2400 . & & & \\ 2400 . & 2400 . & 2400 . & 2400 . & 2400 . & 2400 . & 2400 . \\ 2400 . & 2400 . & 2400 . & 2400 . & 2400 . & 2400 . & 2400 . \\ 2400 . & 2400 . & 2400 . & 2400 . & 2400 . & 2400 . & 2400 . \\ 2400 . & 2400 . & 2400 . & 2400 . & & & \\ 2400 . & 2400 . & 2400 . & 2400 . & 2400 . & 2400 . & 2400 . \\ 2400 . & 2400 . & 2400 . & 2400 . & 2400 . & 2400 . & 2400 . \\ 2400 . & 2400 . & 2400 . & 2400 . & 2400 . & 2400 . & 2400 . \\ 2400 . & 2400 . & 2400 . & 2400 . & & & \\ 2400 . & 2400 . & 2400 . & 2400 . & 2400 . & 2400 . & 2400 . \\ 2400 . & 2400 . & 2400 . & 2400 . & 2400 . & 2400 . & 2400 . \\ 2400 . & 2400 . & 2400 . & 2400 . & 2400 . & 2400 . & 2400 . \\ 2400 . & 2400 . & 2400 . & 2400 . & & & \\ 2400 . & 2400 . & 2400 . & 2400 . & 2400 . & 2400 . & 2400 . \\ 2400 . & 2400 . & 2400 . & 2400 . & 2400 . & 2400 . & 2400 . \\ 2400 . & 2400 . & 2400 . & 2400 . & 2400 . & 2400 . & 2400 . \\ 2400 . & 2400 . & 2400 . & 2400 . & & & \\ 2400 . & 2400 . & 2400 . & 2400 . & 2400 . & 2400 . & 2400 . \\ 2400 . & 2400 . & 2400 . & 2400 . & 2400 . & 2400 . & 2400 . \\ 2400 . & 2400 . & 2400 . & 2400 . & 2400 . & 2400 . & 2400 . \\ 2400 . & 2400 . & 2400 . & 2400 . & & & \\ 2400 . & 2400 . & 2400 . & 2400 . & 2400 . & 2400 . & 2400 . \\ 2400 . & 2400 . & 2400 . & 2400 . & 2400 . & 2400 . & 2400 . \\ 2400 . & 2400 . & 2400 . & 2400 . & 2400 . & 2400 . & 2400 . \\ & & & & & & \end{array}$


2400 . 2400. 2400 . 2400 .

2400 . 2400. 2400 . 2400.2400 . 2400 . 2400 .

2400 . 2400. 2400.2400 . 2400. 2400 . 2400 .

2400 . 2400. $2400.2400 .2400 .240 J .2400$.

$\begin{array}{lllllll}2400 . & 2400 . & 2400 . & 2400 . & & & \\ 2400 . & 2400 . & 2400 . & 2400 . & 2400 . & 2400 . & 2400 .\end{array}$

2400 . 2400. 2400. 2400. 2400. 2400 . 2400 .

2400 . 2400. 2400. 2400. 2400. 2400. 2400 .

2400 . 2400. 2400 . 2400 .

$110.100 \mathrm{E}+01(7 \mathrm{G} 11.4)$

12

$0.4000 \mathrm{E}-04$ 0.4000E-04 0.4000E-04 0.4000E-04 0.4000E-04 0.4000E-04 0.4000E-04

$0.4000 \mathrm{E}-04 \quad 0.2000 \mathrm{E}-050.2000 \mathrm{E}-05 \quad 0.2000 \mathrm{E}-050.2000 \mathrm{E}-050.2000 \mathrm{E}-050.2000 \mathrm{E}-05$ 0.2000 E-05 $0.2000 \mathrm{E}-050.2000 \mathrm{E}-050.2000 \mathrm{E}-05$ 0.4000E-04 0.5500E-03 0.5500E-03 $0.5500 \mathrm{E}-03 \quad 0.5500 \mathrm{E}-03 \quad 0.5500 \mathrm{E}-03 \quad 0.5500 \mathrm{E}-03$

$0.4000 \mathrm{E}-04 \quad 0.4000 \mathrm{E}-04$ 0.4000E-04 0.4000E-04 0.4000E-04 0.4000E-04 0.4ن00E-04 $0.4000 \mathrm{E}-04 \quad 0.2000 \mathrm{E}-050.2000 \mathrm{E}-050.2000 \mathrm{E}-05 \quad 0.2000 \mathrm{E}-05 \quad 0.2000 \mathrm{E}-050.2000 \mathrm{E}-05$ $0.2000 \mathrm{E}-050.2000 \mathrm{E}-050.2000 \mathrm{E}-050.2000 \mathrm{E}-050.4000 \mathrm{E}-04$ 0.5500E-03 0.5500E-03 $0.5500 \mathrm{E}-03 \quad 0.5500 \mathrm{E}-03 \quad 0.5500 \mathrm{E}-03 \quad 0.5500 \mathrm{E}-03$

$0.4000 \mathrm{E}-040.4000 \mathrm{E}-04$ 0.4000E-04 0.4000E-04 0.4000E-04 0.4000E-04 0.4000E-04 $0.4000 \mathrm{E}-04 \quad 0.2000 \mathrm{E}-050.2000 \mathrm{E}-050.2000 \mathrm{E}-050.2000 \mathrm{E}-050.2000 \mathrm{E}-050.2000 \mathrm{E}-05$ $0.2000 \mathrm{E}-05 \quad 0.2000 \mathrm{E}-050.2000 \mathrm{E}-050.2000 \mathrm{E}-050.4000 \mathrm{E}-04 \quad 0.5500 \mathrm{E}-03 \quad 0.5500 \mathrm{E}-03$ $0.5500 \mathrm{E}-03 \quad 0.5500 \mathrm{E}-03 \quad 0.5500 \mathrm{E}-030.5500 \mathrm{E}-03$

$0.4000 \mathrm{E}-04 \quad 0.4000 \mathrm{E}-04 \quad 0.4000 \mathrm{E}-04 \quad 0.4000 \mathrm{E}-04 \quad 0.4000 \mathrm{E}-04 \quad 0.4000 \mathrm{E}-040.4000 \mathrm{E}-04$ $0.4000 \mathrm{E}-04 \quad 0.2000 \mathrm{E}-05 \quad 0.200 \mathrm{CE}-050.2000 \mathrm{E}-050.2000 \mathrm{E}-050.2000 \mathrm{E}-050.2000 \mathrm{E}-05$ $0.2000 \mathrm{E}-050.2000 \mathrm{E}-050.2000 \mathrm{E}-050.2000 \mathrm{E}-050.4000 \mathrm{E}-040.5500 \mathrm{E}-03 \quad 0.5500 \mathrm{E}-03$ $0.5500 \mathrm{E}-03 \quad 0.5500 \mathrm{E}-03 \quad 0.5500 \mathrm{E}-03 \quad 0.5500 \mathrm{E}-03$

$0.4000 \mathrm{E}-04$ 0.4000E-04 0.4000E-04 0.4000E-04 0.4000E-04 0.4000E-04 0.4000E-04 $0.4000 \mathrm{E}-04 \quad 0.2000 \mathrm{E}-0.50 .2000 \mathrm{E}-050.2000 \mathrm{E}-050.2000 \mathrm{E}-050.2000 \mathrm{E}-050.2000 \mathrm{E}-05$ 0.2000 E-05 $0.2000 \mathrm{E}-050.2000 \mathrm{E}-050.2000 \mathrm{E}-050.4000 \mathrm{E}-040.5500 \mathrm{E}-03 \quad 0.5500 \mathrm{E}-03$ $0.5500 \mathrm{E}-03 \quad 0.5500 \mathrm{E}-03 \quad 0.5500 \mathrm{E}-03 \quad 0.5500 \mathrm{E}-03$

$0.4000 \mathrm{E}-040.4000 \mathrm{E}-040.4000 \mathrm{E}-04$ 0.4000E-04 0.4000E-04 0.4000E-04 0.4000E-04 $0.4000 \mathrm{E}-040.2000 \mathrm{E}-05 \quad 0.2000 \mathrm{E}-0.50 .2000 \mathrm{E}-05 \quad 0.2000 \mathrm{E}-050.2000 \mathrm{E}-050.2000 \mathrm{E}-0.5$ $0.2000 \mathrm{E}-050.2000 \mathrm{E}-050.2000 \mathrm{E}-050.2000 \mathrm{E}-050.4000 \mathrm{E}-040.5500 \mathrm{E}-03 \quad 0.5500 \mathrm{E}-03$ $0.5500 \mathrm{E}-03 \quad 0.5500 \mathrm{E}-03 \quad 0.5500 \mathrm{E}-03 \quad 0.5500 \mathrm{E}-03$

$0.4000 \mathrm{E}-04$ 0.4000E-04 0.4000E-04 0.4000E-04 0.4000E-04 0.4000E-04 0.4000E-04 $0.4000 \mathrm{E}-04 \quad 0.2000 \mathrm{E}-05 \quad 0.2000 \mathrm{E}-05 \quad 0.2000 \mathrm{E}-05 \quad 0.2000 \mathrm{E}-05 \quad 0.2000 \mathrm{E}-05 \quad 0.2000 \mathrm{E}-05$ $0.2000 \mathrm{E}-050.2000 \mathrm{E}-050.2000 \mathrm{E}-0.50 .2000 \mathrm{E}-050.4000 \mathrm{E}-040.5500 \mathrm{E}-03 \quad 0.5500 \mathrm{E}-03$ $0.5500 \mathrm{E}-030.5500 \mathrm{E}-03 \quad 0.5500 \mathrm{E}-03 \quad 0.5500 \mathrm{E}-03$

$0.4000 \mathrm{E}-040.4000 \mathrm{E}-040.4000 \mathrm{E}-040.4000 \mathrm{E}-040.4000 \mathrm{E}-040.4000 \mathrm{E}-040.4000 \mathrm{E}-04$ $0.4000 \mathrm{E}-040.2000 \mathrm{E}-0.50 .2000 \mathrm{E}-050.2000 \mathrm{E}-050.2000 \mathrm{E}-050.2000 \mathrm{E}-050.2000 \mathrm{E}-05$ $0.2000 \mathrm{E}-050.2000 \mathrm{E}-050.2000 \mathrm{E}-050.2000 \mathrm{E}-0.50 .4000 \mathrm{E}-(040.5500 \mathrm{E}-030.5500 \mathrm{E}-03$ $0.5500 \mathrm{E}-03 \quad 0.5500 \mathrm{E}-03 \quad 0.5500 \mathrm{E}-030.5500 \mathrm{E}-03$

$0.4000 \mathrm{E}-040.4000 \mathrm{E}-040.4000 \mathrm{E}-040.4000 \mathrm{E}-040.4000) \mathrm{E}-04(1.4000 \mathrm{E}-040.4000 \mathrm{E}-04$ $0.4000 \mathrm{E}-040.2000 \mathrm{E}-050.2000 \mathrm{E}-050.2000 \mathrm{E}-050.2000 \mathrm{E}-0.50 .2000 \mathrm{E}-0.50 .2000 \mathrm{E}-0.5$ $0.2000 \mathrm{E}$ 25 0.2000E-05 $0.2000 \mathrm{E}-050.2000 \mathrm{E}-050.4000 \mathrm{E}-040.5500 \mathrm{E}-030.5500 \mathrm{E}-03$ $0.5500 \mathrm{E}-03 \quad 0.5500 \mathrm{E}-030.5500 \mathrm{E}-030.5500 \mathrm{E}-03$

0.4000E-04 0.4000E-04 0.4000E-04 0.4000E-04 0.4000E-04 0.4000E-04 0.4090E-04 


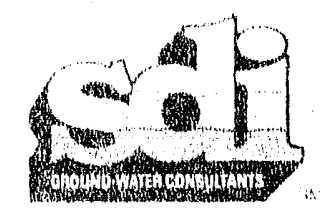

0.4000E-04 0.4000E-04 0.4000E-04 0.4000E-04 0.2000E-05 0.2000E-05 0.2000E-05 $0.2000 \mathrm{E}-05$ 0.2000E-05 0.2000E-05 0.2000E-05 0.4000E-04 0.5500E-03 0.5500E-03 $0.5500 \mathrm{E}-03 \quad 0.5500 \mathrm{E}-03 \quad 0.5500 \mathrm{E}-03 \quad 0.5500 \mathrm{E}-03$

0.4000E-04 0.4000E-04 0.4000E-04 0.4000E-04 0.4000E-04 0.4000E-04 0.4000E-04 0.4000E-04 0.4000E-04 0.4000E-04 0.4000E-04 0.2000E-05 0.2000E-05 0.2000E-0.5 0.2000E-05 0.2000E-05 0.2000E-05 0.2000E-05 0.4000E-04 0.4000E-04 0.4000E-04 $0.4000 \mathrm{E}-040.5500 \mathrm{E}-03 \quad 0.5500 \mathrm{E}-03 \quad 0.5500 \mathrm{E}-03$

0.4000E-04 0.4000E-04 0.4000E-04 0.4000E-04 0.4000E-04 0.4000E-04 0.4000E-04 0.4000E-04 0.4000E-04 0.4000E-04 0.4000E-04 0.2000E-05 0.2000E-05 0.2000E-05 0.4000E-04 0.4000E-04 0.4000E-04 0.4000E-04 0.4030E-04 0.4000E-04 0.4030E-04 $0.4000 \mathrm{E}-040.5500 \mathrm{E}-03 \quad 0.5500 \mathrm{E}-03 \quad 0.5500 \mathrm{E}-03$

0.4000E-04 0.4000E-04 0.4000E-04 0.4000E-04 0.4000E-04 0.4000E-04 0.4000E-04 $0.4000 \mathrm{E}-04 \quad 0.4000 \mathrm{E}-040.4000 \mathrm{E}-04 \quad 0.4000 \mathrm{E}-04$ 0.2000E-05 0.2000E-05 0.4000E-04 $0.4000 \mathrm{E}-040.4000 \mathrm{E}-04$ 0.4000E-04 0.4000E-04 0.2000E-05 0.2000E-05 0.2000E-05 0.4000E-04 0.4000E-04 0.4000E-04 0.4000E-04

0.4000E-04 0.4000E-04 0.4000E-04 0.4000E-04 0.4000E-04 0.4000E-04 0.4000E-04 $0.4000 \mathrm{E}-04 \quad 0.4000 \mathrm{E}-04 \quad 0.4000 \mathrm{E}-04 \quad 0.4000 \mathrm{E}-04 \quad 0.2000 \mathrm{E}-05 \quad 0.4000 \mathrm{E}-04 \quad 0.4000 \mathrm{E}-04$ 0.4000 E-04 0.4000E-04 0.4000E-04 0.2000E-05 0.2000E-05 0.2000E-05 0.2000E-05 $0.4000 \mathrm{E}-040.4000 \mathrm{E}-040.4000 \mathrm{E}-040.4000 \mathrm{E}-04$

0.4000E-04 0.4000E-04 0.4000E-04 0.4000E-04 0.4000E-04 0.4000E-04 0.4000E-04 $0.4000 \mathrm{E}-040.4000 \mathrm{E}-04$ 0.4000E-04 0.4000E-04 0.4000E-04 0.4000E-04 0.4000E-04 $0.4000 \mathrm{E}-04 \quad 0.4000 \mathrm{E}-04 \quad 0.2000 \mathrm{E}-05 \quad 0.2000 \mathrm{E}-05$ 0.2000E-05 0.2000E-05 0.2000E-05 $0.2000 \mathrm{E}-050.4000 \mathrm{E}-04$ 0.4000E-04 0.4000E-04

0.4000E-04 0.4000E-04 0.4000E-04 0.4000E-04 0.4000E-04 0.4000E-04 0.4000E-04 $0.4000 \mathrm{E}-040.4000 \mathrm{E}-040.4000 \mathrm{E}-040.4000 \mathrm{E}-040.4000 \mathrm{E}-040.4000 \mathrm{E}-040.4000 \mathrm{E}-04$ $0.4000 \mathrm{E}-04 \quad 0.4000 \mathrm{E}-040.2000 \mathrm{E}-050.2000 \mathrm{E}-05$ 0.2000E-05 0.2000E-05 0.2000E-05 0.2000E-05 0.4000E-04 0.4000E-04 0.4000E-04

0.4000E-04 0.4000E-04 0.4000E-04 0.4000E-04 0.4000E-04 0.4000E-04 0.4000E-04 0.4000E-04 0.4000E-04 0.4000E-04 0.4000E-04 0.4000E-04 0.4000E-04 0.4000E-04 $0.4000 \mathrm{E}-04$ 0.4000E-04 0.2000E-05 0.2000E-05 0.2000E-05 0.2000E-05 0.2000E-05 $0.2000 \mathrm{E}-05$ 0.2000E-05 0.4000E-04 0.4000E-04

$0.4000 \mathrm{E}-04 \quad 0.4000 \mathrm{E}-040.4000 \mathrm{E}-04 \quad 0.4000 \mathrm{E}-04 \quad 0.4000 \mathrm{E}-040.4000 \mathrm{E}-04 \quad 0.4000 \mathrm{E}-04$ 0.4000E-04 0.4000E-04 0.4000E-04 0.4000E-04 0.4000E-01 0.4000E-04 0.4000E-04 0.4000E-04 0.4000E-(04 0.2000E-05 0.2000E-05 0.2000E-05 0.2000E-05 0.2000E-0.5 $0.2000 \mathrm{E}-050.2000 \mathrm{E}-050.4000 \mathrm{E}-040.4000 \mathrm{E}-04$

0.4000E-04 0.4000E-04 0.4000E-04 0.4000E-04 0.4000E-04 0.4000E-04 0.4000E-04 $0.4000 \mathrm{E}-040.4000 \mathrm{E}-04 \quad 0.4000 \mathrm{E}-04 \quad 0.4000 \mathrm{E}-04$ 0.4000E-04 0.4000E-04 0.4000E-04 $0.4000 \mathrm{E}-040.4000 \mathrm{E}-040.4000 \mathrm{E}-04 \quad 0.4000 \mathrm{E}-04 \quad 0.4000 \mathrm{E}-04 \quad 0.4000 \mathrm{E}-04$ 0.4000E-04 $0.4000 \mathrm{E}-040.4000 \mathrm{E}-040.4000 \mathrm{E}-040.4000 \mathrm{E}-04$

0.4000E-04 0.4000E-04 0.4000E-04 0.4000E-04 0.4000E-04 0.4000E-04 0.4000E - 04 0.4000E-04 0.4000E-04 0.4000E-04 0.4000E-04 0.4000E-04 0.4000E-04 0.4000E0.4000E-04 0.4000E-04 0.4000E-04 0.4000E-04 0.4000E-04 0.4000E-04 0.4000E-04 $0.4000 \mathrm{E}-040.4000 \mathrm{E}-04$ 0.4000E-04 0.4000E-04

$0.4000 \mathrm{E}-040.4000 \mathrm{E}-040.4000 \mathrm{E}-040.4000 \mathrm{E}-040.4000 \mathrm{E}-(04) 0.4000 \mathrm{E}-040.4000 \mathrm{E}-04$ $0.4000 \mathrm{E}-040.4000 \mathrm{E}-040.4000 \mathrm{E}-04 \quad 0.4000 \mathrm{E}-040.4000 \mathrm{E}-04 \quad 0.4000 \mathrm{E}-040.4000 \mathrm{E}-04$ $0.4000 \mathrm{E}-04 \quad 0.4000 \mathrm{E}-04 \quad 0.4000 \mathrm{E}-040.4000 \mathrm{E}-04$ 0.4000 E-04 0.4000E-04 0.4000E-04 $0.4000 \mathrm{E}-040.4000 \mathrm{E}-040.40(n) \mathrm{E}-040.4000 \mathrm{E}-04$

A Ground Water Flow Model for the A/M Area of the SRS (U)

C2-14 


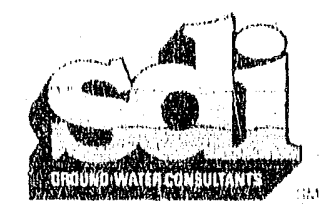

0.4000E-04 0.4000E-04 0.4000E-04 0.4000E-04 0.4000E-04 0.4000E-04 0.4000E-04 0.4000E-04 0.4000E-04 0.4000E-04 0.4000E-04 0.4000E-04 0.4000E-04 0.4000E-04 0.4000E-04 0.4000E-04 0.4000E-04 0.4000E-04 0.4000E-04 0.4000E-04 0.4000E-04 $0.4000 \mathrm{E}-040.4000 \mathrm{E}-040.4000 \mathrm{E}-040.4000 \mathrm{E}-04$

0.4000E-04 0.4000E-04 0.4000E-04 0.4000E-04 0.4000E-04 0.4000E-04 0.4000E-04 0.4000E-04 0.4000E-04 0.4000E-04 0.4000E-04 0.4000E-04 0.4000E-04 0.4000E-04 0.4000E-04 0.4000E-04 0.4000E-04 0.4000E-04 0.4000E-04 0.4000E-04 0.4000E-04 $0.4000 \mathrm{E}-040.4000 \mathrm{E}-040.4000 \mathrm{E}-040.4000 \mathrm{E}-04$

0.4000E-04 0.4000E-04 0.4000E-04 0.4000E-04 0.4000E-04 0.4000E-04 0.4000E-04 0.4000E-04 0.4000E-04 0.4000E-04 0.4000E-04 0.4000E-04 0.4000E-04 0.4000E-04 0.4000E-04 0.4000E-04 0.4000E-04 0.4000E-04 0.4000E-04 0.4000E-04 0.4000E-04 $0.4000 \mathrm{E}-040.4000 \mathrm{E}-04 \quad 0.4000 \mathrm{E}-040.4000 \mathrm{E}-04$

0.4000E-04 0.4000E-04 0.4000E-04 0.4000E-04 0.4000E-04 0.4000E-04 0.4000E-04 0.4000E-04 0.4000E-04 0.4000E-04 0.4000E-04 0.4000E-04 0.4000E-04 0.4000E-04 0.4000E-04 0.4000E-04 0.4000E-04 0.4000E-04 0.4000E-04 0.4000E-04 0.4000E-04 $0.4000 \mathrm{E}-040.4000 \mathrm{E}-040.4000 \mathrm{E}-040.4000 \mathrm{E}-04$

$0.4000 \mathrm{E}-04 \quad 0.4000 \mathrm{E}-04 \quad 0.4000 \mathrm{E}-04 \quad 0.4000 \mathrm{E}-04 \quad 0.4000 \mathrm{E}-040.4000 \mathrm{E}-04 \quad 0.4000 \mathrm{E}-04$ 0.4000E-04 0.4000E-04 0.4000E-04 0.4000E-04 0.4000E-04 0.4000E-04 0.4000E-04 0.4000E-04 0.4000E-04 0.4000E-04 0.4000E-04 0.4000E-04 0.4000E-04 0.4000E-04 0.4000E-04 0.4000E-04 0.4000E-04 0.4000E-04

$0.4000 \mathrm{E}-04 \quad 0.4000 \mathrm{E}-04 \quad 0.4000 \mathrm{E}-04 \quad 0.4000 \mathrm{E}-04$ 0.4000E-04 0.4000E-04 0.4000E-04 $0.4000 \mathrm{E}-040.4000 \mathrm{E}-04 \quad 0.4000 \mathrm{E}-04 \quad 0.4000 \mathrm{E}-04 \quad 0.4000 \mathrm{E}-04 \quad 0.4000 \mathrm{E}-040.4000 \mathrm{E}-04$ $0.4000 \mathrm{E}-040.4000 \mathrm{E}-040.4000 \mathrm{E}-040.4000 \mathrm{E}-04$ 0.4000E-04 0.4000E-04 0.4000E-04 $0.4000 \mathrm{E}-040.4000 \mathrm{E}-040.4000 \mathrm{E}-040.4000 \mathrm{E}-04$

0.4000E-04 0.4000E-04 0.4000E-04 0.4000E-04 0.4000E-04 0.4000E-04 0.4000E-04 0.4000E-04 0.4000E-04 0.4000E-04 0.4000E-04 0.4000E-04 0.4000E-04 0.4000E-04 $0.4000 \mathrm{E}-040.4000 \mathrm{E}-04 \quad 0.4000 \mathrm{E}-04 \quad 0.4000 \mathrm{E}-040.4000 \mathrm{E}-04 \quad 0.4000 \mathrm{E}-040.4000 \mathrm{E}-04$ $0.4000 \mathrm{E}-040.4000 \mathrm{E}-040.4000 \mathrm{E}-040.4000 \mathrm{E}-04$

$0.4000 \mathrm{E}-040.4000 \mathrm{E}-04 \quad 0.4000 \mathrm{E}-04 \quad 0.4000 \mathrm{E}-04 \quad 0.4000 \mathrm{E}-040.4000 \mathrm{E}-04 \quad 0.4000 \mathrm{E}-04$ $0.4000 \mathrm{E}-040.4000 \mathrm{E}-040.4000 \mathrm{E}-040.4000 \mathrm{E}-04$ 0.4000E-04 0.4000E-04 0.4000E-04 $0.4000 \mathrm{E}-04 \quad 0.4000 \mathrm{E}-04 \quad 0.4000 \mathrm{E}-04 \quad 0.4000 \mathrm{E}-04$ 0.4000E-04 0.4000E-04 0.4000E-04 $0.4000 \mathrm{E}-040.4000 \mathrm{E}-040.4000 \mathrm{E}-040.4000 \mathrm{E}-04$

$0.4000 \mathrm{E}-040.4000 \mathrm{E}-04 \quad 0.4000 \mathrm{E}-04 \quad 0.4000 \mathrm{E}-04$ 0.4000E-04 0.4000E-(-64 0.4000E-04 $0.4000 \mathrm{E}-040.4000 \mathrm{E}-04$ 0.4000E-04 0.4000E-04 0.4000E-04 0.4000E-04 0.4000E-04 0.4000E-04 0.4000E-04 0.4000E-04 0.4000E-04 0.4000E-04 0.4000E-04 0.4000E-04 $0.4000 \mathrm{E}-040.4000 \mathrm{E}-040.4000 \mathrm{E}-040.4000 \mathrm{E}-04$

0.4000E-04 0.4000E-04 0.4000E-04 0.4000E-04 0.4000E-04 0.4000E-04 0.4000E-04 $0.4000 \mathrm{E}-04 \quad 0.4000 \mathrm{E}-04 \quad 0.4000 \mathrm{E} .04 \quad 0.4000 \mathrm{E}-04 \quad 0.4000 \mathrm{E}-04 \quad 0.4000 \mathrm{E}-04 \quad 0.4000 \mathrm{E}-04$ 0.4000 E-04 0.4000E-04 0.4000E-04 0.4000E-04 0.4000E-04 0.4000E-04 0.4000E-04 $0.4000 \mathrm{E}-040.4000 \mathrm{E}-04 \quad 0.4000 \mathrm{E}-040.4000 \mathrm{E}-04$

0.4000E-04 0.4000E-04 0.4000E-04 0.4000E-04 0.4000E-04 0.4000E-04 0.4000E-04 0.4000 E-04 0.4000E-04 0.4000E-04 0.4000E-04 0.4000E-04 0.4000E-04 0.4000E-04 $0.4000 \mathrm{E}-040.4000 \mathrm{E}-040.4000 \mathrm{E}-04 \quad 0.4000 \mathrm{E}-040.4000 \mathrm{E}-040.4000 \mathrm{E}-040.4000 \mathrm{E}-04$ $0.4000 \mathrm{E}-040.4000 \mathrm{E}-040.4000 \mathrm{E}-040.4000 \mathrm{E}-04$

0.4000E-04 0.4000E-04 0.4000E-04 0.4000)E-04 0.4000E-04 0.4000E-04 0.4000E-04 $0.4000 \mathrm{E}-0 .+0.4000 \mathrm{E}-040.4000 \mathrm{E}-04$ 0.4000E-04 0.4000E-04 0.4000E-04 0.4000E-(14 0.4000E-1)4 0.4000E-04 0.4000E-04 0.4000E-04 0.4000E-04 0.4000E-04 0.4000E-04

A Ground Water Flow Model for the A/M Area of the SRS (U)

C2-15 
0.4000E-04 0.4000E-04 0.4000E-04 0.4000E-04

$0.4000 \mathrm{E}-04 \quad 0.4000 \mathrm{E}-04 \quad 0.4000 \mathrm{E}-04 \quad 0.4000 \mathrm{E}-04 \quad 0.4000 \mathrm{E}-040.4000 \mathrm{E}-040.4000 \mathrm{E}-04$ $0.4000 \mathrm{E}-040.4000 \mathrm{E}-040.4000 \mathrm{E}-04 \quad 0.4000 \mathrm{E}-040.4000 \mathrm{E}-04 \quad 0.4000 \mathrm{E}-040.4000 \mathrm{E}-04$ 0.4000 E-04 0.4000E-04 0.4000E-04 0.4000E-04 0.4000E-04 0.4000E-04 0.4000E-04 0.4000E-04 0.400CE-04 0.4000E-04 0.4000E-04

$0.4000 \mathrm{E}-040.4000 \mathrm{E}-040.4000 \mathrm{E}-040.4000 \mathrm{E}-040.4000 \mathrm{E}-040.4000 \mathrm{E}-040.4000 \mathrm{E}-04$ $0.4000 \mathrm{E}-040.4000 \mathrm{E}-04$ 0.4000E-04 0.4000E-04 0.4000E-04 0.4000E-04 0.4000E-04 $0.4000 \mathrm{E}-040.4000 \mathrm{E}-04$ 0.4000E-04 0.4000E-04 0.4000E-04 0.4000E-04 0.4000E-04 $0.4000 \mathrm{E}-040.4000 \mathrm{E}-040.4000 \mathrm{E}-040.4000 \mathrm{E}-04$

$0.400 \mathrm{E}+04$ 
APPENDIX C3

Well Package 
WSSC.WEL

$\begin{array}{rrl}33 & 0 & \\ 33 & & \\ 4 & 8 & 17-.4493 \mathrm{E}+05 \\ 4 & 9 & 17-.2566 \mathrm{E}+05 \\ 4 & 26 & 10-4813 . \\ 1 & 10 & 16-260.0 \\ 2 & 10 & 16-3292 . \\ 3 & 10 & 16-1646 . \\ 1 & 8 & 16-231.0 \\ 2 & 8 & 16-2926 . \\ 3 & 8 & 16-1463 . \\ 1 & 8 & 17-1502 . \\ 2 & 8 & 17-.1902 \mathrm{E}+05 \\ 3 & 8 & 17-9509 . \\ 1 & 9 & 16-404.0 \\ 2 & 9 & 16-5121 . \\ 3 & 9 & 16-2560 . \\ 1 & 9 & 17-48.00 \\ 2 & 9 & 17-610.0 \\ 3 & 9 & 17-305.0 \\ 1 & 11 & 17-481.0 \\ 2 & 11 & 17-6096 . \\ 3 & 11 & 17-3048 . \\ 1 & 11 & 16-279.0 \\ 2 & 11 & 16-3536 . \\ 3 & 11 & 16-1768 . \\ 1 & 11 & 14-327.0 \\ 2 & 11 & 14-4145 . \\ 3 & 11 & 14-2072 . \\ 1 & 11 & 15-578.0 \\ 2 & 11 & 15-7315 . \\ 3 & 11 & 15-3658 \\ 4 & 13 & 25-.3209 \mathrm{E}+05 \\ 4 & 13 & 24-.3208 \mathrm{E}+05 \\ 4 & 6 & 17-1925 . \\ & & \\ & \\ 3\end{array}$


APPENDIX CA

General Head Boundary Package 
WSSC.GHB

\begin{tabular}{|c|c|c|}
\hline 82 & -1 & \\
\hline & & \\
\hline 2 & 6 & $10.150 E+030.500 E+04$ \\
\hline 0 & 7 & $10.150 \mathrm{E}+030.500 \mathrm{E}+04$ \\
\hline 2 & 8 & $10.150 \mathrm{E}+030.500 \mathrm{E}+04$ \\
\hline 2 & 9 & 1. $0.150 \mathrm{E}+030.500 \mathrm{E}+04$ \\
\hline 2 & 10 & $10.150 E+030.500 E+04$ \\
\hline O & 11 & $10.150 E+030.500 E+04$ \\
\hline 2 & 21 & $250.195 \mathrm{E}+0.30 .100 \mathrm{E}+05$ \\
\hline 2 & 22 & $240.190 \mathrm{E}+030.100 \mathrm{E}+05$ \\
\hline 2 & 13 & $200.213 E+030.100 E+05$ \\
\hline 2 & 14 & $200.211 E+030.100 E+05$ \\
\hline 2 & 15 & $190.206 \mathrm{E}+030.100 \mathrm{E}+05$ \\
\hline 2 & 16 & $190.204 E+030.100 E+05$ \\
\hline 2 & 17 & $190.201 E+030.100 E+05$ \\
\hline 2 & 18 & $180.195 E+030.100 E+05$ \\
\hline 2 & 19 & $180.188 E+030.100 E+05$ \\
\hline 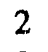 & 20 & $180.182 \mathrm{E}+030.100 \mathrm{E}+05$ \\
\hline 2 & 21 & $170.175 E+030.100 E+05$ \\
\hline 2 & 22 & $170.168 \mathrm{E}+030.100 \mathrm{E}+05$ \\
\hline 2 & 12 & $10.150 \mathrm{E}+03 \quad 0.500 \mathrm{E}+04$ \\
\hline 2 & 13 & $10.150 \mathrm{E}+030.500 \mathrm{E}+04$ \\
\hline 2 & 14 & 1. $0.150 \mathrm{E}+030.5(\mathrm{YOE}+04$ \\
\hline 2 & 15 & $10.150 \mathrm{E}+030.500 \mathrm{E}+04$ \\
\hline 2 & 16 & $10.150 E+030.500 E+04$ \\
\hline 2 & 17 & $10.150 \mathrm{E}+030.500 \mathrm{E}+04$ \\
\hline 2 & 13 & $10.150 \mathrm{E}+030.500 \mathrm{E}+04$ \\
\hline 2 & 19 & $10.150 \mathrm{E}+030.500 \mathrm{E}+04$ \\
\hline 2 & 20 & $10.150 E+030.500 E+04$ \\
\hline 2 & 21 & $10.150 \mathrm{E}+030.500 \mathrm{E}+04$ \\
\hline 2 & 22 & $10.150 \mathrm{E}+030.500 \mathrm{E}+04$ \\
\hline 2 & 23 & $10.150 \mathrm{E}+030.500 \mathrm{E}+04$ \\
\hline 2 & 24 & $10.150 \mathrm{E}+030.500 \mathrm{E}+04$ \\
\hline 2 & 25 & $10.150 \mathrm{E}+03 \quad 0.500 \mathrm{E}+04$ \\
\hline 2 & 26 & $10.150 \mathrm{E}+03 \quad 0.500 \mathrm{E}+04$ \\
\hline 2 & 35 & $10.106 \mathrm{E}+030.500 \mathrm{E}+04$ \\
\hline 2 & 35 & $20.107 E+030.500 E+04$ \\
\hline 2 & 35 & $30.108 E+030.500 E+04$ \\
\hline 2 & 35 & $40.109 \mathrm{E}+030.500 \mathrm{E}+04$ \\
\hline 2 & 35 & $50.110 \mathrm{E}+030.500 \mathrm{E}+04$ \\
\hline 2 & 35 & $60.111 \mathrm{E}+030.500 \mathrm{E}+04$ \\
\hline 2 & 35 & $70.112 E+030.500 E+04$ \\
\hline 2 & 35 & $80.113 E+030.500 E+04$ \\
\hline 2 & 35 & $90.114 \mathrm{E}+030.500 \mathrm{E}+04$ \\
\hline
\end{tabular}

A Ground Water Flow Model for the A/M Area of the SRS (U) 


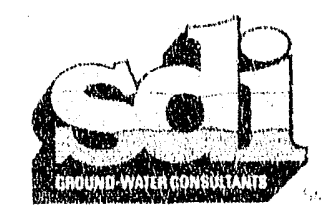

$\begin{array}{llll}2 & 34 & 100.115 \mathrm{E}+030.500 \mathrm{E}+04 \\ 2 & 34 & 110.116 \mathrm{E}+030.500 \mathrm{E}+04 \\ 2 & 33 & 130.119 \mathrm{E}+030.500 \mathrm{E}+04 \\ 2 & 32 & 140.121 \mathrm{E}+030.500 \mathrm{E}+04 \\ 2 & 34 & 120.117 \mathrm{E}+030.500 \mathrm{E}+04 \\ 2 & 33 & 120.118 \mathrm{E}+030.500 \mathrm{E}+04 \\ 2 & 32 & 150.122 \mathrm{E}+030.500 \mathrm{E}+04 \\ 2 & 32 & 160.125 \mathrm{E}+030.500 \mathrm{E}+04 \\ 2 & 31 & 160.127 \mathrm{E}+030.100 \mathrm{E}+05 \\ 2 & 30 & 160.133 \mathrm{E}+030.100 \mathrm{E}+05 \\ 2 & 29 & 160.138 \mathrm{E}+030.100 \mathrm{E}+0.5 \\ 2 & 28 & 160.145 \mathrm{E}+030.100 \mathrm{E}+05 \\ 2 & 27 & 160.150 \mathrm{E}+030.100 \mathrm{E}+05 \\ 2 & 26 & 160.152 \mathrm{E}+030.100 \mathrm{E}+05 \\ 2 & 25 & 160.154 \mathrm{E}+030.100 \mathrm{E}+05 \\ 2 & 24 & 160.156 \mathrm{E}+030.100 \mathrm{E}+05 \\ 2 & 23 & 160.158 \mathrm{E}+030.100 \mathrm{E}+05 \\ 2 & 23 & 170.160 \mathrm{E}+030.100 \mathrm{E}+05 \\ 2 & 27 & 220.146 \mathrm{E}+030.500 \mathrm{E}+04 \\ 2 & 26 & 220.150 \mathrm{E}+030.500 \mathrm{E}+04 \\ 2 & 25 & 220.155 \mathrm{E}+030.500 \mathrm{E}+04 \\ 2 & 24 & 230.160 \mathrm{E}+030.500 \mathrm{E}+04 \\ 2 & 23 & 240.167 \mathrm{E}+030.500 \mathrm{E}+04 \\ 2 & 31 & 170.127 \mathrm{E}+030.500 \mathrm{E}+04 \\ 2 & 30 & 180.128 \mathrm{E}+030.500 \mathrm{E}+04 \\ 2 & 29 & 190.131 \mathrm{E}+030.500 \mathrm{E}+04 \\ 2 & 29 & 200.132 \mathrm{E}+030.500 \mathrm{E}+04 \\ 2 & 29 & 220.135 \mathrm{E}+030.500 \mathrm{E}+04 \\ 2 & 29 & 230.136 \mathrm{E}+030.500 \mathrm{E}+04 \\ 2 & 29 & 240.137 \mathrm{E}+030.500 \mathrm{E}+04 \\ 2 & 30 & 240.138 \mathrm{E}+030.500 \mathrm{E}+04 \\ 2 & 30 & 250.140 \mathrm{E}+030.500 \mathrm{E}+04 \\ 2 & 34 & 90.115 \mathrm{E}+030.500 \mathrm{E}+04 \\ 2 & 29 & 210.133 \mathrm{E}+030.500 \mathrm{E}+04 \\ 2 & 28 & 220.140 \mathrm{E}+030.500 \mathrm{E}+04 \\ 2 & 32 & 130.120 \mathrm{E}+030.500 \mathrm{E}+04 \\ 2 & 29 & 180.130 \mathrm{E}+030.500 \mathrm{E}+04 \\ 1 & 13 & 210.214 \mathrm{E}+030.100 \mathrm{E}+05 \\ 1 & 12 & 210.215 \mathrm{E}+030.100 \mathrm{E}+05 \\ 1 & 12 & 220.215 \mathrm{E}+030.100 \mathrm{E}+05 \\ & & & \end{array}$


APPENDIX 6.5

Output Control Package 
WSSC.OPC

$\begin{array}{rrrr}-1 & 0 & 0 & 0 \\ 0 & 1 & 1 & 1 \\ 1 & 0 & 0 & 0\end{array}$


APPENDIX C6

Recharge Package 
WSSC.RCH

$\begin{array}{ll}3 & 0 \\ 0 & 0\end{array}$

$180.100 \mathrm{E}+01(7 \mathrm{G} 11.4)$

12

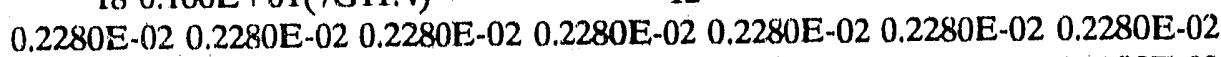

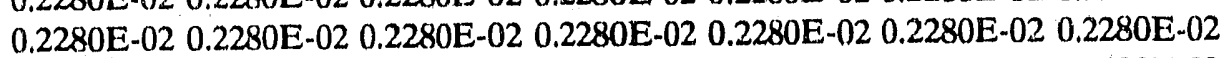
$0.2280 \mathrm{E}-02 \quad 0.2280 \mathrm{E}-02 \quad 0.3420 \mathrm{E}-02 \quad 0.3420 \mathrm{E}-02 \quad 0.3420 \mathrm{E}-02 \quad 0.3420 \mathrm{E}-02 \quad 0.3420 \mathrm{E}-02$ $0.3420 \mathrm{E}-02 \quad 0.3420 \mathrm{E}-02 \quad 0.3420 \mathrm{E}-02 \quad 0.3420 \mathrm{E}-02$

$0.2280 \mathrm{E}-02 \quad 0.2280 \mathrm{E}-02 \quad 0.2280 \mathrm{E}-02 \quad 0.2280 \mathrm{E}-02 \quad 0.2280 \mathrm{E}-02 \quad 0.2230 \mathrm{E}-02 \quad 0.2280 \mathrm{E}-02$ $0.2280 \mathrm{E}-02,0.2280 \mathrm{E}-02 \quad 0.2280 \mathrm{E}-02 \quad 0.2280 \mathrm{E}-02 \quad 0.2280 \mathrm{E}-02 \quad 0.2280 \mathrm{E}-02 \quad 0.2280 \mathrm{E}-02$

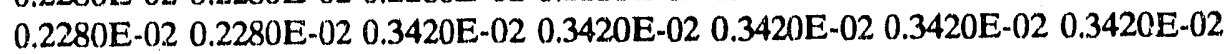
$0.3420 \mathrm{E}-02 \quad 0.3420 \mathrm{E}-02 \quad 0.3420 \mathrm{E}-02 \quad 0.3420 \mathrm{E}-02$

$0.2280 \mathrm{E}-02 \quad 0.2280 \mathrm{E}-02 \quad 0.2280 \mathrm{E}-02 \quad 0.2280 \mathrm{E}-02 \quad 0.2280 \mathrm{E}-02 \quad 0.7280 \mathrm{E}-02 \quad 0.2280 \mathrm{E}-02$ $0.2280 \mathrm{E}-02 \quad 0.2280 \mathrm{E}-02 \quad 0.2280 \mathrm{E}-02 \quad 0.2280 \mathrm{E}-02 \quad 0.2280 \mathrm{E}-02 \quad 0.2280 \mathrm{E}-02 \quad 0.2280 \mathrm{E}-02$ $0.2280 \mathrm{E}-02 \quad 0.2280 \mathrm{E}-02 \quad 0.3420 \mathrm{E}-02 \quad 0.3420 \mathrm{E}-02 \quad 0.3420 \mathrm{E}-02 \quad 0.3420 \mathrm{E}-02 \quad 0.3420 \mathrm{E}-02$ $0.3420 \mathrm{E}-02 \quad 0.3420 \mathrm{E}-02 \quad 0.3420 \mathrm{E}-02 \quad 0.3420 \mathrm{E}-02$

$0.2280 \mathrm{E}-02 \quad 0.2280 \mathrm{E}-02 \quad 0.2280 \mathrm{E}-02 \quad 0.2280 \mathrm{E}-02 \quad 0.2280 \mathrm{E}-02 \quad 0.3420 \mathrm{E}-02 \quad 0.3420 \mathrm{E}-02$ $0.3420 \mathrm{E}-02 \quad 0.3420 \mathrm{E}-02 \quad 0.3420 \mathrm{E}-02 \quad 0.3420 \mathrm{E}-02 \quad 0.3420 \mathrm{E}-02 \quad 0.3420 \mathrm{E}-02 \quad 0.3420 \mathrm{E}-02$ $0.3420 \mathrm{E}-02 \quad 0.3420 \mathrm{E}-02 \quad 0.3420 \mathrm{E}-02 \quad 0.3420 \mathrm{E}-02 \quad 0.3420 \mathrm{E}-02 \quad 0.3420 \mathrm{E}-02 \quad 0.3420 \mathrm{E}-02$ $0.3420 \mathrm{E}-02 \quad 0.3420 \mathrm{E}-02 \quad 0.3420 \mathrm{E}-02 \quad 0.3420 \mathrm{E}-02$

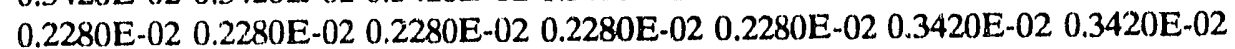
$0.3420 \mathrm{E}-02 \quad 0.3420 \mathrm{E}-02 \quad 0.3420 \mathrm{E}-02 \quad 0.3420 \mathrm{E}-02 \quad 0.3420 \mathrm{E}-02 \quad 6.3420 \mathrm{E}-02 \quad 0.3420 \mathrm{E}-02$ $0.3420 \mathrm{E}-02 \quad 0.3420 \mathrm{E}-02 \quad 0.3420 \mathrm{E}-02 \quad 0.3420 \mathrm{E}-02 \quad 0.3420 \mathrm{E}-02 \quad 0.3420 \mathrm{E}-02 \quad 0.3420 \mathrm{E}-02$ $0.3420 \mathrm{E}-02 \quad 0.3420 \mathrm{E}-02 \quad 0.3420 \mathrm{E}-02 \quad 0.3420 \mathrm{E}-02$

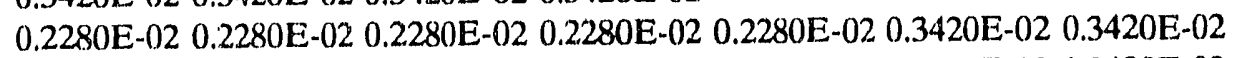
$0.3420 \mathrm{E}-02 \quad 0.3420 \mathrm{E}-02 \quad 0.3420 \mathrm{E}-02 \quad 0.3420 \mathrm{E}-02 \quad 0.3420 \mathrm{E}-02 \quad 0.3420 \mathrm{E}-02 \quad 0.3420 \mathrm{E}-02$ $0.3420 \mathrm{E}-02 \quad 0.3420 \mathrm{E}-02 \quad 0.3420 \mathrm{E}-02 \quad 0.3420 \mathrm{E}-02 \quad 0.3420 \mathrm{E}-02 \quad 0.3420 \mathrm{E}-02 \quad 0.3420 \mathrm{E}-02$ $0.3420 \mathrm{E}-02 \quad 0.3420 \mathrm{E}-02 \quad 0.3420 \mathrm{E}-02 \quad 0.3420 \mathrm{E}-02$

$0.2280 \mathrm{E}-02 \quad 0.2280 \mathrm{E}-02 \quad 0.2280 \mathrm{E}-02 \quad 0.2280 \mathrm{E}-02 \quad 0.2280 \mathrm{E}-02,0.3420 \mathrm{E}-02 \quad 0.3420 \mathrm{E}-02$

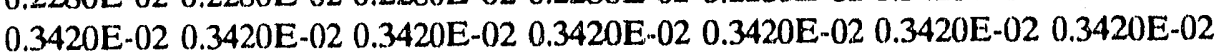
$0.3420 \mathrm{E}-02 \quad 0.3420 \mathrm{E}-02 \quad 0.3420 \mathrm{E}-02 \quad 0.3420 \mathrm{E}-02 \quad 0.3420 \mathrm{E}-02 \quad 0.3420 \mathrm{E}-02 \quad 0.3420 \mathrm{E}-02$ $0.3420 \mathrm{E}-02 \quad 0.3420 \mathrm{E}-02 \quad 0.3420 \mathrm{E}-02 \quad 0.3420 \mathrm{E}-02$

$0.2280 \mathrm{E}-02 \quad 0.2280 \mathrm{E}-02 \quad 0.2280 \mathrm{E}-02,0.2280 \mathrm{E}-02 \quad 0.2280 \mathrm{E}-02 \quad 0.3420 \mathrm{E}-02 \quad 0.3420 \mathrm{E}-02$ $0.3420 \mathrm{E}-02 \quad 0.3420 \mathrm{E}-02 \quad 0.3420 \mathrm{E}-02 \quad 0.3420 \mathrm{E}-02 \quad 0.3420 \mathrm{E}-02 \quad 0.3420 \mathrm{E}-02 \quad 0.3420 \mathrm{E}-02$ $0.3420 \mathrm{E}-02 \quad 0.3420 \mathrm{E}-02 \quad 0.3420 \mathrm{E}-02 \quad 0.3420 \mathrm{E}-02 \quad 0.3420 \mathrm{E}-02 \quad 0.3420 \mathrm{E}-02 \quad 0.3420 \mathrm{E}-02$ $0.3420 \mathrm{E}-02 \quad 0.3420 \mathrm{E}-02 \quad 0.3420 \mathrm{E}-02 \quad 0.3420 \mathrm{E}-02$

$0.2280 \mathrm{E}-02 \quad 0.2280 \mathrm{E}-02 \quad 0.2280 \mathrm{E}-02 \quad 0.2280 \mathrm{E}-02 \quad 0.2280 \mathrm{E}-02 \quad 0.3420 \mathrm{E}-02 \quad 0.3420 \mathrm{E}-02$ $0.3420 \mathrm{E}-02 \quad 0.3420 \mathrm{E}-02 \quad 0.3420 \mathrm{E}-02 \quad 0.3420 \mathrm{E}-02 \quad 0.3420 \mathrm{E}-02 \quad 0.3420 \mathrm{E}-02 \quad 0.3420 \mathrm{E}-02$ $0.3420 \mathrm{E}-02 \quad 0.3420 \mathrm{E}-02 \quad 0.3420 \mathrm{E}-02 \quad 0.3420 \mathrm{E}-02 \quad 0.3420 \mathrm{E}-02 \quad 0.3420 \mathrm{E}-02 \quad 0.3420 \mathrm{E}-02$ $0.3420 \mathrm{E}-02 \quad 0.3420 \mathrm{E}-02 \quad 0.3420 \mathrm{E}-02 \quad 0.3420 \mathrm{E}-02$

$0.2280 \mathrm{E}-02 \quad 0.2280 \mathrm{E}-02 \quad 0.2280 \mathrm{E}-02 \quad 0.2280 \mathrm{E}-02 \quad 0.2280 \mathrm{E}-02 \quad 0.3420 \mathrm{E}-02 \quad 0.3420 \mathrm{E}-02$ $0.3420 \mathrm{E}-02 \quad 0.3420 \mathrm{E}-02 \quad 0.3420 \mathrm{E}-02 \quad 0.3420 \mathrm{E}-02 \quad 0.3420 \mathrm{E}-02 \quad 0.3420 \mathrm{E}-02 \quad 0.342 .0 \mathrm{E}-02$ $0.3420 \mathrm{E}-02 \quad 0.3420 \mathrm{E}-02 \quad 0.3420 \mathrm{E}-02 \quad 0.3420 \mathrm{E}-02 \quad 0.3420 \mathrm{E}-02 \quad 0.3420 \mathrm{E}-02 \quad 0.3420 \mathrm{E}-02$ $0.3420 \mathrm{E}-02 \quad 0.3420 \mathrm{E}-02 \quad 0.3420 \mathrm{E}-02 \quad 0.3420 \mathrm{E}-02$

$0.2280 \mathrm{E}-02 \quad 0.2280 \mathrm{E}-02 \quad 0.2280 \mathrm{E}-02 \quad 0.2280 \mathrm{E}-02 \quad 0.2280 \mathrm{E}-02 \quad 0.3420 \mathrm{E}-020.3420 \mathrm{E}-02$

A Ground Water Flow Model for the A/M Area of the SRS (U)

C6-1 


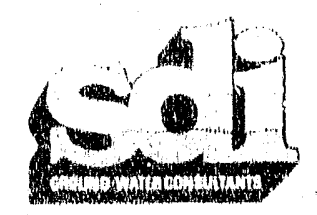

0.3420E-02 0.3420E-02 $0.3420 \mathrm{E}-02 \quad 0.3420 \mathrm{E}-02 \quad 0.3420 \mathrm{E}-02 \quad 0.3420 \mathrm{E}-02 \quad 0.3420 \mathrm{E}-02$ $0.3420 \mathrm{E}-02 \quad 0.3420 \mathrm{E}-02 \quad 0.3420 \mathrm{E}-02 \quad 0.3420 \mathrm{E}-02 \quad 0.3420 \mathrm{E}-02 \quad 0.3420 \mathrm{E}-02 \quad 0.3420 \mathrm{E}-02$ $0.3420 \mathrm{E}-02 \quad 0.3420 \mathrm{E}-02 \quad 0.3420 \mathrm{E}-02 \quad 0.3420 \mathrm{E}-02$

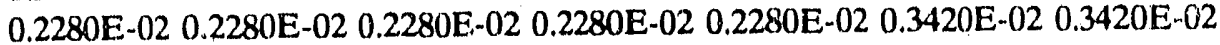
$0.3420 \mathrm{E}-02 \quad 0.3420 \mathrm{E}-02 \quad 0.3420 \mathrm{E}-02 \quad 0.3420 \mathrm{E}-02 \quad 0.3420 \mathrm{E}-02 \quad 0.3420 \mathrm{E}-02 \quad 0.3420 \mathrm{E}-02$

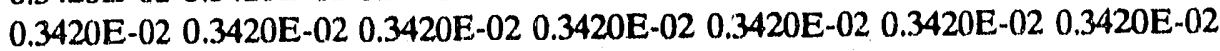
$0.3420 \mathrm{E}-02 \quad 0.3420 \mathrm{E}-02 \quad 0.3420 \mathrm{E}-02 \quad 0.3420 \mathrm{E}-02$

$0.2280 \mathrm{E}-02 \quad 0.2280 \mathrm{E}-02 \quad 0.2280 \mathrm{E}-02 \quad 0.2280 \mathrm{E}-02 \quad 0.2280 \mathrm{E}-02 \quad 0.3420 \mathrm{E}-02 \quad 0.34620 \mathrm{E}-02$

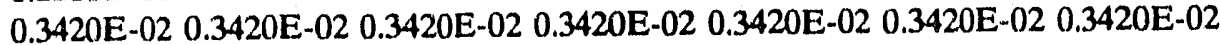
$0.3420 \mathrm{E}-02 \quad 0.3420 \mathrm{E}-02 \quad 0.3420 \mathrm{E}-02 \quad 0.3420 \mathrm{E}-02 \quad 0.3420 \mathrm{E}-02 \quad 0.3420 \mathrm{E}-02 \quad 0.3420 \mathrm{E}-02$ 0.3420E-02 0.3420E-02 0.3420E-02 0.3420E-02

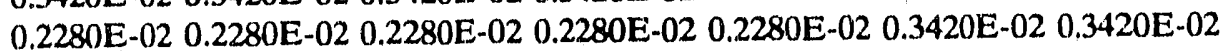

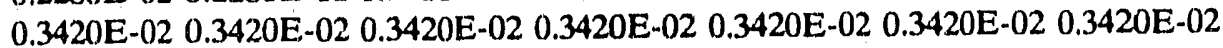
0.3420 E-02 0.3420 E-02 0.3420 E-02 0.3420 E-02 0.3420 E-02 0.3420 E-02 0.3420 E-02 $0.34: 0 \mathrm{E}-02$ 0.3420E-02 0.3420E-02 0.3420E-02

$\therefore .2280 \mathrm{E}-(02) 0.2280 \mathrm{E}-02 \quad 0.2280 \mathrm{E}-02 \quad 0.2280 \mathrm{E}-02 \quad 0.2280 \mathrm{E}-02 \quad 0.3420 \mathrm{E}-02 \quad 0.3420 \mathrm{E}-02$ $0.3420 \mathrm{E}-02 \quad 0.3420 \mathrm{E}-02 \quad 0.3420 \mathrm{E}-02 \quad 0.3420 \mathrm{E}-02 \quad 0.3420 \mathrm{E}-02 \quad 0.3420 \mathrm{E}-02 \quad 0.3420 \mathrm{E}-02$ $0.3420 \mathrm{E}-02 \quad 0.3420 \mathrm{E}-02 \quad 0.3420 \mathrm{E}-02 \quad 0.3420 \mathrm{E}-02 \quad 0.3420 \mathrm{E}-02 \quad 0.3420 \mathrm{E}-02 \quad 0.3420 \mathrm{E}-02$ $0.3420 \mathrm{E}-02$ 0.3420E-02 0.3420E-02 0.3420E-02

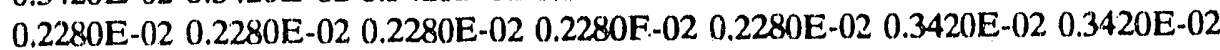
$0.3420 \mathrm{E}-02 \quad 0.3420 \mathrm{E}-02 \quad 0.3420 \mathrm{E}-02 \quad 0.3420 \mathrm{E}-02 \quad 0.3420 \mathrm{E}$ - $-02 \quad 0.2280 \mathrm{E}-02 \quad 0.2280 \mathrm{E}-02$

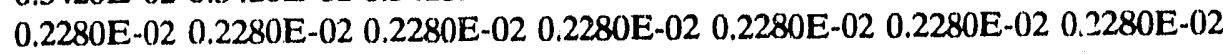

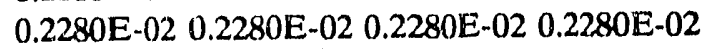

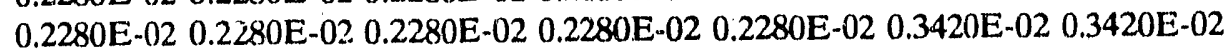
$0.3420 \mathrm{E}-02 \quad 0.3420 \mathrm{E}-02 \quad 0.3420 \mathrm{E}-02 \quad 0.3420 \mathrm{E}-02 \quad 0.3420 \mathrm{E}-02 \quad 0.2280 \mathrm{E}-02 \quad 0.2280 \mathrm{E}-02$

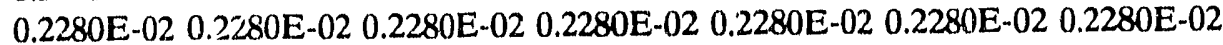
$0.2280 \mathrm{E}-02 \quad 0.2280 \mathrm{E}-02 \quad 0.2280 \mathrm{E}-02,0.2280 \mathrm{E}-02$

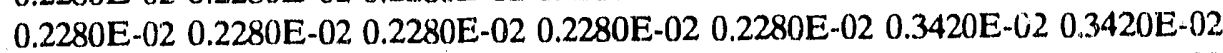

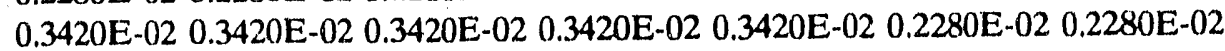

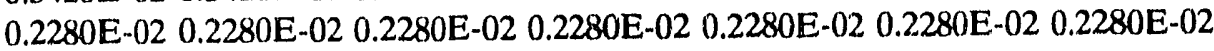
$0.2280 \mathrm{E}-02,0.2280 \mathrm{E}-02 \quad 0.2280 \mathrm{E}-02 \quad 0.2280 \mathrm{E}-02$

$0.2280 \mathrm{E}-02 \quad 0.2280 \mathrm{E}-02 \quad 0.2280 \mathrm{E}-02,0.2280 \mathrm{E}-02 \quad 0.2280 \mathrm{E}-02 \quad 0.3420 \mathrm{E}-02 \quad 0.3420 \mathrm{E}-02$ 0.3420 E-02 0.3420 E-02 0.3420 E-02 0.3420 E-02 0.3420 E-02 0.2280 E-02 $0.2280 \mathrm{E}-02$ $0.2280 \mathrm{E}-02 \quad 0.2280 \mathrm{E}-02 \quad 0.2280 \mathrm{E}-02 \quad 0.2280 \mathrm{E}-02 \quad 0.2280 \mathrm{E}-02 \quad 0.2280 \mathrm{E}-02 \quad 0.2280 \mathrm{E}-02$ $0.2280 \mathrm{E}-02,0.2280 \mathrm{E}-02 \quad 0.2280 \mathrm{E}-02 \quad 0.2280 \mathrm{E}-02$

$0.2280 \mathrm{E}-(12 \quad 0.2280 \mathrm{E}-02 \quad 0.2280 \mathrm{E}-02 \quad 0.2280 \mathrm{E}-02 \quad 0.2280 \mathrm{E}-02 \quad 0.3420 \mathrm{E}-02 \quad 0.3420 \mathrm{E}-02$

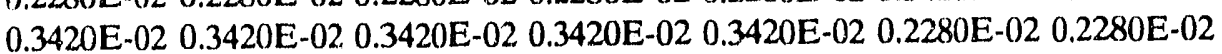
$0.2280 \mathrm{E}-02 \quad 0.2280 \mathrm{E}-02 \quad 0.2280 \mathrm{E}-02 \quad 0.2280 \mathrm{E}-02 \quad 0.2280 \mathrm{E}-02 \quad 0.2280 \mathrm{E}-02 \quad 0.2280 \mathrm{E}-02$ $0.2280 \mathrm{E}-02 \quad 0.2280 \mathrm{E}-02 \quad 0.2280 \mathrm{E}-02 \quad 0.2280 \mathrm{E}-02$

0.2280 E-02 0.2280 E-02 0.2280 E-02 0.2280 E-02 0.2280 E-02 0.3420 E-02 0.3420 E-02 $0.3420 \mathrm{E}-02 \quad 0.3420 \mathrm{E}-02 \quad 0.3420 \mathrm{E}-02 \quad 0.3420 \mathrm{E}-02 \quad 0.3420 \mathrm{E}-02 \quad 0.2280 \mathrm{E}-02 \quad 0.2280 \mathrm{E}-02$

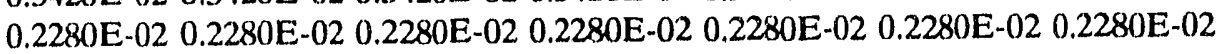
$0.2280 \mathrm{E}-02 \quad 0.2280 \mathrm{E}-02 \quad 0.2280 \mathrm{E}-02,0.2280 \mathrm{E}-02$

$0.2280 \mathrm{E}-02 \quad 0.2280 \mathrm{E}-02 \quad 0.2280 \mathrm{E}-02 \quad 0.2280 \mathrm{E}-02 \quad 0.2280 \mathrm{E}-02 \quad 0.3420 \mathrm{E}-02 \quad 0.3420 \mathrm{E}-02$ 0.3420 E-02 0.3420 E-02 0.3420 E-02 0.3420 E-02 0.3420 E-02 0.2280 E-02 $0.2280 \mathrm{E}-02$ $0.2280 \mathrm{E}-02 \quad 0.2280 \mathrm{E}-02 \quad 0.2280 \mathrm{E}-02 \quad 0.2280 \mathrm{E}-02 \quad 0.2280 \mathrm{E}-02 \quad 0.2280 \mathrm{E}-02 \quad 0.2280 \mathrm{E}-02$ $0.2280 \mathrm{E}-02 \quad 0.2280 \mathrm{E}-02 \quad 0.2280 \mathrm{E}-020.2280 \mathrm{E}-02$

A Ground Water Flow Model for the A/M Area of the SRS (U)

C6-2 


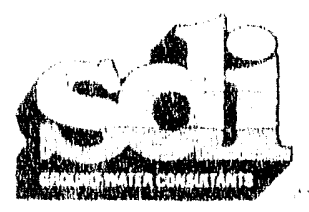

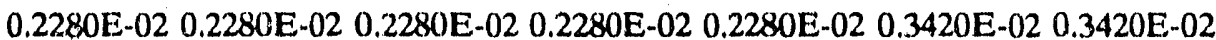
$0.3420 \mathrm{E}-02 \quad 0.3420 \mathrm{E}-02 \quad 0.3420 \mathrm{E}-02 \quad 0.3420 \mathrm{E}-02 \quad 0.3420 \mathrm{E}-02 \quad 0.2280 \mathrm{E}-02 \quad 0.2280 \mathrm{E}-02$ $0.2280 \mathrm{E}-02 \quad 0.2280 \mathrm{E}-02 \quad 0.2280 \mathrm{E}-02 \quad 0.2280 \mathrm{E}-02 \quad 0.2280 \mathrm{E}-02 \quad 0.2280 \mathrm{E}-02 \quad 0.2280 \mathrm{E}-02$ $0.2280 \mathrm{E}-02 \quad 0.2280 \mathrm{E}-02 \quad 0.2280 \mathrm{E}-02,0.2280 \mathrm{E}-02$

$0.2280 \mathrm{E}-02 \quad 0.2280 \mathrm{E}-02 \quad 0.2280 \mathrm{E}-02 \quad 0.2280 \mathrm{E}-02 \quad 0.2280 \mathrm{E}-02 \quad 0.3420 \mathrm{E}-02,0.3420 \mathrm{E}-02$ $0.3420 \mathrm{E}-02 \quad 0.3420 \mathrm{E}-02 \quad 0.3420 \mathrm{E}-02 \quad 0.3420 \mathrm{E}-02 \quad 0.3420 \mathrm{E}-02 \quad 0.2280 \mathrm{E}-02 \quad 0.2280 \mathrm{E}-02$

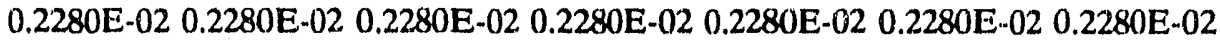
$0.2280 \mathrm{E}-02 \quad 0.2280 \mathrm{E}-02 \quad 0.2280 \mathrm{E}-02 \quad 0.2280 \mathrm{E}-02$


$0.3420 \mathrm{E}-02 \quad 0.3420 \mathrm{E}-02 \quad 0.3420 \mathrm{E}-02 \quad 0.3420 \mathrm{E}-02 \quad 0.3420 \mathrm{E}-02 \quad 0.2280 \mathrm{E}-02.3 .2280 \mathrm{E}-02$ $0.2280 \mathrm{E}-02 \quad 0.2280 \mathrm{E}-02 \quad 0.2280 \mathrm{E}-02 \quad 0.2280 \mathrm{E}-02 \quad 0.2280 \mathrm{E}-02 \quad 0.2280 \mathrm{E}-02 \quad 0.2280 \mathrm{E}-02$ $0.2280 \mathrm{E}-02 \quad 0.2280 \mathrm{E}-02 \quad 0.2280 \mathrm{E}-02 \quad 0.2280 \mathrm{E}-02$

$0.2280 \mathrm{E}-02 \quad 0.2280 \mathrm{E}-02 \quad 0.2280 \mathrm{E}-02 \quad 0.2280 \mathrm{E}-02 \quad 0.2280 \mathrm{E}-02 \quad 0.3420 \mathrm{E}-02 \quad 0.3420 \mathrm{E}-02$ $0.3420 \mathrm{E}-02 \quad 0.3420 \mathrm{E}-02 \quad 0.3420 \mathrm{E}-02 \quad 0.3420 \mathrm{E}-02 \quad 0.3420 \mathrm{E}-02 \quad 0.2280 \mathrm{E}-02 \quad 0.2280 \mathrm{E}-02$ $0.2280 \mathrm{E}-02 \quad 0.2280 \mathrm{E}-02 \quad 0.2280 \mathrm{E}-02 \quad 0.2280 \mathrm{E}-02 \quad 0.2280 \mathrm{E}-02 \quad 0.2280 \mathrm{E}-02.0 .2280 \mathrm{E}-02$ $0.2280 \mathrm{E}-02 \quad 0.2280 \mathrm{E}-02 \quad 0.2280 \mathrm{E}-02 \quad 0.2280 \mathrm{E}-02$

$0.2280 \mathrm{E}-02 \quad 0.2280 \mathrm{E}-02 \quad 0.2280 \mathrm{E}-02 \quad 0.2280 \mathrm{E}-02 \quad 0.2280 \mathrm{E}-02 \quad 0.3420 \mathrm{E}-02 \quad 0.3420 \mathrm{E}-02$ $0.3420 \mathrm{E}-02 \quad 0.2280 \mathrm{E}-02 \quad 0.2280 \mathrm{E}-02 \quad 0.2280 \mathrm{E}-02 \quad 0.2280 \mathrm{E}-02 \quad 0.2280 \mathrm{E}-02 \quad 0.2280 \mathrm{E}-02$ $0.2280 \mathrm{E}-02 \quad 0.2280 \mathrm{E}-02 \quad 0.2280 \mathrm{E}-02 \quad 0.2280 \mathrm{E}-02 \quad 0.2280 \mathrm{E}-02 \quad 0.2280 \mathrm{E}-02 \quad 0.2280 \mathrm{E}-02$ $0.2280 \mathrm{E}-02 \quad 0.2280 \mathrm{E}-02 \quad 0.2280 \mathrm{E}-02 \quad 0.2280 \mathrm{E}-02$

$0.2280 \mathrm{E}-02 \quad 0.2280 \mathrm{E}-02 \quad 0.2280 \mathrm{E}-02 \quad 0.2280 \mathrm{E}-02 \quad 0.2280 \mathrm{E}-02 \quad 0.2280 \mathrm{E}-02 \quad 0.2280 \mathrm{E}-02$ $0.2280 \mathrm{E}-02 \quad 0.2280 \mathrm{E}-02 \quad 0.2280 \mathrm{E}-02 \quad 0.2280 \mathrm{E}-02,0.2280 \mathrm{E}-02 \quad 0.2280 \mathrm{E}-02 \quad 0.2280 \mathrm{E}-02$ $0.2280 \mathrm{E}-02 \quad 0.2280 \mathrm{E}-02 \quad 0.2280 \mathrm{E}-02 \quad 0.2280 \mathrm{E}-02 \quad 0.2280 \mathrm{E}-02 \quad 0.2280 \mathrm{E}-02 \quad 0.2280 \mathrm{E}-02$ $0.2280 \mathrm{E}-02 \quad 0.2280 \mathrm{E}-02 \quad 0.2280 \mathrm{E}-02 \quad 0.2280 \mathrm{E}-02$

$0.2280 \mathrm{E}-02 \quad 0.2280 \mathrm{E}-02 \quad 0.2280 \mathrm{E}-02 \quad 0.2280 \mathrm{E}-02 \quad 0.2280 \mathrm{E}-02 \quad 0.2280 \mathrm{E}-02 \quad 0.2280 \mathrm{E}-02$ $0.2280 \mathrm{E}-02 \quad 0.2280 \mathrm{E}-02 \quad 0.2280 \mathrm{E}-02 \quad 0.2280 \mathrm{E}-02 \quad 0.2280 \mathrm{E}-02 \quad 0.2280 \mathrm{E}-02 \quad 0.2280 \mathrm{E}-02$ $0.2280 \mathrm{E}-02 \quad 0.2280 \mathrm{E}-02 \quad 0.2280 \mathrm{E}-02 \quad 0.2280 \mathrm{E}-02 \quad 0.2280 \mathrm{E}-02 \quad 0.2280 \mathrm{E}-02 \quad 0.2280 \mathrm{E}-02$ $0.2280 \mathrm{E}-02 \quad 0.2280 \mathrm{E}-02 \quad 0.2280 \mathrm{E}-02 \quad 0.2280 \mathrm{E}-02$

$0.2280 \mathrm{E}-02 \quad 0.2280 \mathrm{E}-02 \quad 0.2280 \mathrm{E}-02 \quad 0.2280 \mathrm{E}-02 \quad 0.2280 \mathrm{E}-02 \quad 0.2280 \mathrm{E}-02 \quad 0.2280 \mathrm{E}-02$

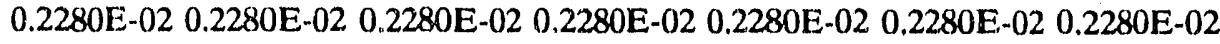
$0.2280 \mathrm{E}-02$
$0.2280 \mathrm{E}-02$ $0.2280 \mathrm{E}-02 \quad 0.2280 \mathrm{E}-02 \quad 0.2280 \mathrm{E}-02 \quad 0.2280 \mathrm{E}-02$

$0.2280 \mathrm{E}-02 \quad 0.2280 \mathrm{E}-02 \quad 0.2280 \mathrm{E}-02 \quad 0.2280 \mathrm{E}-02 \quad 0.2280 \mathrm{E}-02 \quad 0.2280 \mathrm{E}-02 \quad 0.2280 \mathrm{E}-02$

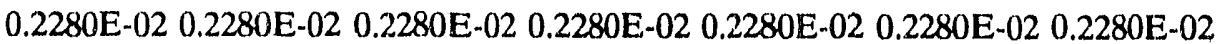
$0.2280 \mathrm{E}-02 \quad 0.2280 \mathrm{E}-02 \quad 0.2280 \mathrm{E}-0.20 .2280 \mathrm{E}-02 \quad 0.2280 \mathrm{E}-02 \quad 0.2280 \mathrm{E}-02 \quad 0.2280 \mathrm{E}-02$ $0.2280 \mathrm{E}-02 \quad 0.2280 \mathrm{E}-02 \quad 0.2280 \mathrm{E}-02 \quad 0.2280 \mathrm{E}-02$

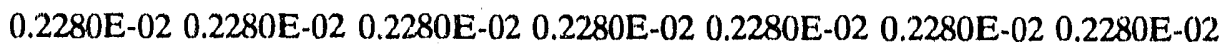
$0.2280 \mathrm{E}-020.2280 \mathrm{E}-02 \quad 0.2280 \mathrm{E}-02 \quad 0.2280 \mathrm{E}-02 \quad 0.2280 \mathrm{E}-02 \quad 0.2280 \mathrm{E}-02.0 .2280 \mathrm{E}-02$ $0.2280 \mathrm{E}-02 \quad 0.2280 \mathrm{E}-02 \quad 0.2280 \mathrm{E}-02 \quad 0.2280 \mathrm{E}-02 \quad 0.2280 \mathrm{E}-02 \quad 0.2280 \mathrm{E}-02 \quad 0.2280 \mathrm{E}-02$ $0.2280 \mathrm{E}-02 \quad 0.2280 \mathrm{E}-02 \quad 0.2280 \mathrm{E}-02 \quad 0.2280 \mathrm{E}-02$

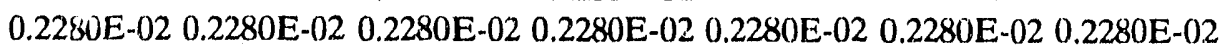

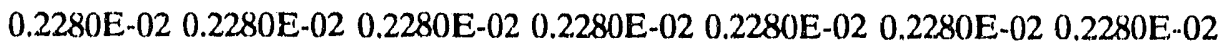
$0.2280 \mathrm{E}-02 \quad 0.2280 \mathrm{E}-02 \quad 0.2280 \mathrm{E}-02 \quad 0.2280 \mathrm{E}-02,0.2280 \mathrm{E}-02 \quad 0.2280 \mathrm{E}-02 \quad 0.2280 \mathrm{E}-02$ $0.2280 \mathrm{E}-02 \quad 0.2280 \mathrm{E}-02 \quad 0.2280 \mathrm{E}-02 \quad 0.2280 \mathrm{E}-02$

$0.2280 \mathrm{E}-02 \quad 0.2280 \mathrm{E}-02 \quad 0.2280 \mathrm{E}-02 \quad 0.2280 \mathrm{E}-02 \quad 0.2280 \mathrm{E}-02 \quad 0.2280 \mathrm{E}-02 \quad 0.2280 \mathrm{E}-02$ $0.2280 \mathrm{E}-02 \quad 0.2280 \mathrm{E}-02 \quad 0.2280 \mathrm{E}-02 \quad 0.2280 \mathrm{E}-02 \quad 0.2280 \mathrm{E}-02 \quad 0.2280 \mathrm{E}-02 \quad 0.2280 \mathrm{E}-02$ $0.2280 \mathrm{E}-02 \quad 0.2280 \mathrm{E}-02 \quad 0.2280 \mathrm{E}-02 \quad 0.2280 \mathrm{E}-02 \quad 0.2280 \mathrm{E}-02 \quad 0.2280 \mathrm{E}-02 \quad 0.2280 \mathrm{E}-02$

A Ground Water Flow Model for the A/M Area of the SRS (U)

C6-3 


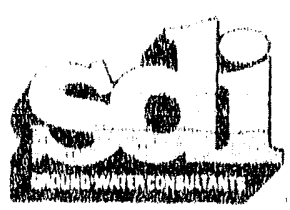

$0.2280 \mathrm{E}-02 \quad 0.2280 \mathrm{E} \quad 02 \quad 0.2280 \mathrm{E}-02 \quad 0.2280 \mathrm{E}-02$

$0.2280 \mathrm{E}-02 \quad 0.2280 \mathrm{E}-02 \quad 0.2280 \mathrm{E}-02 \quad 0.2280 \mathrm{E}-02 \quad 0.2280 \mathrm{E}-02 \quad 0.2280 \mathrm{E}-02 \quad 0.2280 \mathrm{E}-02$

$0.2280 \mathrm{E}-02 \quad 0.2280 \mathrm{E}-02 \quad 0.2280 \mathrm{E}-02 \quad 0.2280 \mathrm{E}-02 \quad 0.2280 \mathrm{E}-02 \quad 0.2280 \mathrm{E}-02 \quad 0.2280 \mathrm{E}-02$

$0.2280 \mathrm{E}-02 \quad 0.2280 \mathrm{E}-02 \quad 0.2280 \mathrm{E}-02 \quad 0.2280 \mathrm{E}-02 \quad 0.2280 \mathrm{E}-02 \quad 0.2280 \mathrm{E}-02 \quad 0.2280 \mathrm{E}-02$

$0.2280 \mathrm{E}-02 \quad 0.2280 \mathrm{E}-02 \quad 0.2280 \mathrm{E}-02 \quad 0.2280 \mathrm{E}-02$

A Ground Water Flow Model for the A/M Area of the SRS (U)

C6-4 


\section{APPENDIX C7}

Sip Solution Package 


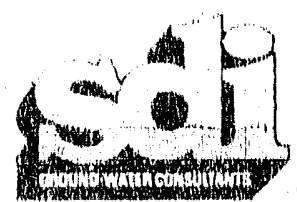

WSSC.SIP

$\begin{array}{cccc}300 & 5 & & \\ .20000 & .10000 \mathrm{E}-04 & 1.00000 \mathrm{E}+\infty & 1\end{array}$




\section{APPENDIX D}

Location and Pumpage Rates of Production and Recovery Wells Used in Groundwater Model 


\section{APPENDIX D}

\section{LOCATION OF WELLS AND PUMPING RATES USED IN GROUNDWATER MODEL}

\begin{tabular}{|c|c|c|c|c|c|c|c|}
\hline \multirow{2}{*}{ Well No. } & \multirow{2}{*}{$\begin{array}{c}\text { North SRP } \\
(\mathrm{ft})\end{array}$} & \multirow{2}{*}{$\begin{array}{l}\text { East SRP } \\
\text { (ft) }\end{array}$} & \multirow{2}{*}{$\begin{array}{c}\text { Total } \\
\text { Pumping } \\
\text { Rate } \\
\text { (gpm) }\end{array}$} & \multicolumn{4}{|c|}{ Pumpage From Model Layer (gpm)* } \\
\hline & & & & 1 & 2 & 3 & 4 \\
\hline PW-53A & 105011 & 50757 & 350 & & & & 116.7 \\
\hline PW-20A & 104000 & 50,615 & 350 & 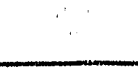 & & & 116.7 \\
\hline$P W-82 A$ & 103330 & 51100 & 400 & & & & 133.3 \\
\hline $\mathrm{PW}-112 \mathrm{G}$ & 99388 & 58023 & 500 & & & & 116.7 \\
\hline$P W-113 G$ & 99961 & 57023 & 500 & & & & 116.7 \\
\hline $\mathrm{PW}-67 \mathrm{~B}$ & 86693 & 42622 & 75 & & & & 2.5 \\
\hline PW-68A & 106266 & 50266 & 30 & & & & 10 \\
\hline RWM-1 & 102608 & 48581 & 27 & 1.4 & 17.1 & 8.5 & \\
\hline RWM-2 & 104434 & 49206 & 24 & 1.2 & 15 & 7.8 & \\
\hline RWM-3 & 104730 & 49680 & 57 & 2.9 & 36.1 & 18 & \\
\hline RWM-4 & 103719 & 48948 & 42 & 2.1 & 26.6 & 13.3 & \\
\hline RWM-5 & 103502 & 49628 & 5 & 0.3 & 3.2 & 1.5 & \\
\hline R.WM-6 & 102002 & 50107 & 50 & 2.5 & 31.7 & 15.8 & \\
\hline RWM-7 & 101905 & 49450 & 29 & 1.5 & 18.4 & 9.1 & \\
\hline RWM-8 & 101948 & 47353 & 34 & 1.7 & 21.5 & 10.8 & \\
\hline RWM-9 & 104100 & 50400 & 34 & 1.7 & 21.5 & 10.8 & \\
\hline RWM-10 & 102001 & 48244 & 60 & 3 & 38 & 19 & \\
\hline RWM-11 & 104875 & 50400 & 65 & 3.3 & 41.2 & 20.5 & \\
\hline
\end{tabular}

*Production wells in layer 4 are also pumping from the Middendorf (lower portion of Aquifer System I) which is not simulated in this model. 


\section{APPENDIX E}

Description of Calibration Process 


\section{APPENDIX E}

\section{Summary of Runs Made During Coarse Grid Model Calibration}

SDI started with the parameters used in the coarse grid model calibrated by S.S. Papadopulos \& Associates (SSP\&A) in 1987. The only deviation from the Papadopulos model was combining the Ellenton and the Lower Congaree layers into one model layer and utilizing a leakance coefficient to simulate the Ellenton as a confining unit below the Upper Congaree. SDI reproduced the predicted water levels reported by Papadopulos (1987).

The 1989-1990 observed water levels differ from the model-predicted water levels produced by the SSP\&A calibration. This may be attributed to the effect of several years of recovery well pumpage and a decline in rainfall rates. Model-predicted water levels were higher than observed water levels. Additional monitoring wells have been installed since the SSP\&A calibration and understanding of the hydrogeologic system has improved. SDI analyzed the available data and recaliorated the model to 1989-1990 obscrved water levels, surface water flow, and aquifer test data. The following text describes the steps taken in the recalibration.

The groundwater flow direction and water levels did not match observed data in layer 4 (Black Creek). A number of runs changing boundary conditions in layer 4 and leakance values in layer 3 were made in an effort to reduce the amount of water entering layer 4 . None of the runs satisfactorily improved the calibration in this layer. Boundary conditions and leakance were returned to the SSP\&A parameters. 
The SSP\&A model had a ring of inactive nodes around each layer. These nodes are not necessary in MODFLOW to simulate boundary conditions. The outer ring of inactive nodes were converted to active nodes in all layers. After reviewing available data, the layer boundaries were further extended in layers 1 through 3 . Layer 1 was extended 2 nodes to the west to incorporate new well data and layer 2 was expanded to the south to match the boundary of layer 3. In the SSP\&A model, layer 3 stopped just north of Upper Three Runs Creek. The expanded layer boundaries improved calibration along the edges of the model in layers 1 through 3.

The well package was updated by including the new pumpage rates for the production and recovery wells provided by SRL. Pumpage rates for the recovery wells were prorated between layers 1,2 and 3 according to the transmissivity of the layers (Appendix D).

A number of runs were devoted to assessing the model's sensitivity to recharge. A recharge rate of $15 \mathrm{in} / \mathrm{yr}$ was used over the entire model area in the SSP\&A calibration. A run using $12 \mathrm{in} / \mathrm{yr}$ and a run using $18 \mathrm{in} / \mathrm{yr}$ were done. The simulation using $12 \mathrm{in} / \mathrm{yr}$ resulted in a good calibration for the southern half of the model, but did not provide enough water in the $\mathrm{A} / \mathrm{M}$ Area. The simulation using $18 \mathrm{in} / \mathrm{yr}$ resulted in a good calibration in the $\mathrm{A} / \mathrm{M}$ Area but too much water in the southern portion of the model. The model was found to be quite sensitive to recharge. Recharge was returned to $15 \mathrm{in} / \mathrm{yr}$.

After these adjustments, water levels in all layers of the model were still too high in the southern two-thirds of the model. The reasons for the high groundwater levels was thought to be due to one or more of three factors: 1) too much recharge; 2) too little downward leakage; and/or 3) transmissivity values that are too low. The analyses A Ground Water Flow Model for the A/M Area of the SRS (U) Page E-2 
showed that the high water levels in layers 2 and 3 caused most of the water entering layer 4 through constant head nodes in the upper part of the modeled area to flow upward into layer 3 along Upper Three Runs Creek.

After reviewing the regional hydrostratigraphic cross-section (hereafter referred to as the regional cross-section) sent by SRL (Figure 6) it was observed that both the Upper and Lower Congaree thicken to the south. A series of model runs were made to investigate the effects of the transmissivity distribution and value on the calibration. The model was found to be less sensitive to changes in transmissivity than recharge and leakance.

As in the SSP\&A model, the total transmissivity for the Upper and Lower Congaree is $2,630 \mathrm{ft}^{2} /$ day in the northern half of the model. A hydraulic conducti $/$ ity of $44 \mathrm{ft} /$ day is used for both layers (Papadopulos, 1987). The thicknesses of the Upper and Lower Congaree in the $\mathrm{A} / \mathrm{M}$ Area are $40 \mathrm{feet}$ and $20 \mathrm{feet}$, respectively. The transmissivity of both the Upper and Lower Congaree was increased to the south to account for the thickening of strata shown in the regional cross-section. These distributions of transmissivity resulted in a marked improvement of the calibration over the entire model.

At this point in the calibration, layer 4 , which had a transmissivity of 12,500 $\mathrm{ft}^{2} / \mathrm{day}$, still had too much water. The total transmissivity for the Black Creek and the Middendorf has been calculated to range from 10,400 to $14,200 \mathrm{ft}^{2} /$ day based on an aquifer pumping test on production well PW-20A. The hydraulic conductivity in the Black Creek is estimated as half of the hydraulic conductivity of the Middendorf. Using a hydraulic conductivity of $23.5 \mathrm{ft} / \mathrm{day}$ for the Black Creek and a thickness of $170 \mathrm{ft}$ from the regional cross-section, the transmissivity of the Black Creek in the SRL 
is approximately $4,000 \mathrm{ft}^{2} / \mathrm{day}$. The value of transmissivity for the Black Creek (layer 4) was reduced from $12,500 \mathrm{ft}^{2} /$ day to $4,000 \mathrm{ft}^{2} /$ day (Figure 18). This change improved the calibration of the water level elevations and gradient in layer 4 and reduced the upward flow to layer 3 beneath Upper Three Runs Creek.

The boundaries of both layers 2 and 3 were extended over the entire model area as a result of information from the regional cross-section and the Duffield et al. (1989) report on the $F$ and $H$ areas. Based on the regional cross-section and conversations with SRL, the Upper and Lower Congaree are modeled as a continuous unit in the southern half of the model. The leakance in layer 2 was raised in the southern half of the model to simulate the absence of a confining unit between the Upper and Lower Congarie.

As in the SSP\&A model, the discharge of groundwater to streams and wetlands was simulated in this model by nodes with head-dependent discharge (general-head boundary nodes). Extending the Upper and Lower Congaree layer boundaries made it necessary to relocate the general-head boundary (GHB) nodes directly over Upper Three Runs Creek. The GHB nodes were previously along the southern boundaries of layers 2 and 3 just north of Upper Three Runs Creek. The regional cross-section as well as cross-sections in Christensen and Gordon (1983) indicate that Upper Three Runs Creek is incised into the Upper Congaree. Therefore, all GHB nodes that were in layer 3 were raised to layer 2 .

The flow through general head boundary nodes is regulated by the assigned head at the node and a conductance term that simulates the hydraulic properties of the stream bottom. Heads in each node were chosen from a topographic map (Department of Energy, 1987). The conductance terms were calculated then adjusted during calibration. The flow into and out of the GHB nodes was calibrated to the stream flow A Ground Water Flow Model for the A/M Area of the SRS (U) Page E-4 
data SDI received for Tirn's Branch and Upper Three Runs Creek. The Lockwood Greene Engineers, Inc. (1983) report on the stream flow of 'Tim's Branch indicates that the upper portion of Tim's Branch has only seasonal flow. Because this portion of the stream is not a constant source of water, some of the GHB nodes were eliminated from the upper reach of Tim's Branch in layer 1 (Figure 15). This improved the calibration in the water table.

As mentioned previously, the model was found to be sensitive to recharge rates. Quite a few runs were made in an effort to use one recharge rate and balance the deficiency of water in the north with the abundance of water in the south using leakance, transmissivity and GHB nodes. These simulations were not able to match the observed water levels without using unrealistic values for one or more parameters. A range in recharge rates of 8 to $15 \mathrm{in} / \mathrm{yr}$ have been used in past models in the area. To achieve the desired water levels in the southern half of the model recharge was cut from $15 \mathrm{in} / \mathrm{yr}$ to $10 \mathrm{in} / \mathrm{yr}$. The resulting water levels were too low in the northeast portion of the model. More water was needed in the $\mathrm{A} / \mathrm{M}$ Area and along the ridge between Tim's Branch and the southwest wetlands. Recharge was increased to $15 \mathrm{in} / \mathrm{yr}$ over the top of the ridge and left at $10 \mathrm{in} / \mathrm{yr}$ over the areas of steeper topography. Figure 22 illustrates the final recharge distribution for the entire model area. After this adjustment, the only area remaining deficient in water level elevation was the $A / M$ Area in layers 1 and 2.

The variable recharge distribution resulted in the correct range of water levels in each layer. However, the flow directions and gradients did not match the observed water level contours in all areas of the model. It was found that boundary conditions needed to be changed in some places to simulate the flow indicated by the observed 
water levels. Constant-heads were added to the north and west sides of layer 1 to simulate flow from the ridge toward areas of lower elevation. Constant-heads were also added to layer 2 and layer 3 , where observed water levels also suggest flow out of the northeast corner. The final boundary conditions used in this calibration are in Figure 15 through 18.

To further improve the calibration of the coarse grid model calibration, leakance values in each layer were adjusted. Some of the final leakance zones configurations are similar to those in the SSP\&A calibration. The model is sensitive to leakance rates in the A/M Area where hydraulic head differences between the layers are the largest. A series of runs were necessary to balance leakance rates between the layers and to adjust the leakance zones to match the observed water level contours in each layer.

In layer 1, a slightly lower leakance was necessary in the $A / M$ Area to match the observed water levels (Figure 19). The leakance value in layer 2 is determined by the thickness of the clay layer between the Upper and Lower Congaree. The clay appears to be the thickest in the $A / M$ Area and thins and becomes discontinuous to the south. Leakance is highest in the southern sector where the Congaree is thinnest and data points from Fallaw and Sargent (1986) show the clay layer to be absent (Figure 20). Leakance in layer 3 is determined by the thickness of the clays in the Ellenton (Figure 21). Data from Fallaw and Sargent (1986) indicates that the clay is thickest in the A/M Area and to the southeast beneath Tim's Branch.

At this point the coarse grid model was calibrated and sent to SRL for review. SDI was advised of a specific capacity test run on a new recovery well, $R W-16$, in the southern sector. The specific capacity of $4 \mathrm{gpm} / \mathrm{ft}$ suggested a lower transmissivity in the Upper and Lower Congaree than was modeled. Further investigation revealed that A Ground Water Flow Model for the A/M Area of the SRS (U) Page E-6 
the Upper and Lower Congaree may be thinner southeast of the A/M Area (Fallaw, 1990). A block of lower transmissivity was added to layers 2 and 3. The shape of the block was determined by isopach data of the Upper and Lower Congaree in Fallaw (1990). The final distribution of transmissivity in the Upper Congaree is shown in Figure 16. The final distribution of transmissivity in the Lower Congaree is shown in Figure 17. Leakance rates in layer 2 and layer 3 were adjusted to recalibrate to the observed water levels. 

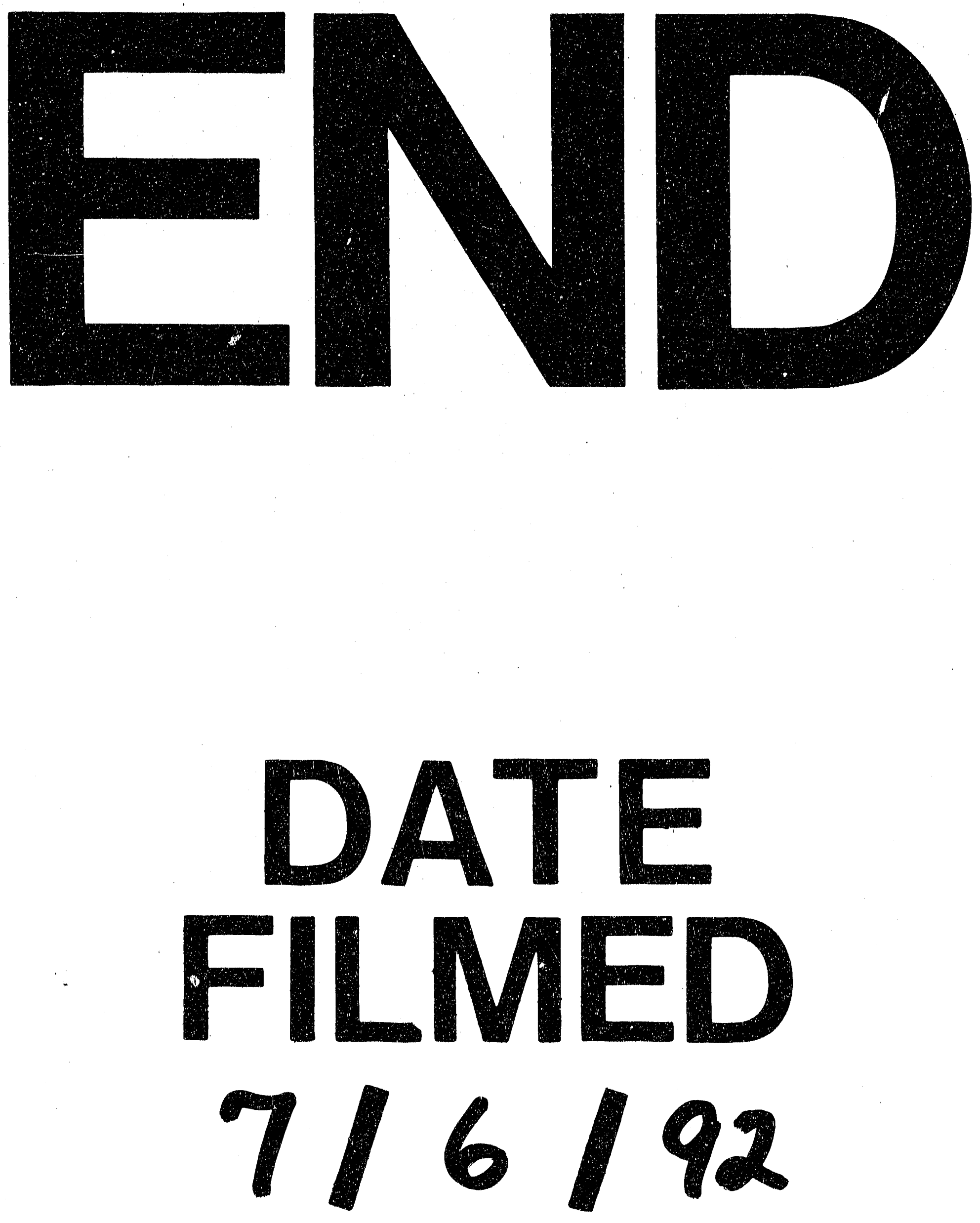
